RECEIVED

Y/ER/SUB/95-005MS

DEC 191996

OST

\title{
Post-Closure Permit Application \\ for the \\ Kerr Hollow Quarry at the Y-12 Plant
}

\section{June 1995}

Prepared for

LOCKHEED MARTIN ENERGY SYSTEMS, INC.

Oak Ridge, Tennessee

DISTRBUTTOON OF THIS DOCUMENT IS UNLMMTED

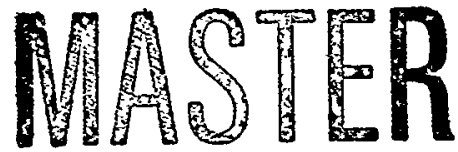

Prepared by

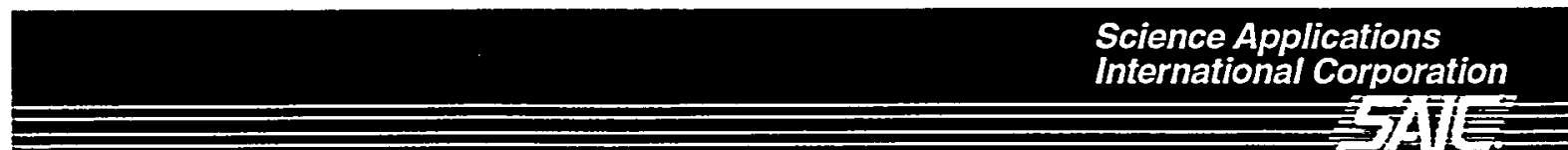

P.O. Box 2502

800 Oak Ridge Turnpike

Oak Ridge, Tennessee 37831 
DISCLAIMER

Portions of this document may be illegible in electronic image products. Images are produced from the best available original document. 
on three sides by 60 -foot high cliffs. Access to the quarry is limited by a 7-foot high, chain-link, barbed-wire-topped fence. The gates of the fence are locked at all times except for authorized entry.

Beginning in the early 1950s the quarry was used for waste disposal of a variety of materials, principally water-reactive materials but also shock-sensitive chemicals and compressed gas cylinders. To facilitate this use of the quarry, a metal building and chute were constructed on the southern bluff of the quarry, high above the water surface. Water-reactive materials such as lithium, potassium, calcium, sodium, and sodium-potassium alloy that were to be disposed of were packaged in 5-, 30-, or 55-gallon containers and transported to the quarry (DOE 1993). There the containers were placed in the chute and dropped to the water's surface. In transit to the water's surface, the containers were punctured by rifle fire from Y-12 Plant security guards, allowing the contents of the containers to react with the quarry water. Explosions and violent burning upon impact with the water's surface, or shortly after sinking, indicated that the chemicals inside the containers reacted completely. A limited number of 500-gallon pressure vessels containing sodium was also placed in the quarry. These containers were dropped off the southern bluff and were allowed to vent by opening valves and vent pipes. All of these containers sank as a result of their great weight.

Potentially explosive chemicals, such as picric acid, were transported to the quarry in specially packed containers to minimize shock and possible detonation in transit. The containers were suspended on a cable above the water surface and punctured with rifle fire prior to being released into the water (DOE 1993).

In addition to disposal of water-reactive and explosive chemicals, the quarry was also used to vent gas cylinders with frozen valves. These were placed on the northwest bank of the quarry and punctured with rifle fire. Vented, empty cylinders were typically returned to the main Y-12 Plant for disposal; however, prior to 1970, cylinders were discarded in the quarry (DOE 1993).

Disposal operations at KHQ were terminated on November 7, 1988 (DOE 1993). Termination was in response to an October 11, 1987 determination by the Tennessee Department of Environment and Conservation (TDEC) under the Tennessee Water Quality Control Act that KHQ was a surface impoundment subject to the 1984 Hazardous and Solid Waste Amendments 


\title{
POST-CLOSURE PERMIT APRLICATION \\ FOR THE KERR HOLLOW QUARRY \\ AT THE Y-12 PLANT
}

\author{
Document:Prepared by \\ SCIENCE APPLICATIONS RNTERNATIONAL CORPORATION \\ - $\mathrm{P}_{\mathrm{i}} \mathrm{O}$. Box 2502 \\ 800 Oak Ridge Turnpike \\ Oak Ridge, Tennessee $\mathbf{3 7 8 3 1}$
}

for

U.S. Department of Energy

Office of Environmental Restoration and Waste Management under budget and reporting code EW20

Oak Ridge Y-12 Plant

Oak Ridge, Tennessee 37831

managed by

LOCKHEED MARTIN ENERGY SYSTEMS, INC.

for the U.S. DEPARTMENT OF ENERGY

Under Contract No. DE-AC05-84OR21400 
(HSWA) of the Resource Conservation and Recovery Act (RCRA) (DOE 1993). Under such a determination, the quarry had to satisfy technical requirements of HSWA (i.e., lining) or be closed. Because lining the quarry was impracticable, methods of closure were reviewed. A closure plan was developed for $\mathrm{KHQ}$, submitted to TDEC, and conditionally approved on September 28, 1988 (Fraser et al. 1993). Several revisions of the original closure plan were drafted to account for unanticipated conditions. Most notably, the approved D2 revision of the KHQ Closure Plan identified partial and final closure requirements and changed the KHQ closure from "clean" to "dirty." This change was to allow the sediment and any objects buried by the sediment to remain within the quarry (DOE 1993).

Upon termination of disposal activities, certain actions were initiated to close the quarry in accordance with the closure requirements for interim status surface impoundments specified in Tennessee Rules 1200-1-11-.05(7) and 1200-1-11-.05(11). As part of these actions, efforts were undertaken to characterize the physical and chemical nature of wastes that had been disposed of in the quarry, and to remove any containers or debris that were put in the quarry during waste disposal activities. Closure certification reports (Fraser et al. 1993 and Dames and Moore 1993) document closure activities in detail; a summary is presented in the following paragraphs.

An underwater remote-operated vehicle (ROV) equipped with a video camera was used to survey the bottom of the quarry from February to March 1989. The survey showed that most of the 5-, 30-, and 55-gallon containers visible to the camera appeared to be empty of any residual reactive or explosive materials. Additional surveys were made during the spring and early summer of 1991 to locate the large steel vessels that had also been disposed of in the quarry. By virtue of their design and the method for disposal of these vessels, it was impossible to determine whether their contents had fully reacted with the quarry water. This necessitated using a remote method to confirm that they no longer contained reactive chemicals. The method consisted of detonating explosive charges on the side of the vessel to ensure venting, then viewing the ruptured vessel with a remote-controlled camera aboard an ROV.

Removal of items from the quarry was accomplished using an ROV and a remote-operated barge equipped with a grapple. The ROV was used mainly for small items and the barge and grapple for large items. Except for the large steel vessels, both retrieval systems picked up and placed items into a shredder on the quarry bottom. Shredded debris were collected in a basket 
on the bottom of the shredder and the basket was removed when it became full. The debris were then inspected and stored in an area located near the quarry. The explosively breached large steel vessels were removed from the quarry without being shredded using the barge and grapple.

The ROV also identified compressed gas cylinders at the bottom of the quarry. For their removal, the grapple on the barge was replaced with a cylinder breaching grapple. This device had the capability of grasping the cylinders and puncturing the cylinders with a hydraulic punch. Initially, it was assumed that all compressed gas cylinders had been vented prior to disposal in the quarry and breaching was done nonetheless as a precaution. In March 1992, however, pressurized gas cylinders were encountered and breached.

Other containers (e.g., 5-, 30-, and 55-gallon containers) that were not obviously empty were shredded underwater. This process exposed any reactive materials that remained in the containers to water. The shredded material was removed from the quarry. Obviously empty items were inspected and then removed from the quarry as nonhazardous waste.

The final closure action was performed by dividing the quarry bottom into 30 -foot square sectors, and mapping and labeling the remaining contents. All visible containers or structures (with two exceptions trapped by rock slides) were removed from the quarry via remote operation. The term "visible" was defined as 3 feet above the bottom of the quarry due to the operational limits of the ROV, and "containers or structures" were defined as any item that might contain reactive material that had not been exposed to or reacted with the quarry water. A final inspection was documented with 80 hours of video tape in October 1993, and the operating equipment was removed from the quarry on November 11, 1993. Seventy-three visible items that met the definition of nonhazardous to human health and the environment were allowed to remain in the quarry. 


\section{POST-CLOSURE PERMIT APRLICATION \\ FOR THE KERR HOLLOW QUARRY \\ AT THE Y-12 PLANT}

Document: Prepared by

\section{SCIENCE APPLICATIONS INTERNATIONAL CORPORATION}

Po. Box 2502

800 Oalk Ridge Turnpike

Oak Ridge, Tennessee 37831

\section{for}

\section{U.S. Department of Energy}

Office of Environmental Restoration and Waste Management under budget and reporting code EW20

Oak Ridge Y-12 Plant

Oak Ridge, Tennessee 37831 managed by

LOCKHEED MARTIN ENERGY SYSTEMS, INC. for the U.S. DEPARTMENT OF ENERGY Under Contract No. DE-AC05-84OR21400 


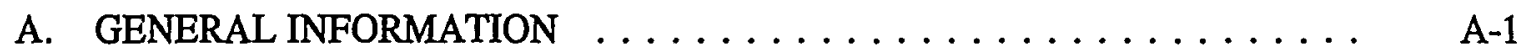

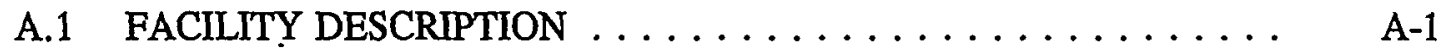

A.2 WASTE CHARACTERISTICS ................. A

A.3 FACILITY LOCATION $\ldots \ldots \ldots \ldots \ldots \ldots$ A 6

A.4 TRAFFIC PATTERNS $\ldots \ldots \ldots \ldots \ldots \ldots \ldots \ldots$ A $6 \ldots \ldots$

A.5 SECURITY PROCEDURES AND EQUIPMENT . . . . . . . . A A

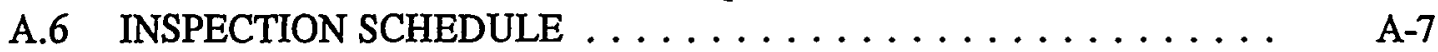

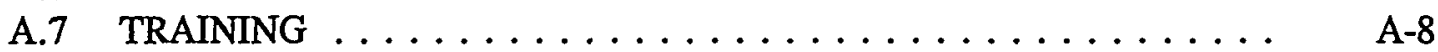

A.8 CLOSURE PLAN AND POST-CLOSURE ACTIVITIES . . . . . . . A-8

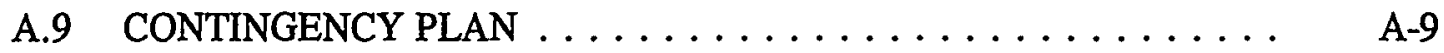

A.10 NOTICES REQUIRED FOR DISPOSAL FACILITIES . . . . . . . . A A 9

A.11 CLOSURE AND POST-CLOSURE COST ESTIMATES . . . . . . . . . A A

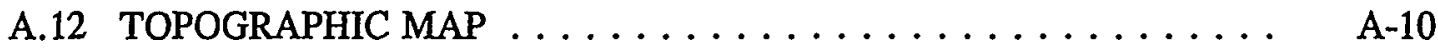

A.13 LIABILITY INSURANCE . . . . . . . . . . . . . . A A-10

A.14 POST-CLOSURE CONTACT $\ldots \ldots \ldots \ldots \ldots \ldots \ldots$ A-10

B. GROUNDWATER INFORMATION . . . . . . . . . . . . . . . B B

B.1 SUMMARY OF INTERIM STATUS DATA . . . . . . . . . . . B-1

B.1.1 Monitoring Well Network ................ B-1

B.1.2 Groundwater Sampling and Analysis . . . . . . . . . . . . . . . .

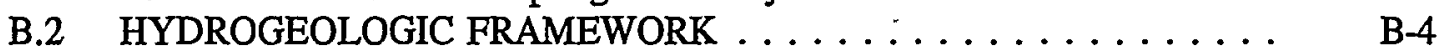

B.2.1 Regional Geology $\ldots \ldots \ldots \ldots \ldots \ldots \ldots \ldots \ldots \ldots$ B-4

B.2.2 Stratigraphic Units $\ldots \ldots \ldots \ldots \ldots \ldots \ldots \ldots \ldots$ B-5

B.2.2.1 Unconsolidated deposits .............. B-5

B.2.2.2 Bedrock geology ................ B . .

B.2.3 Structural Relationships . . . . . . . . . . . . . B-6

B.2.4 Surface Water System . . . . . . . . . . . . . . B-7

B.2.4.1 Southeast Chestnut Ridge Watershed ......... B-7

B.2.4.2 Surface water use $\ldots \ldots \ldots \ldots \ldots \ldots \ldots$ B-7

B.2.4.3 Precipitation data $\ldots \ldots \ldots \ldots \ldots \ldots \ldots$ B-8

B.2.5 Groundwater System $\ldots \ldots \ldots \ldots \ldots \ldots \ldots \ldots \ldots$ B-8

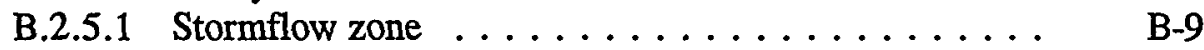

B.2.5.2 Vadose zone ................ B-10

B.2.5.3 Groundwater zone ................ B-11

B.2.5.4 Aquiclude ......................... B-13

B.2.5.5 Aquifer characteristics ............. B . B

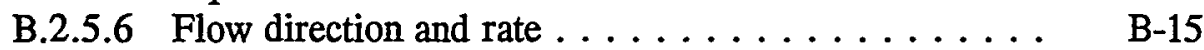

B.2.5.7 Recharge/discharge relationship ......... B-17

B.2.5.8 Groundwater quality ............... B-17

B.2.5.9 Groundwater use ................ B . . . . . . . .

B.3 POST-CLOSURE WASTE MANAGEMENT AREA ... . . . . . . B-19

B.4 CONTAMINANT PLUME DESCRIPTION . . . . . . . . . . . . . B-19

B.5 GROUNDWATER PROTECTION $\ldots \ldots \ldots \ldots \ldots \ldots \ldots$ B-21

B.5.1 Monitoring Parameters . . . . . . . . . . . . B

B.5.2 Groundwater Monitoring System . . . . . . . . . . . B-24 


\section{CONTENTS (continued)}

Page

B.5.3 Sampling and Analysis Plan .................. . . . . . . . . . . . .

B.5.3.1 Sample collection . . . . . . . . . . . B

B.5.3.2 Sample containers, preservation, and transportation . . B-25

B.5.3.3 Laboratory analytical procedures . . . . . . . . B

B.5.3.4 Chain-of-custody control . . . . . . . . . B B-26

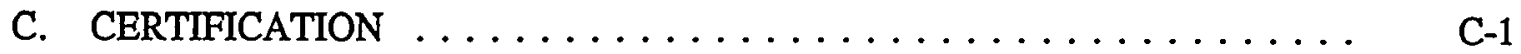

D. REFERENCES ........................ D 


\section{TABLES}

A-1 Typical wastes treated in Kerr Hollow Quarry

A-2 Kerr Hollow Quarry sediment samples taken from 1990 to 1992 (Constituents with total concentration less than detection limit)

A-3 Kerr Hollow Quarry sediment samples taken from 1990 to 1992 (Summary of chemical concentrations with at least one result greater than the detection limit)

A-4 Kerr Hollow Quarry sediment samples taken from 1990 to 1992 (Summary of radionuclide concentrations)

A-5 Kerr Hollow Quarry sediment samples taken from 1990 to 1992 (Analytes with TCLP results less than detection limit)

A-6 Kerr Hollow Quarry sediment samples taken from 1990 to 1992 (Analytes with at least one TCLP result greater than the detection limit)

B-1 Construction data for groundwater monitoring wells located at Kerr Hollow Quarry

B-2 Kerr Hollow Quarry drinking water parameter results for CY 1986

B-3 Kerr Hollow Quarry interim status detection monitoring parameters

B-4 Summary of statistically significant results at the Kerr Hollow Quarry for 1987 through 1994

B-5 Kerr Hollow Quarry 1988 Appendix IX constituent detections

B-6 Summary of field and laboratory residuum permeability test results

B-7 Summary of packer test results for core holes GW-131, GW-135, and CH-157

B-8 Summary of average cation and anion concentrations detected in groundwater samples collected during CY 1994 from Kerr Hollow Quarry monitoring wells

B-9 Public users of groundwater within 10 miles of the Kerr Hollow Quarry

B-10 Validated VOC results from Kerr Hollow Quarry well sampling since first quarter CY 1990 (in $\mu \mathrm{g} / \mathrm{L}$ )

B-11 Monitoring parameters and comparative values

B-12 Construction details of proposed monitoring well network for Kerr Hollow Quarry 


\section{FIGURES}

A-1 Regional Location Map

A-2 Security Map

B-1 Location of groundwater monitoring wells at Kerr Hollow Quarry

B-2 Physiographic Map of Tennessee

B-3 Geology in the Vicinity of Kerr Hollow Quarry

B-4 Generalized geologic map of the Y-12 Plant area

B-5 Geologic cross section through the Chestnut Ridge Hydrogeologic Regime

B-6 Kerr Hollow Quarry Surface Drainage Map

B-7 1993 precipitation data at the NOAA site

B-8 Schematic profile of hydrostratigraphic units in the Chestnut Ridge Hydrogeologic Regime

B-9 Wells used in the packer tests of the Copper Ridge Dolomite

B-10 Water table map for Kerr Hollow Quarry for conditions on August 29, 1994

B-11 Hydrographs for water table wells at Kerr Hollow Quarry, CY 1994

B-12 Hydrographs for Wells GW-147 and GW-231, northwestern well cluster at Kerr Hollow Quarry, CY 1994

B-13 Hydrographs for Wells GW-145 and GW-146, southwestern well cluster at Kerr Hollow Quarry, CY 1994

B-14 Hydrographs for Wells GW-143 and GW-144, southern well cluster at Kerr Hollow Quarry, CY 1994

B-15 Hydrographs for Wells GW-147 and GW-231, northwestern well cluster at Kerr Hollow Quarry, CY 1986 through CY 1993

B-16 Hydrographs for Wells GW-145 and GW-146, southwestern well cluster at Kerr Hollow Quarry, CY 1986 through CY 1993

B-17 Hydrographs for Wells GW-143 and GW-144, southern well cluster at Kerr Hollow Quarry, CY 1986 through CY 1993

B-18 Groundwater geochemistry in the Chestnut Ridge Hydrogeologic Regime

B-19 Location of proposed groundwater monitoring well network at Kerr Hollow Quarry 


\section{APPENDICES}

APPENDIX A RCRA CLOSURE/POST-CLOSURE PLAN FOR THE KERR HOLLOW QUARRY

A.1 REVISED CLOSURE PLAN FOR KERR HOLLOW QUARRY AT THE OAK RIDGE Y-12 PLANT, OAK RIDGE, TENNESSEE

A.2 KERR HOLLOW QUARRY POST-CLOSURE PLAN

A.3 CLOSURE CERTIFICATION LETTER

APPENDIX B WELL CONSTRUCTION DIAGRAMS

APPENDIX C APPLICABLE STANDARD PRACTICE PROCEDURES FOR THE GROUNDWATER PROTECTION PROGRAM

C.1 WELL PLUGGING AND ABANDONMENT

C.2 WELL INSPECTION PROCEDURE

C.3 WELL DEPTH MEASUREMENT PROCEDURE

APPENDIX D SAMPLING AND ENVIRONMENTAL SUPPORT DEPARTMENT OPERATING PROCEDURE - GROUNDWATER SAMPLING

APPENDIX E FALSE-POSITIVE DEMONSTRATIONS

APPENDIX F TRACE METAL EVALUATIONS AND TOLERANCE LIMIT DEMONSTRATIONS FOR GROUNDWATER AT KERR HOLLOW QUARRY 


\section{PLATES}

A-1 Topographic map

A-2 Survey plat map

A-3 Floodplain map 


\section{ACRONYMS}

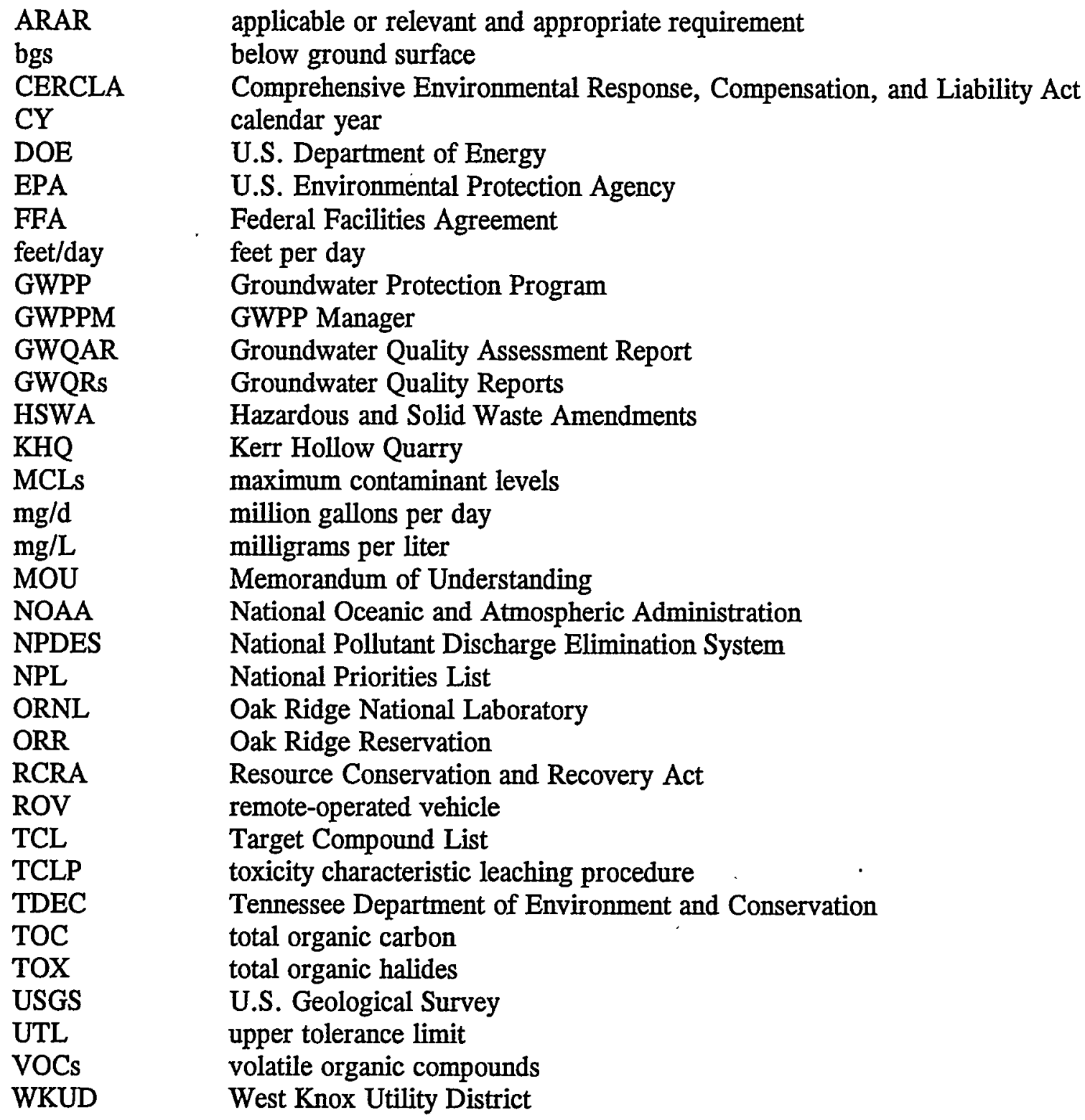


A. GENERAL INFORMATION 


\section{A.2 WASTE CHARACTERISTICS}

Ref: $\quad 1200-1-11-.07(5)(a)$

40 CFR 270.14(b)(2)

The estimated total weight of hazardous and nonhazardous wastes sent to KHQ since 1962 (no records were kept for treatment or disposal in the quarry before 1962) is approximately 50 tons. Types of wastes disposed of in the quarry are identified in Table A-1.

As described in Section A.1, the waste types treated in the KHQ were largely reactive in nature, and consisted of water-reactive materials and potentially explosive chemicals. Treatment in the quarry of these reactive waste types resulted in rendering the wastes nonreactive. As a result of the time period during which treatment (from 1951 to 1988) in the KHQ occurred and the reactive nature of the wastes treated, no analytical data are available for the wastes treated in the KHQ. Furthermore, because of the nature of treatment and closure activities that occurred at the KHQ, no reactive wastes remained after closure. In lieu of analytical data representative of wastes prior to treatment in the KHQ, this section presents the results of sediment sample analysis that provide an indication of the nature of any treatment residues remaining in the KHQ after closure activities were completed.

Between 1990 and 1992, several sampling events specifically targeting sediments in the KHQ were conducted. Tables A-2 through A-6 present a summary of the data collected for the KHQ sediments. Tables A-2 and A-3 present a list of analytes and parameters in total concentrations. Table A-2 specifically illustrates analytes that were sampled for but were not detected above the detection limit. Table A-3 shows those analytes that were detected above the detection limit and the number of detections versus the total number of samples analyzed for the targeted compound and/or parameter. Table A-4 is a summary of the radionuclides found in the KHQ sediment. Tables A-5 and A-6 present a summary of analyses using the toxicity characteristic leaching procedure (TCLP) method for sample preparation. Table A-5 shows those analytes that were analyzed for but not detected above the detection limit and Table A-6 shows those analytes that were detected by the TCLP method. Analytical results indicate that residual contamination is present in low concentrations within the KHQ sediments. In general, hazardous waste constituents were detected at very low levels, if at all. Although several toxicity characteristic metals were detected by total analysis, subsequent analysis using the TCLP method yielded no concentration in excess of regulatory thresholds. 


\section{A.3 FACILITY LOCATION}

Ref: $\quad 1200-1-11-.07(5)(a)$

40 CFR 270.14(b)(11)

The KHQ, occupying about 3 acres, is located along the south side of Chestnut Ridge and in an area located within Y-12 Plant grid coordinates E 63000 to E 64000 and N 24000 to N 25000 (Plates A-1 and A-2). The quarry is about 11/2 miles south of the Y-12 Plant site and $\sim 1,000$ feet north of Bethel Valley Road. Local relief is $\sim 100$ feet with elevations ranging from just under 840 feet above mean sea level at the southwest end of the quarry to over 940 feet on the cliffs immediately northeast of the quarry.

The elevation of the 100-year floodplain for the Clinch River (Melton Hill Lake) is separated in elevation from the KHQ by $\sim 40$ feet. Therefore, the KHQ is not located within a 100 -year floodplain (Plate A-3). The floodplain elevation of 796 feet mean sea level was interpolated from Tennessee Valley Authority floodplain maps for the intermittent stream that enters the river at river mile 41.19. During periods of high precipitation, KHQ overflows and enters the local surface water system. Immediately upon leaving the quarry, the water enters a sediment collection basin that was constructed to mitigate sediment discharge during remedial activities at the quarry. From the basin, the water enters a nearby stream that flows for $\sim 0.9 \mathrm{~km}$ (0.56 mile) then discharges directly into Melton Hill Lake.

\section{A.4 TRAFFIC PATTERNS}

Ref: $\quad 1200-1-11-.07(5)(a)$ 40 CFR 270.14(b)(10)

The Y-12 Plant is served by three highways. The highway system in the area is adequate to handle the average number of daily commuters. The major vehicle access routes to the Y-12 Plant are via Bear Creek Road and Scarboro Road. Bear Creek Road runs east and west along the northern side of the Y-12 Plant. Bear Creek Road is closed for all but official uses, and guard portals are installed along the road. Scarboro Road runs north and south from the Y-12 Plant past the Scarboro facility and intersects with Bethel Valley Road, the main route to Oak Ridge National Laboratory (ORNL) (Plate A-1). Access to KHQ is via an unpaved road that connects to Bethel Valley Road. Scarboro and Bethel Valley Roads are state highways located within the DOE Oak Ridge Reservation (ORR). 
The anticipated volume of traffic associated with $\mathrm{KHQ}$ is minimal and completely dependent upon the required maintenance activities. Light duty service trucks will be primarily used for maintenance purposes.

\section{A.5 SECURITY PROCEDURES AND EQUTPMENT}

Ref: $\quad 1200-1-11-.07(5)(a)$

$$
40 \text { CFR 270.14(b)(4) }
$$

The Oak Ridge Y-12 Plant is a DOE facility that produces and handles nuclear materials. There are security procedures and equipment that will minimize the possibility for unauthorized entry into the facility. The Y-12 Plant has 24-hour surveillance and security, which includes patrol of the area where the KHQ is located. All access roads contain lockable gates to prevent unauthorized entry. The area enclosed by the security fence is shown on Figure A-2. The KHQ is posted with signs warning against unauthorized entry. No languages other than English are necessary for the signs and all signs are legible from a distance of at least 25 feet.

\section{A.6 INSPECTION SCHEDULE}

Ref: $\quad 1200-1-11-.07(5)(a)$

40 CFR 270.14(b)(5)

$1200-1-11-.06(2)(a)$

40 CFR 264.15

Inspections of the KHQ will be conducted to ensure that the integrity of security equipment and groundwater monitoring systems is maintained. The inspections will identify any malfunctions or damage to monitoring wells or equipment, safety equipment, or security devices associated with post-closure care of the property. Throughout the post-closure care period all security and safety components of the KHQ (including well heads) will be inspected quarterly. A comprehensive inspection of monitoring wells will be performed annually.' Notification of damage or need of maintenance of the KHQ monitoring wells will be made to the Y-12 Plant Groundwater Protection Program (GWPP). All inspection records and other documentation for the KHQ will be maintained within the Y-12 Plant. 


\section{A.7 TRAINING}

Ref: $\quad 1200-1-11-.07(5)(a)$

40 CFR 270.14(b)(12)

$1200-1-11-.06(2)(a)$

40 CFR 264.16

Training will be provided to employees conducting inspections to satisfy the requirements of the post-closure permit. The Y-12 Plant GWPP Manager (GWPPM) or authorized designee will train the field personnel who will perform the annual monitoring well inspections to familiarize them with: (1) the purpose and objectives of well inspection and maintenance, (2) well inspection items, (3) well inspection procedure(s), (4) well-depth measurement procedure(s), (5) the proper completion of inspection documentation, and (6) conditions that require notification of the Y-12 Plant GWPPM. Training of field personnel will be conducted prior to program initiation and updated on an annual basis.

On-the-job training will be provided to the site inspectors in the following areas:

- examination of signage;

- examination of monitoring well heads and benchmarks;

- identifying unauthorized deliveries or dumpings; and

- examination of security devices (e.g., fencing, gates, and locks).

A checklist will be used to document inspection findings. Copies of the completed inspection checklist(s) will be maintained at the Y-12 Plant. Examples of the checklists to be used during the inspection(s) are included in the KHQ Post-Closure Plan found in Appendix A.2.

\section{A.8 CLOSURE PLAN AND POST-CLOSURE ACTIVITIES}

Ref: $\quad 1200-1-11-.07(5)(a)$

40 CFR 270.14(b)(13)

The closure plan for the KHQ is located in Section A.1 of Appendix A. The approved closure plan describes performance standards and activities conducted during the closure activities at KHQ. On February 22, 1995, TDEC certified the KHQ closed in accordance with the approved closure plan. A copy of this letter is included in Section A.3 of Appendix A. 
The closure activities conducted pursuant to the approved closure plan included removal of visible containers or structures found within the KHQ. The Closure Certification Report (Dames and Moore 1993) submitted to TDEC detailed the removal activities conducted and included a discussion of post-closure activities. The post-closure activities described in the Closure Certification Report and Post-Closure Plan included care and maintenance of the fencing and posting. Monitoring of the creek that includes the discharge from the KHQ outfall will be conducted as required by the National Pollutant Discharge Elimination System (NPDES) permit TN0002968. The monitoring point is located south of Bethel Valley Road. The purpose of monitoring activities at the $\mathrm{KHQ}$ is to ensure that closure activities were effective and that no impact(s) to groundwater occur in the future. Groundwater monitoring activities will be conducted as described in Section B.5 of this permit application.

\section{A.9 CONTINGENCY PLAN}

Ref: $\quad 1200-1-11-.07(5)(a)$

40 CFR 270.14(b)(7)

A Contingency Plan has been prepared for hazardous waste treatment, storage, and disposal units at the Oak Ridge Y-12 Plant (Skaggs 1993).

\section{A.10 NOTICES REQUIRED FOR DISPOSAL FACILITIES}

$$
\text { Ref: } \quad \cdot 1200-1-11-.07(5)(\mathrm{a})
$$

40 CFR 270.14(b)(14)

$$
1200-1-11-.06(7)(\mathrm{a})
$$

40 CFR 264.119

Within 90 days after completing closure, DOE submitted to the Anderson County, Tennessee Zoning Authority and TDEC a survey plat, prepared and certified by a professional land surveyor, showing the location and dimensions of KHQ with respect to permanently surveyed benchmarks. A copy of the survey plat is included as Plate A-2. A record of the types and approximate quantities of waste placed in the quarry was submitted based on best available knowledge and records.

The DOE ORR property does not have a deed. If, in the future, any Y-12 Plant property on which hazardous waste has been managed is to be released from Federal government 
ownership for private development, information regarding these activities will be placed in a legal document that would be examined during a title search.

\section{A.11 CLOSURE AND POST-CLOSURE COST ESTIMATES}

Ref: $\quad$ 1200-1-11-.07(5)(a)

40 CFR 270.14(b)(15-16)

$1200-1-11-.06(8)(a)$

40 CFR 264.142 to 264.145

As stated in Tennessee Rule 1200-1-11-.06(8)(a) and 40 CFR 264.140(c), Federal facilities are exempt from the requirements for closure and post-closure cost estimates of the abovereferenced regulations.

\section{A.12 TOPOGRAPHIC MAP}

Ref: $\quad$ 1200-1-11-.07(5)(a)

40 CFR 270.14(b)(19)

A topographic map of the KHQ fulfilling all the requirements of the above-referenced regulations is provided on Plate A-1.

\section{A.13 LIABILITY INSURANCE}

Ref: $\quad 1200-1-11-.07(5)(a)$

40 CFR 270.14(b)(17)

As stated in 1200-1-11-.06(8)(a) and 40 CFR 264.140(c), Federal facilities are exempt from the liability insurance requirements of the above-referenced regulations.

\section{A.14 POST-CLOSURE CONTACT}

Ref: $\quad 1200-1-11-.07(5)(a)$

40 CFR 270.14

The address and phone number of the office to contact regarding the KHQ during the postclosure period are listed below:

U.S. Department of Energy, Environmental Restoration Division

P.O. Box 2001

Oak Ridge, Tennessee 37831-8012

(615) 576-0715 
B. GROUNDWATER INFORMATION 


\section{B. GROUNDWATER INFORMATION}

Ref: $\quad 1200-1-11-.07(5)(c)$

40 CFR 270.14(c)

$1200-1-11-.06(6)(a)$

40 CFR 264 Subpart F

\section{B.1 SUMMARY OF INTERIM STATUS DATA}

Ref: $\quad 1200-1-11-.07(5)(c)$

40 CFR 270.14(c)(1)

In 1983, a Memorandum of Understanding (MOU) was signed by DOE, the U.S. Environmental Protection Agency (EPA), and TDEC (HSW, Inc. 1993). The MOU required DOE to initiate a remedial investigation of groundwater contamination at the Y-12 Plant, and characterization monitoring was employed to evaluate the extent of contamination in soils, stream sediments, groundwater, and surface water. The KHQ was granted interim status under RCRA in 1986 (HSW, Inc. 1995). In November 1989, the ORR was placed on the Comprehensive Environmental Response, Compensation, and Liability Act (CERCLA) National Priorities List (NPL) (EPA 1992). On January 1, 1992, DOE, TDEC, and EPA entered into a Federal Facilities Agreement (FFA) for the ORR to provide a framework for conducting assessment and remediation of past and present releases on the ORR.

In February 1986, interim status detection monitoring was initiated at KHQ as required under Part 1200-1-11-.07(5)(c)1 of the Rules Governing Hazardous Waste Management in Tennessee (Jago 1995). Background groundwater quality in each monitoring well at the site was established through quarterly monitoring during 1986 . Semi-annual monitoring for indicator parameters and annual monitoring for water-quality parameters began in 1987. As required under Part 1200-1-11-.05(6)(d)2, analytical results from 1987 and subsequent monitoring years were statistically compared to corresponding background values. Interim status detection monitoring is ongoing at the site.

\section{B.1.1 Monitoring Well Network}

Seven monitoring wells, constructed to RCRA specifications, were installed around the KHQ site in 1985. These wells are designated as GW-142, GW-143, GW-144, GW-145, GW-146, GW-147, and GW-231 (Figure B-1). An eighth boring at the site (CH-143) was drilled as an 
exploratory core hole used for subsurface characterization. This core hole was not completed as a well and was plugged during drilling operations at the adjacent well sites (Jago 1995). A summary of construction data and aquifer zones monitored by the KHQ wells is presented in Table B-1. Monitoring wells GW-142, GW-143, GW-144, GW-145, GW-146, GW-147, and GW-231 comprise the interim status detection monitoring network for KHQ.

Monitoring wells GW-147 and GW-231 (Figure B-1) form a cluster at the northwestern edge of KHQ that extends through unconsolidated material and the top of bedrock. The remaining wells monitor various fractures and fracture zones that were identified in the exploratory core hole. Monitoring wells GW-143 and GW-144 form a cluster south of KHQ to investigate several small fractures zones occurring in two, 50-foot intervals within shallow bedrock. Monitoring wells GW-145 and GW-146 form a cluster to the southwest of the site designed to investigate fracture zones within both shallow and deep bedrock intervals. Monitoring well GW-142 also monitors several fracture zones within shallow bedrock.

\section{B.1.2 Groundwater Sampling and Analysis}

Interim status detection monitoring at KHQ began in 1986 . The first-year data were collected and used to establish a baseline for the monitoring system. This information was reported to TDEC (Energy Systems 1987). The baseline data were used for statistical comparison of data acquired through subsequent sampling efforts. Drinking water parameters compiled from calendar year (CY) 1986 sampling results are presented in Table B-2. During CY 1987, the wells were monitored semi-annually for parameters used as indicators of groundwater contaminations as required by TDEC Rule 1200-1-11-.05(6)(c)(2)iii and annually for parameters establishing groundwater quality as required by TDEC Rule 1200-1-11-.05(の)(c)(2)ii. A summary of parameters analyzed as part of the detection monitoring program is presented in Table B-3. The results of the monitoring effort in CY 1987 were submitted to TDEC (Energy Systems 1988). During CY 1988 and CY 1989, the wells were monitored semi-annually for parameters used as indicators of groundwater contamination. These results were reported to TDEC in required annual reports (Energy Systems 1989, 1990).

During CYs 1990, 1991, 1992, 1993, and 1994, the wells were monitored semi-annually for parameters used as indicators of groundwater contamination. These results were provided

to TDEC in the form of annual and/or semi-annual reports as required under the interim status 
groundwater monitoring requirements (Energy Systems 1991; Shevenell and Switek 1992; McMahon and Mercier 1992; Jago et al. 1993; Jago 1994, 1995).

Additionally, in CY 1990, the wells were incorporated into the Y-12 Plant Comprehensive Groundwater Monitoring Program. In CY 1991, the Groundwater Quality Assessment Report (GWQAR) for the Chestnut Ridge Hydrogeologic Regime (HSW, Inc. 1992) was expanded to include monitoring data throughout the regime to provide a comprehensive picture of groundwater quality throughout the regime. The GWQARs have been provided to TDEC in addition to the annual report that specifically deals with groundwater at the KHQ. As a result of this action, additional locations were sampled and data collected on a quarterly basis, which exceeded RCRA interim status requirements.

In CY 1993, the titles of the GWQARs were changed to Groundwater Quality Reports (GWQRs) to reflect the integration of multiple regulatory, DOE Order, and programmatic requirements into the scope of the Y-12 Plant Comprehensive Groundwater Monitoring Program. The GWQRs continue to be prepared consistent with RCRA interim status assessment reporting requirements. The documents include data necessary to comply with RCRA post-closure permit annual reporting requirements as well as serve as a forum to present data collected under a variety of additional monitoring drivers.

Indicator parameter data from KHQ have been assessed semi-annually using t-test statistical evaluations since the inception of interim status detection monitoring. Formal false-positive demonstrations were prepared in late 1991 (Haase et al. 1991a and 1991b). An initial falsepositive demonstration was submitted that pertained only to $\mathrm{pH}$ and conductivity. A subsequent false-positive demonstration was submitted for total organic carbon (TOC) and total organic halides (TOX). Data pertaining to $\mathrm{pH}, \mathrm{TOC}$, TOX, and specific conductance from the groundwater monitoring wells surrounding the KHQ site were reviewed to determine if the pervasive, statistically significant changes in these parameters obtained using t-test statistics were truly indicative of contaminant releases from KHQ. The false-positive demonstration reports were submitted to TDEC in September 1991 and November 1991, respectively, and were subsequently approved (Lagan 1992). The false-positive demonstrations confirmed that, in CY 1991, statistically significant changes in indicator parameters $\mathrm{pH}, \mathrm{TOC}$, TOX, and specific 
conductance did not represent a release of contaminants to groundwater from the KHQ site. The false-positive demonstrations are included in Appendix E.

Since 1991, several statistically significant changes resulting from the t-test statistical method have been identified. These statistically significant results have also been considered falsepositives based on the premise of the approved false-positive demonstration and historical monitoring data, and do not represent a release of contaminants to the groundwater. The variability of groundwater chemistry over this same time period further emphasizes the conclusions of the false-positive demonstration. A summary of the statistically significant results at the KHQ is presented in Table B-4.

In 1988, analysis for Appendix IX parameters was conducted on groundwater samples taken from monitoring wells GW-142, GW-143, GW-144, GW-145, GW-147, and GW-231 at the KHQ. Analyses included metals, volatile organic compounds (VOCs), semivolatile organics, herbicides/pesticides, and field parameters (e.g., pH, specific conductance, etc.). A summary of the constituents detected is shown in Table B-5. These data are provisional because no validation against laboratory or trip blanks has been conducted.

\section{B.2 HYDROGEOLOGIC FRAMEWORK}

Ref: $\quad 1200-1-11-.07(5)(c)$

40 CFR 270.14(c)(2)

\section{B.2.1 Regional Geology}

The KHQ is located in the western portion of the Valley and Ridge Physiographic Province (Figure B-2). The area is comprised of long, narrow ridges underlain by resistant Paleozoic sandstones, siltstones, and siliceous limestones with intermediate valleys of less resistant shales and soluble carbonates (Rodgers 1953). Structurally, the Valley and Ridge is characterized by a series of imbricate thrust faults. The strike of the rocks is toward the northeast and generally parallels the axes of ridges. The dip of the rocks is to the southeast (Moore 1988; Stockdale 1951). 


\section{B.2.2 Stratigraphic Units}

The KHQ is situated in the Mascot Dolomite of the Knox Group and the lower units of the Chickamauga Group. Five formations of the Knox Group-the Copper Ridge Dolomite, Chepultepec Dolomite, Longview Dolomite, Kingsport Formation, and Mascot Dolomite-comprise the southeast slope of Chestnut Ridge. The Ordovician Chickamauga Group underlies Bethel Valley to the southeast and the Cambrian Maynardville Limestone of the Conasauga Group underlies the lower northwest slope of Chestnut Ridge.

\section{B.2.2.1 Unconsolidated deposits}

Unconsolidated deposits in the Chestnut Ridge Regime consist of reddish-brown to yelloworange residuum overlying the Knox Group. The residuum is characteristically slightly acidic, predominantly composed of clays and iron sesquioxides, and contains semicontinuous, relict beds of fractured chert and other lithologic inhomogeneities (such as silt bodies) that provide a weakly connected network through which saturated flow can occur. However, in the vicinity of KHQ, this residuum is relatively thin or nonexistent (Haase et al. 1987).

\section{B.2.2.2 Bedrock geology}

The KHQ is located at the contact between the uppermost Knox Group and basal portion of the Chickamauga Group (Figure B-3). At the quarry site, the contact is an unconformity and exhibits $\sim 50$ to 100 feet of topographic relief. The uppermost Knox Group at the site consists of gray to blue-gray, medium-bedded to massive dolostone with locally abundant nodular-tobedded chert occurring throughout the section. The basal portion of the Chickamauga Group consists of abundant maroon and gray, thin-to-medium bedded siltstone, thin-bedded limestone, and minor-to-rare nodular or bedded chert. Both the upper Knox Group and the basal Chickamauga Group are pervasively jointed, and dip $\sim 40^{\circ}$ to $50^{\circ}$ to the southeast (Haase et al. 1987).

The KHQ lies at the contact between the Knox Aquifer and the ORR Aquitards, which is coincident with the contact between the Knox and Chickamauga Groups (Solomon et al. 1992). Evaluation of geophysical logs and drill core obtained from boreholes drilled at the quarry site suggests that there are thin ( $<1$ to 3 feet), relatively permeable, fractured zones occurring within bedrock to depths of at least 600 feet (Haase et al. 1987). 
Cavities in carbonate bedrock form by dissolution and abrasion within these fractures (Moore 1988). Enlargement of fractures begins with slow dissolution of rock or cementing materials. Openings enlarged above some critical size permit turbulent groundwater flow. Physical erosion by abrasion then increases the rate of cavity enlargement while turbulent flows remove at least part of the resulting detritus. Remaining detritus accumulates at the bottom of the cavity and partially protects the rock surface from further erosion. Most large cavities develop by abrasion and upward sloping. Solution features encountered in the area during drilling typically were mudfilled or associated with abundant muddy groundwater (Haase et al. 1987). Cavities may also occur in zones of higher water velocity in residuum just above the top of bedrock.

Sinkholes in the KHQ area tend to occur in alignments along certain stratigraphic zones of the Knox Group. One such zone can be found along the crest of Chestnut Ridge. Regional data for the Knox Group in the study area suggest the density of active karst subsidence is on the order of 0.06 to 0.11 per square mile with a frequency of occurrence of 0.01 to 0.02 per square mile per year (Ketelle 1987).

\section{B.2.3 Structural Relationships}

The White Oak Mountain thrust sheet underlies Bethel Valley, Chestnut Ridge, and Bear Creek Valley (Rodgers 1953; Moore 1988). The White Oak Mountain thrust fault is one of a series of imbricate thrust faults in the Valley and Ridge Province that trends northeast and dips southeast (Figures B-4 and B-5).

The most pervasive structural features in the Chestnut Ridge Regime are extensional, hybrid, and shear fractures (Solomon et al. 1992). Three major joint orientations are evident: one that roughly parallels bedding, one steeply dipping set that parallels geologic strike (Dreier et al. 1987), and one steeply dipping set oriented perpendicular to strike. Fracture densities ranging from $\sim 1$ to 60 per foot have been observed in rock outcrops near ORNL (Dreier et al. 1987). Most fractures are short, ranging from tenths of inches to a few feet in length (Solomon et al. 1992).

Bedding and joint orientations were measured by Smith et al. (1983) at the Y-12 Plant. Eighty-three planar orientations were measured, and the results indicate that the bedding orientation is fairly consistent at north $48^{\circ}$ east at $35^{\circ}$ dip to the southeast. Joint orientations 
exhibit a wide scatter of northwest dipping strike set joints and conjugate northeast-dipping and southwest-dipping joints. An additional joint set trends approximately north-south in the vicinity of KHQ (Smith et al. 1983).

Penetrative faults, fractures, and joints in carbonate bedrock provide locations for enhanced groundwater flow and are the features that control subsurface flow in the bedrock. Data also indicate that groundwater movement within the bedrock occurs principally within fractures and fracture systems and not within any single principal stratigraphic unit (Smith et al. 1983).

\section{B.2.4 Surface Water System}

The following sections discuss watershed, surface water use, and precipitation in the vicinity of the KHQ.

\section{B.2.4.1 Southeast Chestnut Ridge Watershed}

The KHQ site is located south of the Chestnut Ridge crest, which forms a surface water divide. During periods of high precipitation, KHQ overflows and enters the local surface water system. Immediately upon leaving the quarry, the water enters a sediment collection basin that was constructed to mitigate sediment discharge during the remedial activities at the quarry. From the basin, the water enters a nearby stream that flows for 0.56 mile and finally discharges directly into Melton Hill Lake (Clinch River). Surface water drainage in the vicinity of KHQ is illustrated in Figure B-6. Stream discharge measurements made in the southeast Chestnut Ridge watershed by the U.S. Geological Survey (USGS) indicate the presence of alternating losing and gaining portions of the area streams (Evaldi 1984).

\section{B.2.4.2 Surface water use}

Surface water use within the immediate vicinity of KHQ is supplied by the Clinch River. Several pumping stations are located within 5 miles of the KHQ site. Two of these stations are owned by DOE and provide water for both domestic and industrial use at the Y-12 Plant (upstream), ORNL (downstream), and the City of Oak Ridge. Two additional stations are operated by the West Knox Utility District (WKUD) with a total usage of 4 to 5.5 million gallons per day (mg/d). The WKUD stations supply 16,000 meters (more than 50,000 customers) with water for domestic use (Heidel 1994). 


\section{B.2.4.3 Precipitation data}

The precipitation data for 1993 presented in Figure B-7 were collected from the National Oceanic and Atmospheric Administration (NOAA) Oak Ridge Station located on South Illinois Avenue, $\sim 2$ miles north of KHQ.

Maximum and minimum annual precipitation measurements made near ORNL from 1954 to 1983 are 74 inches and 35.3 inches, respectively (Moore 1988). The mean annual precipitation for the same time period was 52.2 inches. The least amount of precipitation usually occurs between the months of August and October (2.9 to 3.8 inches measured at the NOAA Oak Ridge Station). The greatest amount of precipitation usually occurs between the months of January and March (5.3 to 6.2 inches). According to NOAA Oak Ridge Station records, the monthly extremes are 13.3 inches for January 1954 and 0.5 inch for August 1983. From 1986 to 1988 , the USGS recorded unusually low annual precipitation readings in the ORNL area (34.5 inches in 1986 and 41.7 inches in 1987).

At the ORR, evapotranspiration consumes on average 30 inches of annual precipitation. Approximately $75 \%$ (22.5 inches) of evapotranspiration occurs between the months of April and September (Moore 1988).

\section{B.2.5 Groundwater System}

Solomon et al. (1992) divide the groundwater system underlying the ORR into two basic hydrogeologic units with fundamentally different hydrologic characteristics: the Knox Aquifer and

the ORR Aquitards. The Knox Aquifer consists of the Knox Group and the underlying Maynardville Limestone formation of the Conasauga Group. The remaining formations of the Conasauga Group, the underlying Rome Formation, and the Chickamauga Group form the ORR Aquitards.

Solomon et al. (1992) divide the Knox Aquifer and the ORR Aquitards into four parts: (1) the stormflow zone; (2) the vadose zone; (3) the groundwater zone which is subdivided into water table, intermediate, and deep intervals; and (4) the aquiclude (Figure B-8). The divisions are based on the amount of water transmitted by each subsystem (i.e., flux), which decreases with depth. The flow system is vertically gradational with no discreet boundaries separating the 
subsystems. However, the bulk permeability of the Knox Aquifer is about ten times greater than that of the ORR Aquitards (Solomon et al. 1992).

The stormflow zone occurs in regolith as does much of the vadose zone, although in some areas the vadose zone includes weathered and fresh bedrock. In the bedrock, orthogonal sets of pervious, planar fractures form groundwater flowpaths. Within a fracture, groundwater may flow either downdip, laterally, or in both directions. Changes in flow direction may occur at fracture splits and intersections, and groundwater flowpaths may locally resemble stairsteps in both plan and sectional views (Moore 1989). Intersections of two or more fracture sets form permeable intervals or zones that transmit the bulk of the groundwater flux (Moore and Toran 1992).

Fracture sets comprising the permeable zone in the water table interval are generally nonstratiform, and groundwater generally flows in the direction of maximum hydraulic gradient along multiple, alternative flowpaths (Moore and Toran 1992). Stratiform fracture sets are believed to form permeable zones at deeper intervals in the bedrock, and groundwater in these zones primarily flows in the direction of geologic strike (which may or may not correlate with the maximum hydraulic gradient) toward cross-cutting tributary streams (Moore and Toran 1992). Additionally, the vertical spacing between permeable zones increases with depth, and these zones are poorly connected in three dimensions (Solomon et al. 1992).

\section{B.2.5.1 Stormflow zone}

Investigations in Bethel Valley and Melton Valley near ORNL show that groundwater occurs intermittently above the water table in the ORR Aquitards in a shallow "stormflow zone" that extends from the ground surface to a depth of $\sim 6$ feet (Moore 1989). Channels for lateral flow in the stormflow zone include macropores and mesopores, which are connected voids created by various processes, including biochanneling, cracking, and soil particle aggregation (Moore 1989). This zone is thicker and more permeable in forested areas than in grassy or brushy areas, and is more permeable near the land surface than at deeper levels (Moore 1989). However, a stormflow zone may not exist in areas that have undergone substantial modification, such as capped portions of waste management areas.

Water is present in the stormflow zone during, and for short periods after, significant rainfall events. Most of the water is lost to evapotranspiration and much of the remaining water 
discharges at nearby seeps, springs, and streams. Analysis of infiltrometer test results from Bethel Valley and Melton Valley suggests that the hydraulic conductivity of the stormflow zone averages $\sim 29$ feet per day (feet/day) near the top and 0.1 foot/day near the base (Moore 1989). Lateral flow in the stormflow zone is intermittent, lasting a few days to a few weeks after the occurrence of precipitation. Flow is fairly rapid when the zone is saturated with water but is relatively slow when nearly drained (Moore 1989).

\section{B.2.5.2 Vadose zone}

The vadose zone occurs between the stormflow zone and the water table. The geometric mean depth to the water table in the Knox Aquifer is $\sim 100$ feet. Water is added to the vadose zone by percolation from the stormflow zone and is removed by transpiration and recharge to the water table. The vadose zone is unsaturated except in the capillary fringe above the water table and within wetting fronts during periods of vertical percolation from the stormflow zone (Moore 1989). Most recharge through the vadose zone is episodic and occurs along discrete permeable fractures that become saturated, even though surrounding micropores remain unsaturated (Solomon et al. 1992).

The hydraulic conductivity of the unsaturated residual soils on Chestnut Ridge has been determined from slug tests in wells located at the United Nuclear Corporation Site (Mishu 1982), and in areas several miles west of the Chestnut Ridge Regime (Woodward-Clyde 1984). Little variation was observed with depth, but conductivities determined by field and laboratory tests varied by approximately two orders-of-magnitude for comparable depth intervals. Mean field conductivities ranged from $5.7 \times 10^{-3}$ to $4.9 \times 10^{-1}$ feet/day and mean laboratory conductivities ranged from $2.8 \times 10^{-5}$ to $9.1 \times 10^{-3}$ feet/day. Results of the slug tests are similar to those obtained from infiltrometer tests. Moore (1988) reported a geometric mean hydraulic conductivity of $\sim 0.006$ foot/day for residuum on Chestnut Ridge based on results of infiltrometer studies near ORNL reported by Watson and Luxmoore (1986) and Wilson and Luxmoore (1988).

The hydraulic conductivity of the residuum overlying the Knox Aquifer varies with the degree of saturation (Luxmoore 1982; Daniels and Broderick 1983). Luxmoore (1982) demonstrated that hydraulic conductivity decreases by approximately one order-of-magnitude with a volumetric water content decrease to $90 \%$ of saturation, and two orders-of-magnitude with a volumetric water content decrease to $75 \%$ of saturation. Daniels and Broderick (1983), as 
summarized in Ketelle and Huff (1984), reported that hydraulic conductivity decreases by roughly one order-of-magnitude relative to maximum when saturation is $90 \%$, and three orders-ofmagnitude relative to maximum when saturation is $75 \%$. Ketelle and Huff (1984) also noted that wide variations in soil permeability occur over short lateral distances. These findings are consistent with observations of permeability variation in residual soils found in other karst areas (Quinlan and Aley 1987).

\section{B.2.5.3 Groundwater zone}

Solomon et al. (1992) divide the saturated zone in the Knox Aquifer and ORR Aquitards into water table, intermediate, and deep intervals based on observed changes in hydrologic characteristics and groundwater geochemistry with depth.

\section{Water Table Interval}

A permeable interval 3 to 15 feet thick usually occurs in the transitional horizon between regolith and unweathered bedrock. The water table often occurs within this permeable interval (Solomon et al. 1992). The saturated thickness of this interval changes with cyclic fluctuations in the water table elevation and the interval may be nearly drained during seasonal water table declines. Moore (1989) estimates an average effective porosity of 0.0042 near the water table.

Results of geochemical dating studies of the ORR Aquitards in Melton Valley generally support the concept of a groundwater zone that is most active near the water table and becomes increasingly sluggish with depth (Solomon et al. 1992). Analyses of ${ }^{3} \mathrm{H}:{ }^{3} \mathrm{He}$ ratios in groundwater samples collected from 1 to 6 feet below the water table showed ages ranging from 0.09 to 7.23 years, and the presence of large amounts of ${ }^{4} \mathrm{He}$ in groundwater samples from greater depths [45 to 165 feet below ground surface (bgs)] suggests much greater residence times (Poreda et al. 1988).

\section{Intermediate Interval}

Groundwater in the intermediate bedrock interval occurs in relatively permeable fractures in a relatively impermeable matrix, but permeable fractures are poorly connected (Solomon et al. 1992). Enlarged fractures and cavities are the primary water-producing features and solute transport pathways, and are supplied by seepage through fractures in the rock matrix, which 
outnumber the enlarged fractures and cavities, are interconnected, and provide the continuity for groundwater flowpaths (Moore 1989).

Estimates of the hydraulic conductivity of the intermediate interval in the Knox Aquifer are provided by results of straddle packer tests performed in three core holes in the Chestnut Ridge Regime: two at the east end of the regime near the Sediment Disposal Basin and one at the west end of the regime near Industrial Landfill IV. The tested intervals were in the Copper Ridge Dolomite of the Knox Group and were generally $<600$ feet bgs. Calculated hydraulic conductivities ranged from 0.0002 to 3.1 feet/day (King and Haase 1988) and averaged $\sim 0.6$ foot/day. The upper population represents more permeable water-producing intervals and the lower population represents less permeable matrix intervals.

The hydraulic conductivity of some water-producing intervals in the Knox Aquifer is much greater than indicated by the straddle packer tests owing to an interconnected network of solution cavities and solutionally enlarged fractures. For instance, groundwater flow rates determined from a tracer test performed by Ketelle and Huff (1984) ranged from 490 to 1,250 feet/day. Preliminary results of a dye-tracer test performed by Geraghty \& Miller, Inc. (1990) at the Security Pits indicated flow rates of $\sim 100$ to 300 feet/day, although findings of the test were not confirmed by a second test performed at the site (SAIC 1993). However, results of the Ketelle and Huff (1984) and the Geraghty \& Miller, Inc. (1990) tracer tests are within the range of conduit-flow rates typical in karst terrains (Quinlan and Ewers 1985).

\section{Deep Interval}

Identification of the deep interval is based on hydraulic and geochemical data for several deep (>500 feet) monitoring wells and core holes. Solomon et al. (1992) report that the boundary between the intermediate interval and deep interval in Bear Creek Valley occurs at depths of $\sim 325$ feet in the ORR Aquitards and 650 feet in the Knox Aquifer. The boundary is based on lower hydraulic conductivity, although use of hydraulic conductivity may not always be a reliable indicator (Solomon et al. 1992).

In some areas of the Bear Creek Regime, the transition from the intermediate interval to the deep interval in the ORR Aquitards is indicated by a change from sodium-bicarbonate groundwater to sodium-chloride groundwater accompanied by a general increase in specific 
conductance and total dissolved solids. This change in groundwater geochemistry probably reflects longer residence times in the deep interval, and usually occurs at depths of $>400$ feet bgs (Solomon et al. 1992). However, no such change in groundwater geochemistry is generally observed in the Knox Aquifer. Within this aquifer, calcium-magnesium-bicarbonate or calciumsulfate groundwater occurs to depths of 1,000 feet bgs (Dreier et al. 1993).

Evaluation of data obtained from straddle packer tests and slug tests, and slow-recovery analyses, indicates that the geometric mean hydraulic conductivity of the deep interval is $\sim 0.001$ foot/day (Solomon et al. 1992). The low conductivities may reflect reduced fracture apertures or increased fracture spacings (Solomon et al. 1992).

\section{B.2.5.4 Aquiclude}

The aquiclude occurs below the deep groundwater interval and is generally marked by the presence of saline water with total dissolved solid concentrations of 40,000 to 300,000 milligrams per liter (mg/L) (Solomon et al. 1992). Information obtained southeast of the Chestnut Ridge Regime in Melton Valley indicates that the saline water typically occurs at depths of $\sim 600$ to 700 feet bgs (Solomon et al. 1992). The water in the aquiclude is a sodium-, calcium-, and chloride-rich brine that is chemically similar to brines associated with major sedimentary basins (Solomon et al. 1992).

Saline groundwater has not been encountered at depth in the Chestnut Ridge Regime. However, several wells completed at depths of $\sim 500$ to 1,000 feet bgs in Bear Creek Valley monitor groundwater with total dissolved solid concentrations of several thousand $\mathrm{mg} / \mathrm{L}$ to several tens of thousand $\mathrm{mg} / \mathrm{L}$, suggesting that the aquiclude may be present at greater depth near the Y-12 Plant than in Melton Valley (Solomon et al. 1992).

\section{B.2.5.5 Aquifer characteristics}

The hydraulic properties of the Knox Group along Chestnut Ridge have been estimated by several investigators (King and Haase 1988; Ketelle and Huff 1984; Woodward-Clyde 1984; and Mishu 1982). The permeability of the unconsolidated residuum controls the vertical rate of water movement from the land to the water table. The permeability of the unweathered bedrock controls the rate of groundwater movement in the water table portion of the aquifer, and is likely to be attributable to secondary openings in the bedrock. 
Both laboratory and in-situ tests have been performed to determine the permeability of the unconsolidated material (Woodward-Clyde 1984; Mishu 1982). Moisture-suction tests were performed on unsaturated laboratory samples using thermocouple psychrometers and tensiometers, and permeability tests were performed on unsaturated samples to determine permeability-suction characteristics of the unconsolidated materials. Permeabilities were determined in the field using falling head permeability tests. Typically, laboratory tests yield lower values than field tests because the macropore system can be tested in the field (Ketelle and Huff 1984). Results of these tests are presented in Table B-6.

Permeability studies conducted in the residuum of the Knox Group at a site several miles southwest of KHQ indicated widely variable permeabilities. At depths of 30 feet or less, permeabilities averaged $\sim 1.41 \times 10^{-9}$ feet/day. At depths $>50$ feet, permeability values decreased to $2.11 \times 10^{-11}$ feet/day on average (Woodward-Clyde 1984). Relatively pervious layers within the residuum with permeability values averaging $1.76 \times 10^{7}$ feet/day were identified and are speculated to be continuous over distances of several thousand feet (WoodwardClyde 1984). Results of another study conducted in Knox Group residuum (Mishu 1982) at a nearby site on Chestnut Ridge indicate permeabilities ranging from $2.89 \times 10^{-10}$ feet/day to $2.54 \times 10^{-7}$ feet/day and averaging $7.05 \times 10^{-8}$ feet/day.

Hydraulic conductivity tests of bedrock along Chestnut Ridge have been conducted by Smith et al. (1983) and King and Haase (1988). A hydraulic conductivity of 0.87 foot/day was calculated by Smith et al. in 1983 using slug test data from monitoring well 1095. King and Haase (1988) conducted straddle packer tests in three core holes completed in the Copper Ridge Dolomite of the Knox Group (Figure B-9). In all, 21 intervals ranging in depth from 120 to 615 feet were individually tested in the Copper Ridge Dolomite. Intervals selected for packer testing were chosen to correspond with highly fractured or solutionally altered sections because these zones were thought to be areas of enhanced groundwater flow. Drill core and geophysical logs of the core holes were examined to identify the zones suitable for packer testing.

Field methods used to conduct the packer tests are described in detail in two reports by Golder \& Associates (1987a and 1987b). Essentially, intervals were tested individually within each core hole and hydraulic heads were measured above the top packer, below the bottom packer, and between the packers using pressure transducers. Where appropriate, shut-in data 
were analyzed according to the Horner semilog method (Erloughler 1977) to determine values of hydraulic conductivity for the tested intervals. The hydraulic conductivity values measured in the 21 intervals ranged from $2.08 \times 10^{-4}$ feet/day to 3.2 feet/day (King and Haase 1988). Hydraulic conductivity values for the tested intervals are presented in Table B-7.

\section{B.2.5.6 Flow direction and rate}

Figure B-10 illustrates a water table map' for KHQ, which was generated from data obtained during the August, CY 1994, comprehensive semi-annual water-level monitoring event at the site. Hydrographs for the water table monitoring wells at KHQ are presented in Figure B-11, and those for the three well clusters are presented in Figures B-12 through B-14. These hydrographs are generated using water level data obtained during quarterly groundwater sampling events.

Throughout most of CY 1994, water levels in all water table wells exhibited similar variations, with water levels rising and falling in response to seasonal changes (Jago 1995). Hydrographs of individual well clusters also show patterns similar to those seen in past years (Figures B-15 through B-17). The hydrographs for the northwestern well cluster (GW-147 and GW-231) indicate hydraulic connection between the unconsolidated and shallow bedrock zones. The hydrographs for the southwestern well cluster (GW-145 and GW-146) indicate a downward vertical hydraulic gradient. However, well GW-146 is not well connected to the shallow groundwater system (emphasized by the lack of recharge to the well between sampling events). In the southern well cluster (GW-143 and GW-144), hydraulic connection between the monitoring intervals is observed based on the similar water level response characteristics of the wells. Reversals in vertical gradient observed in the southern well cluster prior to CY 1993 are not present based on the observed differences in hydraulic head over the course of CY 1993 and CY 1994 (Jago 1995).

The water table elevation map illustrated in Figure B-10 represents conditions that existed on August 29, 1994. Because of the very shallow water table gradient at KHQ, piezometric contours are generalized and subject to some uncertainty. Wells GW-142, GW-145, and GW-231 show negligible difference in piezometric elevation during the third calendar quarter when rainfall is lowest (Figure B-11). Well GW-145 is considered as the formal upgradient well at the site; however, variability in the true upgradient direction has been evident over time with wells GW-231 and GW-142, at times, having the highest water table elevations. 
The water table map suggests that there is groundwater flow in a southerly, and locally southwesterly, direction predominantly toward well GW-144, which consistently has the lowest piezometric head. Water level data from other studies suggest that the groundwater table is closely linked to the water level of the quarry and that the quarry reservoir has local control on hydraulic conditions (Haase et al. 1987; Haase et al. 1988; Early and Switek 1989). Although no water level data for the quarry reservoir are available for CY 1994, the configuration of the water table is similar to that observed in CY 1992 and CY 1993 and is consistent with the interpretations of past studies (Shevenell and Switek 1992; Jago et al. 1993; Jago 1994).

On a regional scale, groundwater flow within the Chestnut Ridge Regime is from higher topographic areas toward the lower areas. Ketelle and Huff (1984) in their study of a West Chestnut Ridge site found that in the Copper Ridge Dolomite outcrop belt, the groundwater divide appears to coincide with the topographic divide. In their conceptual model of groundwater flow, flowpaths in the weathered bedrock zone resemble a rectangular or trellis drainage pattern. Flow follows long runs parallel to strike and is diverted by shorter cross-strike channels to other strike-controlled zones or to discharge in a surface stream. Ketelle and Huff based this model on alignment of sinkholes along strike and on knowledge of fracture patterns.

Two types of groundwater flow are typical of karst systems: diffuse flow and conduit flow. Rates for diffuse flow in the Knox Group were determined using the modified Darcy equation given by $\mathbf{v}=\mathbf{K i} / \mathbf{n}$, where $\mathbf{v}$ is the migration rate, $\mathbf{K}$ is the hydraulic conductivity, $\mathbf{i}$ is the horizontal hydraulic gradient, and $\mathbf{n}$ is the effective porosity. Using the range of hydraulic conductivity test results ( 0.002 to 3.1 feet/day) reported by King and Haase (1988) for wells completed in the Knox Group, an effective porosity of 1\% (Smith et al. 1976), and the range of strike-normal and strike-parallel hydraulic gradients for the Chestnut Ridge Regime (0.06 to 0.09 foot/day and 0.02 to 0.05 foot/day, respectively) reported by HSW, Inc. (1995), the Darcy velocity for diffuse flow ranges from $<1$ foot/year to $\sim 30$ feet/year for flow perpendicular to strike, and from <0.5 foot/year to 16 feet/day for strike-parallel flow (HSW, Inc. 1995).

Although the modified Darcy equation can be used to calculate average flow velocities in fractured rock (assuming it is an equivalent porous media), actual velocities in individual fractures may vary over orders-of-magnitude, depending on fracture aperture and wall roughness (Freeze and Cherry 1979). Additionally, the value for effective porosity is representative for fractured 
limestones, but the actual value may be different by more than an order-of-magnitude, which would result in an inversely proportional variation in the Darcy velocity.

Migration rates for conduit flow were inferred from the results of dye tracer tests performed on Chestnut Ridge. Results of the Geraghty \& Miller, Inc. (1990) and Ketelle and Huff (1984) dye tracer tests provide estimates of conduit flow rates in the Knox Group. Although not confirmed by a subsequent dye test (SAIC 1993), results of the Geraghty \& Miller, Inc. (1990) tracer test indicated flow velocities of 100 to 300 feet/day. Ketelle and Huff (1984) determined flow rates of $\sim 490$ to 1,250 feet/day in their tracer test on Chestnut Ridge west of the Chestnut Ridge Regime.

\section{B.2.5.7 Recharge/discharge relationship}

Groundwater recharge at Chestnut Ridge is derived from precipitation, which averages $\sim 55$ inches per year. A majority of all aquifer recharge occurs during the nongrowing season (i.e., between the months of November and April). Recharge on Chestnut Ridge is via vertical percolation through the regolith and probably includes more direct input via sinkholes. Annual recharge is $\sim 2.6$ inches, and it is possible that a portion of the recharge results from delayed unsaturated flow (Solomon et al. 1992). Groundwater discharge pathways from the ridge have not been completely established, but based on knowledge of flow in similar karst systems and the results of dye tracer tests in the Chestnut Ridge Regime, springs, seeps, and streams located along or across strike are the probable discharge points for groundwater (HSW, Inc. 1995).

\section{B.2.5.8 Groundwater quality}

Groundwater geochemistry in the Chestnut Ridge Regime was evaluated through a review of dissolved concentrations of the principal cations (calcium, magnesium, sodium, and potassium) and total concentrations of the principal anions (bicarbonate, chloride, fluoride, nitrate, and sulfate) reported for CY 1993 groundwater samples collected from monitoring wells at KHQ (HSW, Inc. 1994). Dissolved cation concentrations exclude digested cations that do not contribute charge to the groundwater. A summary of average anion and cation analytical results for the KHQ monitoring wells is presented in Table B-8. The general groundwater geochemistry for the Chestnut Ridge Hydrogeologic Regime is illustrated by major ion concentrations shown in Figure B-18. 
Groundwater geochemistry in the Knox Aquifer is nearly uniform throughout the Chestnut Ridge Regime. The chemical composition is typical of water in contact with dolomite, having the characteristically equivalent molar concentrations of calcium and magnesium with bicarbonate as the predominant anion. However, atypical groundwater geochemistry was observed in samples collected from monitoring wells GW-143 and GW-146 at KHQ (HSW, Inc. 1994). Samples from these wells had higher average sodium, potassium, and sulfate concentrations than the samples from the wells with which they are paired (GW-144 and GW-145, respectively).

The chemistry of the samples from wells GW-143 and GW-146 probably reflects the quality of groundwater in less permeable zones within the Knox Aquifer. Wells GW-143 and GW-146 are completed with deeper monitored intervals than GW-144 and GW-145, and the water level in GW-143 and GW-146 recovers much more slowly after the wells are purged during sampling. The midpoint of the monitored interval for well GW-143 ( $\sim 229$ feet bgs) is $\sim 60$ feet deeper than the midpoint in well GW-144 ( $\sim 171$ feet bgs). Similarly, the midpoint of the monitored interval for well GW-146 ( $\sim 220$ feet bgs) is $\sim 122$ feet deeper than the midpoint in well GW-145 ( 98 feet bgs).

These relationships suggest that the higher average sodium, potassium, and sulfate concentrations in the groundwater monitored by wells GW-143 and GW-146 are a function of longer residence times in deeper aquifer zones with less direct hydraulic communication compared to shallower and more permeable zones in the Knox Aquifer. However, comparison of the analytical results for well GW-142 (midpoint of the monitored interval, $\sim 271$ feet bgs) to those for GW-144 suggests that groundwater in fractures monitored by well GW-142 may have a shorter residence time compared to wells GW-143 and GW-146.

\section{B.2.5.9 Groundwater use}

The City of Oak Ridge obtains its industrial and drinking water supplies from surface water. Within an $~ 10$-mile radius of the KHQ site there are eight users of groundwater (Table B-9). More than 100 wells and springs service residential use within a 20 -mile radius of the KHQ site. 


\section{B.3 POST-CLOSURE WASTE MANAGEMENT AREA}

Ref: $\quad 1200-1-11-.07(5)(c)$

40 CFR 270.14(c)(3)

A topographic map and survey plot fulfilling the requirements of the above-referenced regulation are presented in Plates A-1 and A-2. The DOE ORR property line is illustrated on Plate A-1.

\section{B.4 CONTAMINANT PLUME DESCRIPTION}

Ref: $\quad 1200-1-11-.07(5)(c)$ 40 CFR 270.14(c)(4)

A groundwater contaminant plume has not been identified at the KHQ; therefore, the site has remained in detection monitoring under interim status. Contaminants have been detected periodically in the groundwater at the KHQ; however, the contaminants appear to be attributable to the variability in groundwater chemistry in the area or to closure activities conducted between 1990 and 1993. A discussion of the constituents detected in the groundwater and apparent trends is presented below.

Contamination of groundwater at the KHQ has not been confirmed. Statistically significant variances in RCRA contamination indicator parameters have been observed in facility monitoring wells over time (Table B-4). Indicator parameter results were evaluated and false-positive demonstrations were prepared and submitted to the TDEC staff (Haase et al. 1991a and 1991b) and subsequently approved (Lagan 1992). These demonstrations are provided in Appendix E. The false positive demonstrations confirmed that statistically significant variances in RCRA detection monitoring parameters TOC, TOX, $\mathrm{pH}$, and specific conductance do not represent a release of contaminants to groundwater at the sites (Jago 1995). The data evaluation presented in the false positive demonstration showed that TOC and TOX concentrations did not exhibit an identifiable trend or relationship to concentrations in other wells in the monitoring system and could not be correlated to the summed chlorinated and aromatic VOC values found within each well. The evaluation of $\mathrm{pH}$ data showed that temporal variations apparent in downgradient wells were similar to those exhibited by the upgradient well. The baseline data for $\mathrm{pH}$ in well GW-145 and well GW-142 appear to indicate partial contamination associated with fluids remaining in the formation from well construction. Conductivity data trends were found to be similar in well GW-145 and well GW-142. Further, the conductivity values found in well GW-142 were lower 
than those associated with the upgradient well. As shown in the false positive demonstrations included in Appendix E, these indicator parameters are unreliable "indicators" of groundwater contamination.

An evaluation of all trace metal data collected at the site from the first quarter of CY 1990 through CY 1993 was conducted, and the results were reported in the CY 1993 GQWR for the Chestnut Ridge Hydrogeologic Regime, Part 2 (HSW, Inc. 1994). The evaluation indicated that total strontium and total uranium are consistently elevated ( $>50 \%$ of samples) above Y-12 Plant background screening levels typical for groundwater in uncontaminated areas. Well GW-145 has exhibited both total strontium and total uranium, well GW-142 has exhibited total uranium, and well GW-146 has shown total strontium above screening levels. Given that annual average gross alpha and gross beta results have never exceeded primary drinking water standards, the elevated total uranium and strontium observed in these wells may reflect natural levels of these elements in groundwater at this particular site. Isotopic analyses of groundwater samples were conducted in March 1995 to determine if these two constituents had a radiogenic source. The results indicated no elevated radioisotopes of either parameter in groundwater. Other trace metals have also been found in the groundwater in low concentrations; however, they have not been consistently detected. In addition, trace metal constituents detected in groundwater at the KHQ have not exceeded maximum contaminant levels (MCLs), with the exception of nickel and total uranium, which were detected in the upgradient wells at the site.

Trending of VOC analytical results indicates that very low levels of three VOCs (tetrachloroethene, carbon tetrachloride, and chloroform) have been sporadically observed in two wells (GW-142 and GW-144) over time (Table B-10). Carbon tetrachloride was identified in well GW-144 only. The occurrences of these compounds relate very strongly to the period during which underwater shredding, container breaching, and debris removal actions were being conducted at the quarry (September 1990 through October 1993). Preliminary results of analyses conducted in the first and second quarter of 1995 have shown no VOCs in well GW-142. However, preliminary data for well GW-144 showed trace levels of carbon tetrachloride and chloroform for both quarters and tetrachlorethene in the first quarter only. The chloroform detected in the groundwater may be associated with the carbon tetrachloride since it is a common degradation product of carbon tetrachloride. Because organics have been detected in groundwater 
at $\mathrm{KHQ}$, samples will be analyzed for VOCs as a part of the proposed detection monitoring program discussed in Section B.5.

Additional constituents were identified in unvalidated data from an analysis of Appendix IX constituents conducted in 1988. The constituents found in the analysis include barium, chromium, lead, methylene chloride, acetone, chloroform, carbon tetrachloride, benzene, toluene, fluorotrichloromethane, acetonitrile, di-n-butylphthalate, bis-2-ethylhexylphthalate, and beta-BHC. As illustrated in Table B-5, these contaminants were detected in some cases in both upgradient and downgradient monitoring wells. Most of the constituents found in the Appendix IX analysis have not been detected in subsequent samples collected as a part of the interim status groundwater monitoring program.

\section{B.5 GROUNDWATER PROTECTION}

Ref: $\quad 1200-1-11-.07(5)(c)$

40 CFR 270.14(c)(5)

If KHQ is found to be a source of contamination, the priority of the site will be reevaluated under CERCLA, and the regulatory status of the site will be reprioritized and elevated to CERCLA operable unit status. Any remedial measures required at the site will be addressed as part of the CERCLA process. Remedial actions will be performed in accordance with remedial action work plans developed under CERCLA utilizing RCRA as an applicable or relevant and appropriate requirement (ARAR).

To ensure protection of human health and the environment, monitoring wells GW-142, GW-143, GW-144, GW-145, and GW-231 will comprise the detection monitoring network for KHQ and will be sampled on a semi-annual basis. The groundwater samples collected from the detection monitoring wells will be analyzed for the Target Compound List (TCL) presented in Table B-11 and compared to the corresponding tolerance limits to assess if a release of contaminants has occurred. This TCL is comprised of the alternative indicator parameters and comparative values that will be used in the detection monitoring program. The comparative values are based on calculated 95\% upper tolerance limits (UTLs) (barium, lead, uranium) or MCLs (cadmium, chromium, mercury, nickel, carbon tetrachloride, tetrachloroethene, chloroform, gross alpha, and gross beta). In addition, as indicated on Table B-11 and discussed in Section B.5.1, annual trending analysis will be conducted for boron and total strontium. 
Pursuant to Section IV of the FFA entered into by DOE, EPA, and TDEC, and in light of the requirement in the FFA to achieve a cleanup under CERCLA using a risk-based approach that is protective of human health and the environment, corrective action under this permit shall be determined accordingly. After the work called for in the FFA has been completed, the need for any further corrective action will be addressed under CERCLA and shall be limited to action based on new information or conditions, not available at the time of the remedy selected under the FFA, that render the FFA remedy no longer protective of human health and the environment.

\section{B.5.1 Monitoring Parameters}

Alternative indicator parameters will be monitored at the site (Table B-11). Monitoring and reporting frequencies will be semi-annual. Evaluation of groundwater analytical data will be performed by comparing analytical results to the values developed according to the methodology outlined in Appendix F. The results of this comparison will be submitted by the first day of September and March of each year. This evaluation will be conducted by comparing the data against calculated UTLs and MCLs identified in Table B-11 and, for boron and total strontium, annual trending analysis will be performed. The groundwater flow rate and direction in the uppermost aquifer will be determined annually.

Indicator parameters for the proposed detection monitoring program at the KHQ were chosen based upon the wastes treated at the unit and an evaluation of historical groundwater data. The historical data reviewed included data from the Y-12 Plant Groundwater Monitoring Program and the interim status groundwater monitoring efforts at the KHQ. Data from all seven groundwater monitoring wells in the area of KHQ were evaluated. The analytes chosen for the proposed detection monitoring system at the KHQ include: barium, boron, cadmium, chromium, lead, mercury, nickel, strontium (total), uranium (total), gross alpha, gross beta, and selected VOCs of potential concern.

As discussed in Appendix F, UTLs could not be calculated for all parameters of concern. The UTLs were calculated at a $95 \%$ confidence level for barium, lead, and uranium. The UTLs could not be calculated for cadmium, chromium, mercury, or nickel because these contaminants were detected in $<10 \%$ of the samples for which they were analyzed. Because UTLs could not be calculated, the MCLs for these parameters are proposed as the concentrations to be used for comparative purposes. Historical data have shown that MCLs have not been exceeded for these 
proposed trace metal parameters, with the exception of nickel and total uranium, which were detected in the upgradient wells at the site.

In analyzing the historical data, it was noted that boron and strontium were significantly different between individual wells and well groupings (both upgradient and downgradient groups). In addition, strontium is not a hazardous constituent identified in Appendix VIII of 40 CFR Part 261 , nor does it have an MCL. Therefore, it is proposed that no comparative concentration (e.g., 95\% UTL) be set for total strontium. Instead, analysis of groundwater for strontium will be continued as a part of the detection monitoring program and the annual trend of these results will be evaluated to determine whether a significant change in concentrations is seen over time.

Boron concentrations in groundwater at the site were also significantly different between wells in both the upgradient and downgradient well populations, as well as between the two well groupings. Boron concentrations were found to be higher overall in downgradient wells. Because of the variations in concentrations found within the wells, a 95\% UTL was not calculated for this analyte. Although the boron may be attributable to activities at the KHQ, the data do not correspond to a release of any constituent listed in Appendix VIII of 40 CFR Part 261 as incorporated by reference in TN Rule 1200-1-11-.02(5)(a)(1). Because boron is not an Appendix VIII constituent, does not have an associated MCL, and cannot be correlated to a release of any hazardous constituent from the $\mathrm{KHQ}$, it is proposed that no comparative concentration (e.g., 95\% UTL) be established for this constituent. Boron, however, will be used as an indicator parameter and groundwater analytical results will be evaluated by annual trending analysis.

In developing the proposed list of parameters for the KHQ detection monitoring program, it was noted that additional parameters have been sporadically detected over time. Beryllium and silver have been detected in groundwater, but were not included in the proposed parameter list because they have not been detected on a consistent basis and, when detected, have never exceeded associated MCLs.

In addition to the constituents discussed above, historical data have shown that certain VOCs have been detected in the groundwater at low concentrations. The VOCs detected in the groundwater included tetrachloroethene, carbon tetrachloride, and chloroform. These VOCs are believed to be attributable to closure activities. The MCLs have not been exceeded for detected 
VOCs. Because of the detection of these VOCs, it is proposed that groundwater samples at the KHQ be analyzed for these VOCs. Any VOC detected will be compared to the associated MCL as a part of the detection monitoring program, and any observed trends in VOC concentrations will be evaluated.

It is also proposed that gross alpha and gross beta be included in the detection monitoring program at KHQ. These parameters will be compared to MCLs and, if found to exceed the annual average, will be followed by isotopic analysis. This activity will determine whether radionuclides associated with the operation of KHQ are present within the groundwater.

\section{B.5.2 Groundwater Monitoring System}

The proposed groundwater detection monitoring well system includes wells GW-142, GW-143, GW-144, GW-145, and GW-231. Wells GW-142 and GW-231 shall be considered upgradient wells. Wells GW-143, GW-144, and GW-145 are downgradient wells for the site. Aquifer zones monitored by these wells are presented in Table B-12. The locations of wells for the proposed groundwater detection monitoring network are shown on Figure B-19. All of these wells were constructed in accordance with the standards outlined in TN Rule 1200-1-11-.06(6)(a). Well construction diagrams are provided in Appendix B. In addition, construction details of the wells included in the proposed detection monitoring network are also included in Table B-12.

Additional wells may be installed in the area as part of future groundwater investigations under CERCLA or other non-RCRA activities. Construction in the vicinity of KHQ may require that some wells be abandoned and plugged at some point in the future. Prior approval for plugging and abandoning any detection monitoring well will be obtained from TDEC. The most current approved version of the procedure for well plugging and abandonment (Appendix C.1) will be used to decommission any well.

\section{B.5.3 Sampling and Analysis Plan}

The procedures for sampling and analysis for the groundwater monitoring program at KHQ are designed to provide representative results of the groundwater quality. The groundwater elevations will be measured in each well before the samples are collected as described in the most current approved version of the standard operating procedure for groundwater monitoring \{Appendix D [1200-1-11-.06(a)]\}. Wells will be inspected annually according to the most current 
approved version of the procedure provided in Appendix C.2. In addition, well depths will be verified annually in accordance with the most current approved version of the Y-12 Plant GWPP well depth measurement procedure (Appendix C.3).

\section{B.5.3.1 Sample collection}

Groundwater samples from monitoring wells at KHQ will be collected in accordance with the most current approved version of the procedure presented in Appendix D to ensure that the integrity of the samples is not compromised. Samples will be collected using one of three types of sampling apparatus or an equivalent: dedicated bladder pumps, portable gas piston pumps, or bailers.

A discrete sample will be obtained daily from each well for 4 consecutive days. Each well will be purged prior to collection of the four discrete samples, and field parameters will be monitored in accordance with the most current approved version of procedures outlined in Appendix D. The bladder pumps and bailers can be used for both well purging and sample collection. Likewise, the gas piston pumps can also be used for well purging and sample collection; however, these units are not suitable for collecting samples to be analyzed for VOCs. For this reason, all samples to be analyzed for VOCs will be obtained with either a bladder pump or a bailer. The pumps and bailers will be decontaminated after sampling each well, if not dedicated, in accordance with the most current approved version of procedures outlined in Appendix D.

\section{B.5.3.2 Sample containers, preservation, and transportation}

The types of sample containers and preservation methods are contained in the most current approved version of the groundwater sampling and analysis plan (Burnett and Dill 1990). The most current approved version of methods for preservation and transportation of groundwater samples are also presented in Burnett and Dill (1990).

\section{B.5.3.3 Laboratory analytical procedures}

Laboratory methods to be used to analyze the groundwater samples are provided in the most current approved version of the groundwater sampling and analysis plan (Burnett and Dill 1990) or the equivalent of those methods. 


\section{B.5.3.4 Chain-of-custody control}

The current approved protocol for sample chain-of-custody control is presented in Burnett and Dill (1990). 


\section{CERTIFICATION}




\section{CERTIFICATION}

Ref: $\quad 1200-1-11-.07(2)(a) 10$

I certify under penalty of law that this document and all attachments were prepared under my direction and supervision in accordance with a system designed to ensure that qualified personnel properly gather and evaluate the information submitted. Based on my inquiry of the person or persons who manage the system, and those persons directly responsible for gathering the information, the information submitted is, to the best of my knowledge and belief, true, accurate, and complete. I am aware that there are significant penalties for submitting false information, including the possibility of fine and imprisonment for knowing violations.

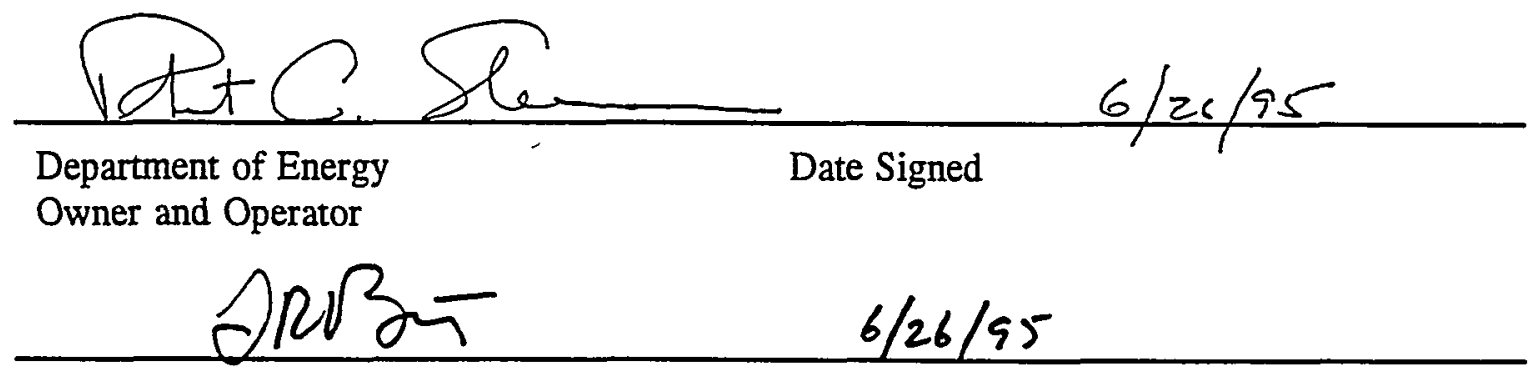

Lockheed Martin Energy Systems, Inc. Co-Operator

Date Signed

The Department of Energy and its operating contractor, Lockheed Martin Energy Systems, Inc., have jointly signed this application as the operator of the permitted facility. The Department has determined that dual signatures best reflect the actual apportionment of responsibility under which the Department's RCRA responsibilities are for policy, programmatic, funding and scheduling decisions, as well as general oversight, and the contractor's RCRA responsibilities are for day-to-day operations, including but not limited to the following responsibilities: waste analysis and handling, monitoring, recordkeeping, reporting, and contingency planning. For purposes of the certification required by Tennessee Rule 1200-1-11-.07(2)(a)10, the Department's and Lockheed Martin Energy Systems, Inc.'s representatives certify, to the best of their knowledge and belief, the truth, accuracy, and completeness of the application for their respective areas of responsibility.

This statement is attached hereto for the purpose of clarifying the roles and responsibilities of DOE and Lockheed Martin Energy Systems, Inc. with respect to the permitted facility and it shall not be construed as altering or limiting the certification. 


\section{REFERENCES}




\section{REFERENCES}

Burnett, L.P. and M.S. Dill 1990. Groundwater Sampling and Analysis Plan. Martin Marietta Energy Systems, Inc. (K/QT-167).

Dames and Moore 1993. Closure Certification Report for Kerr Hollow Quarry at the Oak Ridge Y-12 Plant, Oak Ridge, Tennessee, November 30, Job No. 10805-814-154.

Daniels, D.E. and G. Broderick 1983. Results of Moisture-Suction and Permeability Tests on Unsaturated Samples, ORNL/Sub/83-6476/1, Oak Ridge National Laboratory.

DOE (U.S. Department of Energy) 1993. Revised Closure Plan for Kerr Hollow Quarry at the Oak Ridge Y-12 Plant, Oak Ridge, Tennessee, DOE/OR/01-1103\&D3.

Dreier, R.B., D.K. Solomon, and C.M. Beaudoin 1987. "Fracture Characterization in the Unsaturated Zone of a Shallow Land Burial Facility in: Flow and Transport through Fractured Rock," American Geophysical Union Monograph, p. 42.

Dreier, R.B., T.O. Early, and H.L. King 1993. Results and Interpretations of Groundwater Data Obtained from Multi-port Instrumented Core Holes (GW-131 through GW-135) Fiscal Years 1990 and 1991, Y/TS-803, Martin Marietta Energy Systems, Inc.

Early, T.O. and J. Switek 1989. Annual Report of 1988 Groundwater Monitoring Data for Treatment, Storage, and Disposal Facilities at the Y-12 Plant: Groundwater Surface Elevations, Y/TS-511/P1, Martin Marietta Energy Systems, Inc.

Energy Systems (Martin Marietta Energy Systems, Inc.) 1987. Annual Groundwater Monitoring Datafor Treatment, Storage, and Disposal Facilities at the Y-12 Plant: Statistical Evaluation of First Year Data, Y/TS-242/P2, Martin Marietta Energy Systems, Inc., Oak Ridge Y-12 Plant.

Energy Systems 1988. Annual Groundwater Monitoring Data for Treatment, Storage, and Disposal Facilities at the Y-12 Plant: Reporting and Statistical Evaluation of Subsequent (Second) Year Data, Y/TS-388/P2, Martin Marietta Energy Systems, Inc., Oak Ridge Y-12 Plant.

Energy Systems 1989. Annual Groundwater Monitoring Data for Treatment, Storage, and Disposal Facilities at the Y-12 Plant: Reporting and Statistical Evaluation of the Third Year Data, Y/TS-511/P2, Martin Marietta Energy Systems, Inc., Oak Ridge Y-12 Plant.

Energy Systems 1990. Annual Groundwater Monitoring Data for Treatment, Storage, and Disposal Facilities at the Y-12 Plant: Reporting and Statistical Evaluation of Fourth Year Data, Y/TS-625/P2, Martin Marietta Energy Systems, Inc., Oak Ridge Y-12 Plant.

Energy Systems 1991. Annual Groundwater Monitoring Data for Treatment, Storage, and Disposal Facilities at the Y-12 Plant: Reporting and Statistical Evaluation of the Fifth Year Data, Y/TS-724/P2, Martin Marietta Energy Systems, Inc., Oak Ridge Y-12 Plant. 
EPA (U.S. Environmental Protection Agency) 1992. Federal Facility Agreement for the Oak Ridge Reservation (between the U.S. Environmental Protection Agency - Region IV, the U.S. Department of Energy, and Tennessee Department of Environment and Conservation), DOE/OR-1014.

Erloughler, R.C. 1977. "Advances in Well Test Analysis," American Institute of Mining, Metallurgical and Petroleum Engineers, Monograph Vol. 5 of the Henry L. Doherty Series.

Evaldi, R.D. 1984. Stream flow and Specific-Conductance Data for Selected Sites, February 15 through April 9, 1984, near the Y-12 Plant, the Oak Ridge Reservation, Tennessee, United States Geological Survey, Open-file Report 84-625.

Fraser, A.S., J.H. Vanderlan, and K.L. Walker 1993. Closure Certification Report for the Kerr Hollow Quarry Site at the Oak Ridge Y-12 Plant, Oak Ridge, Tennessee, Y/ER-153, Martin Marietta Energy Systems, Inc.

Freeze, R.A. and J.A. Cherry 1979. Groundwater, Prentice-Hall, Inc., Englewood Cliffs, New Jersey, pp. 408-409.

Geraghty \& Miller, Inc., 1990. A Study of Ground-Water Flow from Chestnut Ridge Security Pits using a Fluorescent Dye Tracer, Y/SUB/90-00206C/6, Prepared for Martin Marietta Energy Systems, Inc.

Golder \& Associates 1987a. Geohydrologic Packer Testing, GW-132, GW-133, GW-134, and GW-135, ORNL Y-12 Plant Area, Consultant's Report, Prepared for the Remedial Action Program at the Oak Ridge Y-12 Plant, October.

Golder \& Associates 1987b. Geohydrologic Packer Testing, GW-131, CH-157, ORNL Y-12 Plant Area, Consultant's Report, Prepared for the Remedial Action Program at the Oak Ridge Y12 Plant, November.

Haase, C.S., H.L. King, G.A. Gillis, C.W. Kimbrough, and T.M. Mercier 1987. Preliminary Assessment of Groundwater Data for the Kerr Hollow Quarry Site at the Y-12 Plant, Oak Ridge, Tennessee, Y/TS-267, Martin Marietta Energy Systems, Inc.

Haase, C.S., G.A. Gillis, and H.L. King 1988. Annual Report of 1987 Groundwater Monitoring Data for Treatment, Storage, and Disposal Facilities at the Y-12 Plant: Groundwater Surface Elevations, Y/TS-388/P1, Martin Marietta Energy Systems, Inc.

Haase, C.S., H.L. King, and T.M. Mercier 1991a. False-Positive Demonstration for Chestnut Ridge Sediment Disposal Basin and Kerr Hollow Quarry, Internal Copy, Martin Marietta Energy Systems, Inc., Oak Ridge Y-12 Plant, September 10.

Haase, C.S., H.L. King, and T.M. Mercier 1991b. Evaluation of Total Organic Carbon and Total Organic Halide Measurements for Groundwater from the Chestnut Ridge Sediment Disposal Basin and Kerr Hollow Quarry, Internal Copy, Martin Marietta Energy Systems, Inc., Oak Ridge Y-12 Plant, November 25. 
Heidel, D. 1994. West Knox Utilities Division. Personal Communication to J. Brown, Geraghty \& Miller, Inc., August 1.

HSW, Inc. (HSW Environmental Consultants, Inc.) 1992. Calendar Year 1991 Groundwater Quality Assessment Report for the Chestnut Ridge Hydrogeologic Regime, Y-12 Plant, Oak Ridge, Tennessee, Y/SUB/92-YP507C/3, Prepared for Martin Marietta Energy Systems, Inc., Oak Ridge Y-12 Plant.

HSW, Inc. 1993. Oak Ridge Y-12 Plant Groundwater Protection Program Management Plan, Y/SUB/93-YP507C/4, Prepared for Martin Marietta Energy Systems, Inc., Oak Ridge Y-12 Plant.

HSW, Inc. 1994. Calendar Year 1993 Groundwater Quality Report for the Chestnut Ridge Hydrogeologic Regime, Y-12 Plant, Oak Ridge, Tennessee, Y/SUB/94-EAQ10C/3/P1\&P2, Prepared for Martin Marietta Energy Systems, Inc., Oak Ridge Y-12 Plant.

HSW, Inc. 1995. Calendar Year 1994 Groundwater Quality Report for the Chestnut Ridge Hydrogeologic Regime, Y-12 Plant, Oak Ridge, Tennessee, Prepared for Martin Marietta Energy Systems, Inc., Oak Ridge Y-12 Plant.

Jago, W.K., T.M. Mercier, and L.A. Shevenell 1993. Annual Report of 1992 Groundwater Monitoring Data for the Kerr Hollow Quarry and Chestnut Ridge Sediment Disposal Basin, Y-12 Plant, Oak Ridge, Tennessee, Y/TS-958, Martin Marietta Energy Systems, Inc., Oak Ridge Y-12 Plant.

Jago, W.K. 1994. Annual Report of 1993 Groundwater Monitoring Data for the Kerr Hollow Quarry and Chestnut Ridge Sediment Disposal Basin, Y-12 Plant, Oak Ridge, Tennessee, Y/TS-1172, Martin Marietta Energy Systems, Inc., Oak Ridge Y-12 Plant.

Jago, W.K. 1995. Annual Report of 1994 Groundwater Monitoring Data for the Kerr Hollow Quarry and Chestnut Ridge Sediment Disposal Basin, Y-12 Plant, Oak Ridge, Tennessee, Y/TS-1287, Martin Marietta Energy Systems, Inc., Oak Ridge Y-12 Plant.

Jones, S.B., S.M. Field, and B.K. Thompson 1994. Updated Subsurface Data Base for Bear Creek Valley, Chestnut Ridge, and Part of Bethel Valley on the U.S. Department of Energy Oak Ridge Reservation, Y/TS-881/R2, June.

Ketelle, R.H. 1987. Internal Correspondence to L.L. McCauley on the subject of Karst Hazards Related to Waste Management, September 29.

Ketelle, R.H. and D.D. Huff 1984. Site Characterization of the West Chestnut Ridge Site, ORNL/TM-9229, Oak Ridge National Laboratory.

King, H.L. and C.S. Haase 1987. Subsurface-Controlled Geological Maps for the Y-12 Plant and Adjacent Areas of Bear Creek Valley, ORNL/TM-10012, Oak Ridge National Laboratory. 
King, H.L. and C.S. Haase 1988. Summary of Results and Preliminary Interpretation of Hydrogeologic Packer Testing in Core Holes GW-131 Through GW-135 and CH-157, Oak Ridge Y-12 Plant, Y/TS-495, Prepared for Martin Marietta Energy Systems, Inc. by E.C. Jordan Company.

King, H.L. 1994. Martin Marietta Energy Systems, Inc. Personal Communication from D. Rosowitz, Martin Marietta Energy Systems, Inc. July.

Lagan, C. 1992. Tennessee Department of Environmental and Conservation, letter to D.J. Bostock, Martin Marietta Energy Systems, Inc., "Approval for False Positive Demonstration for Chestnut Ridge Sediment Disposal Basin and Kerr Hollow Quarry," January 9.

Luxmoore, R.J. 1982. Physical Characteristics of Soils of the Southern Region Fullerton and Sequoia Series, ORNL-5868, Oak Ridge National Laboratory.

McMahon, L.W. and T.M. Mercier 1992. Annual Report of 1991 Groundwater Monitoring Data for the Kerr Hollow Quarry and Chestnut Ridge Sediment Disposal Basin at the Y-12 Plant: Reporting and Statistical Evaluation of the Subsequent Year (Sixth) Data, Y/TS-833/P2, Martin Marietta Energy Systems, Inc., Oak Ridge Y-12 Plant.

Mishu, L. 1982. Subsurface Analysis of Waste Disposal Facilities at the Y-12 Plant, Y/SUB/8224700/2, Prepared for Martin Marietta Energy Systems, Inc. by Geotek Engineering Company.

Moore, G.K. 1988. Concepts of Groundwater Flow and Occurrence Near Oak Ridge National Laboratory, ORNL/TM-10969, Oak Ridge National Laboratory.

Moore, G.K. 1989. Groundwater Parameters and Flow Systems Near Oak Ridge National Laboratory, ORNL/TM-11368, Oak Ridge National Laboratory.

Moore, G.K. and L.E. Toran 1992. Supplement to a Hydrogeologic Framework for the Oak Ridge Reservation, Oak Ridge, Tennessee, ORNL/TM-12191, Oak Ridge National Laboratory.

Poreda, L.N., T.E. Cerling, and D.K. Solomon 1988. "Tritium and Helium Isotopes as Hydrologic Tracers in a Shallow Unconfined Aquifer," Journal of Hydrology 103:1-9.

Quinlan, J.F. and R.O. Ewers 1985. Groundwater Flow in Limestone Terrains: Strategy, Rationale, and Procedures for Reliable, Efficient Monitoring of Groundwater Quality in Karst Areas, National Symposium and Exposition on Aquifer Restoration and Groundwater Monitoring Proceedings, National Water Well Association, Worthington, Ohio, pp. 197-234.

Quinlan, J.F. and T. Aley 1987. "Discussion of a New Approach to the Disposal of Hazardous Waste on Land," Groundwater 25:615-616.

Rodgers, J. 1953. "Geologic Map of East Tennessee with Explanatory Text," Tennessee Division of Geology, Bulletin 58, Part II. 
King, H.L. and C.S. Haase 1988. Summary of Results and Preliminary Interpretation of Hydrogeologic Packer Testing in Core Holes GW-131 Through GW-135 and CH-157, Oak Ridge Y-12 Plant, Y/TS-495, Prepared for Martin Marietta Energy Systems, Inc. by E.C. Jordan Company.

King, H.L. 1994. Martin Marietta Energy Systems, Inc. Personal Communication from D. Rosowitz, Martin Marietta Energy Systems, Inc. July.

Lagan, C. 1992. Tennessee Department of Environmental and Conservation, letter to D.J. Bostock, Martin Marietta Energy Systems, Inc., "Approval for False Positive Demonstration for Chestnut Ridge Sediment Disposal Basin and Kerr Hollow Quarry," January 9.

Luxmoore, R.J. 1982. Physical Characteristics of Soils of the Southern Region Fullerton and Sequoia Series, ORNL-5868, Oak Ridge National Laboratory.

McMahon, L.W. and T.M. Mercier 1992. Annual Report of 1991 Groundwater Monitoring Data for the Kerr Hollow Quarry and Chestnut Ridge Sediment Disposal Basin at the Y-12 Plant: Reporting and Statistical Evaluation of the Subsequent Year (Sixth) Data, Y/TS-833/P2, Martin Marietta Energy Systems, Inc., Oak Ridge Y-12 Plant.

Mishu, L. 1982. Subsurface Analysis of Waste Disposal Facilities at the Y-12 Plant, Y/SUB/8224700/2, Prepared for Martin Marietta Energy Systems, Inc. by Geotek Engineering Company.

Moore, G.K. 1988. Concepts of Groundwater Flow and Occurrence Near Oak Ridge National Laboratory, ORNL/TM-10969, Oak Ridge National Laboratory.

Moore, G.K. 1989. Groundwater Parameters and Flow Systems Near Oak Ridge National Laboratory, ORNL/TM-11368, Oak Ridge National Laboratory.

Moore, G.K. and L.E. Toran 1992. Supplement to a Hydrogeologic Framework for the Oak Ridge Reservation, Oak Ridge, Tennessee, ORNL/TM-12191, Oak Ridge National Laboratory.

Poreda, L.N., T.E. Cerling, and D.K. Solomon 1988. "Tritium and Helium Isotopes as Hydrologic Tracers in a Shallow Unconfined Aquifer," Journal of Hydrology 103:1-9.

Quinlan, J.F. and R.O. Ewers 1985. Groundwater Flow in Limestone Terrains: Strategy, Rationale, and Procedures for Reliable, Efficient Monitoring of Groundwater Quality in Karst Areas, National Symposium and Exposition on Aquifer Restoration and Groundwater Monitoring Proceedings, National Water Well Association, Worthington, Ohio, pp. 197-234.

Quinlan, J.F. and T. Aley 1987. "Discussion of a New Approach to the Disposal of Hazardous Waste on Land," Groundwater 25:615-616.

Rodgers, J. 1953. "Geologic Map of East Tennessee with Explanatory Text," Tennessee Division of Geology, Bulletin 58, Part II. 
TABLES 
Table A-1. Typical wastes treated in Kerr Hollow Quarry

\begin{tabular}{|c|c|}
\hline Waste Type & Examples \\
\hline \multirow{9}{*}{$\begin{array}{l}\text { Alkali metals and metal hydrides } \\
\text { (water reactive) }\end{array}$} & Lithium and lithium hydride \\
\hline & Sodium and sodium hydride \\
\hline & Potassium and potassium hydride \\
\hline & Calcium and calcium hydride \\
\hline & Zirconium hydride \\
\hline & Titanium hydride \\
\hline & Lithium hydride \\
\hline & Lithium aluminum hydride \\
\hline & Sodium-potassium (NaK) metal \\
\hline \multirow[t]{4}{*}{ Unstable organics (explosive) } & Picric acid and related compounds \\
\hline & Ethers \\
\hline & Peroxides \\
\hline & Hydrazine \\
\hline \multirow[t]{2}{*}{ Metals (reactive) } & Phosphorous \\
\hline & Magnesium \\
\hline \multirow[t]{4}{*}{ Mechanical hazards (explosive) } & Stressed steel parts (shrapnel danger) \\
\hline & Vacuum tubes (implosive) \\
\hline & Blasting caps \\
\hline & Gas cylinders (with frozen/broken valves) \\
\hline \multirow[t]{2}{*}{ Miscellaneous hazards } & Ammonia (irritant) \\
\hline & Inorganic acids (corrosive) \\
\hline
\end{tabular}

Source: DOE 1993 
Table A-2. Kerr Hollow Quarry sediment samples taken from 19901992

(Constituents with total concentration less than detection limit)

\begin{tabular}{|c|c|c|c|c|}
\hline Analysis (units) & $\begin{array}{c}\text { Number of } \\
\text { samples }\end{array}$ & $\begin{array}{c}\text { Minimum } \\
\text { detection } \\
\text { limit }\end{array}$ & $\begin{array}{c}\text { Average } \\
\text { detection } \\
\text { limit }\end{array}$ & $\begin{array}{c}\text { Maximum } \\
\text { detection } \\
\text { limit }\end{array}$ \\
\hline \multicolumn{5}{|l|}{ Metals } \\
\hline Arsenic (mg/kg) & 18 & 17 & 26.7 & 35 \\
\hline Gallium (mg/kg) & 18 & 7.4 & 12 & 16 \\
\hline Molybdenum (mg/kg) & 18 & 2.5 & 3.99 & 5.2 \\
\hline Silver (mg/kg) & 18 & 2.5 & 3.99 & 5.2 \\
\hline Thallium (mg/kg) & 2 & 18 & 21 & 24 \\
\hline Thorium (mg/kg) & 18 & 4.1 & 6.66 & 8.7 \\
\hline \multicolumn{5}{|l|}{ Pesticides and PCBs } \\
\hline PCB, Total (mg/kg) & 2 & 1 & 1 & 1 \\
\hline \multicolumn{5}{|l|}{ Semivolatile Organics } \\
\hline $1,2,4$-Trichlorobenzene $(\mu \mathrm{g} / \mathrm{kg})$ & 12 & 10 & 10 & 10 \\
\hline 1,2-Dichlorobenzene $(\mu \mathrm{g} / \mathrm{kg})$ & 12 & 10 & 10 & 10 \\
\hline 1,3-Dichlorobenzene $(\mu \mathrm{g} / \mathrm{kg})$ & 12 & 10 & 10 & 10 \\
\hline 1,4-Dichlorobenzene $(\mu \mathrm{g} / \mathrm{kg})$ & 12 & 10 & 10 & 10 \\
\hline 2,4,6-Trichlorophenol $(\mu \mathrm{g} / \mathrm{kg})$. & 12 & 30 & 30 & 30 \\
\hline 2,4-Dichlorophenol ( $\mu \mathrm{g} / \mathrm{kg})$ & 12 & 30 & 30 & 30 \\
\hline 2,4-Dimethylphenol $(\mu \mathrm{g} / \mathrm{kg})$ & 12 & 30 & 30 & 30 \\
\hline 2,4-Dinitrophenol ( $\mu \mathrm{g} / \mathrm{kg})$ & 12 & 30 & 30 & 30 \\
\hline 2,4-Dinitrotoluene $(\mu \mathrm{g} / \mathrm{kg})$ & 12 & 10 & 10 & 10 \\
\hline 2,6-Dinitrotoluene $(\mu \mathrm{g} / \mathrm{kg})$ & 12 & 10 & 10 & 10 \\
\hline 2-Chloronaphthalene $(\mu \mathrm{g} / \mathrm{kg})$ & 12 & 10 & 10 & 10 \\
\hline 2-Chlorophenol $(\mu \mathrm{g} / \mathrm{kg})$ & 12 & 30 & 30 & 30 \\
\hline 2-Methyl-4,6-dinitrophenol $(\mu \mathrm{g} / \mathrm{kg})$ & 12 & 30 & 30 & 30 \\
\hline 2-Nitrophenol $(\mu \mathrm{g} / \mathrm{kg})$ & 12 & 30 & 30 & 30 \\
\hline 3,3'-Dichlorobenzidine $(\mu \mathrm{g} / \mathrm{kg})$ & 12 & 10 & 10 & 10 \\
\hline 4-Bromophenyl phenyl ether $(\mu \mathrm{g} / \mathrm{kg})$ & 12 & 10 . & 10 & 10 \\
\hline
\end{tabular}


Table A-2. (continued)

\begin{tabular}{|c|c|c|c|c|}
\hline Analysis (units) & $\begin{array}{l}\text { Number of } \\
\text { samples }\end{array}$ & $\begin{array}{c}\text { Minimum } \\
\text { detection } \\
\text { limit }\end{array}$ & $\begin{array}{c}\text { Average } \\
\text { detection } \\
\text { limit }\end{array}$ & $\begin{array}{c}\text { Maximum } \\
\text { detection } \\
\text { limit }\end{array}$ \\
\hline 4-Chloro-3-methylphenol ( $\mu \mathrm{g} / \mathrm{kg})$ & 12 & 30 & 30 & 30 \\
\hline 4-Chlorophenylphenyl ether $(\mu \mathrm{g} / \mathrm{kg})$ & 12 & 10 & 10 & 10 \\
\hline 4-Nitrophenol $(\mu \mathrm{g} / \mathrm{kg})$ & 12 & 30 & 30 & 30 \\
\hline Acenaphthene $(\mu \mathrm{g} / \mathrm{kg})$ & 12 & 10 & 10 & 10 \\
\hline Acenaphthylene $(\mu \mathrm{g} / \mathrm{kg})$ & 12 & 10 & 10 & 10 \\
\hline Anthracene $(\mu \mathrm{g} / \mathrm{kg})$ & 12 & 10 & 10 & 10 \\
\hline Benzidine $(\mu \mathrm{g} / \mathrm{kg})$ & 12 & 30 & 30 & 30 \\
\hline Benzo(a)anthracene $(\mu \mathrm{g} / \mathrm{kg})$ & 12 & 10 & 10 & 10 \\
\hline Benzo(a)pyrene $(\mu \mathrm{g} / \mathrm{kg})$ & 12 & 10 & 10 & 10 \\
\hline Benzo(b)fluoranthene $(\mu \mathrm{g} / \mathrm{kg})$ & 12 & 10 & 10 & 10 \\
\hline Benzo(ghi)perylene $(\mu \mathrm{g} / \mathrm{kg})$ & 12 & 10 & 10 & 10 \\
\hline Benzo(k)fluoranthene $(\mu \mathrm{g} / \mathrm{kg})$ & 12 & 10 & 10 & 10 \\
\hline Bis(2-chloroethoxy)methane $(\mu \mathrm{g} / \mathrm{kg})$ & 12 & 10 & 10 & 10 \\
\hline Bis(2-chloroethyl) ether $(\mu \mathrm{g} / \mathrm{kg})$ & 12 & 10 & 10 & 10 \\
\hline Butylbenzylphthalate $(\mu \mathrm{g} / \mathrm{kg})$ & 12 & 10 & 10 & 10 \\
\hline Chrysene $(\mu \mathrm{g} / \mathrm{kg})$ & 12 & 10 & 10 & 10 \\
\hline Di-n-butylphthalate $(\mu \mathrm{g} / \mathrm{kg})$ & 12 & 10 & 10 & 10 \\
\hline Dibenzo(a,h)anthracene $(\mu \mathrm{g} / \mathrm{kg})$ & 12 & 10 & 10 & 10 \\
\hline Diethylphthalate $(\mu \mathrm{g} / \mathrm{kg})$ & 12 & 10 & 10 & 10 \\
\hline Dimethylphthalate $(\mu \mathrm{g} / \mathrm{kg})$ & 12 & 10 & 10 & 10 \\
\hline Fluoranthene $(\mu \mathrm{g} / \mathrm{kg})$ & 12 & 10 & 10 & 10 \\
\hline Fluorene $(\mu \mathrm{g} / \mathrm{kg})$ & 12 & 10 & 10 & 10 \\
\hline Hexachlorobenzene $(\mu \mathrm{g} / \mathrm{kg})$ & 12 & 10 & 10 & 10 \\
\hline Hexachlorobutadiene $(\mu \mathrm{g} / \mathrm{kg})$ & 12 & 10 & 10 & 10 \\
\hline Hexachlorocyclopentadiene $(\mu \mathrm{g} / \mathrm{kg})$ & 12 & 10 & 10 & 10 \\
\hline Hexachloroethane $(\mu \mathrm{g} / \mathrm{kg})$ & 12 & 10 & 10 & 10 \\
\hline Indeno(1,2,3-cd)pyrene $(\mu \mathrm{g} / \mathrm{kg})$ & 12 & 10 & 10 & 10 \\
\hline Isophorone $(\mu \mathrm{g} / \mathrm{kg})$ & 12 & 10 & 10 & 10 \\
\hline N-Nitroso-di-n-propylamine $(\mu \mathrm{g} / \mathrm{kg})$ & 12 & 10 & 10 & 10 \\
\hline
\end{tabular}


Table $A-2$. (continued)

\begin{tabular}{|c|c|c|c|c|}
\hline Analysis (units) & $\begin{array}{l}\text { Number of } \\
\text { samples }\end{array}$ & $\begin{array}{l}\text { Minimum } \\
\text { detection } \\
\text { limit }\end{array}$ & $\begin{array}{c}\text { Average } \\
\text { detection } \\
\text { limit }\end{array}$ & $\begin{array}{c}\text { Maximum } \\
\text { detection } \\
\text { limit }\end{array}$ \\
\hline $\mathrm{N}$-Nitrosodimethylamine $(\mu \mathrm{g} / \mathrm{kg})$ & 12 & 10 & 10 & 10 \\
\hline N-Nitrosodiphenylamine $(\mu \mathrm{g} / \mathrm{kg})$ & 12 & 10 & 10 & 10 \\
\hline Naphthalene $(\mu \mathrm{g} / \mathrm{kg})$ & 12 & 10 & 10 & 10 \\
\hline Nitrobenzene $(\mu \mathrm{g} / \mathrm{kg})$ & 12 & 10 & 10 & 10 \\
\hline Pentachlorophenol $(\mu \mathrm{g} / \mathrm{kg})$ & 12 & 30 & 30 & 30 \\
\hline Phenanthrene $(\mu \mathrm{g} / \mathrm{kg})$ & 12 & 10 & 10 & 10 \\
\hline Phenol ( $\mu \mathrm{g} / \mathrm{kg})$ & 12 & 30 & 30 & 30 \\
\hline Pyrene $(\mu \mathrm{g} / \mathrm{kg})$ & 12 & 10 & 10 & 10 \\
\hline bis(2-Chloroisopropyl)ether $(\mu \mathrm{g} / \mathrm{kg})$ & 12 & 10 & 10 & 10 \\
\hline \multicolumn{5}{|l|}{ Volatile Organics } \\
\hline 1,1,1-Trichloroethane $(\mu \mathrm{g} / \mathrm{kg})$ & 12 & 10 & 10 & 10 \\
\hline 1,1,2,2-Tetrachloroethane $(\mu \mathrm{g} / \mathrm{kg})$ & 12 & 10 & 10 & 10 \\
\hline 1,1,2-Trichloroethane $(\mu \mathrm{g} / \mathrm{kg})$ & 12 & 10 & 10 & 10 \\
\hline 1,1-Dichloroethane $(\mu \mathrm{g} / \mathrm{kg})$ & 12 & 10 & 10 & 10 \\
\hline 1,1-Dichloroethene $(\mu \mathrm{g} / \mathrm{kg})$ & 12 & 10 & 10 & 10 \\
\hline 1,2-Dichloroethane $(\mu \mathrm{g} / \mathrm{kg})$ & 12 & 10 & 10 & 10 \\
\hline 1,2-Dichloropropane ( $\mu \mathrm{g} / \mathrm{kg})$ & 12 & 10 & 10 & 10 \\
\hline 2-Butanone $(\mu \mathrm{g} / \mathrm{kg})$ & 12 & 10 & 10 & 10 \\
\hline 2-Chloroethylvinyl ether $(\mu \mathrm{g} / \mathrm{kg})$ & 12 & 10 & 10 & 10 \\
\hline 2-Hexanone $(\mu \mathrm{g} / \mathrm{kg})$ & 12 & 10 & 10 & 10 \\
\hline 4-Methyl-2-pentanone $(\mu \mathrm{g} / \mathrm{kg})$ & 12 & 10 & 10 & 10 \\
\hline Acetone $(\mu \mathrm{g} / \mathrm{kg})$ & 12 & 100 & 100 & 100 \\
\hline Benzene $(\mu \mathrm{g} / \mathrm{kg})$ & 12 & 10 & 10 & 10 \\
\hline Bromodichloromethane $(\mu \mathrm{g} / \mathrm{kg})$ & 12 & 10 & 10 & 10 \\
\hline Bromoform $(\mu \mathrm{g} / \mathrm{kg})$ & 12 & 10 & 10 & 10 \\
\hline Bromomethane $(\mu \mathrm{g} / \mathrm{kg})$ & 12 & 10 & 10 & 10 \\
\hline Carbon disulfide $(\mu \mathrm{g} / \mathrm{kg})$ & 12 & 10 & 10 & 10 \\
\hline Carbon tetrachloride $(\mu \mathrm{g} / \mathrm{kg})$ & 12 & 10 & 10 & 10 \\
\hline
\end{tabular}


Table A-2. (continued)

\begin{tabular}{lcccc}
\hline \multicolumn{1}{c}{ Analysis (units) } & $\begin{array}{c}\text { Number of } \\
\text { samples }\end{array}$ & $\begin{array}{c}\text { Minimum } \\
\text { detection } \\
\text { limit }\end{array}$ & $\begin{array}{c}\text { Average } \\
\text { detection } \\
\text { limit }\end{array}$ & $\begin{array}{c}\text { Maximum } \\
\text { detection } \\
\text { limit }\end{array}$ \\
\hline Chlorobenzene $(\mu \mathrm{g} / \mathrm{kg})$ & 12 & 10 & 10 & 10 \\
Chloroethane $(\mu \mathrm{g} / \mathrm{kg})$ & 12 & 10 & 10 & 10 \\
Chloroform $(\mu \mathrm{g} / \mathrm{kg})$ & 12 & 10 & 10 & 10 \\
Chloromethane $(\mu \mathrm{g} / \mathrm{kg})$ & 12 & 10 & 10 & 10 \\
Dibromochloromethane $(\mu \mathrm{g} / \mathrm{kg})$ & 12 & 10 & 10 & 10 \\
Dimethylbenzene $(\mu \mathrm{g} / \mathrm{kg})$ & 12 & 10 & 10 & 10 \\
Ethylbenzene $(\mu \mathrm{g} / \mathrm{kg})$ & 12 & 10 & 10 & 10 \\
Styrene $(\mu \mathrm{g} / \mathrm{kg})$ & 12 & 10 & 10 & 10 \\
Toluene $(\mu \mathrm{g} / \mathrm{kg})$ & 12 & 10 & 10 & 10 \\
Trichlorofluoromethane $(\mu \mathrm{g} / \mathrm{kg})$ & 12 & 10 & 10 & 10 \\
Vinyl chloride $(\mu \mathrm{g} / \mathrm{kg})$ & 12 & 10 & 10 & 10 \\
cis-1,2-Dichloroethene $(\mu \mathrm{g} / \mathrm{kg})$ & 12 & 10 & 10 & 10 \\
cis-1,3-Dichloropropene $(\mu \mathrm{g} / \mathrm{kg})$ & 12 & 10 & 10 & 10 \\
trans-1,2-Dichloroethene $(\mu \mathrm{g} / \mathrm{kg})$ & 12 & 10 & 10 & 10 \\
trans-1,3-Dichloropropene $(\mu \mathrm{g} / \mathrm{kg})$ & 12 & 10 & 10 & 10 \\
\hline & & & & \\
& & & 10 & 10 \\
\hline
\end{tabular}


Table A-3. Kerr Hollow Quarry sediment samples taken from 1990 to 1992

(Summary of chemical concentrations with at least one result greater than the detection limit)

\begin{tabular}{|c|c|c|c|c|c|c|c|}
\hline Analysis (units) & $\begin{array}{l}\text { Proportion } \\
\text { >detection } \\
\quad \text { limit }\end{array}$ & $\begin{array}{c}\text { Minimum } \\
\text { detected } \\
\text { concentration }\end{array}$ & $\begin{array}{c}\text { Maximum } \\
\text { detected } \\
\text { concentration }\end{array}$ & Mean $^{2}$ & $\begin{array}{l}\text { Standard } \\
\text { deviation }\end{array}$ & $\begin{array}{l}\text { Detects } \\
\text { > reference }\end{array}$ & $\begin{array}{l}\text { Reference } \\
\text { criteria }^{\mathrm{b}}\end{array}$ \\
\hline \multicolumn{8}{|l|}{ Physical Parameters } \\
\hline $\begin{array}{l}\text { Percent Moisture } \\
\text { (\%) }\end{array}$ & $14 / 14$ & 54 & 84 & 68.07 & 10.19 & & . \\
\hline \multicolumn{8}{|l|}{ Metals } \\
\hline $\begin{array}{l}\text { Cadmium, AA } \\
(\mathrm{mg} / \mathrm{kg})\end{array}$ & 212 & 0.98 & 1.8 & 1.39 & 0.5798 & 212 & 0.13 \\
\hline $\begin{array}{l}\text { Chromium, AA } \\
\text { (mg/kg) }\end{array}$ & $2 / 2$ & 12 & 13 & 12.5 & 0.7071 & $0 / 2$ & 53 \\
\hline Lead, AA (mg/kg) & $18 / 18$ & 11 & 44 & 21.72 & 8.358 & $0 / 18$ & 57 \\
\hline Mercury (mg/kg) & $18 / 18$ & 1.1 & 34 & 13.32 & 11.55 & $18 / 18$ & 0.38 \\
\hline Aluminum (mg/kg) & $18 / 18$ & 3210 & 15200 & 8104 & 3994 & $0 / 18$ & 42035 \\
\hline Barium (mg/kg) & $18 / 18$ & 32.3 & 115 & 66.22 & 24.64 & $0 / 18$ & 147 \\
\hline Beryllium (mg/kg) & $18 / 18$ & 0.3 & 1.5 & 0.7167 & 0.3148 & $0 / 18$ & 2 \\
\hline Boron (mg/kg) & $18 / 18$ & 6 & 23 & 11.61 & 4.06 & $0 / 18$ & 27 \\
\hline Cadmium (mg/kg) & $1 / 18$ & 2 & 2 & 1.389 & 0.2687 & $1 / 18$ & 0.13 \\
\hline Calcium (mg/kg) & $18 / 18$ & 3420 & 32600 & 17420 & 8101 & $18 / 18$ & 3051 \\
\hline Cerium (mg/kg) & $12 / 18$ & 11 & 50 & 19.38 & 14.37 & & - \\
\hline Chromium (mg/kg) & $18 / 18$ & 7 & 23 & 14 & 4.615 & $0 / 18$ & 53 \\
\hline Cobalt (mg/kg) & $18 / 18$ & 2 & 7 & 3.778 & 1.555 & $0 / 18$ & 30 \\
\hline Copper (mg/kg) & $18 / 18$ & 20 & 1110 & 301.8 & 326.7 & $15 / 18$ & 35 \\
\hline Iron (mg/kg) & $18 / 18$ & 3120 & 15500 & 6899 & 3268 & $0 / 18$ & 57774 \\
\hline Lead (mg/kg) & $12 / 18$ & 10 & 40 & 18.28 & 11.1 & $0 / 18$ & 57 \\
\hline Lithium (mg/kg) & $17 / 18$ & 10 & 52 & 29.78 & 13.73 & $7 / 18$ & 36 \\
\hline Magnesium (mg/kg) & $18 / 18$ & 890 & 8380 & 3416 & 2201 & $6 / 18$ & 4258 \\
\hline Manganese (mg/kg) & $18 / 18$ & 27 & 206 & 102.5 & 53.05 & $0 / 18$ & 1998 \\
\hline Nickel (mg/kg) & $18 / 18$ & 7 & 29 & 15.22 & 7.281 & $0 / 18$ & 35 \\
\hline Niobium (mg/kg) & $13 / 18$ & 6 & 55 & 12.16 & 12.76 & & . \\
\hline Phosphorus (mg/kg) & $18 / 18$ & 80 & 250 & 142.8 & 49.09 & & - \\
\hline Potassium (mg/kg) & $17 / 18$ & 300 & 3300 & 1288 & 944.7 & $0 / 18$ & 4891 \\
\hline Scandium (mg/kg) & $12 / 12$ & 0.7 & 2.6 & 1.35 & 0.5317 & & - \\
\hline Sodium (mg/kg) & $9 / 18$ & 30 & 70 & 27.25 & 17.44 & $0 / 18$ & 502 \\
\hline Strontium $(\mathrm{mg} / \mathrm{kg})$ & $18 / 18$ & 3.9 & 30.9 & 17.73 & 7.128 & $4 / 18$ & 22 \\
\hline
\end{tabular}

95-044P/051995 
Table A-3 (continued)

\begin{tabular}{|c|c|c|c|c|c|c|c|}
\hline Analysis (units) & $\begin{array}{l}\text { Proportion } \\
\text { >detection } \\
\text { limit }\end{array}$ & $\begin{array}{l}\text { Minimum } \\
\text { detected } \\
\text { concentration }\end{array}$ & $\begin{array}{c}\text { Maximum } \\
\text { detected } \\
\text { concentration }\end{array}$ & Mean $^{2}$ & $\begin{array}{l}\text { Standard } \\
\text { deviation }\end{array}$ & $\begin{array}{c}\text { Detects } \\
>\text { reference }\end{array}$ & $\begin{array}{c}\text { Reference } \\
\text { criteriab }\end{array}$ \\
\hline Titanium (mg/kg) & $18 / 18$ & 35 & 110 & 72.17 & 23.22 & & . \\
\hline Vanadium (mg/kg) & $18 / 18$ & 7 & 36 & 13 & 7.178 & $0 / 18$ & 75 \\
\hline Yttrium (mg/kg) & $10 / 10$ & 73 & 142 & 99.7 & 21.27 & & . \\
\hline Zinc (mg/kg) & 818 & 103 & 354 & 174.9 & 78.13 & $4 !$ & 160 \\
\hline Zirconium (mg/kg) & $18 / 18$ & 9 & 131 & 55.83 & 39.81 & & . \\
\hline \multicolumn{8}{|c|}{ Semivolatile Orgamics } \\
\hline $\begin{array}{l}\text { Bis(2-ethylhexyl) } \\
\text { phthalate }(\mu \mathrm{g} / \mathrm{kg})\end{array}$ & $2 / 12$ & 1800 & 2700 & 379.2 & 894.7 & & - \\
\hline $\begin{array}{l}\text { Di-n-octylphthalate } \\
(\mu \mathrm{g} / \mathrm{kg})\end{array}$ & $2 / 12$ & 1600 & 2500 & 345.8 & 818.8 & & . \\
\hline \multicolumn{8}{|l|}{ Volatile Organics } \\
\hline $\begin{array}{l}\text { Methylene chloride } \\
(\mu \mathrm{g} / \mathrm{kg})\end{array}$ & $4 / 12$ & 16 & 19 & 10.04 & 6.391 & & . \\
\hline $\begin{array}{l}\text { Tetrachloroethene } \\
(\mu \mathrm{g} / \mathrm{kg})\end{array}$ & $1 / 12$ & 330 & 330 & 32.08 & 93.82 & & - \\
\hline $\begin{array}{l}\text { Trichloroethene } \\
(\mu \mathrm{g} / \mathrm{kg})\end{array}$ & $1 / 12$ & 13 & 13 & 5.667 & 2.309 & & - \\
\hline
\end{tabular}

a Non-detects set to $1 / 2$ the reported detection limit.

${ }^{b}$ Reference concentration is the smaller of the maximum or $95 \%$ upper tolerance limit concentration of soil samples from the Background Soil Characterization Project at the Oak Ridge Reservation.

Output of program kerrsd01 on 08MAR94 at 07:35 using dataset paramtr. 
Table A-4. Kerr Hollow Quarry sediment samples taken from 1990 to 1992 (Summary of radionuclide concentrations)

\begin{tabular}{|c|c|c|c|c|c|c|c|c|c|}
\hline $\begin{array}{l}\text { Analysis } \\
\text { (units) }\end{array}$ & $\begin{array}{l}\text { Proportion } \\
>\text { detection } \\
\text { limit }^{a}\end{array}$ & $\begin{array}{c}\text { Minimum } \\
\text { detected } \\
\text { concentration }\end{array}$ & $\begin{array}{c}\text { Maximum } \\
\text { detected } \\
\text { concentration }\end{array}$ & Mean & $\begin{array}{l}\text { Standard } \\
\text { deviation }\end{array}$ & $\begin{array}{l}\text { Standard } \\
\text { error of } \\
\text { the mean }\end{array}$ & $\begin{array}{l}\text { Counting } \\
\text { uncertainty }\end{array}$ & $\begin{array}{l}\text { No. of Resuits } \\
>\text { reference } \\
\text { concentration }\end{array}$ & $\begin{array}{c}\text { Reference } \\
\text { concentration }\end{array}$ \\
\hline $\begin{array}{l}\text { Beta activity } \\
\text { (pCi/g) }\end{array}$ & $18 / 18$ & 6.7 & 263 & 34.74 & 58.58 & 13.81 & 0.3233 & & . \\
\hline $\begin{array}{l}\text { Gamma } \\
\text { activity } \\
\text { (pCi/g) }\end{array}$ & $16 / 16$ & 6.9 & 29 & 15.48 & 5.598 & 1.399 & 0.5287 & & . \\
\hline $\begin{array}{l}\text { Gross Alpha } \\
\text { (pCi/g) }\end{array}$ & $18 / 18$ & 5.6 & 92 & 29.33 & 29.59 & 6.974 & 0.5135 & & . \\
\hline $\begin{array}{l}\text { Uranium } \\
(\mathrm{mg} / \mathrm{kg})\end{array}$ & $18 / 18$ & 3.37 & 336 & 42.3 & 78.99 & 18.62 & 0.1722 & & . \\
\hline $\begin{array}{l}\text { Uranium-234 } \\
\text { (pCi/g) }\end{array}$ & $6 / 6$ & 7.4 & 41 & 19.93 & 13.34 & 5.447 & 0.7902 & & . \\
\hline $\begin{array}{l}\text { Uranium-235 } \\
\text { (\%) }\end{array}$ & $18 / 18$ & 0.29 & 1.16 & 0.6806 & 0.249 & 0.05869 & . & & . \\
\hline $\begin{array}{l}\text { Uranium-235 } \\
\text { (pCi/g) }\end{array}$ & $6 / 7$ & 0 & 1.6 & 0.78 & 0.6183 & 0.2337 & 0.04657 & $3 /$ & 1 \\
\hline $\begin{array}{l}\text { Uranium-238 } \\
\text { (pCi/g) }\end{array}$ & $6 / 6$ & 6.9 & 32 & 16.95 & 11.75 & 4.799 & 0.7133 & 61 & 2 \\
\hline
\end{tabular}

${ }^{a}$ Radionuclide results less than the counting uncertainty were considered nondetects.

${ }^{b}$ The 1 sigma pooled counting uncertainty was calculated as the square root of the sum of the squared counting errors divided by 2 times the number of observations.

${ }^{c}$ Reference concentration is the smaller of the maximum or $95 \%$ upper tolerance limit concentration of soil samples from the Background Soil Characterization Project at the Oak Ridge Reservation.

Output of program kerrsd01 on 08MAR94 at 07:35 using dataset paramtr. 
Table A-5. Kerr Hollow Quarry sediment samples taken from 1990 to 1992 (Analytes with TCLP results less than detection limit)

\begin{tabular}{|c|c|c|c|c|}
\hline Analysis (units) & $\begin{array}{c}\text { Number of } \\
\text { samples }\end{array}$ & $\begin{array}{c}\text { Minimum } \\
\text { detection } \\
\text { limit }\end{array}$ & $\begin{array}{c}\text { Average } \\
\text { detection } \\
\text { limit }\end{array}$ & $\begin{array}{c}\text { Maximum } \\
\text { detection } \\
\text { limit }\end{array}$ \\
\hline \multicolumn{5}{|l|}{ TCLP Herbicides and Pesticides } \\
\hline $2,4-\mathrm{D}(\mu \mathrm{g} / \mathrm{L})$ & 2 & 1000 & 1000 & 1000 \\
\hline Chlordane $(\mu \mathrm{g} / \mathrm{L})$ & 2 & 3 & 3 & 3 \\
\hline Endrin $(\mu \mathrm{g} / \mathrm{L})$ & 12 & 2 & 8.67 & 10 \\
\hline Heptachlor $(\mu \mathrm{g} / \mathrm{L})$ & 2 & 0.8 & 0.8 & 0.8 \\
\hline Heptachlor epoxide $(\mu \mathrm{g} / \mathrm{L})$ & 2 & 0.8 & 0.8 & 0.8 \\
\hline Methoxychlor $(\mu \mathrm{g} / \mathrm{L})$ & 12 & 10 & 175 & 1000 \\
\hline Silvex $(\mu \mathrm{g} / \mathrm{L})$ & 2 & 100 & 100 & 100 \\
\hline Toxaphene $(\mu \mathrm{g} / \mathrm{L})$ & 12 & 10 & 16.7 & 50 \\
\hline gamma-BHC $(\mu \mathrm{g} / \mathrm{L})$ & 12 & 10 & 15 & 40 \\
\hline \multicolumn{5}{|l|}{ TCLP Metals } \\
\hline Arsenic $(\mathrm{mg} / \mathrm{L})$ & 18 & 0.5 & 0.5 & 0.5 \\
\hline Chromium (mg/L) & 18 & 0.5 & 0.5 & 0.5 \\
\hline Selenium $(\mathrm{mg} / \mathrm{L})$ & 18 & 0.1 & 0.1 & 0.1 \\
\hline Silver $(\mathrm{mg} / \mathrm{L})$ & 18 & 0.5 & 0.5 & 0.5 \\
\hline \multicolumn{5}{|l|}{ TCLP Semivolatile Organics } \\
\hline 1,2,4-Trichlorobenzene $(\mu \mathrm{g} / \mathrm{L})$ & 10 & 10 & 10 & 10 \\
\hline 1,2-Dichlorobenzene $(\mu \mathrm{g} / \mathrm{L})$ & 10 & 10 & 10 & 10 \\
\hline 1,3-Dichlorobenzene $(\mu \mathrm{g} / \mathrm{L})$ & 10 & 10 & 10 & 10 \\
\hline 1,4-Dichlorobenzene $(\mu \mathrm{g} / \mathrm{L})$ & 10 & 10 & 10 & 10 \\
\hline 2,4,5-Trichlorophenol $(\mu \mathrm{g} / \mathrm{L})$ & 6 & 40000 & 40000 & 40000 \\
\hline 2,4,6-Trichlorophenol $(\mu \mathrm{g} / \mathrm{L})$ & 16 & 30 & 93.8 & 200 \\
\hline 2,4-Dichlorophenol $(\mu \mathrm{g} / \mathrm{L})$ & 10 & 30 & 30 & 30 \\
\hline 2,4-Dimethylphenol $(\mu \mathrm{g} / \mathrm{L})$ & 10 & 30 & 30 & 30 \\
\hline 2,4-Dinitrophenol $(\mu \mathrm{g} / \mathrm{L})$ & 10 & 30 & 30 & 30 \\
\hline 2,4-Dinitrotoluene $(\mu \mathrm{g} / \mathrm{L})$ & 16 & 10 & 11.1 & 13 \\
\hline 2,6-Dinitrotoluene $(\mu \mathrm{g} / \mathrm{L})$ & 10 & 10 & 10 & 10 \\
\hline 2-Chloronaphthalene $(\mu \mathrm{g} / \mathrm{L})$ & 10 & 10 & 10 & 10 \\
\hline 2-Chlorophenol $(\mu \mathrm{g} / \mathrm{L})$ & 10 & 30 & 30 & 30 \\
\hline
\end{tabular}


Table A-5 (continued)

\begin{tabular}{|c|c|c|c|c|}
\hline Analysis (units) & $\begin{array}{l}\text { Number of } \\
\text { samples }\end{array}$ & $\begin{array}{c}\text { Minimum } \\
\text { detection } \\
\text { limit }\end{array}$ & $\begin{array}{c}\text { Average } \\
\text { detection } \\
\text { limit }\end{array}$ & $\begin{array}{l}\text { Maximum } \\
\text { detection } \\
\text { limit }\end{array}$ \\
\hline 2-Methyl-4,6-dinitrophenol $(\mu \mathrm{g} / \mathrm{L})$ & 10 & 30 & 30 & 30 \\
\hline 2-Methylphenol $(\mu \mathrm{g} / \mathrm{L})$ & 6 & 20000 & 20000 & 20000 \\
\hline 2-Nitrophenol $(\mu \mathrm{g} / \mathrm{L})$ & 10 & 30 & 30 & 30 \\
\hline 3,3'-Dichlorobenzidine $(\mu \mathrm{g} / \mathrm{L})$ & 10 & 10 & 10 & 10 \\
\hline 3-and/or 4-Methylphenol $(\mu \mathrm{g} / \mathrm{L})$ & 6 & 40000 & 40000 & 40000 \\
\hline 4-Bromophenyl phenyl ether $(\mu \mathrm{g} / \mathrm{L})$ & 10 & 10 & 10 & 10 \\
\hline 4-Chloro-3-methylphenol ( $\mu \mathrm{g} / \mathrm{L})$ & 10 & 30 & 30 & 30 \\
\hline 4-Chlorophenylphenyl ether $(\mu \mathrm{g} / \mathrm{L})$ & 10 & 10 & 10 & 10 \\
\hline 4-Nitrophenol $(\mu \mathrm{g} / \mathrm{L})$ & 10 & 30 & 30 & 30 \\
\hline Acenaphthene $(\mu \mathrm{g} / \mathrm{L})$ & 10 & 10 & 10 & 10 \\
\hline Acenaphthylene $(\mu \mathrm{g} / \mathrm{L})$ & 10 & 10 & 10 & 10 \\
\hline Anthracene $(\mu \mathrm{g} / \mathrm{L})$ & 10 & 10 & 10 & 10 \\
\hline Benzidine $(\mu \mathrm{g} / \mathrm{L})$ & 10 & 30 & 30 & 30 \\
\hline Benzo(a)anthracene $(\mu \mathrm{g} / \mathrm{L})$ & 10 & 10 & 10 & 10 \\
\hline Benzo(a)pyrene $(\mu \mathrm{g} / \mathrm{L})$ & 10 & 10 & 10 & 10 \\
\hline Benzo(b)fluoranthene $(\mu \mathrm{g} / \mathrm{L})$ & 10 & 10 & 10 & 10 \\
\hline Benzo(ghi)perylene $(\mu \mathrm{g} / \mathrm{L})$ & 10 & 10 & 10 & 10 \\
\hline Benzo(k)fluoranthene $(\mu \mathrm{g} / \mathrm{L})$ & 10 & 10 & 10 & 10 \\
\hline Bis(2-chloroethoxy)methane $(\mu \mathrm{g} / \mathrm{L})$ & 10 & 10 & 10 & 10 \\
\hline Bis(2-chloroethyl) ether $(\mu \mathrm{g} / \mathrm{L})$ & 10 & 10 & 10 & 10 \\
\hline Bis(2-ethylhexyl)phthalate $(\mu \mathrm{g} / \mathrm{L})$ & 10 & 10 & 10 & 10 \\
\hline Butylbenzylphthalate $(\mu \mathrm{g} / \mathrm{L})$ & 10 & 10 & 10 & 10 \\
\hline Chrysene $(\mu \mathrm{g} / \mathrm{L})$ & 10 & 10 & 10 & 10 \\
\hline Di-n-butylphthalate $(\mu \mathrm{g} / \mathrm{L})$ & 10 & 10 & 10 & 10 \\
\hline Di-n-octylphthalate $(\mu \mathrm{g} / \mathrm{L})$ & 10 & 10 & 10 & 10 \\
\hline Dibenzo(a,h)anthracene $(\mu \mathrm{g} / \mathrm{L})$ & 10 & 10 & 10 & 10 \\
\hline Diethylphthalate $(\mu \mathrm{g} / \mathrm{L})$ & 10 & 10 & 10 & 10 \\
\hline Dimethylphthalate $(\mu \mathrm{g} / \mathrm{L})$ & 10 & 10 & 10 & 10 \\
\hline Fluoranthene $(\mu \mathrm{g} / \mathrm{L})$ & 10 & 10 & 10 & 10 \\
\hline Fluorene $(\mu \mathrm{g} / \mathrm{L})$ & 10 & 10 & 10 & 10 \\
\hline Hexachlorobenzene $(\mu \mathrm{g} / \mathrm{L})$ & 16 & 10 & 11.1 & 13 \\
\hline
\end{tabular}


Table A-5 (continued)

\begin{tabular}{|c|c|c|c|c|}
\hline Analysis (units) & $\begin{array}{c}\text { Number of } \\
\text { samples }\end{array}$ & $\begin{array}{l}\text { Minimum } \\
\text { detection } \\
\text { limit }\end{array}$ & $\begin{array}{c}\text { Average } \\
\text { detection } \\
\text { limit }\end{array}$ & $\begin{array}{c}\text { Maximum } \\
\text { detection } \\
\text { limit }\end{array}$ \\
\hline Hexachlorobutadiene $(\mu \mathrm{g} / \mathrm{L})$ & 16 & 10 & 25 & 50 \\
\hline Hexachlorocyclopentadiene $(\mu \mathrm{g} / \mathrm{L})$ & 10 & 10 & 10 & 10 \\
\hline Hexachloroethane $(\mu \mathrm{g} / \mathrm{L})$ & 16 & 10 & 119 & 300 \\
\hline Indeno(1,2,3-cd)pyrene $(\mu \mathrm{g} / \mathrm{L})$ & 10 & 10 & 10 & 10 \\
\hline Isophorone $(\mu \mathrm{g} / \mathrm{L})$ & 10 & 10 & 10 & 10 \\
\hline N-Nitroso-di-n-propylamine $(\mu \mathrm{g} / \mathrm{L})$ & 10 & 10 & 10 & 10 \\
\hline N-Nitrosodimethylamine $(\mu \mathrm{g} / \mathrm{L})$ & 10 & 10 & 10 & 10 \\
\hline N-Nitrosodiphenylamine $(\mu \mathrm{g} / \mathrm{L})$ & 10 & 10 & 10 & 10 \\
\hline Naphthalene $(\mu \mathrm{g} / \mathrm{L})$ & 10 & 10 & 10 & 10 \\
\hline Nitrobenzene $(\mu \mathrm{g} / \mathrm{L})$ & 16 & 10 & 81.3 & 200 \\
\hline Pentachlorophenol $(\mu \mathrm{g} / \mathrm{L})$ & 16 & 30 & 3770 & 10000 \\
\hline Phenanthrene $(\mu \mathrm{g} / \mathrm{L})$ & 10 & 10 & 10 & 10 \\
\hline Phenol $(\mu \mathrm{g} / \mathrm{L})$ & 10 & 30 & 30 & 30 \\
\hline Pyrene $(\mu \mathrm{g} / \mathrm{L})$ & 10 & 10 & 10 & 10 \\
\hline Pyridine $(\mu \mathrm{g} / \mathrm{L})$ & 6 & 500 & 500 & 500 \\
\hline bis(2-Chloroisopropyl)ether ( $\mu \mathrm{g} / \mathrm{L})$ & 10 & 10 & 10 & 10 \\
\hline \multicolumn{5}{|l|}{ TCLP Volatile Organics } \\
\hline 1,1,1-Trichloroethane $(\mu \mathrm{g} / \mathrm{L})$ & 10 & 10 & 10 & 10 \\
\hline 1,1,2,2-Tetrachloroethane $(\mu \mathrm{g} / \mathrm{L})$ & 10 & 10 & 10 & 10 \\
\hline 1,1,2-Trichloro-1,2,2-trifluoroethane $(\mu \mathrm{g} / \mathrm{L})$ & 10 & 10 & 10 & 10 \\
\hline 1,1,2-Trichloroethane $(\mu \mathrm{g} / \mathrm{L})$ & 10 & 10 & 10 & 10 \\
\hline 1,1-Dichloroethane $(\mu \mathrm{g} / \mathrm{L})$ & 10 & 10 & 10 & 10 \\
\hline 1,1-Dichloroethene $(\mu \mathrm{g} / \mathrm{L})$ & 18 & 10 & 36.7 & 70 \\
\hline 1,2-Dichloroethane $(\mu \mathrm{g} / \mathrm{L})$ & 18 & 10 & 228 & 500 \\
\hline 1,2-Dichloropropane $(\mu \mathrm{g} / \mathrm{L})$ & 10 & 10 & 10 & 10 \\
\hline 1,4-Dichlorobenzene $(\mu \mathrm{g} / \mathrm{L})$ & 8 & 750 & 750 & 750 \\
\hline 2-Butanone $(\mu \mathrm{g} / \mathrm{L})$ & 18 & 10 & 8890 & 20000 \\
\hline 2-Chloroethylvinyl ether $(\mu \mathrm{g} / \mathrm{L})$ & 10 & 10 & 10 & 10 \\
\hline 2-Hexanone $(\mu \mathrm{g} / \mathrm{L})$ & 10 & 10 & 10 & 10 \\
\hline 4-Methyl-2-pentanone ( $\mu \mathrm{g} / \mathrm{L})$ & 10 & 10 & 10 & 10 \\
\hline
\end{tabular}


Table A-5 (continued)

\begin{tabular}{|c|c|c|c|c|}
\hline Analysis (units) & $\begin{array}{l}\text { Number of } \\
\text { samples }\end{array}$ & $\begin{array}{c}\text { Minimum } \\
\text { detection } \\
\text { limit }\end{array}$ & $\begin{array}{c}\text { Average } \\
\text { detection } \\
\text { limit }\end{array}$ & $\begin{array}{c}\text { Maximum } \\
\text { detection } \\
\text { limit }\end{array}$ \\
\hline Acetone $(\mu \mathrm{g} / \mathrm{L})$ & 10 & 100 & 100 & 100 \\
\hline Benzene $(\mu \mathrm{g} / \mathrm{L})$ & 18 & 10 & 27.8 & 50 \\
\hline Bromodichloromethane $(\mu \mathrm{g} / \mathrm{L})$ & 10 & 10 & 10 & 10 \\
\hline Bromoform $(\mu \mathrm{g} / \mathrm{L})$ & 10 & 10 & 10 & 10 \\
\hline Bromomethane $(\mu \mathrm{g} / \mathrm{L})$ & 10 & 10 & 10 & 10 \\
\hline Carbon disulfide $(\mu \mathrm{g} / \mathrm{L})$ & 10 & 10 & 10 & 10 \\
\hline Carbon tetrachloride $(\mu \mathrm{g} / \mathrm{L})$ & 18 & 10 & 27.8 & 50 \\
\hline Chlorobenzene $(\mu \mathrm{g} / \mathrm{L})$ & 18 & 10 & 4450 & 10000 \\
\hline Chloroethane $(\mu \mathrm{g} / \mathrm{L})$ & 10 & 10 & 10 & 10 \\
\hline Chloroform $(\mu \mathrm{g} / \mathrm{L})$ & 18 & 10 & 92.2 & 600 \\
\hline Chloromethane $(\mu \mathrm{g} / \mathrm{L})$ & 10 & 10 & 10 & 10 \\
\hline Dibromochloromethane $(\mu \mathrm{g} / \mathrm{L})$ & 10 & 10 & 10 & 10 \\
\hline Dimethylbenzene $(\mu \mathrm{g} / \mathrm{L})$ & 10 & 10 & 10 & 10 \\
\hline Ethylbenzene $(\mu \mathrm{g} / \mathrm{L})$ & 10 & 10 & 10 & 10 \\
\hline Methylene chloride $(\mu \mathrm{g} / \mathrm{L})$ & 10 & 10 & 10.9 & 19 \\
\hline Styrene $(\mu \mathrm{g} / \mathrm{L})$ & 10 & 10 & 10 & 10 \\
\hline Toluene $(\mu \mathrm{g} / \mathrm{L})$ & 10 & 10 & 10 & 10 \\
\hline Trichloroethene $(\mu \mathrm{g} / \mathrm{L})$ & 18 & 10 & 27.8 & 50 \\
\hline Trichlorofluoromethane $(\mu \mathrm{g} / \mathrm{L})$ & 10 & 10 & 10 & 10 \\
\hline Vinyl chloride $(\mu \mathrm{g} / \mathrm{L})$ & 18 & 10 & 14.4 & 20 \\
\hline cis-1,2-Dichloroethene $(\mu \mathrm{g} / \mathrm{L})$ & 10 & 10 & 10 & 10 \\
\hline cis-1,3-Dichloropropene $(\mu \mathrm{g} / \mathrm{L})$ & 10 & 10 & 10 & 10 \\
\hline trans-1,2-Dichloroethene $(\mu \mathrm{g} / \mathrm{L})$ & 10 & 10 & 10 & 10 \\
\hline
\end{tabular}


Table A-6. Kerr Hollow Quarry sediment samples taken from 19901992

(Analytes with at least one TCLP result greater than the detection limit)

\begin{tabular}{lccccccc}
\hline & $\begin{array}{c}\text { Proportion } \\
\text { > detection } \\
\text { limit }\end{array}$ & $\begin{array}{c}\text { Minimum } \\
\text { detected } \\
\text { concentration }\end{array}$ & $\begin{array}{c}\text { Maximum } \\
\text { detected } \\
\text { concentration }\end{array}$ & Mean & $\begin{array}{c}\text { Standard } \\
\text { deviation }\end{array}$ & $\begin{array}{c}\text { Detects } \\
\text { >TCLP } \\
\text { criteria }\end{array}$ & $\begin{array}{c}\text { TCLP } \\
\text { criteria }\end{array}$ \\
\hline Barium (mg/L) & $1 / 18$ & 11.8 & 11.8 & 5.378 & 1.603 & $0 / 18$ & 100 \\
Cadmium (mg/L) & $1 / 18$ & 0.13 & 0.13 & 0.05444 & 0.01886 & $0 / 18$ & 1 \\
Lead (mg/L) & $1 / 18$ & 0.5 & 0.5 & 0.2639 & 0.05893 & $0 / 18$ & 5 \\
$\begin{array}{l}\text { Mercury (mg/L) } \\
\text { Tetrachlorothene }\end{array}$ & $1 / 18$ & 0.08 & 0.1 & 0.01889 & 0.0261 & $0 / 18$ & 0.2 \\
$(\mu \mathrm{g} / \mathrm{L})$ & 88 & 88 & 21.28 & 22.25 & $0 / 18$ & 700 \\
\hline
\end{tabular}

${ }^{a}$ Nondetects were set to $1 / 2$ the detection limit and included in the average and standard deviation. 
Table B-1. Construction data for groundwater monitoring wells located at Kerr Hollow Quarry

\begin{tabular}{ccccccccc}
\hline Well & North & East & Elevation $^{a}$ & TOC $^{b}$ & $\begin{array}{c}\text { Screened or } \\
\text { open interval }^{c}\end{array}$ & TD $^{c}$ & $\begin{array}{c}\text { Screen } \\
\text { present }\end{array}$ & Aquifer zone \\
\hline GW 142 & 24524 & 64030 & 968.29 & 970.35 & $248.5-295$ & 295 & No & BDK \\
GW 143 & 24257 & 63522 & 911.04 & 913.18 & $205-253$ & 253 & No & BDK \\
GW 144 & 24255 & 63502 & 910.48 & 913.34 & $150-190$ & 195 & Yes & BDK \\
GW 145 & 24441 & 63266 & 837.92 & 840.04 & $88.5-108.5$ & 110 & Yes & BDK \\
GW 146 & 24432 & 63272 & 837.00 & 838.16 & $190-220$ & 220 & No & BDK \\
GW 147 & 24731 & 63428 & 848.41 & 851.62 & $53-68.1$ & 69 & Yes & UNC/BDK \\
GW 231 & 24725 & 63410 & 846.90 & 849.47 & $24.5-34.5$ & 35 & Yes & UNC/BDK \\
\hline
\end{tabular}

${ }^{a}$ Elevation reported is ground surface adjacent to well in feet above mean sea level

${ }^{b}$ Top of casing measured in feet above mean sea level.

c All downhole measurements in feet below ground surface.

BDK $=$ Bedrock

UNC $=$ Unconsolidated

Source: Jones et al. 1994 
Table B-2. Kerr Hollow Quarry drinking water parameter results for CY 1986

\begin{tabular}{|c|c|c|c|c|c|c|c|}
\hline Qtr & GW-142 & GW-143 & GW-144 & GW-145 & GW-146 & GW-147 & GW-231 \\
\hline \multicolumn{8}{|c|}{ 2,4,5-TP (SIVEX) (UG/L) } \\
\hline 1986Q1 & $<0.1$ & $<0.2$ & $<0.1$ & $<0.1$ & $<0.1$ & $<0.1$ & $<0.1$ \\
\hline 1986Q2 & $<0.2$ & $<0.2$ & $<0.2$ & $<0.2$ & $<0.2$ & $<0.2$ & $<0.2$ \\
\hline 1986Q3 & $<0.2$ & $<0.2$ & $<0.2$ & $<0.2$ & $<0.2$ & $<0.2$ & $<0.2$ \\
\hline 1986Q4 & $<0.1$ & $<0.1$ & $<0.1$ & $<0.1$ & $<0.1$ & $<0.1$ & $<0.1$ \\
\hline \multicolumn{8}{|c|}{$2,4-D(U G / L)$} \\
\hline 1986Q1 & $<1$ & $<2$ & $<1$ & $<1$ & $<1$ & $<1$ & $<1$ \\
\hline 1986Q2 & $<2$ & $<2$ & $<2$ & $<2$ & $<2$ & $<2$ & $<2$ \\
\hline 1986Q3 & $<2$ & $<2$ & $<2$ & $<2$ & $<2$ & $<2$ & $<2$ \\
\hline 1986Q4 & $<1$ & $<1$ & $<1$ & $<1$ & $<1$ & $<1$ & $<1$ \\
\hline \multicolumn{8}{|c|}{ ARSENIC $(M G / L)$} \\
\hline 1986Q1 & $<0.005$ & $<0.005$ & $<0.005$ & $<0.005$ & $<0.005$ & $<0.005$ & $<0.005$ \\
\hline 1986Q2 & $<0.005$ & $<0.005$ & $<0.005$ & 0.005 & $<0.005$ & $<0.005$ & $<0.005$ \\
\hline 1986Q3 & $<0.005$ & $<0.005$ & $<0.005$ & $<0.005$ & $<0.005$ & $<0.005$ & $<0.005$ \\
\hline 1986Q4 & $<0.005$ & $<0.005$ & $<0.005$ & $<0.005$ & $<0.005$ & $<0.005$ & $<0.005$ \\
\hline \multicolumn{8}{|c|}{ BARIUM (MG/L) } \\
\hline 1986Q1 & 0.14 & 0.033 & 0.034 & 0.077 & 0.0024 & 0.087 & 0.08 \\
\hline 1986Q2 & 0.13 & 0.025 & 0.035 & 0.065 & 0.0081 & 0.059 & 0.094 \\
\hline 1986Q3 & 0.1 & 0.014 & 0.039 & 0.073 & 0.019 & 0.072 & 0.12 \\
\hline 1986Q4 & 0.19 & 0.05 & 0.039 & 0.098 & 0.035 & 0.09 & 0.15 \\
\hline
\end{tabular}


Table B-2 (continued)

\begin{tabular}{|c|c|c|c|c|c|c|c|}
\hline Qtr & GW-142 & GW-143 & GW-144 & GW-145 & GW-146 & GW-147 & GW-231 \\
\hline \multicolumn{8}{|c|}{ CADMIUM $(M G / L)$} \\
\hline 1986Q1 & $<0.003$ & $<0.003$ & $<0.003$ & $<0.003$ & $<0.003$ & $<0.003$ & $<0.003$ \\
\hline 1986Q2 & $<0.003$ & $<0.003$ & $<0.003$ & $<0.003$ & $<0.003$ & $<0.003$ & $<0.003$ \\
\hline 1986Q3 & $<0.003$ & $<0.003$ & $<0.003$ & $<0.003$ & $<0.003$ & $<0.003$ & $<0.003$ \\
\hline 1986Q4 & $<0.003$ & $<0.003$ & $<0.003$ & $<0.003$ & $<0.003$ & $<0.003$ & $<0.003$ \\
\hline \multicolumn{8}{|c|}{ CHROMIUM (MG/L) } \\
\hline 1986Q1 & $<0.01$ & $<0.01$ & $<0.01$ & $<0.01$ & $<0.01$ & $<0.01$ & $<0.01$ \\
\hline 1986Q2 & $<0.01$ & $<0.01$ & $<0.01$ & $<0.01$ & $<0.01$ & $<0.01$ & $<0.01$ \\
\hline 1986Q3 & $<0.01$ & $<0.01$ & $<0.01$ & $<0.01$ & $<0.01$ & $<0.01$ & $<0.01$ \\
\hline 1986Q4 & 0.011 & $<0.01$ & $<0.01$ & 0.011 & $<0.01$ & $<0.01$ & $<0.01$ \\
\hline \multicolumn{8}{|c|}{ ENDRIN (UG/L) } \\
\hline 1986Q1 & $<0.05$ & $<0.05$ & $<0.05$ & $<0.05$ & $<0.05$ & $<0.05$ & $<0.05$ \\
\hline 1986Q2 & $<0.1$ & $<0.1$ & $<0.05$ & $<0.1$ & $<0.1$ & $<0.05$ & $<0.1$ \\
\hline 1986Q3 & $<0.1$ & $<0.1$ & $<0.1$ & $<0.1$ & $<0.1$ & $<0.1$ & $<0.1$ \\
\hline 1986Q4 & $<0.05$ & $<0.05$ & $<0.05$ & $<0.05$ & $<0.05$ & $<0.05$ & $<0.05$ \\
\hline \multicolumn{8}{|c|}{ FLUORIDE (MG/L) } \\
\hline 1986Q1 & 0.39 & 3.18 & 0.15 & 1.87 & 1.5 & 0.27 & 0.094 \\
\hline 1986Q2 & 0.5 & 3.5 & 0.1 & 1.9 & 2 & 0.5 & $<0.1$ \\
\hline 1986Q3 & 0.1 & 2.7 & 0.1 & 1.4 & 2 & $<0.1$ & $<0.11$ \\
\hline 1986Q4 & 0.3 & 2.8 & 0.1 & 1.9 & 1.8 & 0.1 & $<0.1$ \\
\hline
\end{tabular}


Table B-2 (continued)

\begin{tabular}{|c|c|c|c|c|c|c|c|}
\hline Qtr & GW-142 & GW-143 & GW-144 & GW-145 & GW-146 & GW-147 & GW-231 \\
\hline \multicolumn{8}{|c|}{ GROSS ALPHA (PCI/L) } \\
\hline 1986Q1 & 32 & 1 & 2 & 2 & 3.9 & 5 & $<0.1$ \\
\hline 1986Q2 & $<1$ & $<1$ & $<1$ & 2.46 & 2.16 & 3.27 & $<1$ \\
\hline 1986Q3 & $<1$ & $<2$ & 5 & 8 & 18 & $<2$ & $<2$ \\
\hline 1986Q4 & 13 & 17 & 10 & 14 & 4 & 15 & 12 \\
\hline \multicolumn{8}{|c|}{ GROSS BETA $(P C I / L)$} \\
\hline 1986Q1 & 51 & 12 & 3 & 150 & 92.4 & 5 & 3.5 \\
\hline 1986Q2 & 4.01 & 16.22 & $<2$ & 61.13 & 41.71 & 13.87 & 4.14 \\
\hline 1986Q3 & 6 & 12 & 9 & 65 & 44 & 8 & 2 \\
\hline 1986Q4 & 16 & 33 & 12 & 39 & 43 & 18 & 19 \\
\hline \multicolumn{8}{|c|}{$L E A D(M G / L)$} \\
\hline 1986Q1 & 0.027 & $<0.004$ & $<0.004$ & $<0.004$ & $<0.004$ & 0.006 & $<0.004$ \\
\hline 1986Q2 & 0.016 & 0.019 & 0.011 & 0.017 & 0.008 & 0.008 & 0.011 \\
\hline 1986Q3 & 0.004 & 0.004 & 0.007 & 0.004 & 0.023 & $<0.004$ & 0.004 \\
\hline 1986Q4 & 0.005 & $<0.004$ & 0.017 & 0.009 & $<0.004$ & $<0.005$ & $<0.004$ \\
\hline \multicolumn{8}{|c|}{ MERCURY $(M G / L)$} \\
\hline 1986Q1 & $<0.0002$ & $<0.0002$ & $<0.0002$ & $<0.0002$ & $<0.0002$ & $<0.0002$ & $<0.0002$ \\
\hline 1986Q2 & $<0.0002$ & $<0.0002$ & $<0.0002$ & $<0.0002$ & $<0.0002$ & $<0.0002$ & $<0.0002$ \\
\hline 1986Q3 & $<0.0002$ & $<0.0002$ & $<0.0002$ & $<0.0002$ & $<0.0002$ & $<0.0002$ & $<0.0002$ \\
\hline $1986 Q 4$ & $<0.0002$ & $<0.0002$ & $<0.0002$ & $<0.0002$ & $<0.0002$ & $<0.0002$ & $<0.0002$ \\
\hline
\end{tabular}


Table B-2 (continued)

\begin{tabular}{|c|c|c|c|c|c|c|c|}
\hline Qtr & GW-142 & GW-143 & GW-144 & GW-145 & GW-146 & GW-147 & GW-231 \\
\hline \multicolumn{8}{|c|}{ METHOXYCHLOR (UG/L) } \\
\hline 1986Q1 & $<0.04$ & $<0.04$ & $<0.04$ & $<0.04$ & $<0.04$ & $<0.04$ & $<0.04$ \\
\hline 1986Q2 & $<0.08$ & $<0.08$ & $<0.04$ & $<0.08$ & $<0.08$ & $<0.04$ & $<0.08$ \\
\hline 1986Q3 & $<0.08$ & $<0.08$ & $<0.08$ & $<0.08$ & $<0.08$ & $<0.08$ & $<0.08$ \\
\hline 1986Q4 & $<0.04$ & $<0.04$ & $<0.04$ & $<0.04$ & $<0.04$ & $<0.04$ & $<0.04$ \\
\hline \multicolumn{8}{|c|}{ NITRATE AS N (MG/L) } \\
\hline 1986Q1 & 0.27 & $<0.11$ & 0.59 & 0.11 & 0.14 & 0.5 & 0.21 \\
\hline 1986Q2 & 0.23 & $<0.11$ & 0.47 & $<0.11$ & 0.11 & 0.43 & 0.16 \\
\hline 1986Q3 & $<0.11$ & $<0.11$ & 0.28 & 0.25 & $<0.11$ & 0.75 & $<0.11$ \\
\hline 1986Q4 & 0.33 & $<0.11$ & 0.41 & 0.29 & $<0.11$ & 0.63 & $<0.11$ \\
\hline \multicolumn{8}{|c|}{$R A D I U M(B Q / L)$} \\
\hline 1986Q1 & $<0.1$ & $<0.1$ & $<0.1$ & $<0.1$ & $<0.1$ & $<0.1$ & $<0.1$ \\
\hline 1986Q2 & $<0.1$ & $<0.1$ & $<0.1$ & $<0.1$ & $<0.1$ & $<0.1$ & $<0.1$ \\
\hline 1986Q3 & 0.28 & $<0.1$ & $<0.1$ & $<0.1$ & 0.54 & $<0.1$ & 0.51 \\
\hline 1986Q4 & $<0.1$ & $<0.1$ & $<0.1$ & $<0.1$ & $<0.1$ & $<0.1$ & $<0.1$ \\
\hline \multicolumn{8}{|c|}{ SELENIUM (MG/L) } \\
\hline 1986Q1 & $<0.005$ & $<0.005$ & $<0.005$ & 0.009 & $<0.005$ & $<0.005$ & $<0.005$ \\
\hline 1986Q2 & $<0.005$ & $<0.005$ & $<0.005$ & $<0.005$ & 0.006 & $<0.005$ & $<0.005$ \\
\hline 1986Q3 & $<0.005$ & $<0.005$ & $<0.005$ & $<0.005$ & $<0.005$ & $<0.005$ & $<0.005$ \\
\hline 1986Q4 & $<0.005$ & $<0.005$ & $<0.005$ & $<0.005$ & $<0.005$ & $<0.005$ & $<0.005$ \\
\hline
\end{tabular}


Table B-2 (continued)

\begin{tabular}{|c|c|c|c|c|c|c|c|}
\hline Qtr & GW-142 & GW-143 & GW-144 & GW-145 & GW-146 & GW-147 & GW-231 \\
\hline \multicolumn{8}{|c|}{$S L V E R(M G / L)$} \\
\hline 1986Q1 & $<0.006$ & $<0.006$ & $<0.006$ & $<0.006$ & $<0.006$ & $<0.006$ & $<0.006$ \\
\hline 1986Q2 & $<0.006$ & $<0.006$ & $<0.006$ & $<0.006$ & $<0.006$ & $<0.006$ & $<0.006$ \\
\hline 1986Q3 & $<0.006$ & $<0.006$ & $<0.006$ & $<0.006$ & $<0.006$ & $<0.006$ & $<0.006$ \\
\hline 1986Q4 & $<0.006$ & $<0.006$ & $<0.006$ & $<0.006$ & $<0.006$ & $<0.006$ & $<0.006$ \\
\hline \multicolumn{8}{|c|}{ TOXAPHENE (UG/L) } \\
\hline 1986Q1 & $<1$ & $<1$ & $<1$ & $<1$ & $<1$ & $<1$ & $<1$ \\
\hline 1986Q2 & $<2$ & $<2$ & $<1$ & $<2$ & $<2$ & $<1$ & $<2$ \\
\hline 1986Q3 & $<2$ & $<2$ & $<2$ & $<2$ & $<2$ & $<2$ & $<2$ \\
\hline 1986Q4 & $<1$ & $<1$ & $<1$ & $<1$ & $<1$ & $<1$ & $<1$ \\
\hline
\end{tabular}


Table B-3. Kerr Hollow Quarry interim status detection monitoring parameters

First year (1986)

Primary drinking water standards

Arsenic

Barium

Cadmium

Chromium

Fluoride

Lead

Mercury

Nitrate

Selenium

Silver

Endrin

Lindane

Methoxychlor

Toxaphene

2-4-D

2,4,5-TP

Radium

Gross alpha

Gross beta

Indicator parameters

$\mathrm{pH}$

Specific conductance

Total organic carbon

Total organic halides

Parameters establishing groundwater quality

Chloride

Iron

Manganese

Phenols

Sodium

Sulfate 
Table B-3 (continued)

Analyze for

Second and subsequent years (1987 through 1994)

Indicator parameters ${ }^{1}$

$\mathrm{pH}$

Specific Conductance

Total Organic Carbon

Total Organic Halides

Parameters establishing groundwater quality ${ }^{2}$

Chloride

Iron

Manganese

Phenols

Sodium

Sulfate

Other analytes analyzed (1987)

Trace metals ${ }^{3}$

ICP analysis

Potassium

Fluorimetric analysis

Uranium

Volatile organic compounds

Acetone

Benzene

Bromodichloromethane

Bromoform

Bromomethane

2-Butanone

Carbon disulfide

Carbon tetrachloride

Chlorobenzene

Chlorodibromomethane

Chloroethane

2-Chloroethylvinyl ether

Chloroform

Chloromethane

1,1-Dichloroethane

1,2-Dichloroethane 
Table B-3 (continued)

\begin{tabular}{|c|c|}
\hline Parameter & Analyze for \\
\hline & 1,1-Dichloroethene \\
\hline & trans-1,2-Dichloroethene \\
\hline & 1,2-Dichloropropane \\
\hline & cis-1,3-Dichloropropene \\
\hline & trans-1,3-Dichloropropene \\
\hline & Ethylbenzene \\
\hline & 2-Hexanone \\
\hline & 4-Methyl-2-pentanone \\
\hline & Methylene chloride \\
\hline & Styrene \\
\hline & 1,1,2,2-Tetrachloroethane \\
\hline & Tetrachloroethene \\
\hline & Toluene \\
\hline & 1,1,1-Trichloroethane \\
\hline & 1,1,2-Trichloroethane \\
\hline & Trichloroethene \\
\hline & Vinyl acetate \\
\hline & Vinyl chloride \\
\hline & Xylenes \\
\hline \multirow[t]{13}{*}{ Acid and base neutral extractable organics } & Acenaphthylene \\
\hline & bis (2-Chloroethyl) ether \\
\hline & 2-Chlorophenol \\
\hline & 1,3-Dichlorobenzene \\
\hline & 1,4-Dichlorobenzene \\
\hline & Benzyl alcohol \\
\hline & 1,2-Dichlorobenzene \\
\hline & 2-Methylphenol \\
\hline & bis (2-Chloroisopropyl) ether \\
\hline & 4-Methylphenol \\
\hline & N-Nitrosodi-N-propylamine \\
\hline & Hexachloroethane \\
\hline & Nitrobenzene \\
\hline
\end{tabular}


Table B-3 (continued)

\begin{tabular}{|c|c|}
\hline Parameter & Analyze for \\
\hline & Isophorone \\
\hline & 2-Nitrophenol \\
\hline & 2,4-Dimethylphenol \\
\hline & Benzoic acid \\
\hline & bis (2-Chloroethoxy) methane \\
\hline & 2,4-Dichlorophenol \\
\hline & 1,2,4-Trichlorobenzene \\
\hline & Naphthalene \\
\hline & 4-Chloroaniline \\
\hline & Hexachlorobutadiene \\
\hline & 4-Chloro-3-methylphenol \\
\hline & 2-Methylnaphthalene \\
\hline & Hexachlorocyclopentadiene \\
\hline & 2,4,6-Trichlorophenol \\
\hline & 2,4,5-Trichlorophenol \\
\hline & 2-Chloronaphthalene \\
\hline & 2-Nitroaniline \\
\hline & Dimethylphthalate \\
\hline \multirow[t]{3}{*}{ Major ions } & Alkalinity - carbonate \\
\hline & Alkalinity - Bicarbonate \\
\hline & Nitrate (as N) \\
\hline \multirow[t]{4}{*}{ Field measurements } & Temperature \\
\hline & Dissolved oxygen \\
\hline & Static water level \\
\hline & $\begin{array}{l}\text { Oxidation ređuction potential } \\
\text { (REDOX) }\end{array}$ \\
\hline \multicolumn{2}{|l|}{ Other analytes analyzed (1988) } \\
\hline \multirow[t]{5}{*}{ Trace metals ${ }^{3}$} & ICP analysis \\
\hline & Aluminum \\
\hline & Antimony \\
\hline & Arsenic \\
\hline & Barium \\
\hline
\end{tabular}


Table B-3 (continued)

\begin{tabular}{|c|c|}
\hline Parameter & Analyze for \\
\hline & Beryllium \\
\hline & Boron \\
\hline & Calcium \\
\hline & Cadmium \\
\hline & Chromium \\
\hline & Cobalt \\
\hline & Copper \\
\hline & Magnesium \\
\hline & Molybdenum \\
\hline & Nickel \\
\hline & Niobium \\
\hline & Phosphorous \\
\hline & Potassium \\
\hline & Selenium \\
\hline & Silicon \\
\hline & Silver \\
\hline & Strontium \\
\hline & Thorium \\
\hline & Titanium \\
\hline & Vanadium \\
\hline & Zinc \\
\hline & Zirconium \\
\hline & Fluorimetric analysis \\
\hline & Uranium \\
\hline \multirow[t]{3}{*}{ Major ions } & Alkalinity - carbonate \\
\hline & Alkalinity - Bicarbonate \\
\hline & Nitrate (as N) \\
\hline \multirow[t]{2}{*}{ Miscellaneous parameters } & Total suspended solids (TSS) \\
\hline & Total dissolved solids (TDS) \\
\hline \multirow[t]{3}{*}{ Field measurements } & Temperature \\
\hline & Dissolved oxygen \\
\hline & Static water level \\
\hline
\end{tabular}


Table B-3 (continued)

\begin{tabular}{cc}
\hline Parameter & Analyze for \\
\hline & Oxidation reduction potential \\
(REDOX)
\end{tabular}

Other analytes analyzed (1989)

Trace metals ${ }^{3}$

ICP analysis

Aluminum

Antimony

Arsenic

Barium

Beryllium

Boron

Calcium

Cadmium

Chromium

Cobalt

Copper

Lead

Magnesium

Molybdenum

Nickel

Potassium

Selenium

Silicon

Silver

Strontium

Thorium

Vanadium

Zinc

AAS analysis

Cadmium

Fluorimetric analysis

Uranium 
Table B-3 (continued)

\begin{tabular}{|c|c|}
\hline Parameter & Analyze for \\
\hline \multirow[t]{3}{*}{ Major ions } & Alkalinity - carbonate \\
\hline & Alkalinity - Bicarbonate \\
\hline & Nitrate (as N) \\
\hline \multirow[t]{3}{*}{ Miscellaneous parameters } & Total suspended solids (TSS) \\
\hline & Total dissolved solids (TDS) \\
\hline & Turbidity \\
\hline \multirow[t]{4}{*}{ Field measurements } & Temperature \\
\hline & Dissolved oxygen \\
\hline & Static water level \\
\hline & $\begin{array}{l}\text { Oxidation reduction potential } \\
\text { (REDOX) }\end{array}$ \\
\hline \multicolumn{2}{|c|}{ Other analytes analyzed (1990 through 1994) } \\
\hline \multirow[t]{20}{*}{ Trace metals ${ }^{3}$} & ICP analysis \\
\hline & Aluminum \\
\hline & Antimony \\
\hline & Arsenic \\
\hline & Barium \\
\hline & Beryllium \\
\hline & Boron \\
\hline & Calcium \\
\hline & Cadmium \\
\hline & Chromium \\
\hline & Cobalt \\
\hline & Copper \\
\hline & Magnesium \\
\hline & Molybdenum \\
\hline & Nickel \\
\hline & Potassium \\
\hline & Selenium \\
\hline & Silicon ${ }^{4}$ \\
\hline & $A A S$ analysis \\
\hline & Cadmium \\
\hline
\end{tabular}


Table B-3 (continued)

\begin{tabular}{|c|c|}
\hline Parameter & Analyze for \\
\hline & Chromium \\
\hline & Lead \\
\hline & Cold vapor atomic absorption \\
\hline & Mercury \\
\hline & Fluorimetric analysis \\
\hline & Uranium \\
\hline \multirow[t]{26}{*}{ Volatile organic compounds } & Acetone \\
\hline & Benzene \\
\hline & Bromodichloromethane \\
\hline & Bromoform \\
\hline & Bromoethane \\
\hline & 2-Butanone \\
\hline & Carbon disulfide \\
\hline & Carbon tetrachloride \\
\hline & Chlorobenzene \\
\hline & Chlorodibromomethane \\
\hline & Chloroethane \\
\hline & Chloroform \\
\hline & Chloromethane \\
\hline & 1,1-Dichloroethane \\
\hline & 1,2-Dichloroethane \\
\hline & 1,1-Dichoroethene \\
\hline & 1,2-Dichlorethene \\
\hline & 1,2-Dichloropropane \\
\hline & cis-1,3-Dichloropropene \\
\hline & trans-1,3-Dichloropropene \\
\hline & Ethylbenzene \\
\hline & 2-Hexanone \\
\hline & 4-Methyl-2-pentanone \\
\hline & Methylene chloride \\
\hline & Styrene \\
\hline & 1,1,2,2-Tetrachloroethane \\
\hline & $\cdot$ \\
\hline
\end{tabular}


Table B-3 (continued)

\begin{tabular}{|c|c|}
\hline Parameter & Analyze for \\
\hline & Tetrachloroethene \\
\hline & Toluene \\
\hline & 1,1,1-Trichloroethane \\
\hline & 1,1,2-Trichloroethane \\
\hline & Trichloroethene \\
\hline & Vinyl acetone \\
\hline & Vinyl chloride \\
\hline & Xylenes \\
\hline \multirow[t]{4}{*}{ Major ions } & Alkalinity - carbonate \\
\hline & Alkalinity - Bicarbonate \\
\hline & Fluoride \\
\hline & Nitrate (as N) \\
\hline \multirow[t]{4}{*}{ Miscellaneous parameters } & $\mathrm{pH}$ \\
\hline & Total suspended solids (TSS) \\
\hline & Total dissolved solids (TDS) \\
\hline & Turbidity \\
\hline \multirow[t]{2}{*}{ Radiological parameters } & Gross alpha activity \\
\hline & Gross beta activity \\
\hline \multirow[t]{4}{*}{ Field measurements } & Temperature \\
\hline & Dissolved oxygen \\
\hline & Static water level \\
\hline & $\begin{array}{l}\text { Oxidation reduction potential } \\
\text { (REDOX) }\end{array}$ \\
\hline
\end{tabular}

1 Analyzed semi-annually

2 Analyzed annually

3 ICP - Inductively coupled plasma spectroscopy AAS - Atomic absorption spectroscopy

4 Silicon was analyzed for in 1990 and 1991 only 
Table B-4. Summary of statistically significant results at the Kerr Hollow Quarry for 1987 through 1994

\begin{tabular}{ll}
\hline Test & Results \\
\hline
\end{tabular}

1987 Results

Total organic carbon

pH (pH units)

GW-142

GW-144

GW-144

GW-231

GW-231

Conductivity ( $\mu \mathrm{mhos} / \mathrm{cm})$

GW-145

\section{Results}

Total organic carbon

$\mathrm{pH}$ (pH units)

GW-142

GW-142

GW-144

GW-144

GW-231

GW-231

\section{Results}

Total organic carbon

$\mathrm{pH}$ (pH units)

GW-142
Unable to perform t-test

Significantly less than 1986 upgradient (7.6/8.2), Q4

Significantly less than 1986 upgradient (7.6/8.2), Q4

Significantly less than 1986 (7.6/7.9), Q4

Significantly less than 1986 upgradient (7.6/8.2), Q2 Significantly less than 1986 upgradient (7.5/8.2), Q4

Significantly less than 1986 (7.5/7.8), Q4

Significantly greater than 1986 (783/602), Q2

Unable to perform t-test

Significantly less than 1986 (7.1/8.2), Q2

Significantly less than 1986 upgradient (7.1/8.3), Q2

Significantly less than 1986 (7.4/8.0), Q2 Significantly less than 1986 (7.6/8.0), Q4

Significantly less than 1986 upgradient (7.4/8.3), Q2 Significantly less than 1986 upgradient (7.6/8.3), Q4

Significantly less than 1986 (7.5/7.9), Q2

Significantly less than 1986 (7.5/7.9), Q4

Significantly less than 1986 upgradient (7.5/8.3), Q2 Significantly less than 1986 upgradient (7.5/8.3), Q4

Unable to perform t-test

Significantly less than 1986 (6.9/8.2), Q1 Significantly less than 1986 (6.7/8.2), Q3 
Table B-4 (continued)

\begin{tabular}{cl}
\hline Test & \multicolumn{1}{c}{ Results } \\
\hline GW-142 & Significantly less than 1986 upgradient (6.9/8.3), Q1 \\
& Significantly less than 1986 upgradient (6.7/8.3), Q3 \\
GW-144 & Significantly less than $1986(6.6 / 8.0), Q 1$ \\
& Significantly less than $1986(6.5 / 8.0), Q 3$ \\
GW-144 & Significantly less than 1986 upgradient (6.6/8.3), Q1 \\
& Significantly less than 1986 upgradient $(6.5 / 8.3), Q 3$ \\
GW-145 & Significantly less than $1986(7.2 / 8.2), Q 1$ \\
& Significantly less than $1986(6.7 / 8.2), Q 3$ \\
GW-231 & Significantly less than $1986(6.5 / 7.9), Q 1$ \\
& Significantly less than $1986(6.5 / 7.9), Q 3$ \\
GW-231 & Significantly less than 1986 upgradient $(6.5 / 8.3), Q 1$ \\
& Significantly less than 1986 upgradient (6.5/8.3), Q3
\end{tabular}

1990 Results

$\mathrm{pH}$ (pH units)

GW-142

Significantly less than 1986 (7.0/8.2), Q1

Significantly less than 1986 (7.2/8.2), Q4

GW-142

Significantly less than 1986 upgradient (7.0/8.3), Q1 Significantly less than 1986 upgradient (7.2/8.3), Q4

GW-144

Significantly less than 1986 (6.9/8.0), Q1 Significantly less than 1986 (6.9/8.0), Q4

GW-144

Significantly less than 1986 upgradient (6.9/8.3), Q1 Significantly less than 1986 upgradient (6.9/8.3), Q4

GW-145

Significantly less than 1986 (7.2/8.3), Q1 Significantly less than 1986 (7.3/8.3), Q4

GW-231

Significantly less than 1986 (7.0/7.9), Q1

Significantly less than 1986 (6.9/7.9), Q4

GW-231

Significantly less than 1986 upgradient (7.0/8.3), Q1 Significantly less than 1986 upgradient (6.9/8.3), Q4

1991 Results

Total organic carbon

GW-142

Significantly greater than 1987 upgradient (3.75/1.43)

$\mathrm{pH}$ (pH units)

GW-142

Significantly less than 1986 (7.1/8.2), Q1

GW-142

Significantly less than 1986 upgradient (7.1/8.3), Q1

95-044P/051995 
Table B-4 (continued)

\begin{tabular}{ll}
\hline Test & \multicolumn{1}{c}{ Results } \\
\hline GW-144 & $\begin{array}{l}\text { Significantly less than } 1986(6.9 / 8.0), Q 1 \\
\text { Significantly less than } 1986(7.8 / 8.0), Q 3\end{array}$ \\
GW-144 & Significantly less than 1986 upgradient $(6.9 / 8.3), Q 1$ \\
GW-145 & Significantly less than $1986(7.1 / 8.3), Q 1$ \\
GW-231 & Significantly less than $1986(6.9 / 7.9), Q 1$ \\
& Significantly less than $1986(7.4 / 7.9), Q 3$ \\
GW-231 & Significantly less than 1986 upgradient $(6.9 / 8.3), Q 1$ \\
& Significantly less than 1986 upgradient $(7.4 / 8.3), Q 3$
\end{tabular}

Conductivity ( $\mu \mathrm{mhos} / \mathrm{cm})$

GW-142

Significantly greater than 1986 (440/351), Q1

Significantly greater than 1986 (462/351), Q3

1992 Results

Total organic carbon

GW-145

Significantly greater than $1987(9.10 / 1.43)$, Q3

$\mathrm{pH}$ (pH units)

GW-142

Significantly less than 1986 upgradient (7.7/8.3), Q3

GW-144

Significantly less than 1986 (7.8/8.0), Q1

GW-231

Significantly less than 1986 upgradient (7.7/8.3), Q3

Conductivity ( $\mu \mathrm{mhos} / \mathrm{cm})$

GW-142

Significantly greater than 1986 (447/351), Q1

1993 Results

Total organic carbon

GW-142

Significantly greater than 1987 upgradient (3.25/1.43), Q2

GW-145

Significantly greater than 1987 (6.75/1.43), Q2

$\mathrm{pH}$ (pH units)

GW-144

Significantly greater than $1986(8.3 / 8.0)$, Q2

GW-231

Significantly less than 1986 (7.5/7.9), Q1

GW-231

Significantly less than 1986 upgradient (7.5/8.3), Q1

Significantly less than 1986 upgradient (7.7/8.3), Q4 


\begin{tabular}{|c|c|}
\hline Test & Results \\
\hline \multicolumn{2}{|c|}{ Conductivity ( $\mu$ mhos $/ \mathrm{cm})$} \\
\hline GW-142 & $\begin{array}{l}\text { Significantly greater than } 1986(441 / 351), \text { Q1 } \\
\text { Significantly greater than } 1986(446 / 351), \text { Q2 } \\
\text { Significantly greater than } 1986(442 / 351), \text { Q3 }\end{array}$ \\
\hline \multicolumn{2}{|l|}{1994 Results } \\
\hline \multicolumn{2}{|c|}{ Total organic carbon $(\mathrm{mg} / \mathrm{L})$} \\
\hline GW-145 & $\begin{array}{l}\text { Significantly greater than } 1987(5.50 / 1.43), \text { Q2 } \\
\text { Significantly greater than } 1987(3.68 / 1.43), \text { Q3 } \\
\text { Significantly greater than } 1987(4.00 / 1.43), \text { Q4 }\end{array}$ \\
\hline \multicolumn{2}{|l|}{$\mathrm{pH}$ (pH units) } \\
\hline GW-142 & Significantly less than 1986 upgradient (7.7/8.3), Q4 \\
\hline GW-144 & $\begin{array}{l}\text { Significantly greater than } 1986(8.2 / 8.0), \text { Q1 } \\
\text { Significantly less than } 1986(7.8 / 8.0) \text {, Q2 } \\
\text { Significantly less than } 1986(7.8 / 8.0), \text { Q4 }\end{array}$ \\
\hline GW-231 & $\begin{array}{l}\text { Significantly less than } 1986(7.4 / 7.9), \text { Q2 } \\
\text { Significantly less than } 1986(7.5 / 7.9), Q 3 \\
\text { Significantly less than } 1986(7.8 / 7.9), \text { Q4 }\end{array}$ \\
\hline GW-231 & $\begin{array}{l}\text { Significantly less than } 1986 \text { upgradient }(7.7 / 8.3), \text { Q1 } \\
\text { Significantly less than } 1986 \text { upgradient }(7.4 / 8.3) \text {, Q2 } \\
\text { Significantly less than } 1986 \text { upgradient }(7.5 / 8.3), \text { Q3 } \\
\text { Significantly less than } 1986 \text { upgradient }(7.5 / 8.3), Q 4\end{array}$ \\
\hline \multicolumn{2}{|c|}{ Conductivity ( $\mu \mathrm{mhos} / \mathrm{cm})$} \\
\hline GW-142 & $\begin{array}{l}\text { Significantly greater than } 1986(439 / 351), \text { Q1 } \\
\text { Significantly greater than } 1986(449 / 351), \text { Q2 }\end{array}$ \\
\hline
\end{tabular}

Note: Values in parenthesis present the current value compared to the reference value. For example, "significantly less than 1986 upgradient (7.6/8.2), Q4" shows the fourth quarter result of 7.6 compared to the 1986 upgradient reference value of 8.2 .

$\begin{array}{ll}\text { Q1 } & \text { 1st Quarter } \\ \text { Q2 } & \text { 2nd Quarter } \\ \text { Q3 } & \text { 3rd Quarter } \\ \text { Q4 } & \text { 4th Quarter }\end{array}$ 
Table B-5. Kerr Hollow Quarry 1988 Appendix IX constituent detections

\begin{tabular}{lcccccc}
\hline & GW-142 & GW-143 & GW-144 & GW-145 & GW-147 & GW-231 \\
\hline $\begin{array}{l}\text { Total Elemental } \\
\text { Analysis-Metals (mg/l) }\end{array}$ & & & & & & \\
Barium & 0.45 & $\mathrm{U}$ & $\mathrm{U}$ & 0.216 & 0.216 & 0.253 \\
Chromium & $\mathrm{U}$ & $\mathrm{U}$ & $\mathrm{U}$ & 0.011 & $\mathrm{U}$ & $\mathrm{U}$ \\
Lead & 0.0056 & $\mathrm{U}$ & 0.008 & 0.0408 & 0.0293 & $\mathrm{U}$
\end{tabular}

HSL VOCs $(\mu \mathrm{g} / \mathrm{l})$

Methylene Chloride

Acetone

Chloroform

Carbon Tetrachloride

Benzene

Toluene

Fluorotrichloromethane

$30 \quad 31$

2

2

2

33

6

U

6

U U

1

U

$\mathrm{U}$

$\mathrm{U} . \mathrm{U}$

5

U

U

U

U

2 U

U

U

U

U

23

2

$\mathrm{U}$

U

U

U

$5 \quad 5$

5

U

U

13

U

U

$\mathrm{U}$

Semi-volatile HSL $(\mu \mathrm{g} / \mathrm{l})$.

Di-n-Butylphthalate

U U

$\mathrm{U}$

6

2

2

1

3

bis-2-Ethylhexylphthalate

2

8

13

12

74

\section{Herbicides/Pesticides \\ $(\mu \mathrm{g} / \mathrm{l})$}

beta-BHC

U

U

0.16

U

0.07

0.03

Source: Weston 1989

HSL $=$ Hazardous substance list

$\mathbf{U}=$ analyte was analyzed for but not detected 
Table B-6. Summary of field and laboratory residuum permeability test results

\begin{tabular}{ccccccc}
\hline $\begin{array}{c}\text { Range of } \\
\text { depth tested } \\
(\mathrm{ft})\end{array}$ & $\begin{array}{c}\text { Typical } \\
\text { depth(s) tested } \\
(\mathrm{ft})\end{array}$ & $\begin{array}{c}\text { Mean field } \\
\text { hydraulic conductivity } \\
(\mathrm{ft} / \mathrm{d})\end{array}$ & $\begin{array}{c}\text { Mean laboratory } \\
\text { Number of tests }\end{array}$ & $\begin{array}{c}\text { hydraulic conductivity } \\
\text { (ft/d) }\end{array}$ & Number of tests & Reference \\
\hline $0-25$ & 10 & $1.7 \times 10^{-2}$ & 21 & $9.1 \times 10^{-3}$ & 12 & $a$ \\
& $5-25$ & $2.7 \times 10^{-1}$ & 6 & $6.4 \times 10^{-4}$ & 3 & $b$ \\
& $0-6$ & 1.8 & 16 & No data & 0 & 3 \\
$25-60$ & 40 & $5.7 \times 10^{-3}$ & 18 & $2.8 \times 10^{-4}$ & 3 & $a$ \\
& $25-60$ & $4.3 \times 10^{-1}$ & 8 & $2.8 \times 10^{-5}$ & 7 & $b$ \\
$60-100$ & $70-100$ & No data & 0 & $1.8 \times 10^{-4}$ & 8 & $a$ \\
& $60-85$ & $4.9 \times 10^{-1}$ & 4 & No data & 0 & $b$ \\
\hline
\end{tabular}

${ }^{a}$ Woodward Clyde 1984

${ }^{b}$ Mishu 1982

${ }^{c}$ Results are for vertical permeameter test.

Modified from Ketelle and Huff 1984, Mishu 1982. 
Table B-7. Summary of packer test results for core holes GW-131, GW-135, and CH-157 ${ }^{a}$

\begin{tabular}{|c|c|c|}
\hline Test number & Tested depth interval (ft) & Hydraulic conductivity (ft/d) \\
\hline CH-157-10 & $145-157$ & $2.4 \times 10^{-1}$ \\
\hline $\mathrm{CH}-157-1$ & $215-227$ & $5.01 \times 10^{-1}$ \\
\hline $\mathrm{CH}-157-2$ & $265-277$ & 3.03 \\
\hline $\mathrm{CH}-157-3$ & $282-294$ & $5.61 \times 10^{-1}$ \\
\hline $\mathrm{CH}-157-4$ & $314-326$ & $9.13 \times 10^{-3}$ \\
\hline $\mathrm{CH}-157-5$ & $326-338$ & $8.90 \times 10^{-1}$ \\
\hline $\mathrm{CH}-157-6$ & $344-356$ & $2.18 \times 10^{-4}$ \\
\hline $\mathrm{CH}-157-7$ & $392-404$ & $8.28 \times 10^{-4}$ \\
\hline $\mathrm{CH}-157-8$ & $432-444$ & $2.09 \times 10^{-1}$ \\
\hline $\mathrm{CH}-157-9$ & $468-480$ & $4.17 \times 10^{-1}$ \\
\hline GW-135-1 & $190-217$ & $1.56 \times 10^{-2}$ \\
\hline GW-135-2 & $324-351$ & $2.03 \times 10^{-1}$ \\
\hline GW-135-3 & $397-424$ & $2.72 \times 10^{-1}$ \\
\hline GW-135-4 & $446-473$ & $2.22 \times 10^{-1}$ \\
\hline GW-135-5 & $588-615$ & $1.01 \times 10^{-2}$ \\
\hline GW-131-10 & $120-147$ & 1.31 \\
\hline GW-131-1 & $240-267$ & 3.20 \\
\hline GW-131-2 & $290-317$ & $2.08 \times 10^{-4}$ \\
\hline GW-131-3 & $370-397$ & $1.24 \times 10^{-1}$ \\
\hline GW-131-4 & $450-477$ & $5.44 \times 10^{-1}$ \\
\hline GW-131-5 & $490-517$ & $3.23 \times 10^{-3}$ \\
\hline
\end{tabular}

${ }^{a} \mathrm{GW}-131$ and GW-135 are designated as GW-series wells, as opposed to the CH-classification for core hole $\mathrm{CH}-157$, because they were subsequently equipped with multi-port monitoring devices.

Modified from King and Haase 1988. 
Table B-8. Summary of average cation and anion concentrations detected in groundwater samples collected during CY 1994 from Kerr Hollow Quarry monitoring wells

\begin{tabular}{lccccc}
\hline Well no. & $\begin{array}{c}\text { Calcium } \\
\text { (meq/L) }\end{array}$ & $\begin{array}{c}\text { Magnesium } \\
(\text { meq/L) }\end{array}$ & $\begin{array}{c}\text { Potassium } \\
\text { (meq/L) }\end{array}$ & $\begin{array}{c}\text { Sodium } \\
\text { (meq/L) }\end{array}$ & \\
\hline GW-142 & 2.20 & 2.57 & 0.10 & 0.043 & \\
GW-143 & 1.54 & 2.02 & 0.45 & 1.05 & \\
GW-144 & 1.93 & 1.15 & 0.05 & 0.05 & \\
GW-145 & 2.10 & 2.82 & 0.32 & 0.33 & \\
GW-146 & 2.04 & 2.80 & 0.43 & 0.55 & \\
GW-147 & 1.55 & 1.48 & 0.18 & 0.14 & \\
GW-231 & 1.58 & 1.31 & 0.04 & 0.05 & \\
\cline { 2 - 6 } & HCO & Chloride & Fluoride & Nitrate-N & Sulfate \\
$($ meq/L) & (meq/L) & (meq/L) \\
\hline (meq/L) & 0.05 & 0.03 & 0.00 & 0.12 \\
GW-142 & 4.70 & 0.19 & 0.16 & 0.01 & 0.80 \\
GW-143 & 4.06 & 0.06 & 0.01 & 0.12 & 0.14 \\
GW-144 & 3.04 & 0.26 & 0.14 & 0.01 & 0.71 \\
GW-145 & 4.23 & 0.35 & 0.15 & 0.02 & 1.38 \\
GW-146 & 4.03 & 0.06 & 0.02 & 0.09 & 0.08 \\
GW-147 & 3.14 & 0.06 & 0.00 & 0.06 & 0.07 \\
GW-231 & 2.84 & & & & \\
\hline
\end{tabular}

$\mathrm{meq} / \mathrm{L}=$ milliequivalent per liter

Source: HSW, Inc. 1995 
Table B-9. Public users of groundwater within 10 miles of the Kerr Hollow Quarry ${ }^{a}$

\begin{tabular}{lrrll}
\hline \multicolumn{1}{c}{ Public water user } & $\begin{array}{r}\text { People } \\
\text { served }\end{array}$ & Yield (gpd) & Source & $\begin{array}{l}\text { Probable water- } \\
\text { bearing formation }\end{array}$ \\
\hline Oliver Springs & 4,000 & $2.5 \times 10^{5}$ & Spring & Knox \\
Dutch Valley Elementary School & 140 & $2.7 \times 10^{3}$ & Well & Rome \\
First Utility District of & 3,600 & $2.7 \times 10^{5}$ & Spring & Conasauga \\
Anderson County & & & & \\
West Knox Utility District & 15,000 & $1.3 \times 10^{6}$ & Well & Knox \\
Dixie-Lee Utility District & 6,700 & $4.1 \times 10^{5}$ & Spring & Knox \\
Edgewood SE Center & 100 & $4.3 \times 10^{3}$ & Well & Knox \\
Cumberland Utility District of & 4,300 & $1.7 \times 10^{5}$ & Spring & Knox \\
Roane and Morgan Counties & & & & \\
Charles H. Bacon Co. & & $8.4 \times 10^{4}$ & Well & Knox \\
\hline
\end{tabular}

${ }^{a}$ Union Carbide Corporation, Nuclear Division (1982) and personal communication between H. King and D. Rosowitz (1994).

${ }^{b}$ Half supply.

$c$ Primary source. 
Table B-10. Validated VOC results from Kerr Hollow Quarry well sampling since first quarter CY 1990 (in $\mu \mathrm{g} / \mathrm{L}$ )

\begin{tabular}{|c|c|c|c|c|c|}
\hline \multirow{2}{*}{$\frac{\text { Well }}{\text { Quarter/Year }}$} & \multicolumn{2}{|c|}{ GW-142 } & \multicolumn{3}{|c|}{ GW-144 } \\
\hline & Chloroform & Tetrachloroethene & $\begin{array}{c}\text { Carbon } \\
\text { Tetrachloride }\end{array}$ & Chloroform & Tetrachloroethene \\
\hline Q4/94 & $\mathrm{U}$ & $\mathrm{U}$ & U & $\mathrm{U}$ & $\mathrm{U}$ \\
\hline Q3/94 & $\mathbf{U}$ & $\mathbf{U}$ & $\mathbf{U}$ & $\mathbf{U}$ & U \\
\hline Q2/94 & $\mathrm{U}$ & $U$ & 2.0 & $\mathrm{U}$ & $\mathbf{U}$ \\
\hline Q1/94 & $\mathrm{U}$ & $\mathrm{U}$ & $\mathbf{U}$ & $\mathrm{U}$ & $\mathrm{U}$ \\
\hline Q4/93 & 4.0 & $\mathrm{U}$ & 3.0 & $\mathrm{U}$ & $\mathbf{U}$ \\
\hline $\mathrm{Q} 3 / 93$ & $\mathrm{U}$ & $\mathrm{U}$ & 3.0 & $\mathrm{U}$ & $\mathbf{U}$ \\
\hline $\mathrm{Q} 2 / 93$ & $\mathrm{U}$ & $\mathbf{U}$ & $\mathbf{U}$ & $\mathrm{U}$ & $\mathbf{U}$ \\
\hline Q1/93 & $\mathrm{U}$ & U & $\mathbf{U}$ & $\mathrm{U}$ & $\mathbf{U}$ \\
\hline $\mathrm{Q} 4 / 92$ & $\mathrm{U}$ & $U$ & 5.0 & 0.8 & $\mathbf{U}$ \\
\hline $\mathrm{Q} 3 / 92$ & $\mathrm{U}$ & $\mathbf{U}$ & 2.0 & $U$ & $\mathbf{U}$ \\
\hline $\mathrm{Q} 2 / 92$ & $\mathrm{U}$ & 2.0 & 2.0 & $\mathbf{U}$ & $\mathbf{U}$ \\
\hline Q1/92 & $\mathrm{U}$ & $U$ & U & $\mathrm{U}$ & $\mathrm{U}$ \\
\hline Q4/91 & $\mathrm{U}$ & 0.8 & 1.0 & $\mathrm{U}$ & 1.0 \\
\hline $\mathrm{Q} 3 / 91$ & $\mathrm{U}$ & 0.7 & 2.0 & 2.0 & 2.0 \\
\hline Q2/91 & $\mathrm{U}$ & $\mathbf{U}$ & 5.0 & 1.0 & $U$ \\
\hline Q1/91 & $\mathrm{U}$ & 0.7 & $\mathbf{U}$ & U & $\mathbf{U}$ \\
\hline Q4/90 & $\mathbf{U}$ & $\mathrm{U}$ & 2.0 & $U$ & $\mathbf{U}$ \\
\hline $\mathrm{Q} 3 / 90$ & $\mathrm{U}$ & 0.9 & 6.0 & 1.0 & $\mathbf{U}$ \\
\hline $\mathrm{Q} 2 / 90$ & $\mathrm{U}$ & $\mathrm{U}$ & $\mathrm{U}$ & $\mathbf{U}$ & $\mathbf{U}$ \\
\hline Q1/90 & $\mathbf{U}$ & $\mathbf{U}$ & $\mathrm{U}$ & $\mathbf{U}$ & $\mathbf{U}$ \\
\hline
\end{tabular}

$\mathrm{U}=$ Not detected

Note: All results validated against laboratory and trip blanks 
Table B-11. Monitoring parameters and comparative values (values are $\mathrm{mg} / \mathrm{L}$ unless otherwise noted)

\begin{tabular}{lc}
\hline Parameter & Tolerance Limit \\
\hline Barium & $0.49^{1}$ \\
Boron & \multicolumn{2}{c}{${ }^{2}$} \\
Cadmium & $0.005^{3}$ \\
Chromium & $0.1^{3}$ \\
Lead & $0.035^{1}$ \\
Mercury & $0.002^{3}$ \\
Nickel & $0.1^{3}$ \\
Strontium (total) & -2 \\
Uranium (total) & $0.019^{l}$ \\
Gross Alpha & $15 \mathrm{pCi} / \mathrm{L}^{3}$ \\
Gross Beta & $4 \mathrm{mrem} / \mathrm{year}^{3}$ \\
Carbon Tetrachloride & $5 \mu \mathrm{g} / \mathrm{L}^{3}$ \\
Tetrachloroethene & $5 \mu \mathrm{g} / \mathrm{L}^{3}$ \\
Chloroform & $100 \mu \mathrm{g} / \mathrm{L}^{3,4}$ \\
\hline
\end{tabular}

$\mu \mathrm{g} / \mathrm{L} \quad$ micrograms per liter

$\mathrm{mg} / \mathrm{L}$ milligram per liter

$\mathrm{pCi} / \mathrm{L}$ picoCuries per liter

mrem millirem

MCL maximum contaminant level

${ }^{1}$ UTL

${ }^{2}$ Annual trending analysis

${ }^{3} \mathrm{MCL}$

${ }^{4} \mathrm{MCL}$ is for total trihalomethanes 


\begin{tabular}{|c|c|c|c|c|c|}
\hline Well Number: & GW-142 & GW-143 & GW-144 & GW-145 & GW-231 \\
\hline North Coordinates & 24524.00 & 24257.00 & 24255.00 & 24441.00 & 24725.00 \\
\hline East Coordinates & 64030.00 & 63522.00 & 63502.00 & 63266.00 & 63410.00 \\
\hline Surface Elevation (fl) & 968.29 & 911.04 & 910.48 & 837.29 & 846.90 \\
\hline Measuring Point Elevation (ft) & 970.35 & 913.18 & 913.34 & 840.04 & 849.47 \\
\hline Aquifer Zone & BDR & $\mathrm{BDR}$ & BDR & WT & BDR \\
\hline Geologic Formation & OCk & oCk & OCk & OCk & oCk \\
\hline Hydrostratigraphic Unit & $\mathrm{AQF}$ & $\mathrm{AQF}$ & $\mathrm{AQF}$ & $\mathrm{AQF}$ & $\mathrm{AQF}$ \\
\hline Top of Fresh Bedrock (ft bgs) & NA & 18.0 & 40.0 & 12.0 & 10.5 \\
\hline Total Drilled Depth (n bgs) & 295.0 & 253.0 & 195.0 & 110.0 & 35.0 \\
\hline Screen Or Open & open & open & screened & screened & screened \\
\hline $\begin{array}{l}\text { Top of Monitoring Interval (ft } \\
\text { bgs) }\end{array}$ & 248.5 & 205.0 & 148.0 & 86.0 & 22.8 \\
\hline $\begin{array}{l}\text { Bottom of Monitoring Interval } \\
\text { (ft bgs) }\end{array}$ & 295.0 & 253.0 & 195.0 & 110.0 & 35.0 \\
\hline Screen Material & NA & NA & $\mathrm{PVC} / \# 40$ & $\mathrm{PVC} / \# 40$ & $\mathrm{PVC} / \# 40$ \\
\hline Screen Slot Size (in) & NA & NA & $\mathrm{sw} / 0.01$ & $\mathrm{sw} / 0.01$ & $\mathrm{sw} / 0.01$ \\
\hline Screen Length (ft) & NA & NA & 40.0 & 20.0 & 10.0 \\
\hline Top Filter Pack (ft bgs) & NA & NA & 148.0 & 86.0 & 22.8 \\
\hline Bottom Filter Pack (fi bgs) & NA & NA & 195.0 & 110.0 & 35.0 \\
\hline Top Filter Pack (n, msl) & NA & NA & 765.34 & 754.04 & 826.67 \\
\hline Bottom Filter Pack (fl, msl) & NA & NA & 718.34 & 730.04 & 814.47 \\
\hline Cond. Borehole Depth (ft bgs) & 20.0 & 20.0 & 40.0 & NA & 35.0 \\
\hline Cond. Borehole Diameter (in) & 16.75 & 16.75 & 15.0 & NA & 11.00 \\
\hline
\end{tabular}




\begin{tabular}{|c|c|c|c|c|c|}
\hline Well Number: & GW-142 & GW-143 & GW-144 & GW-145 & GW-231 \\
\hline Conductor Casing Type & PVC/\#40 & $\mathrm{PVC} / \# 40$ & $\mathrm{PVC} / \mathrm{t} 40$ & $\mathrm{PVC} / \# 40$ & $\mathrm{PVC} / \# 40$ \\
\hline Conductor Casing O.D. (in) & 12.5 & 10.625 & 12.5 & 12.5 & 10.625 \\
\hline Conductor Casing I.D. (in) & 11.75 & 9.875 & 11.75 & 11.75 & 9.825 \\
\hline $\begin{array}{l}\text { Conductor Casing Depth (ft } \\
\text { bgs) }\end{array}$ & 20.0 & 20.0 & 40.0 & 12.0 & 11.0 \\
\hline Pilot Borehole Depth (ft bgs) & NA & NA & NA & NA & NA \\
\hline Pilot Borehole Diameter (in) & NA & NA & NA & NA & NA \\
\hline Inner Casing Type & Steel/F25 & Steel/F25 & $\mathrm{PVC} / \# 40$ & $\mathrm{PVC} / \pi 40$ & $\mathrm{PVC} / \# 40$ \\
\hline Inner Casing O.D. (in) & 6.625 & 6.625 & 4.5 & 4.5 & 4.5 \\
\hline Inner Casing I.D. (in) & 6.125 & 6.125 & 4.0 & 4.0 & 4.0 \\
\hline Inner Casing Depth (ft bgs) & 248.5 & 205.0 & 150.0 & 88.5 & 24.5 \\
\hline Date Completed & $030 c t 85$ & $240 c t 85$ & $240 c t 85$ & $140 c t 85$ & $020 c 185$ \\
\hline Reference & Y/TS-881/R2 & Y/TS-881/R2 & $\mathrm{Y} / \mathrm{TS}-881 / \mathrm{R} 2$ & Y/TS-881/R2 & Y/TS-881/R2 \\
\hline
\end{tabular}

\begin{tabular}{ll}
\hline \multicolumn{2}{l}{ Abbreviations } \\
BDR & Bedrock Interval \\
WT & Water Table Interval \\
ft & feet \\
AQF & Knox Aquifer \\
OCk & Knox Group \\
NA & Not Applicable or Not Available \\
bgs & below ground surface \\
in & inches \\
sw & spiral wound \\
PVC & polyvinyl chloride \\
msl & mean sea level
\end{tabular}

Y/TS-881/R2 Jones et al. 1994

Table B-12 (continued) 


\section{FIGURES}




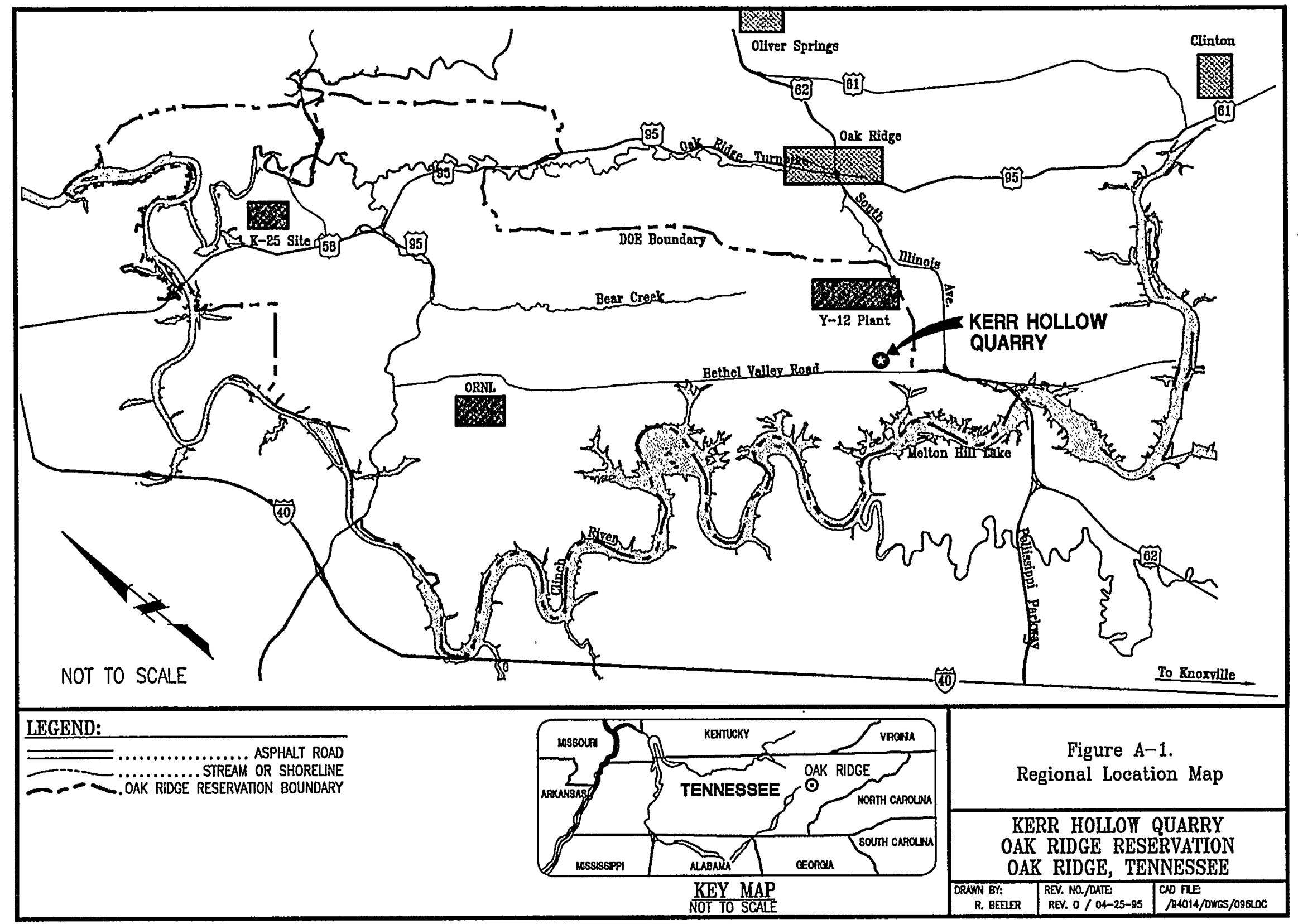




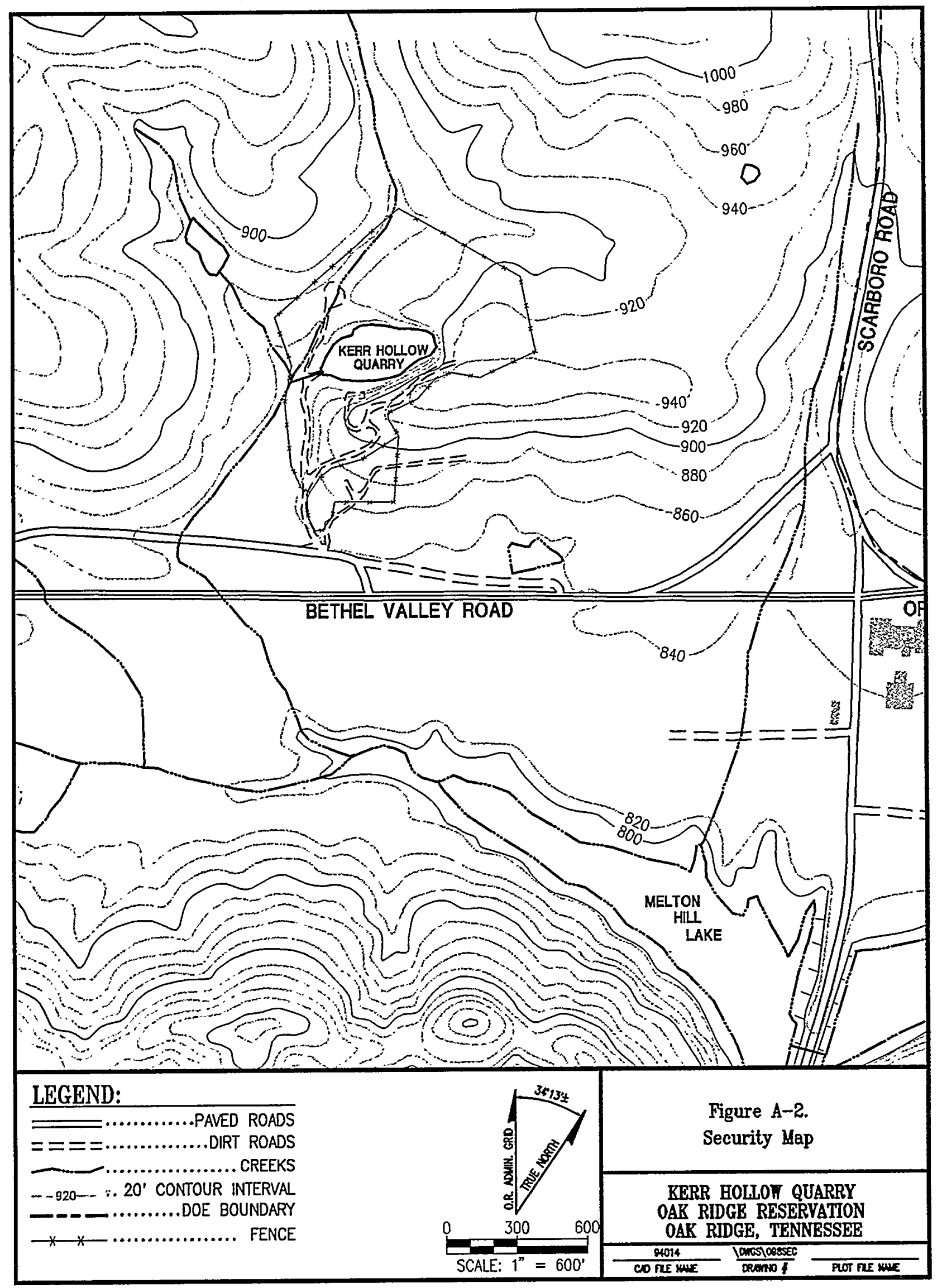




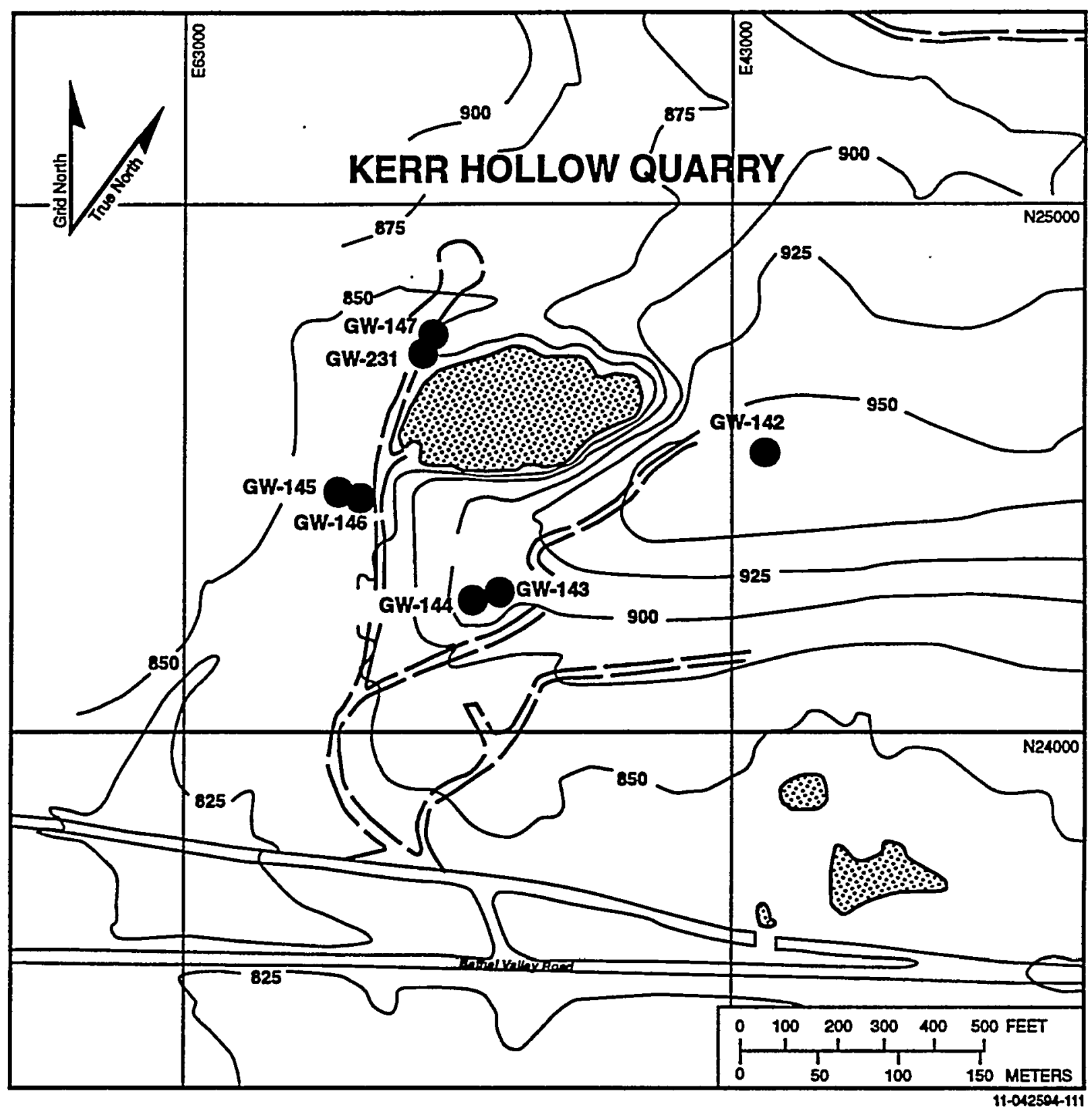

Figure B-1. Location of groundwater monitoring wells at Kerr Hollow Quarry. 


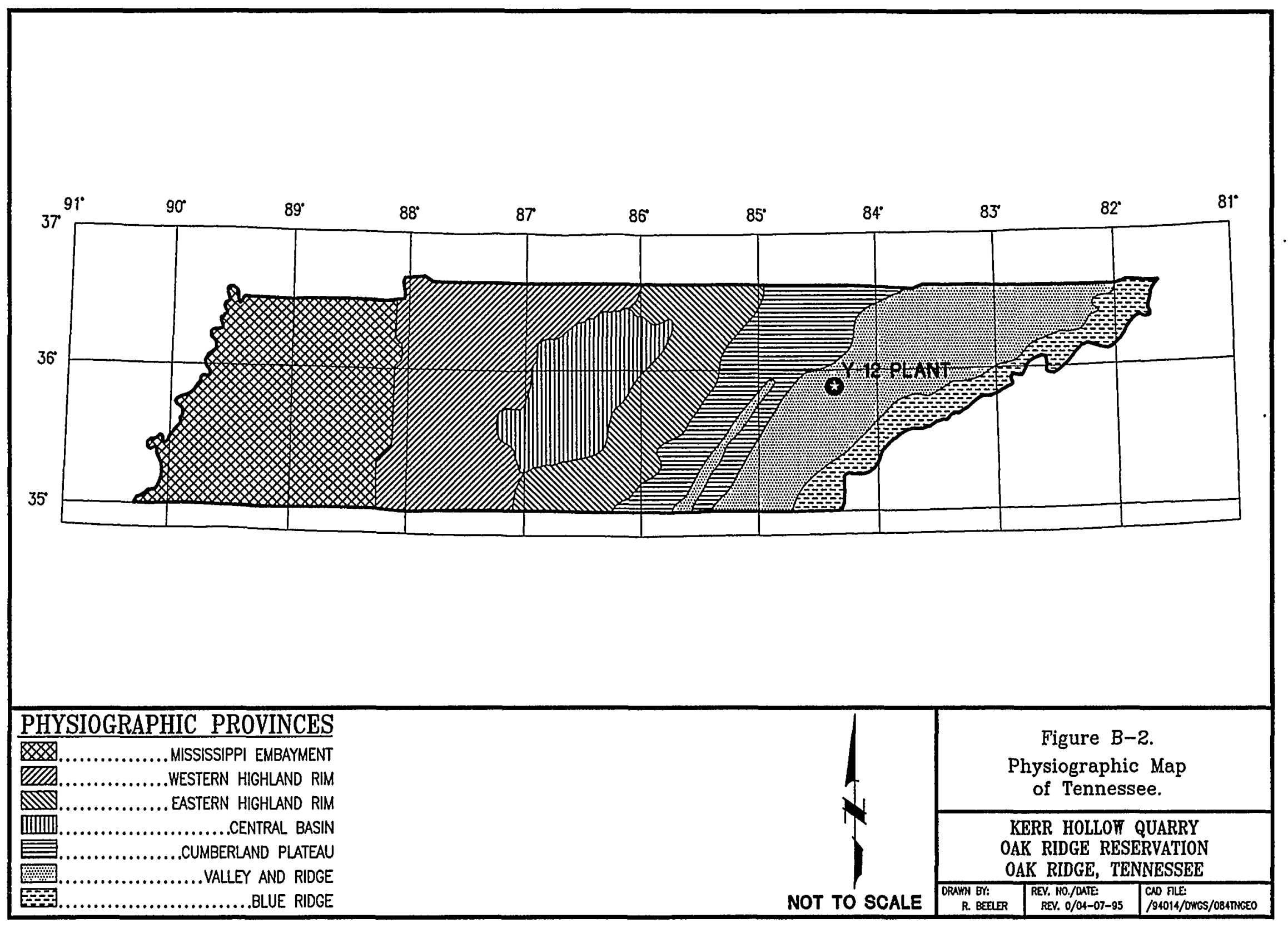




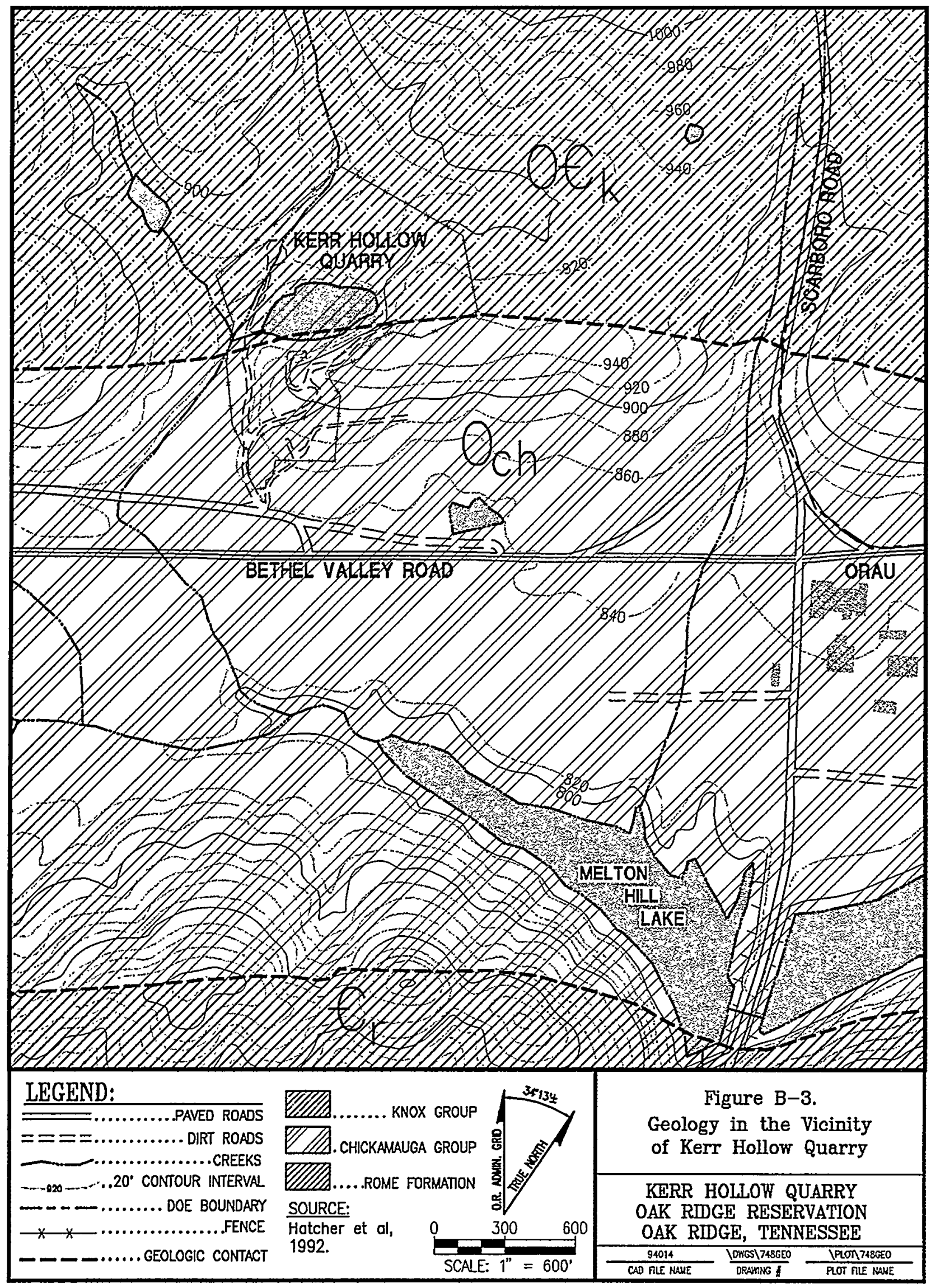




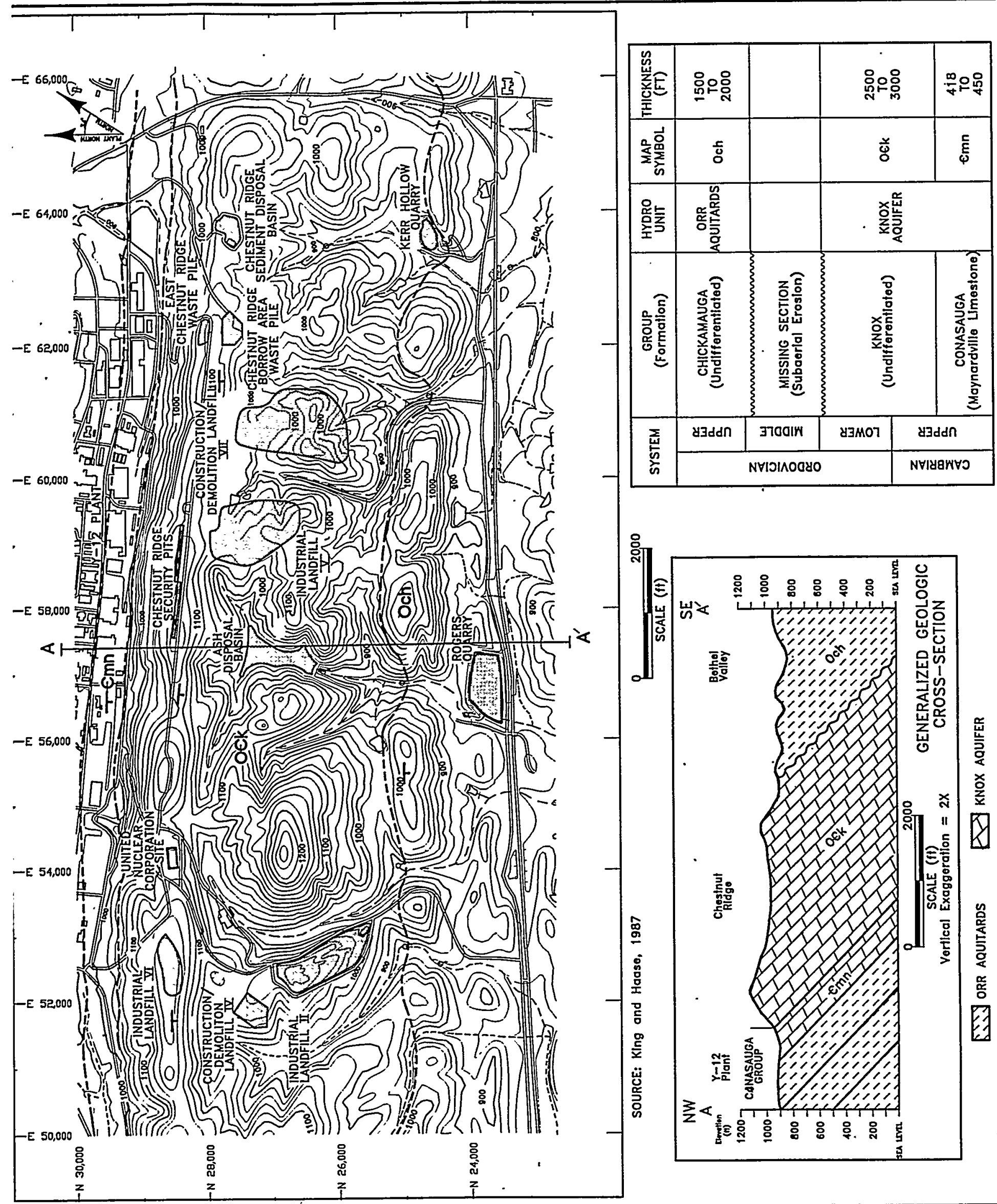

\begin{tabular}{r|c|}
\hline LOCATION: & $\begin{array}{c}Y-12 \text { PLANT } \\
\text { OAK RIDGE, TN. }\end{array}$ \\
\hline DATE: & $2-13-95$ \\
\hline DWG ID.: & OR211-HC \\
\hline
\end{tabular}

Figure B-4. Generalized geologic map of the Y-12 Plant area. 


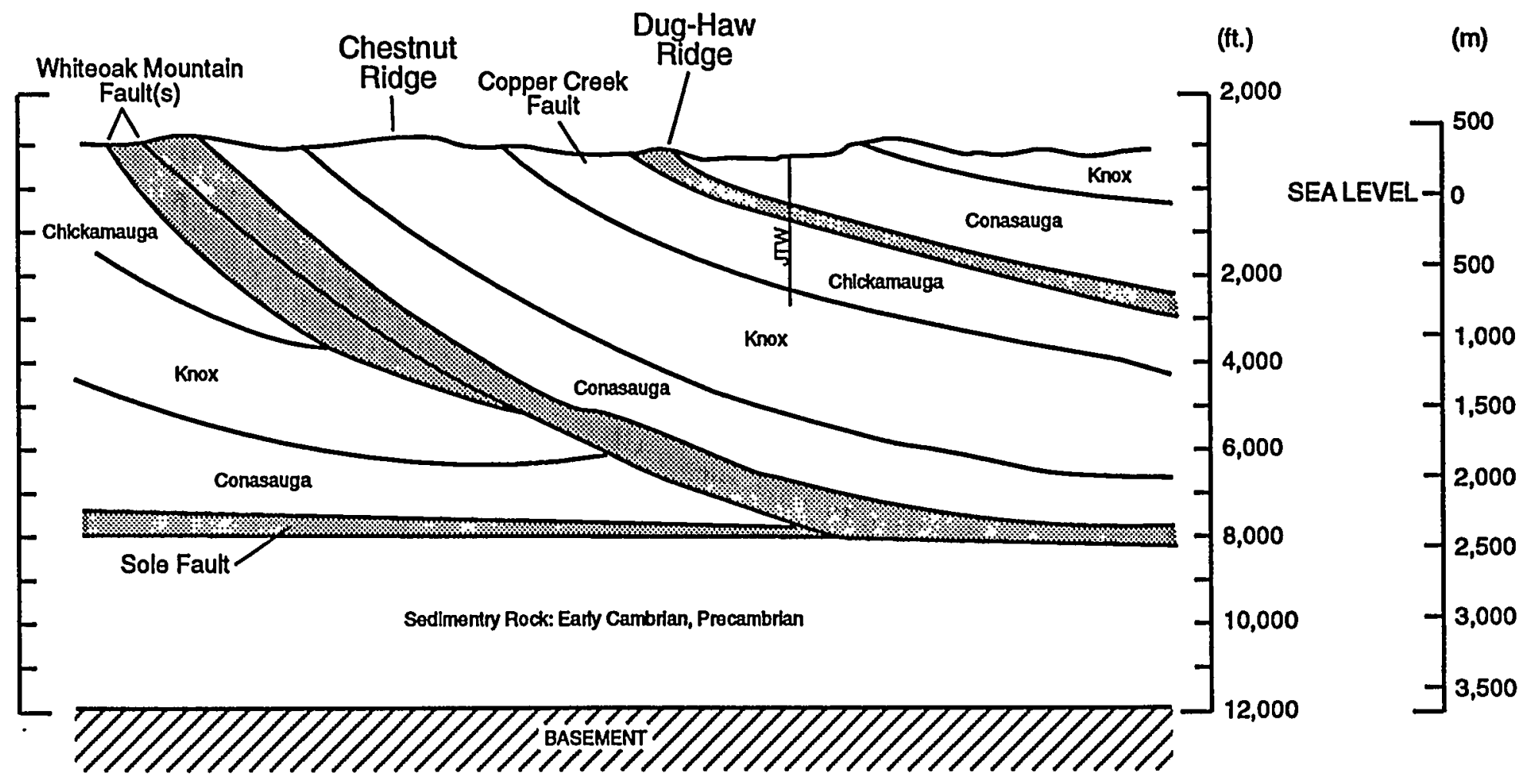

Figure B-5. Geologic cross section through the Chestnut Ridge Hydrogeologic Regime. 


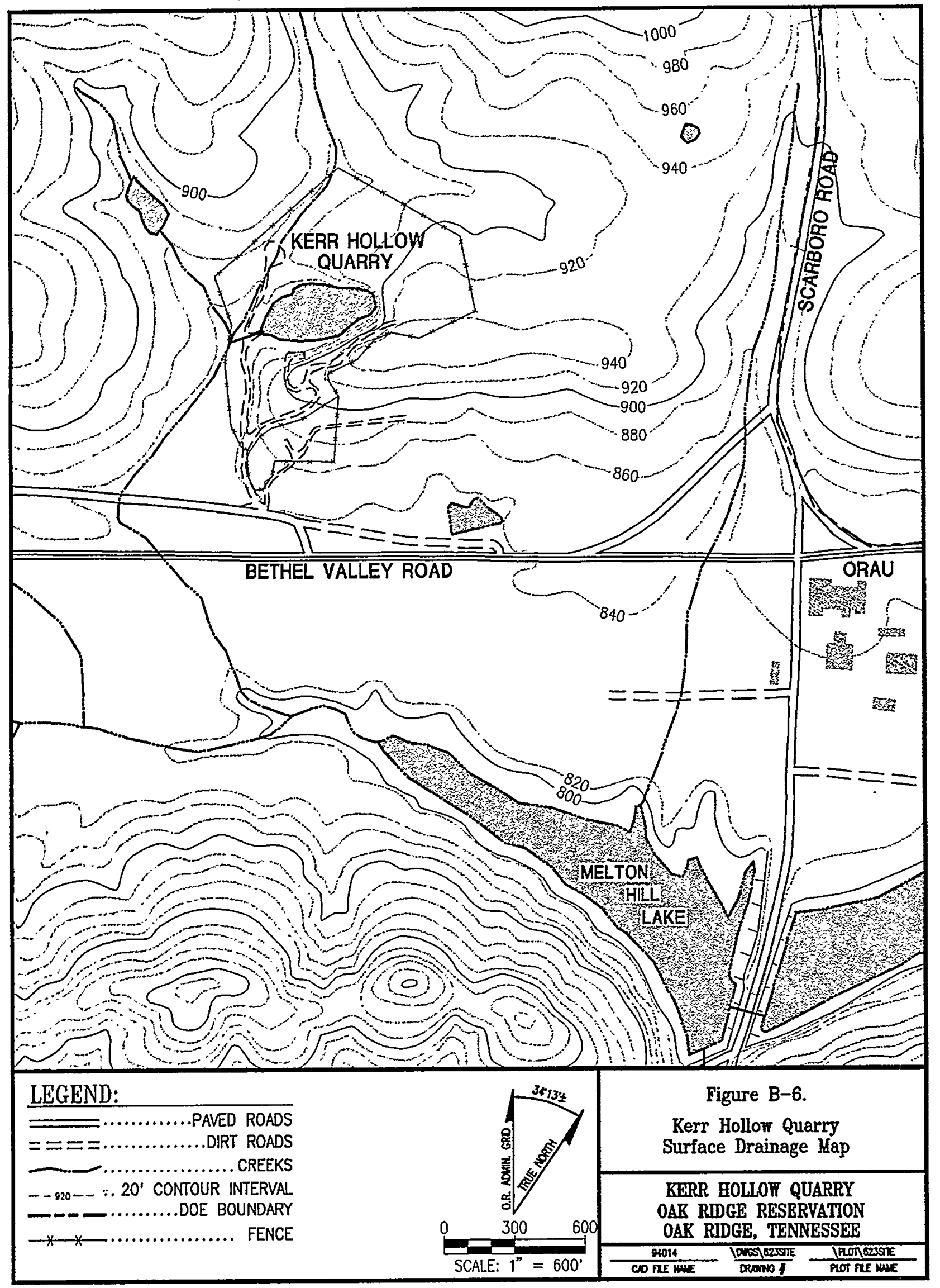




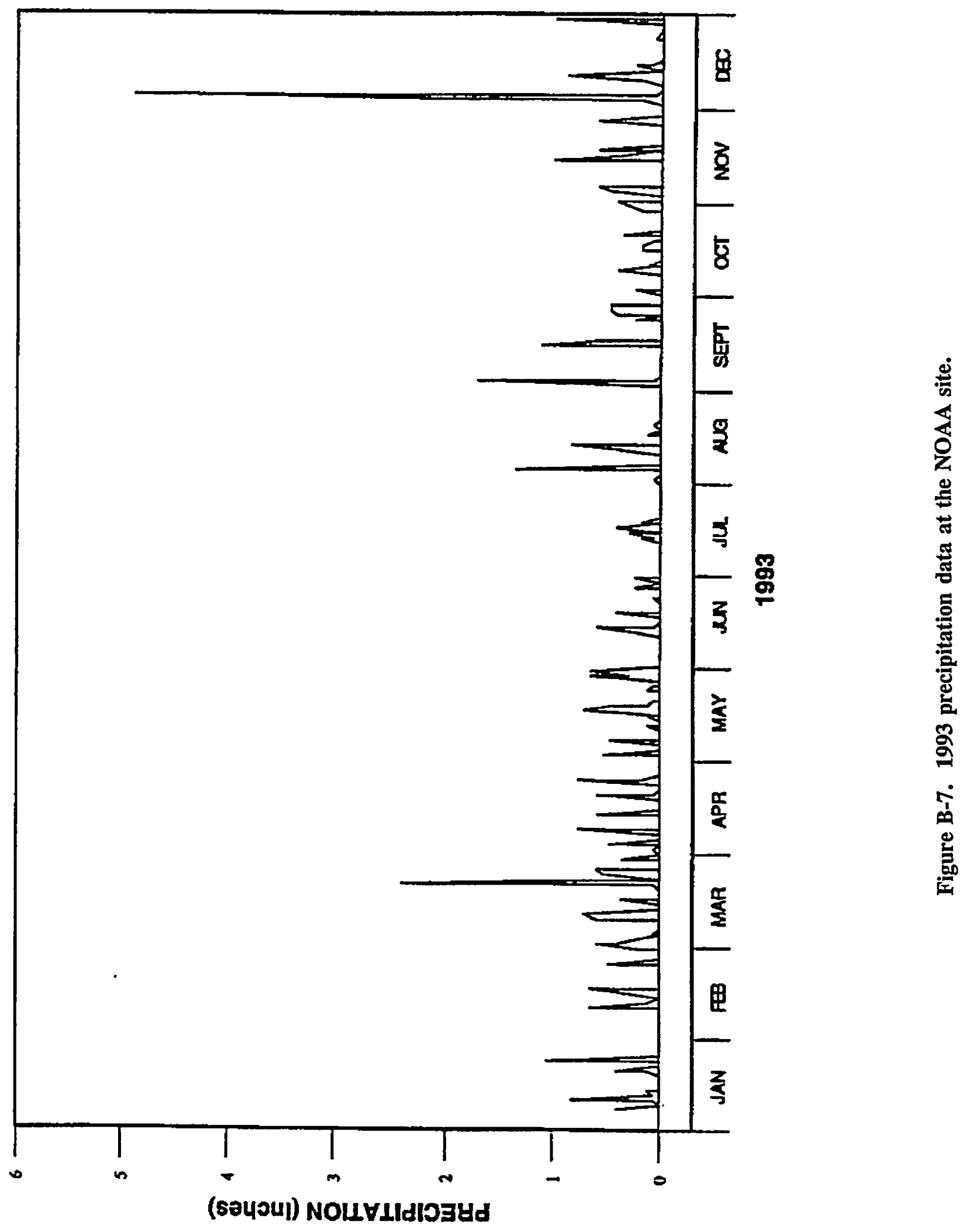


HYDROSTRATIGRAPHIC UNITS

PROPOSED BY SOLOMON et al. (1992)

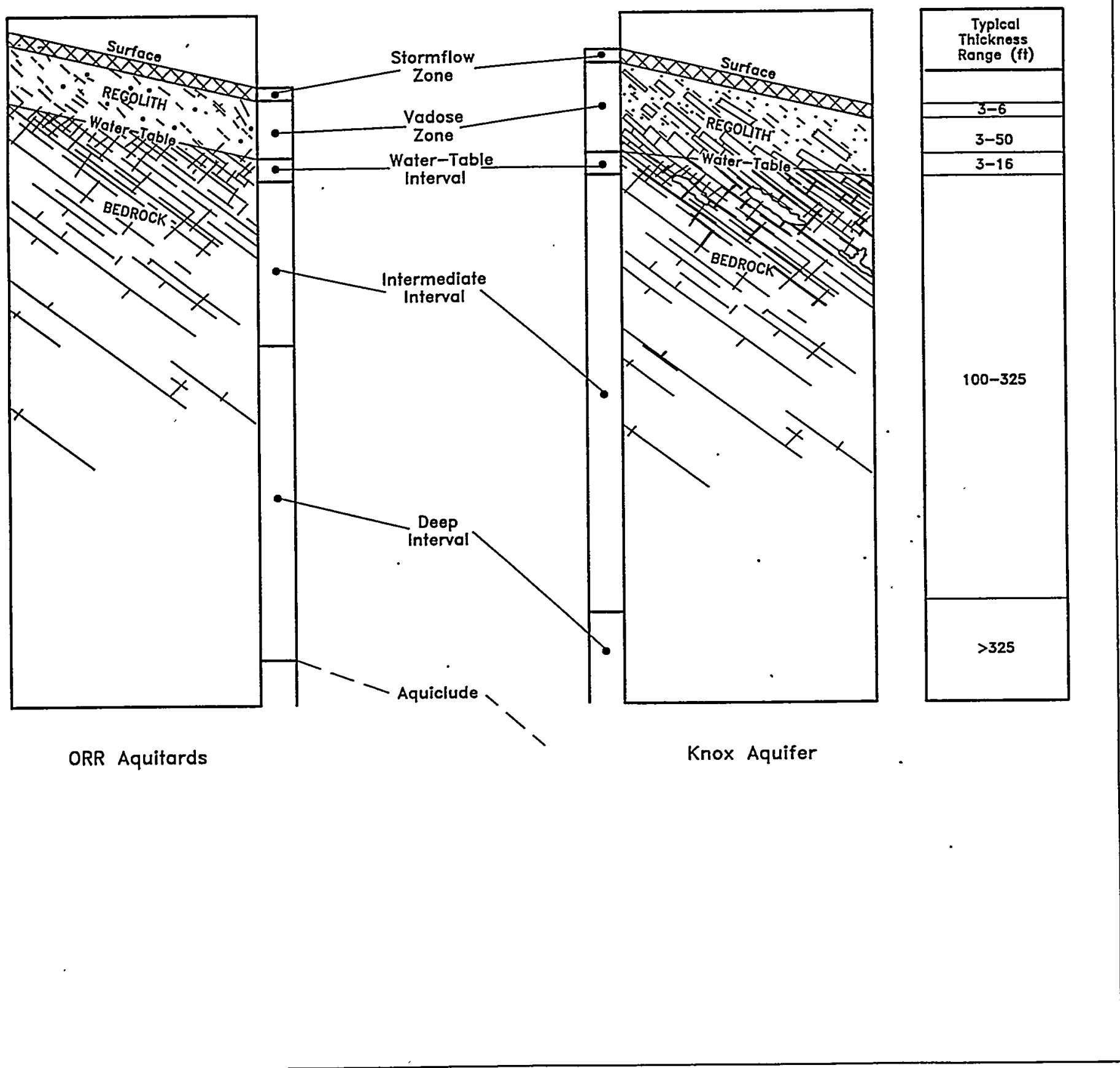

\begin{tabular}{r|c|}
\hline \multirow{2}{*}{ LOCATION: } & $\begin{array}{c}\text { Y-12 PLANT } \\
\text { OAK RIDGE, TN. }\end{array}$ \\
\hline DATE: & $1-18-95$ \\
\hline DWG ID.: & OR213-HC \\
\hline
\end{tabular}

Figure B-8. Schematic profile of hydrostratigraphic units in the Chestnut Ridge Hydrogeologic Regime. 


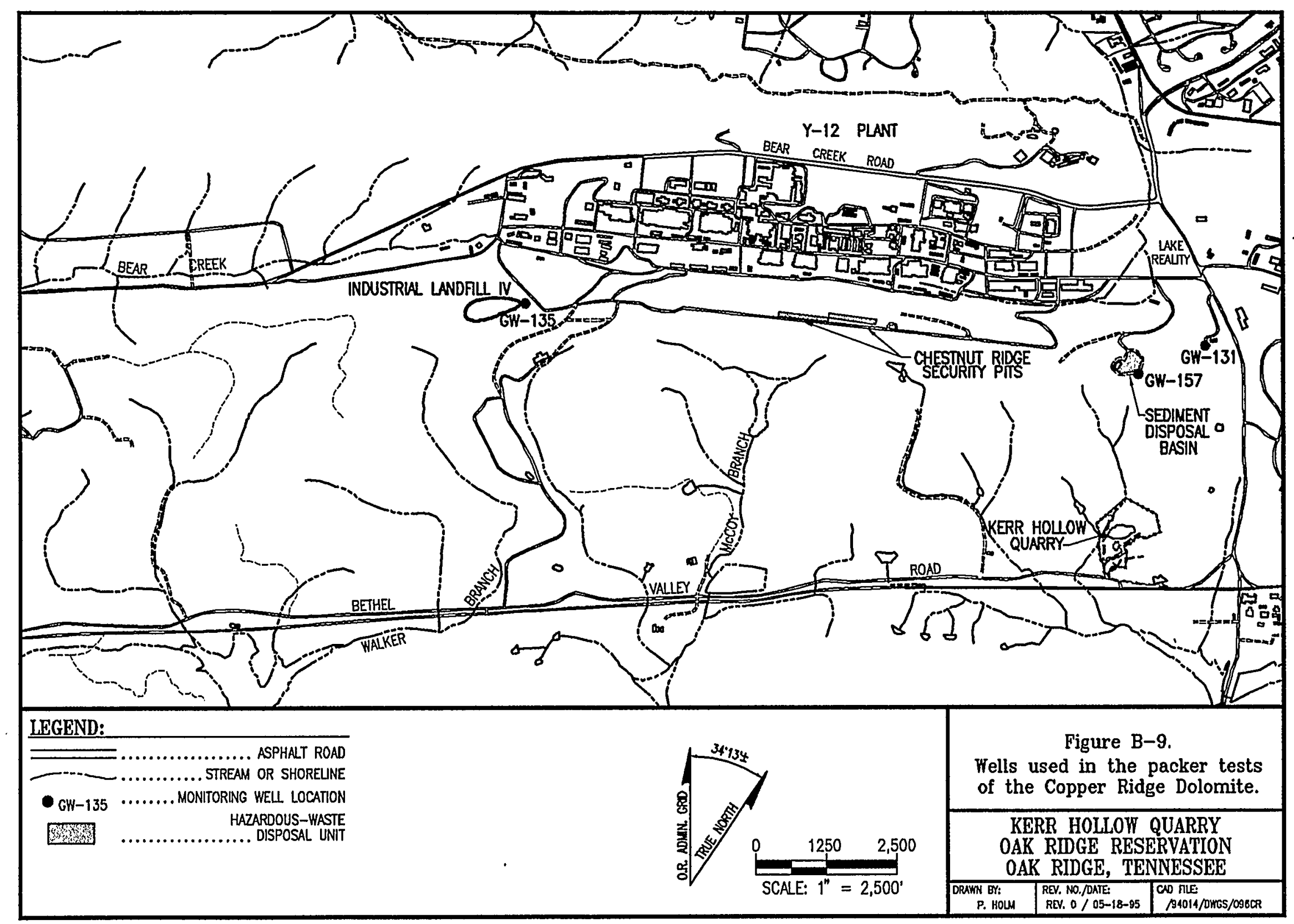




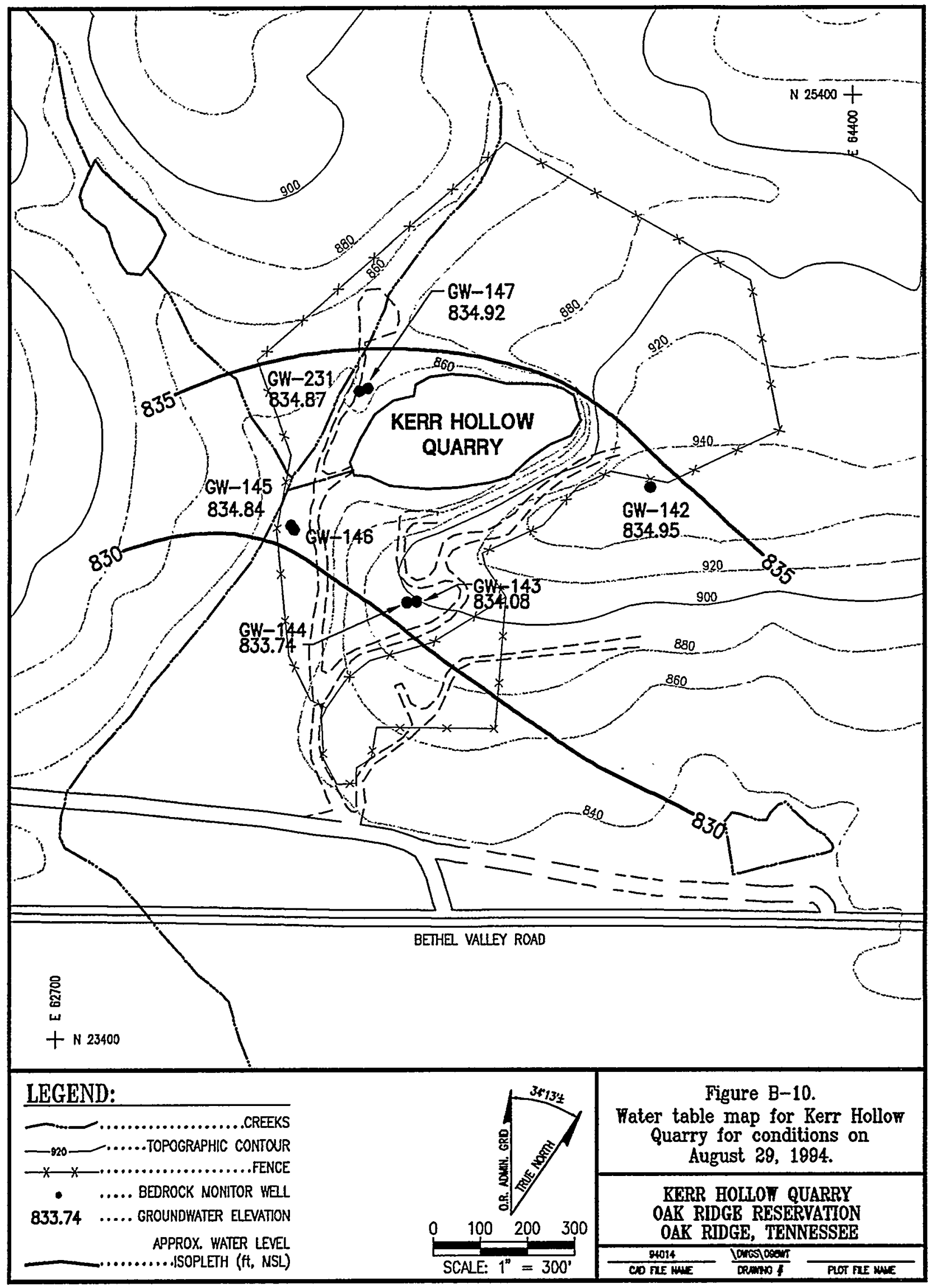




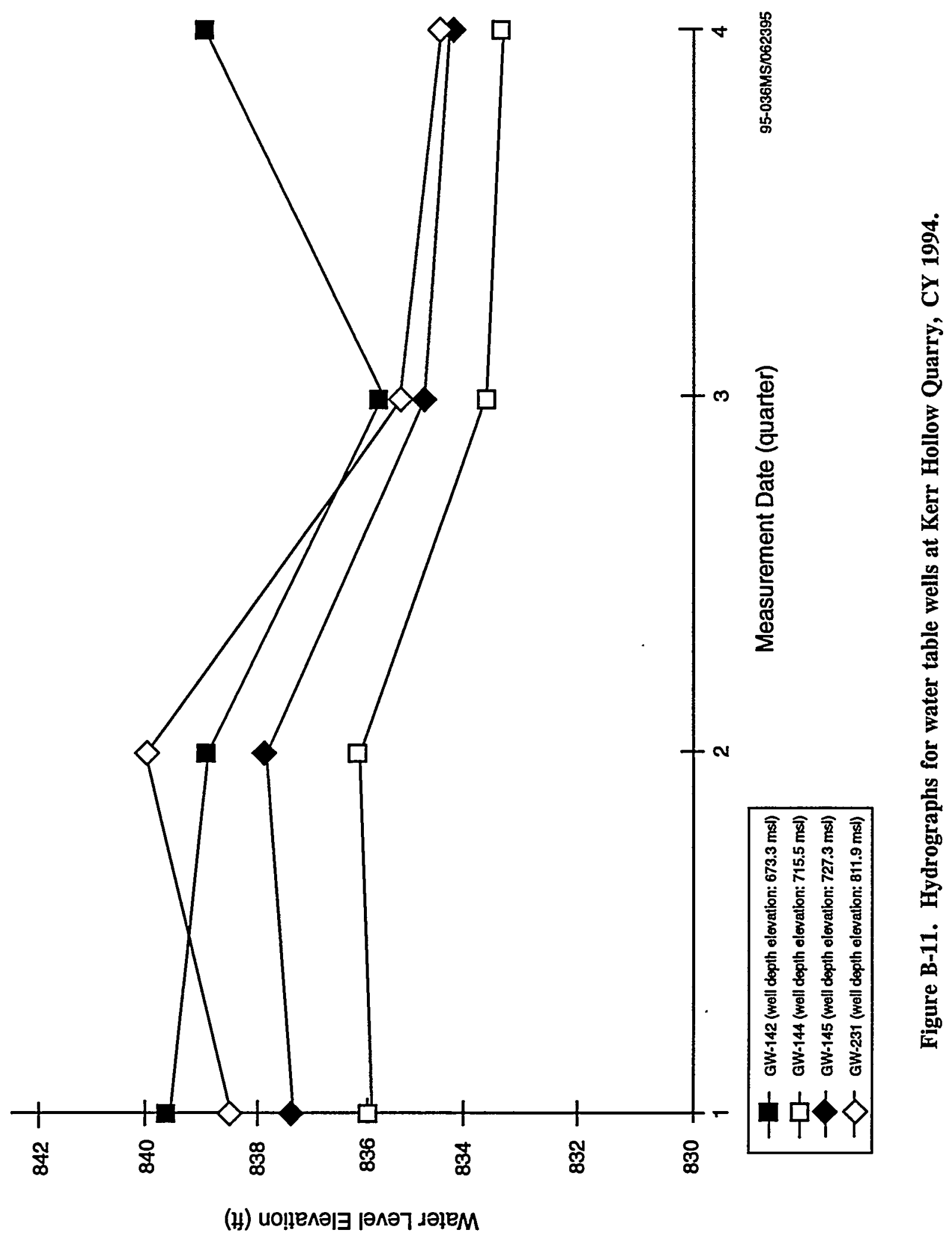




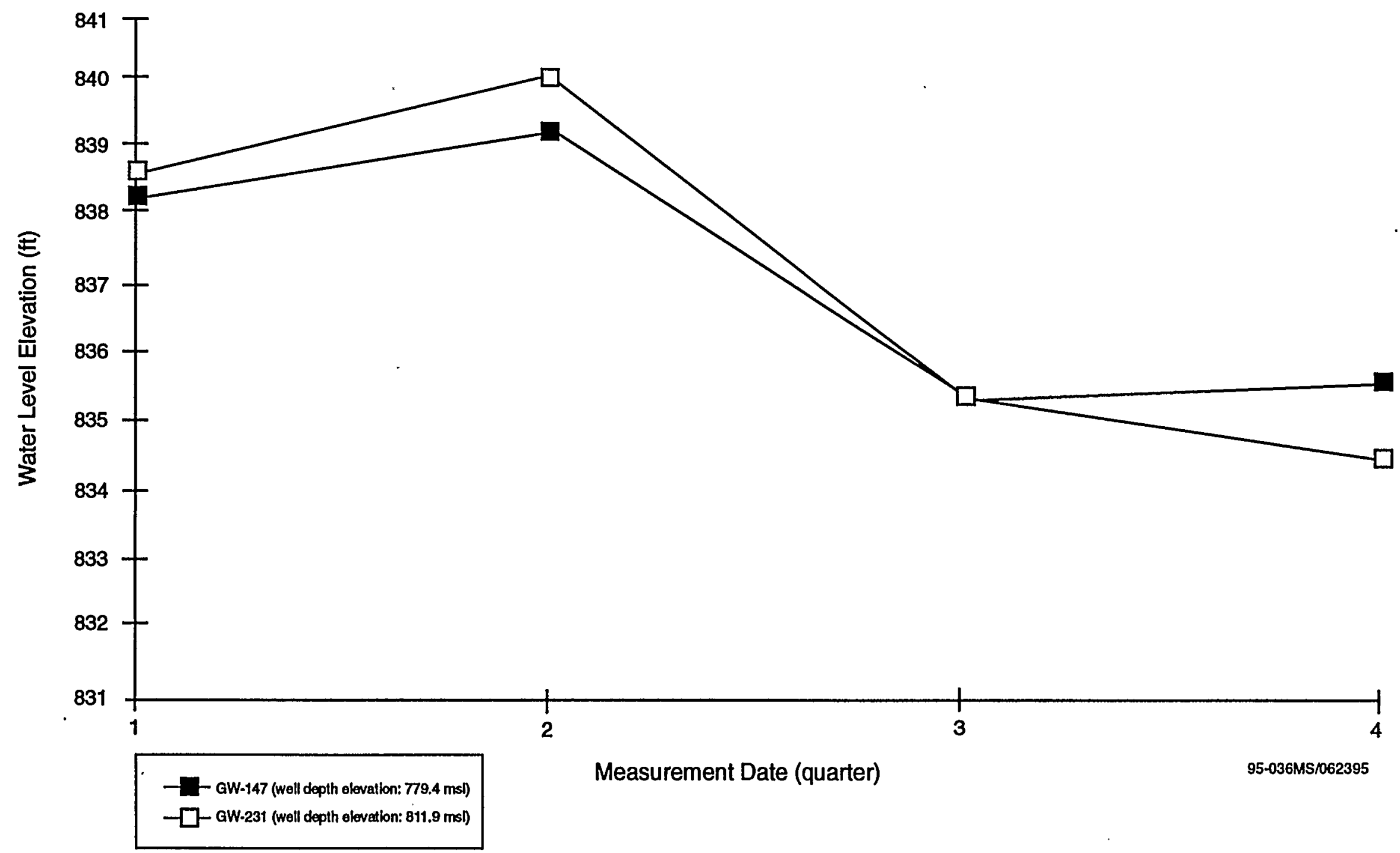

Figure B-12. Hydrographs for Wells GW-147 and GW-231, northwestern well cluster at Kerr Hollow Quarry, CY 1994. 


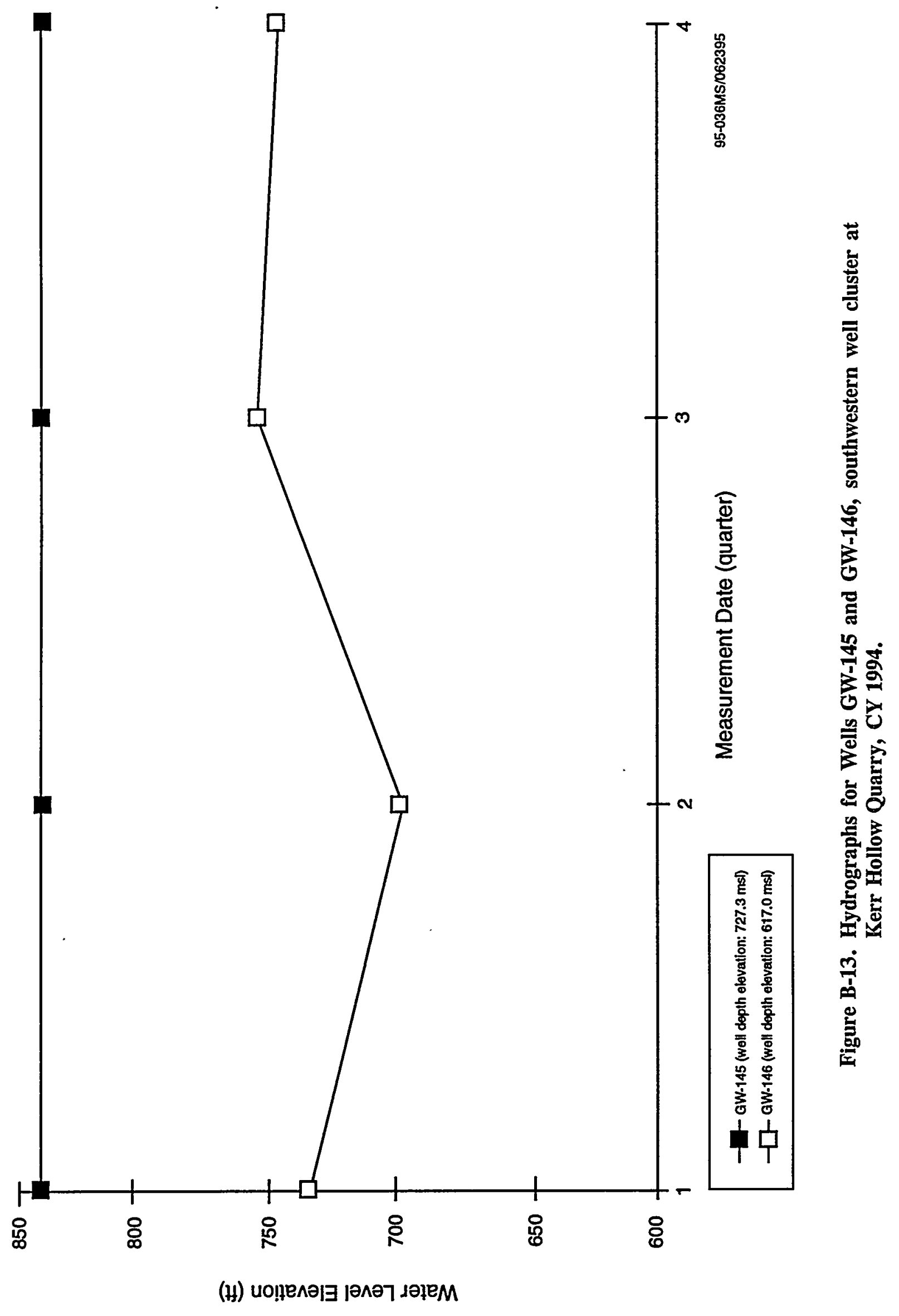




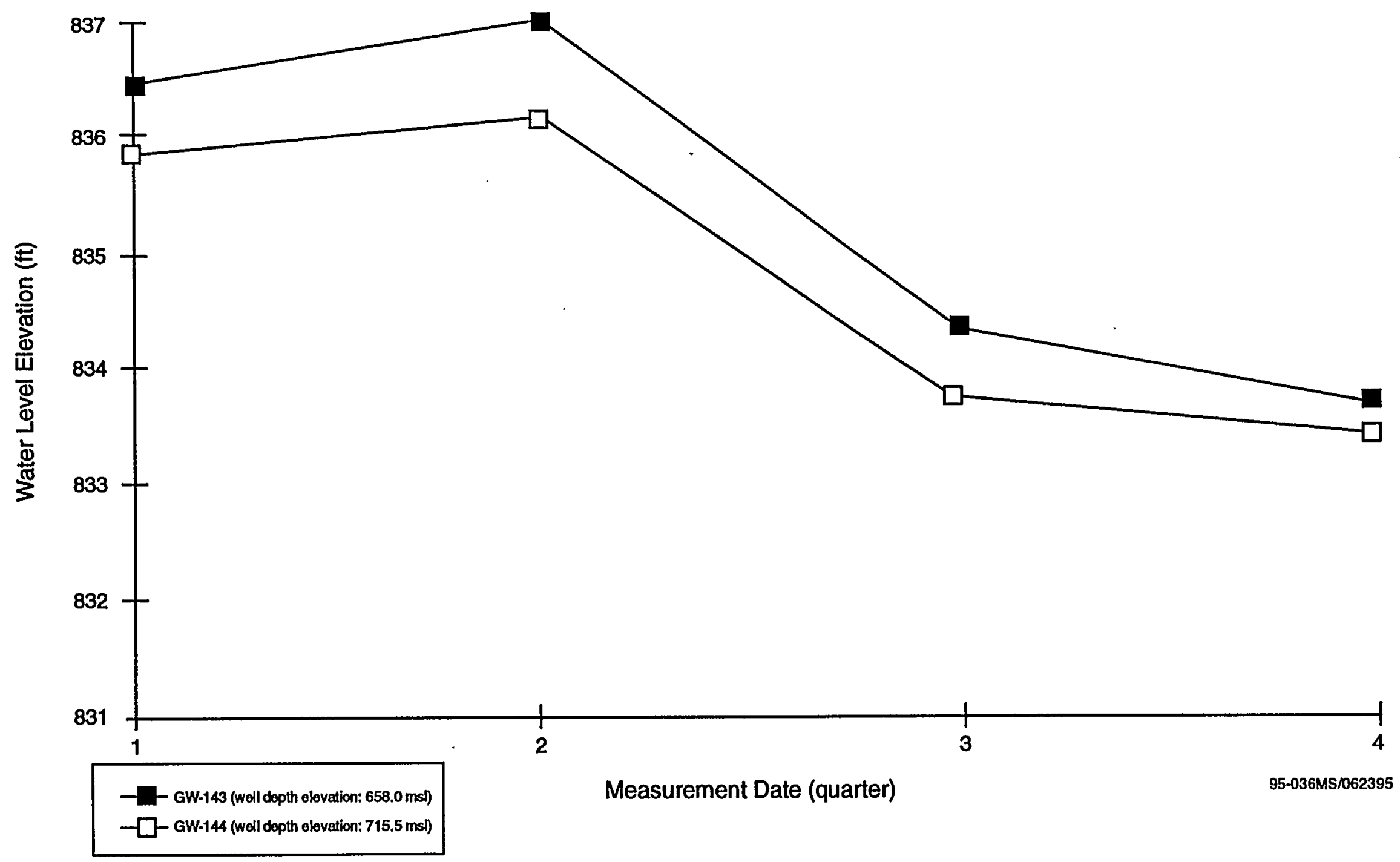

Figure B-14. Hydrographs for Wells GW-143 and GW-144, southern well cluster at Kerr Hollow Quarry, CY 1994. 


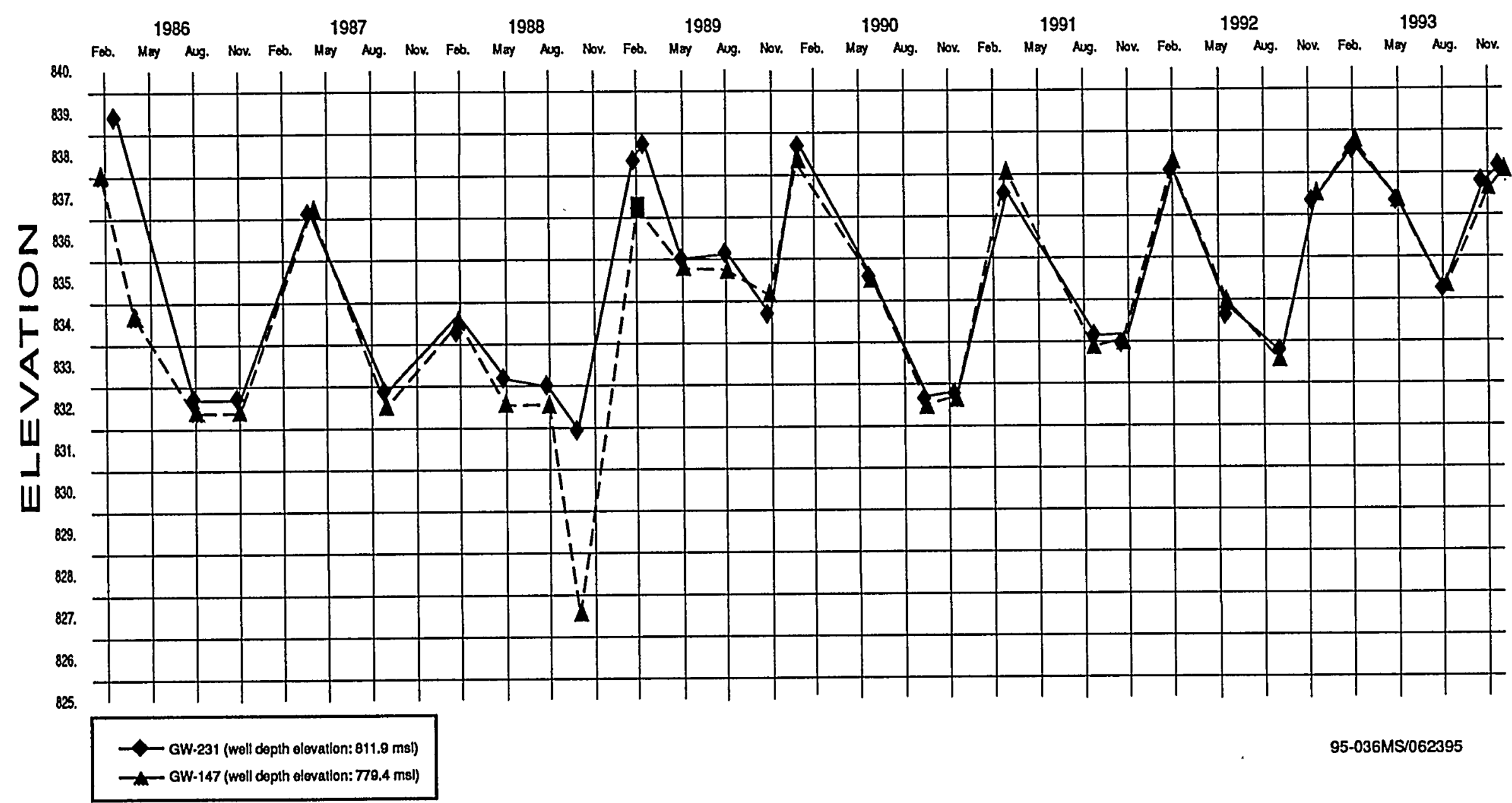

Figure B-15. Hydrographs for Wells GW-147 and GW-231, northwestern well cluster at Kerr Hollow Quarry, CY 1986 through CY 1993. 


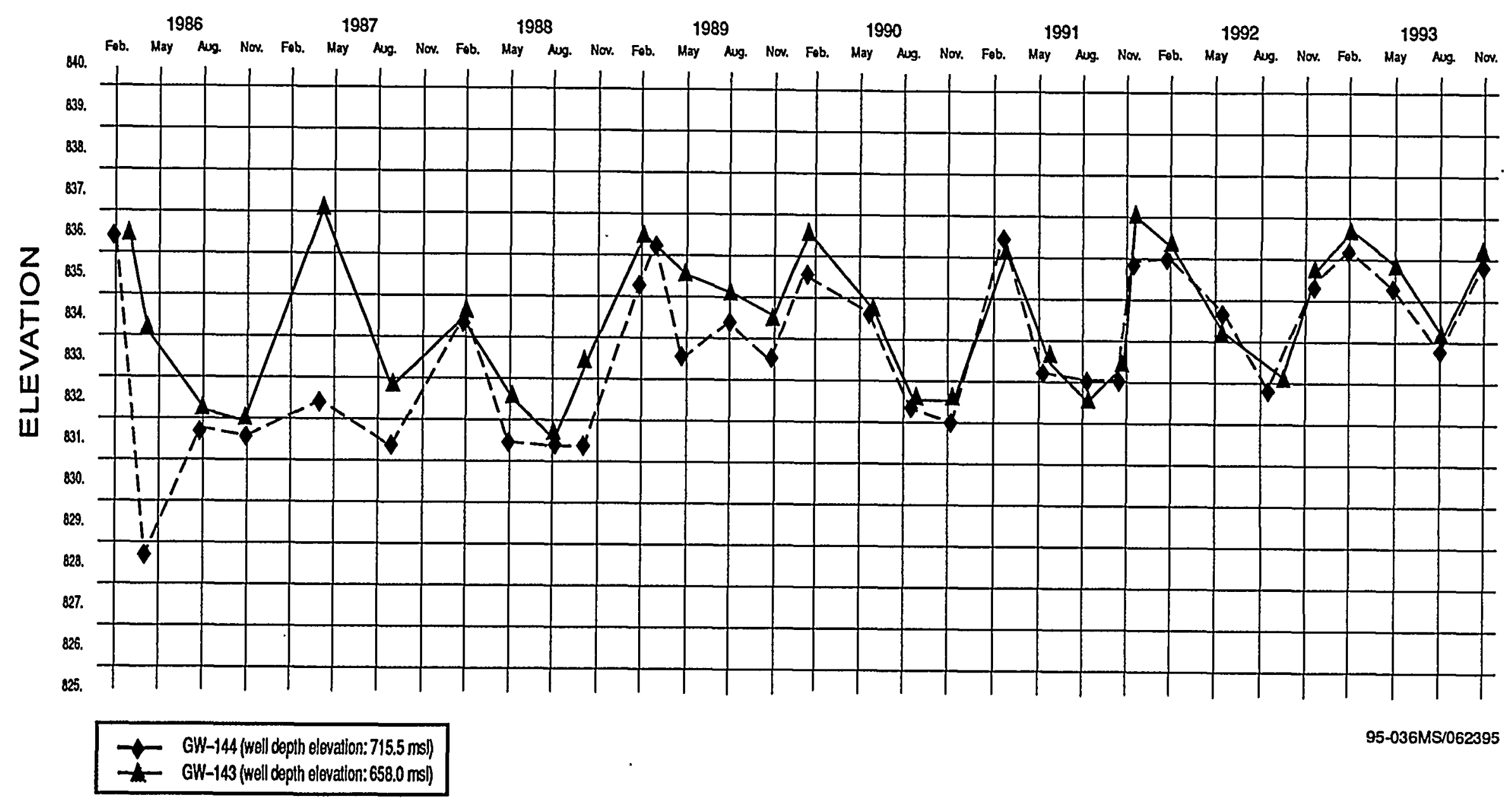

Figure B-17. Hydrographs for Wells GW-143 and GW-144, southern well cluster at Kerr Hollow Quarry, CY 1986 through CY 1993. 


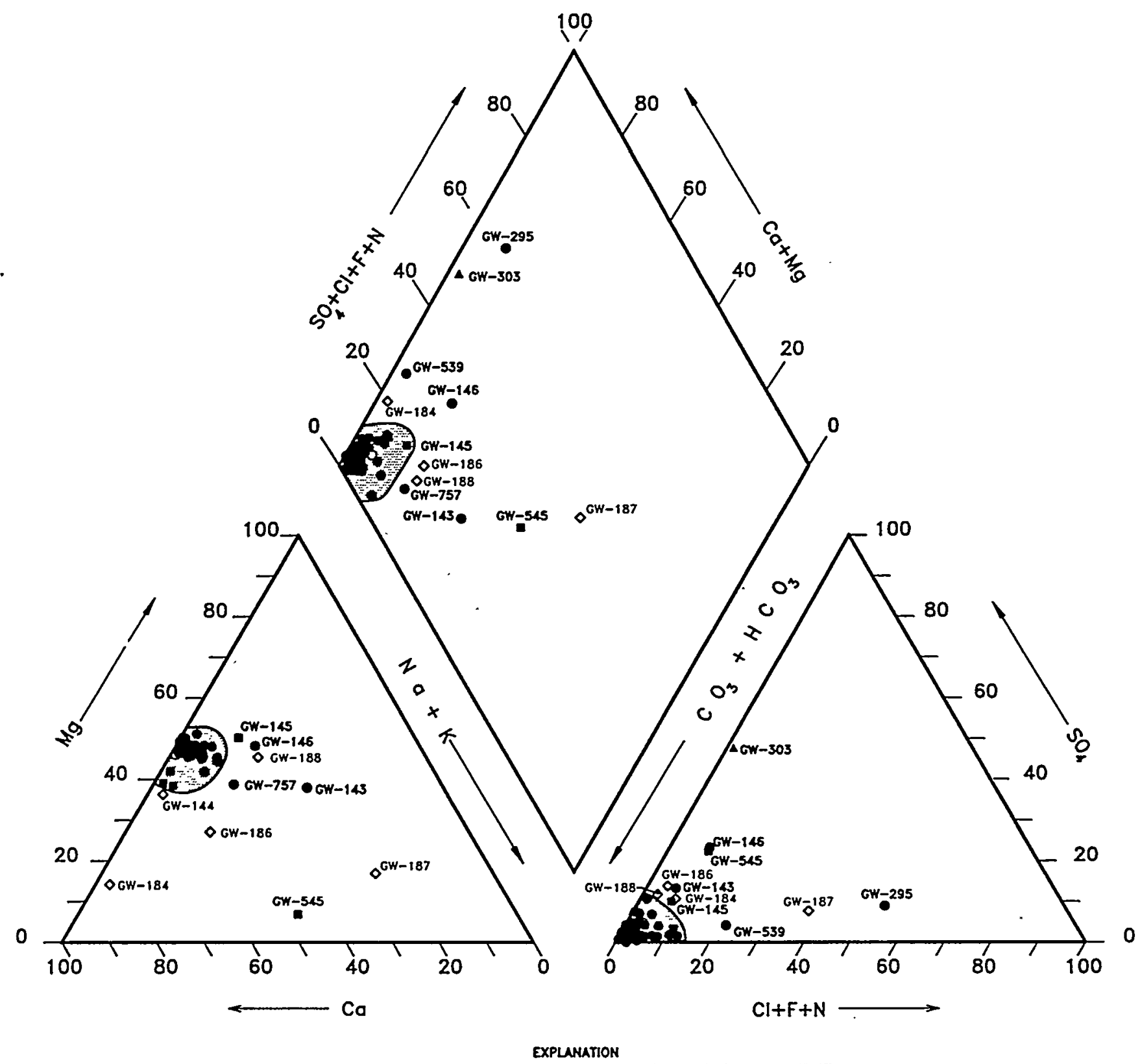

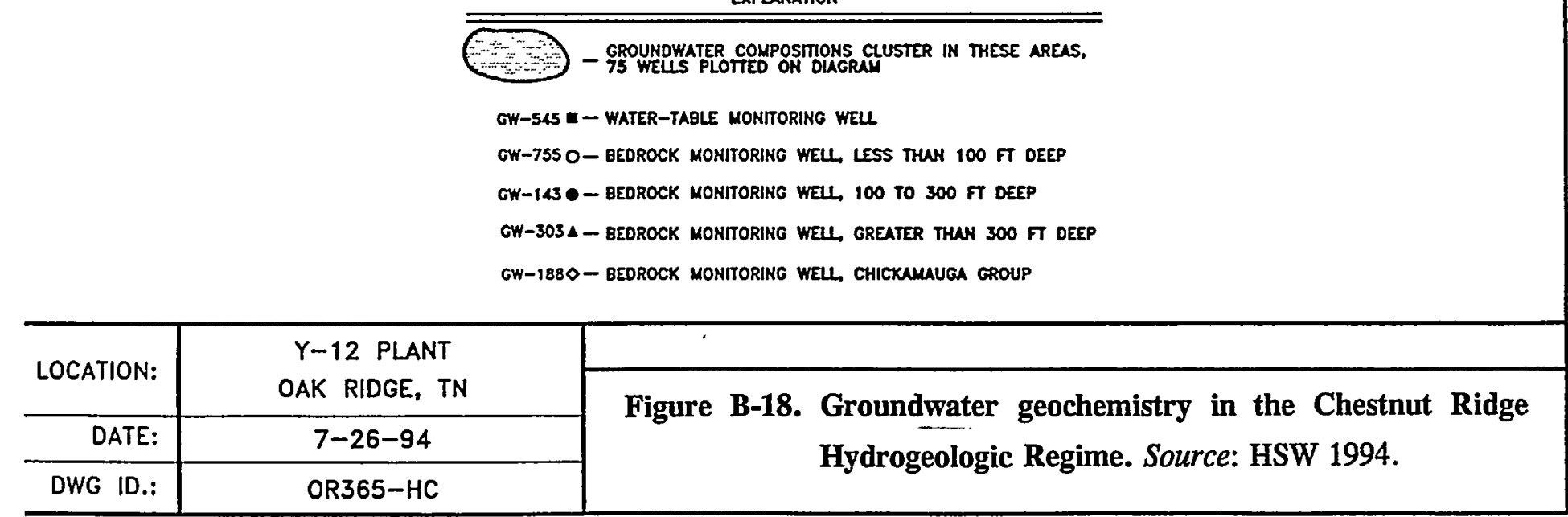




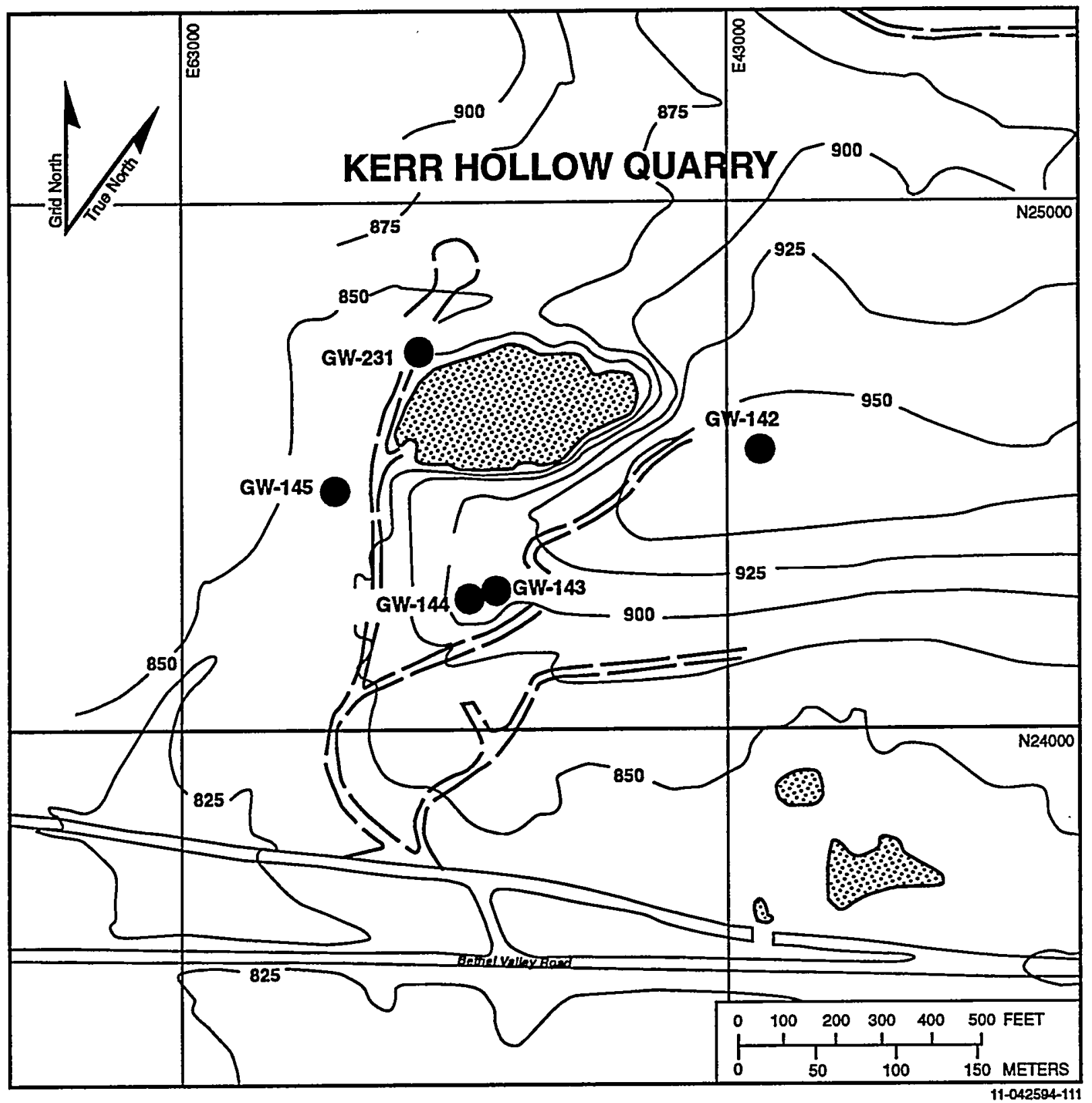

Figure B-19. Location of proposed groundwater monitoring well network at Kerr Hollow Quarry. 


\section{APPENDIX A}

RCRA CLOSURE/POST-CLOSURE PLAN FOR THE KERR HOLLOW QUARRY

A.1 REVISED CLOSURE PLAN FOR KERR HOLLOW QUARRY AT THE OAK RIDGE Y-12 PLANT, OAK RIDGE, TENNESSEE

\section{A.2 KERR HOLLOW QUARRY POST-CLOSURE PLAN}

\section{A.3 CLOSURE CERTIFICATION LETTER}




\section{A.1 REVISED CLOSURE PLAN FOR KERR HOLLOW QUARRY}

AT THE OAK RIDGE Y-12 PLANT, OAK RIDGE, TENNESSEE 
DOE/OR/01-1103\&D3
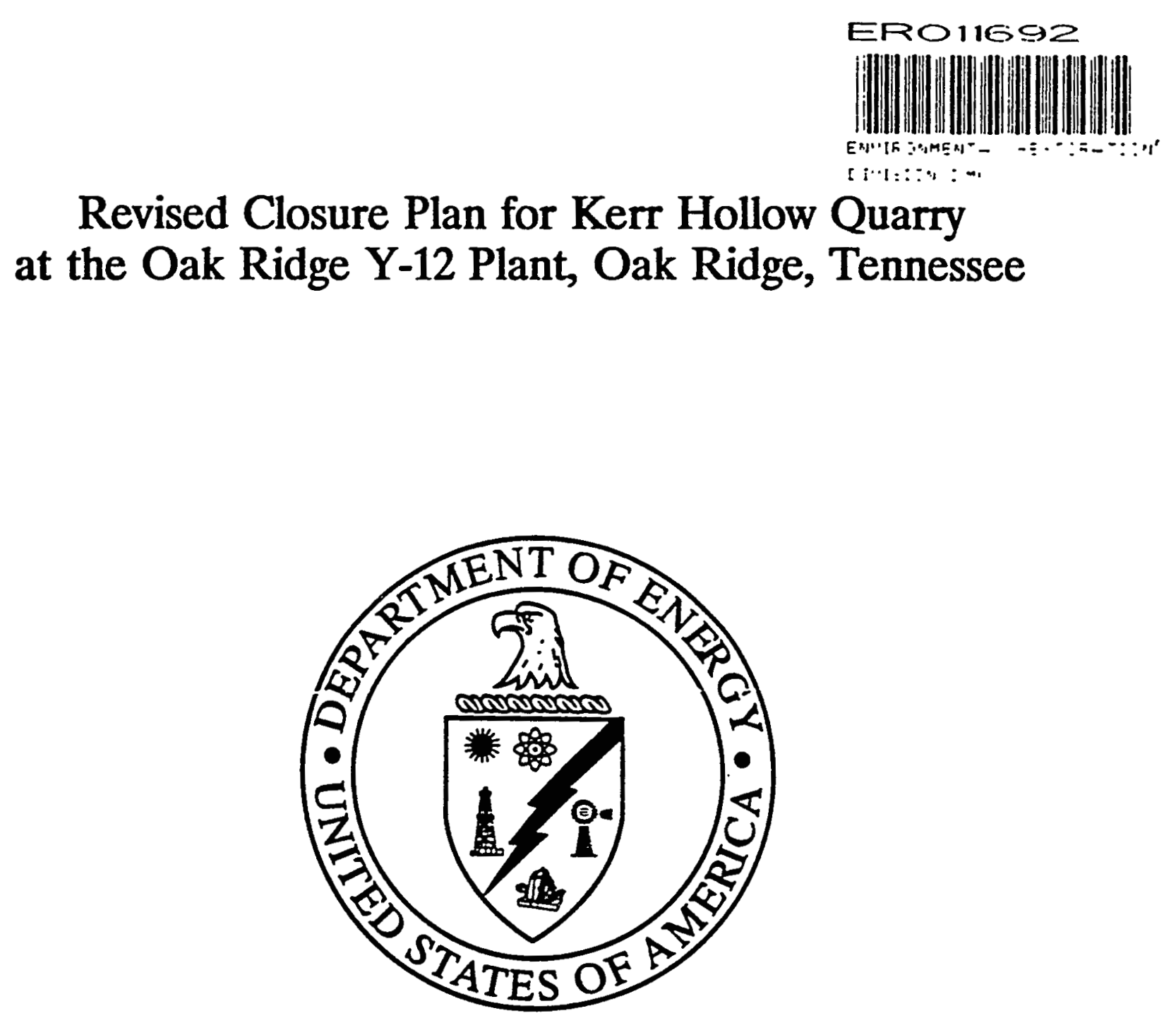
Energy Systems Environmental Restoration Program

Y-12 Environmental Restoration Program

Revised Closure Plan for Kerr Hollow Quarry at the Oak Ridge Y-12 Plant, Oak Ridge, Tennessee

Date Issued-April 1993

Prepared for

U.S. Department of Energy

Office of Environmental Restoration and Waste Management

under budget and reporting code EW 20

Prepared by the

OAK RIDGE Y-12 PLANT

managed by

MARTIN MARIETTA ENERGY SYSTEMS, INC.

for the

U.S. DEPARTMENT OF ENERGY

under contract DE-AC05-84OR21400 


\section{CONTENTS}

INTRODUCTION $\ldots \ldots \ldots \ldots \ldots \ldots \ldots \ldots \ldots \ldots \ldots \ldots \ldots \ldots \ldots \ldots \ldots \ldots$

PAST TREATMENT PRACTICES $\ldots \ldots \ldots \ldots \ldots \ldots \ldots \ldots \ldots \ldots \ldots \ldots \ldots$

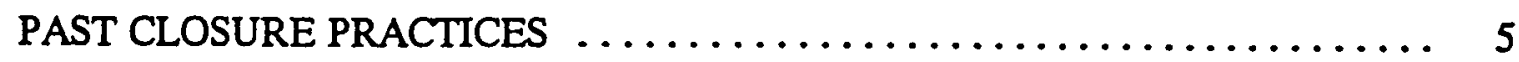

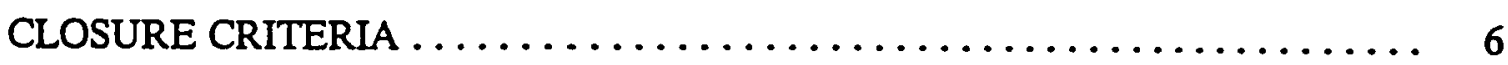

WATER AND MONITORING WELL SAMPLING AND RESULTS $\ldots \ldots \ldots \ldots \quad 7$

CLOSURE SCHEDULE $\ldots \ldots \ldots \ldots \ldots \ldots \ldots \ldots \ldots \ldots \ldots \ldots \ldots \ldots, 8$

MAINTENANCE OF SITE $\ldots \ldots \ldots \ldots \ldots \ldots \ldots \ldots \ldots \ldots \ldots \ldots, 8$

CERTIFICATION OF CLOSURE $\ldots \ldots \ldots \ldots \ldots \ldots \ldots \ldots \ldots \ldots \ldots \ldots$

NOTICE OF LOCAL LAND AUTHORITY $\ldots \ldots \ldots \ldots \ldots \ldots \ldots \ldots \ldots, 10$

RECORD OF WASTES $\ldots \ldots \ldots \ldots \ldots \ldots \ldots \ldots \ldots \ldots \ldots \ldots \ldots \ldots \ldots \ldots \ldots \ldots \ldots$

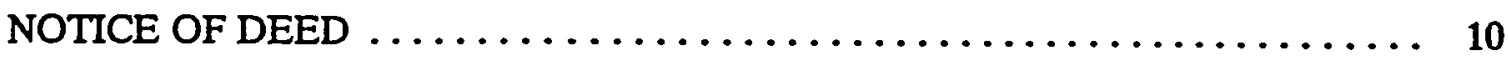

CERTIFICATION OF NOTICE $\ldots \ldots \ldots \ldots \ldots \ldots \ldots \ldots \ldots \ldots \ldots \ldots, 10$

REFERENCES $\ldots \ldots \ldots \ldots \ldots \ldots \ldots \ldots \ldots \ldots \ldots \ldots \ldots \ldots \ldots \ldots \ldots \ldots$ 


\section{INTRODUCTION}

Kerr Hollow Quarry (KHQ) is a rock quarry which was operated in the 1940s as a rock and gravel quarry but was abandoned in the late 1940s when the quarry filled with water. Since at least 1951, the Department of Energy's (DOE's) Oak Ridge Y-12 Plant and the Oak Ridge National Laboratory have used $\mathrm{KHQ}$ for the treatment of water-reactive materials, potentially explosive chemicals, and empty compressed gas cylinders. Wastes received at $\mathrm{KHO}$ were defined as hazardous wastes only by the characteristics of reactivity, corrosivity, or ignitability. Treatment of these reactive, corrosive, and ignitable wastes at KHQ effectively eliminated the hazardous characteristics of the wastes. The KHQ facility was not intended for use as a hazardous waste storage or disposal facility. KHQ was used strictly for the emergency handling of these materials when personnel safety was the primary concern. Access to the quarry is limited by an existing 7-ft-high chain-link barbed-wire-topped fence, and the entrance gates are kept locked at all times except for authorized personnel entry and exit. The location of KHQ in relation to the Y-12 Plant is shown in Fig. 1.

KHQ was a permitted facility under the National Pollutant Discharge Elimination System (NPDES), Permit No. TN 0002968-Outfall 301, for the treatment of certain potentially explosive chemicals or water reactive metals. The permit required monitoring the KHQ outfall following a treatment operation, with results reported quarterly to the Tennessee Department of Environment and Conservation (TDEC). Treatment operations at KHQ were not permitted when there was a visible surface discharge at the outfall.

\section{PAST TREATMENT PRACTICES}

Because of the hazardous/explosive nature of the materials treated at $\mathrm{KHQ}$, a metal building and chute were constructed on the southern bluff of the quarry, high above the water surface (see Fig. 2). The chute allowed the materials to be deposited directly into the water in the quarry without striking the bank. A separate cleared area exists on the northwest bank of the quarry where compressed-gas cylinders with frozen or leaky valves could be placed to allow penetration by rifle fire to vent the gases. The Y-12 security guards, who shot the containers floating in the quarry or the gas cylinders on the bank of the quarry, were positioned in the metal building on the southern bluff to protect them when the waterreactive or other materials exploded in the quarry.

Water-reactive metals such as lithium, potassium, calcium, sodium, or sodium-potassium alloy were normally packaged in 5-, 30-, or 55-gal containers and transported to the quarry, where they were placed in the chute and dropped to the water surface. These containers were punctured with rifle fire allowing water to contact the reactive metal or alloy. Water reacted with the metals, releasing hydrogen gas and forming oxides and hydroxides with the alkaline metal. Complete reaction of the metals is ensured because of the violent nature of the reactions; an explosion or violent burning was witnessed shortly after the container was punctured as the container sank below the surface of the water. A limited number of 500-gal 


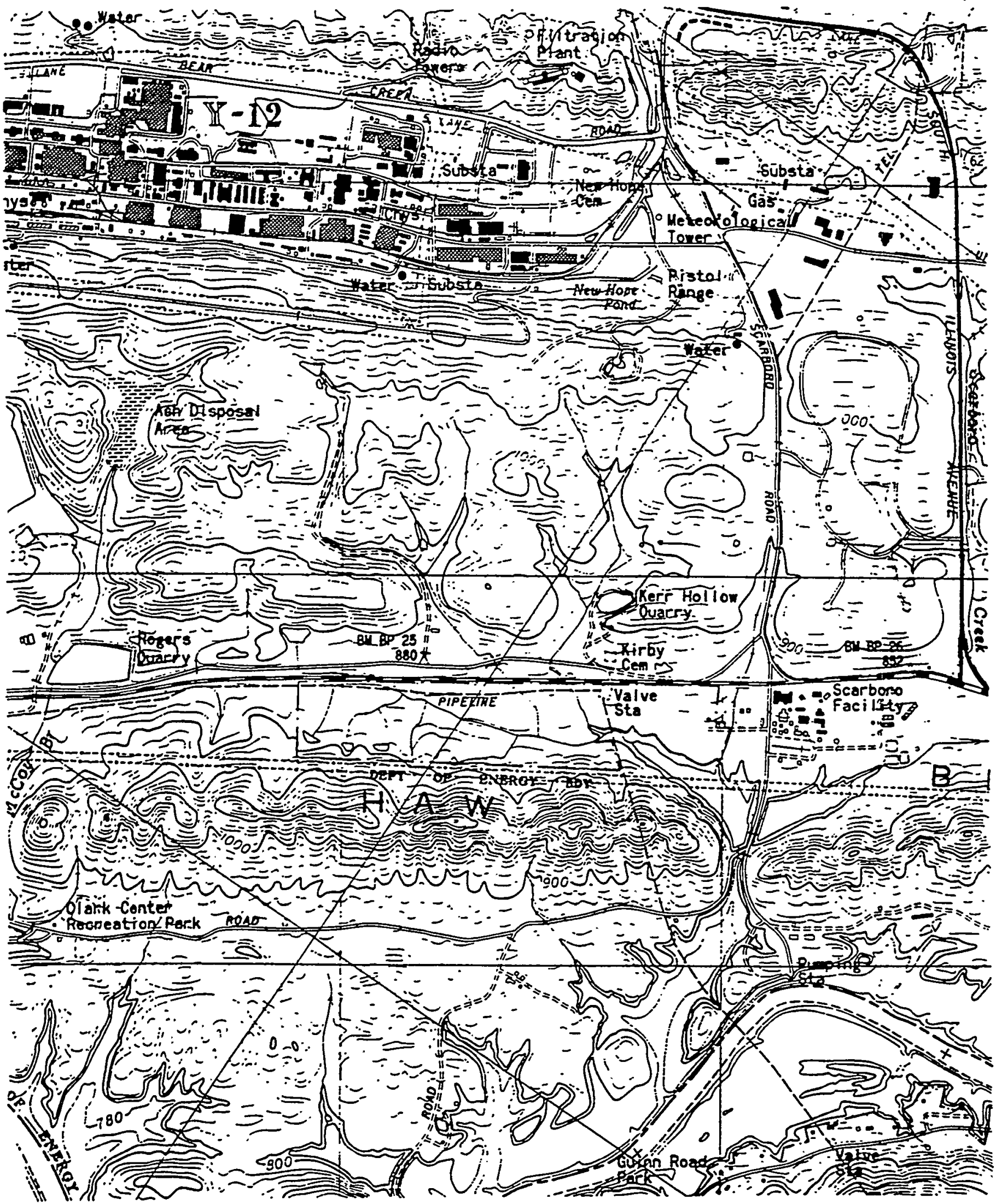

Fig 1. Topographic map showing the Oak Ridge Y-12 Plant, pertinent facilities, and surrounding area. 


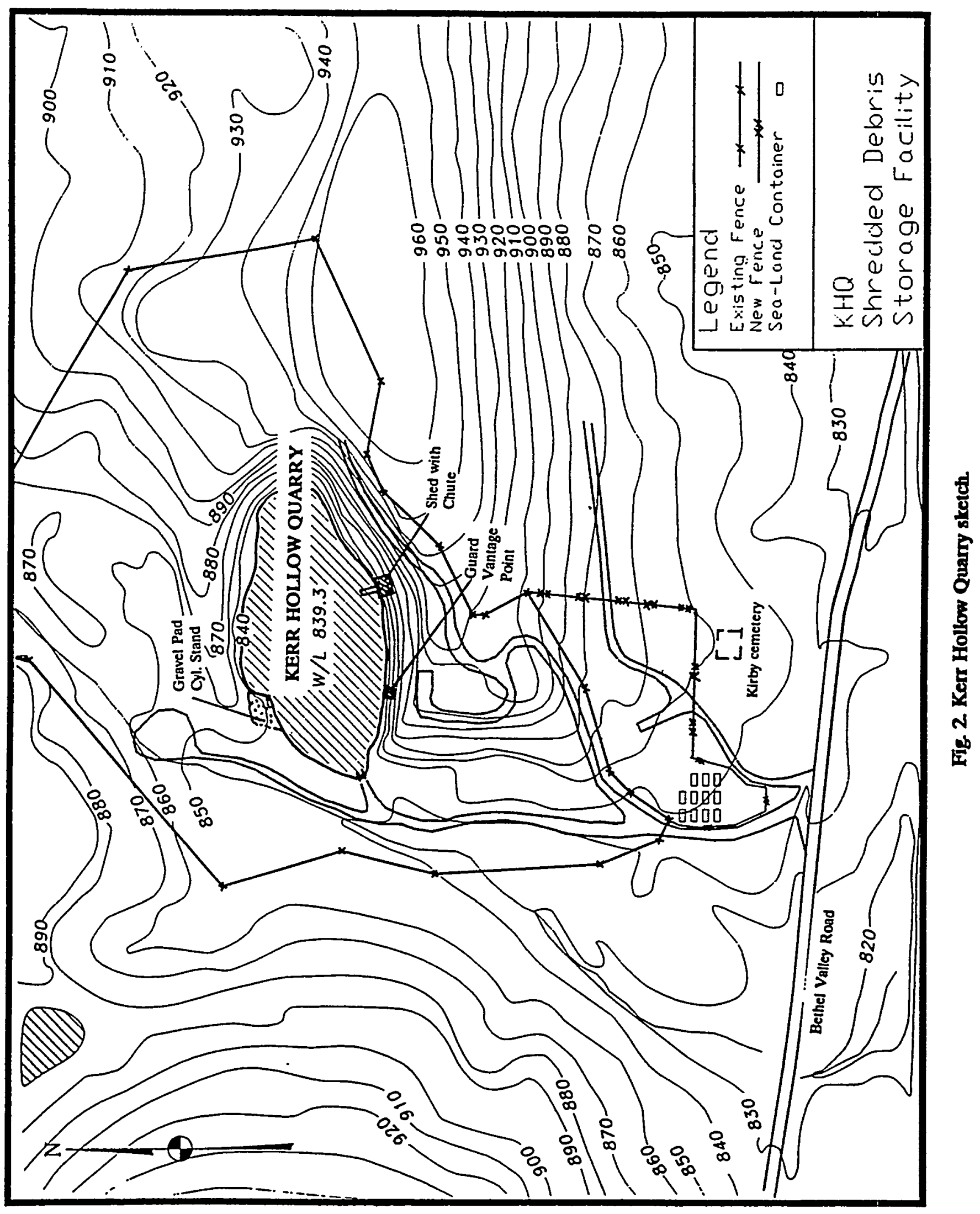


pressure vessels containing sodium were placed in the quarry. Those large containers, which were too heavy to float, were dropped from the upper rim of the quarry and allowed to vent as they entered the water by positioning valves and vent pipes in the open position.

Potentially explosive chemicals such as picric acid were transported to the quarry in specially packed containers to minimize shock and possible detonation in transit. The containers were suspended on a cable above the water surface and punctured with rifle fire prior to being released into the water.

Gas cylinders with frozen valves were vented on the northwest bank of the quarry by puncturing the cylinder wall with rifle fire. Once vented, the empty cylinders were removed from the area and returned to the Y-12 Plant for disposal; prior to the early 1970s, however, cylinders were discarded in the quarry. A typical listing of the materials that have been reacted or vented in the quarry is included in Table 1 . A detailed inventory of the volumes and types of materials to KHQ in past years is on file and available for review at the $\mathrm{Y}-12$ Plant.

Table 1. Description of typical wastes treated in Kerr Hollow Quarry

\begin{tabular}{ll}
\hline \multicolumn{1}{c}{ Waste type } & \multicolumn{1}{c}{ Examples } \\
\hline Alkali metals and metal hydrides & Lithium and lithium hydride \\
(water reactive) & Sodium and sodium hydride \\
& Potassium and potassium hydride \\
Calcium and calcium hydride & Zirconium hydride \\
& Titanium hydride \\
Lithium hydride \\
& Lithium aluminum hydride \\
& Sodium-potassium (NaK) metal \\
& Picric acid and related compounds \\
Unstable organics (explosive) & Ethers \\
& Peroxides \\
& Hydrazine \\
Metals (reactive) & Phosphorous \\
& Magnesium \\
Mechanical hazard (explosive) & Stressed steel parts (shrapnel danger) \\
& Vacuum tubes (implosive) \\
& Blasting caps \\
& Gas cylinders (with frozen/broken valves) \\
& Ammonia (irritant) \\
Miscellaneous hazards & Inorganic acids (corrosive) \\
& 50 tons \\
\hline
\end{tabular}

-The estimated total amount includes hazardous and nonhazardous materials that have been sent to KHQ since 1962 , when records were first kept. This estimated amount generally includes the weight of the containers and the materials that were sent to the quarry for treatment. 


\section{PAST CLOSURE PRACTICES}

In March 1989, a remote-operated underwater vehicle (ROV) equipped with a camera was used to survey the bottom of the quarry. A review of the videotapes showed that most of the drums accessible to the camera appeared to be empty of any residual reactive or explosive materials. Since records of past disposals show that in addition to the containers observed in the videotapes, numerous large steel vessels of reactive metals were treated and left in $\mathrm{KHQ}$, additional video surveys were made of $\mathrm{KHQ}$ to locate the large vessels. These large-vessel surveys were conducted during the spring and early summer months of 1991.

Also, because of the need at Y-12 for a Safety Assessment when dealing with potentially unsafe operations, a Safety Assessment was conducted by Y-12 during late 1988 and early 1989. The Safety Assessment and subsequent safety reviews evaluated whether all or part of the containers in KHQ could be moved and breached safely while ensuring the protection of human health and the environment. The planned closure activities were assessed as safe, and they are discussed in the following paragraphs.

To ensure that the large vessels on the quarry's bottom are empty, a remote method of breaching suspect containers will be used to confirm that no reactive or explosive wastes remain. The remote method of breaching will consist of placing explosive charge(s) on the drums or steel vessels, detonating the charge(s), and viewing the ruptured vessels by a remotecontrolled camera attached to an ROV. After the breached containers are inspected and appear empty, the containers will be removed.

Gas cylinders will be breached underwater using hydraulically powered equipment and then removed from the quarry.

Other containers (5-gal buckets, 30- and 55-gal barrels, and other miscellaneous items) will be shredded underwater. This process will provide the greatest assurance that all reactive materials have been exposed to water. The shredded material will be removed from the quarry.

Obviously empty items will be inspected and then removed from the quarry as nonhazardous waste. All visible items/material removed from the quarry will be inspected as necessary to ensure proper disposal.

In accordance with a state of Tennessee (TDEC) request under the Tennessee Water Quality Control Act (letter dated October 11, 1987), disposal operations at KHQ were terminated on November 7,1988, and steps were initiated to close the quarry in a manner adequate to meet closure requirements of a surface impoundment as specified in 40 CFR 265 . 


\section{CLOSURE CRITERIA}

As outlined in the previous sections, substantial progress has been made in eliminating the residual hazardous material present in the quarry, thereby minimizing to the extent necessary to protect human health and the environment the postclosure escape of hazardous waste or hazardous constituents to the groundwater or surface water or to the atmosphere. Visible containers or structures will be removed from the quarry after they are inspected. Closed containers, possibly containing reactive/hazardous material, will undergo shredding or breaching underwater to promote deactivation of reactive material.

The shredded debris is a residue of a primary treatment process designed to deactivate the reactive constituents. This treatment residue will be transported as nonhazardous waste to the Y-12 Plant and dispositioned to the Walk-In Pits at Bear Creek Burial Ground.

During closure activities at KHQ, visible containers will be breached to ensure that no waste material remains in the quarry. The material in the containers is water-reactive waste and is rendered nonhazardous after the chemical reaction has been concluded. If the final inspection of a breached container reveals that the entire contents have not been reacted, subsequent breaching efforts will be performed to ensure that no waste remains. If an unidentified material is found, a field evaluation will be performed to ensure that the treatment method protects human health and the environment.

Final inspection videotapes will be made of the quarry bottom to document that all visible containers have been removed. The videotapes will serve as verification that the closure requirements have been met.

- Y-12 will continue to sample the groundwater wells around the quarry until closure activities are complete and closure has been certified as complete. This action is intended to confirm that if any containers are left in the quarry, they are not causing the groundwater at KHQ to exceed the TDEC groundwater quality standards.

The sediment in the quarry and any items/containers that may be concealed beneath the sediment will remain in the quarry. Should further investigation or remediation of the quarry be required, those activities will be conducted under the authority of the existing ORR Federal Facility Agreement between DOE, EPA, and TDEC. Subsequent investigations or other pertinent environmental monitoring activities will be conducted by the ER Program as part of the ongoing CERCLA activities at the Y-12 Plant. These actions will be conducted (if required) in lieu of the requirements found in 40 CFR 265.228b and Tennessee Rule 12001-11-.05-112. Actions necessary under CERCLA will most likely include posting the KHQ site, maintaining a security fence, conducting regular security inspections, monitoring groundwater, and monitoring under the NPDES permit. 


\section{WATER AND MONITORING WELL SAMPLING AND RESULTS}

Kerr Hollow Quarry has an NPDES permit, which requires monitoring at the KHQ outfall within 24 hours after a treatment operation in the quarry and submittal of quarterly reports to TDEC if there has been a treatment operation during the previous month. Table 2 lists the parameters that must be analyzed to satisfy the NPDES permit, along with standards for those parameters, required monitoring frequency, and sample type. The results of previous years of sampling are available in numerous Y-12 documents (Geraghty and Miller 1985, Oakes et al. 1987) or in the quarterly monitoring reports that have been submitted to TDEC. In summary, the only times the NPDES permit conditions were exceeded were once in 1983 involving the upper $\mathrm{pH}$ limit and once in 1984 involving the lower $\mathrm{pH}$ limit. The discharge limits for other parameters, total suspended solids, potassium, lithium, sodium, heavy metals, etc., have not been exceeded at the KHQ outfall.

Table 2. NPDES emucnt limitations and monitoring requirements for discharges at Kerr Hollow Quarry

\begin{tabular}{|c|c|c|c|c|}
\hline \multirow[b]{2}{*}{ Effluent parameters } & \multicolumn{2}{|c|}{ Effluent limitations } & \multicolumn{2}{|c|}{ Monitoring requirements } \\
\hline & $\begin{array}{c}\text { Daily } \\
\text { average }\end{array}$ & $\begin{array}{l}\text { Daily } \\
\text { maximum }\end{array}$ & $\begin{array}{l}\text { Measurement } \\
\text { frequency }\end{array}$ & $\begin{array}{c}\text { Sample } \\
\text { type }\end{array}$ \\
\hline Flow, $\mathrm{m}^{3} / \mathrm{d}$ & & & Continuous & \\
\hline Total suspended solids, mg/L & 30.0 & 50.0 & $a$ & Grab \\
\hline Zirconium, mg/L & & 3.0 & $a$ & Grab \\
\hline Potassium, mg/L & & & $a$ & Grab \\
\hline Lithium, mg/L & & 5.0 & $a$ & Grab \\
\hline Sodium, mg/L & & & $a$ & Grab \\
\hline Heavy metals, mg/L & & & $a, b$ & Grab \\
\hline Temperature, ${ }^{\circ} \mathrm{F} /{ }^{\circ} \mathrm{C}$ & & $86.9 / 30.5$ & $a$ & Grab \\
\hline pH & & $6.5-8.5$ & After disposal & Grab \\
\hline
\end{tabular}


Seven groundwater wells were installed around the KHQ site, and an eighth boring is an exploratory core hole for subsurface characterization. The well locations are shown in Fig. 3. The seven wells at the KHQ site were all sampled during each of the four quarters of CY 1986. The sampling and analysis program being followed is consistent with state regulation TN 1200-1-11-.05, "Interim Status Groundwater Monitoring Requirements." The program is outlined as follows:

1986

Indicator Parameters

$\mathrm{pH}$

Specific conductance

Total organic carbon

Total organic halogen

$\begin{array}{cc}\text { Parameters Establishing Groundwater Quality } \\ \text { Chloride } & \text { Phenols } \\ \text { Iron } & \text { Sodium } \\ \text { Manganese } & \text { Sulfate }\end{array}$

1987-1989

Semiannually for indicator parameters

Annually for groundwater quality parameters and total uranium

\section{CLOSURE SCHEDULE}

A closure schedule is presented in Table 3.

Table 3. Kerr Hollow Quarry closure schedule

\begin{tabular}{lcc}
\hline \multicolumn{1}{c}{ Activity } & $\begin{array}{c}\text { Closure plan } \\
\text { dates }\end{array}$ & Actual dates \\
\hline $\begin{array}{l}\text { Review survey(s) information and map location of } \\
\text { containers in KHQ }\end{array}$ & $09 / 28 / 88$ & $09 / 28 / 88$ \\
Perform survey/final removal operations & & \\
Submit final closure certification to TDEC & $12 / 31 / 93$ & \\
\hline
\end{tabular}

\section{MAINTENANCE OF SITE}

The KHQ site will remain under DOE management for the perceivable future. The fence network, gates and locks, and posted signs will be inspected quarterly. If any damage is found, the missing fence, signs, etc., will be replaced. 


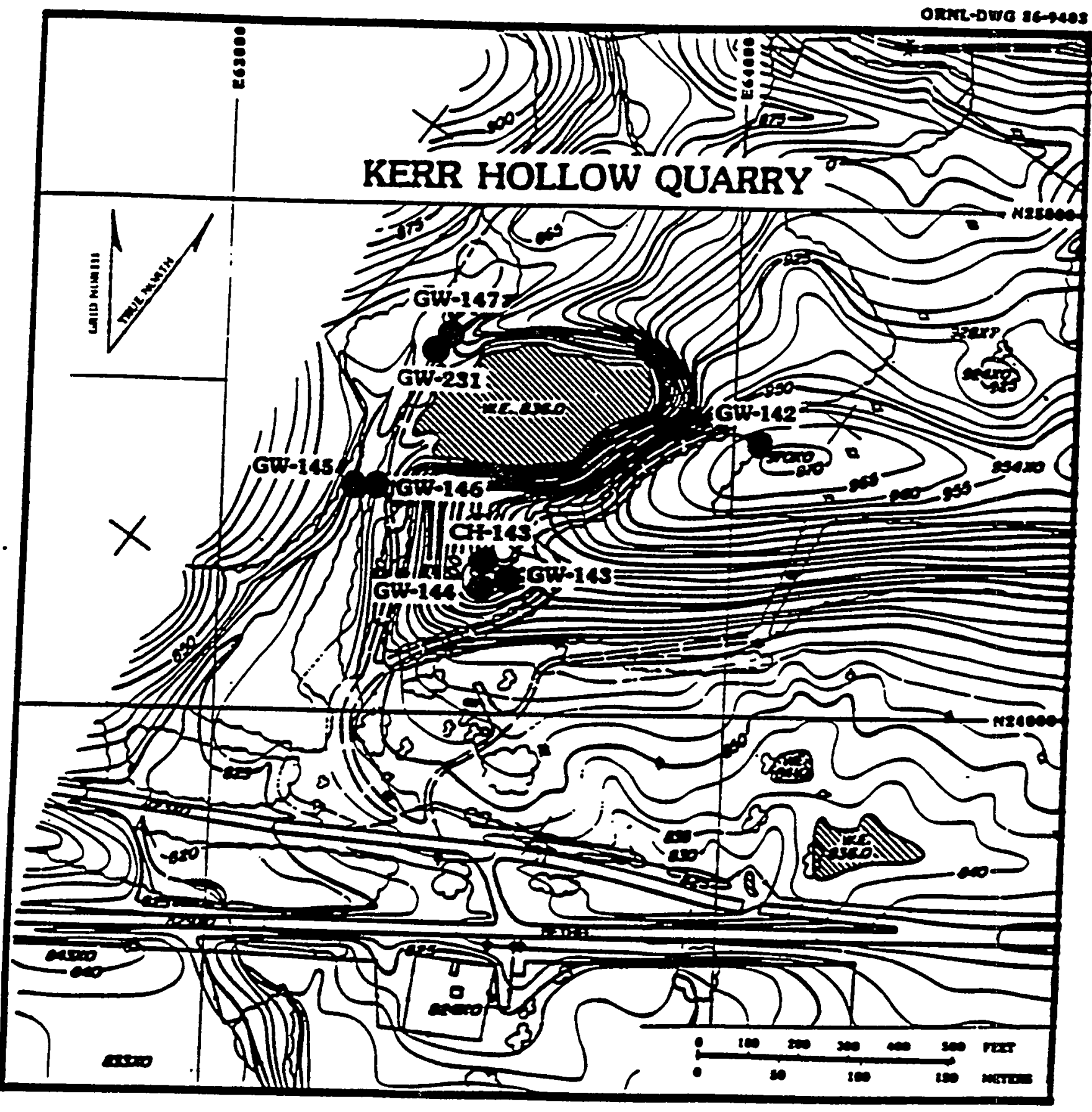

Fig 3. Location of monitoring welk. 


\section{CERTIFICATION OF CLOSURE}

DOE will submit a closure certification from both DOE and an independent registered professional engineer that closures have been completed in accordance with the approved closure plan. The certification shall be submitted to the Commissioner of TDEC within 60 days after completion of the last closure activity.

\section{NOTICE OF LOCAL LAND AUTHORITY}

Within 90 days after closure is completed, a survey plat indicating the location and dimensions of the KHQ will be submitted to the local land authority and to the Commissioner of TDEC. The plat will be prepared and certified by a professional land surveyor. The survey plat will contain the following prominently displayed note, or a similarly worded note:

The owner or operator of the property containing the Kerr Hollow Quarry, as shown on this plat, is obligated to restrict disturbance of the site in accordance with Rules Governing Hazardous Waste Management in Tennessee. Postclosure use of the property is limited to authorized personnel only, unless the owner or operator receives approval from the Commissioner of the Tennessee Department of Environment and Conservation under the previously referenced rules.

\section{RECORD OF WASTES}

A record of waste disposed of in $\mathrm{KHQ}$ and a record of removed waste will be submitted to the Commissioner of TDEC and the local land authority.

\section{NOTICE OF DEED}

The note presented in the section entitled Notice of Local Land Authority or a similarly worded note will be recorded on the facility property deed or on some instrument normally examined during a title search.

\section{CERTIFICATION OF NOTICE}

A certification by DOE will be submitted to the Commissioner of TDEC stating that the required notices have been filed. A copy of the document in which the notation has been placed will be attached. 


\section{REFERENCES}

Geraghty and Miller, Inc. 1985. Proposed Groundwater Monitoring Plans for the Kerr Hollow and the Rogers Quarries, Y/SUB-85-00206C/4R1, Martin Marietta Energy Systems, Inc., Oak Ridge Y-12 Plant, Oak Ridge, Tennessee, revised April 1986.

Oakes, T. W., C. W. Kimbrough, P. M. Pritz, S. T. Goodpasture, S. F. Huang, C. S. Gist, C. W. Weber, and F. M. O'Hara. 1987. Environmental Surveillance of the U.S. DOE Oak Ridge Reservation and Surrounding Environs During 1986, Volume 2: Data Presentation, ES/ESH-L/V2, Martin Marietta Energy Systems, Inc., Oak Ridge, Tennessee, April. 


\section{DISTRIBUTION}

1. E. T. Collins

2. M. F. P. DeLozier

3. J. D. Gass

4. C. D. Goins

5. J. T. Grumski

6. P. J. Halsey

7. J. E Heiskell

8. P. H. Hight

9. C. C. Hill

10. R. M. Hill

11. I. W. Jeter

12. W. D. Jones

13. A. K. Lee

14. L.W. Long

16-18. D. M. Matteo

19. L. S. McAdams

20. L. W. McMahon

21. J. Q. Miller

22. M. Mitchell

23. J. W. Moore
24. H. C. Newsom/D. C. White

25-26. P. T. Owen

27. T. P. A. Perry

28. J. E. Powell

29. G. E. Rymer

30. J. K Siberell

31. C. M. Smith

32. J. E. Stone

33. C. C. Travis

34. R. R. Turner

35. J. H. Vanderlan

36. K L Walker

37. R. W. Weigel

38. R. K White

39. A. C. Williamson

40. A. K. Zava

41. Central Research Library

42-46. ER Document Management Center

47. Y-12 ER Document Center

48. $Y-12$ Central Files

49. H. W. Hibbits, DOE Oak Ridge Field Office, P.O. Box 2001, Oak Ridge, TN 37831-8541

50-51. R. L. Nace, Branch Chief, Nonenrichment Facilities, Oak Ridge Program Division, Office of Eastern Area Programs, Office of Environmental Restoration, EM-423, Trevion 2, U.S. Department of Energy, Washington, DC 20585

52-58. S. L. Lankford, DOE Oak Ridge Field Office, P.O. Box 2001, Oak Ridge, TN 37831-8541

59. R. D. Oglesby, DOE Oak Ridge Field Office, P.O. Box 2001, Oak Ridge, TN 37831-8541

60. J. Powell, DOE Oak Ridge Field Office, P.O. Box 2001, Oak Ridge, TN 37831-8541

61. R. J. Spence, DOE Oak Ridge Field Office, P.O. Box 2001, Oak Ridge, TN 37831-8541

62. D. W. Swindle, Radian Corporation, 120 S. Jefferson Circle, Oak Ridge, TN 37830

63-64. H. M. Thron, Chief, Enrichment Facilities, Oak Ridge Program Division, Office of Eastern Area Programs, Office of Environmental Restoration, EM-423, Trevion 2, U.S. Department of Energy, Washington, DC 20585

65-66. Office of Scientific and Technical Information, P.O. Box 62, Oak Ridge, TN 37831 


\section{A.2 KERR HOLLOW QUARRY POST-CLOSURE PLAN}




\section{KERR HOLLOW QUARRY POST-CLOSURE PLAN}

\subsection{Post-Closure Care and Use of Property}

Ref: $\quad 1200-1-11-.06(7)$

40 CFR 264.117

This plan outlines the post-closure care activities to be conducted at the Kerr Hollow Quarry (KHQ). The property will be maintained in accordance with the post-closure plan upon approval by the Tennessee Department of Environment and Conservation (TDEC).

\subsection{Length of Post-Closure Care Period}

Ref: $\quad 1200-1-11-.06(7)$

40 CFR 264.117(a)(1)

Post-closure care activities at the KHQ will continue for 30 years after the date of closure.

\subsection{Increasing/Decreasing Length of Post-Closure Care Period}

Ref: $\quad 1200-1-11-.06(7)$

40 CFR 264.117(a)(2)

All activities described in this post-closure plan will be conducted for the duration of the post-closure care period unless a demonstration is made to and approved by TDEC that certain post-closure activities are no longer required to protect human health and the environment.

\subsection{Security Requirements \\ Ref: $\quad 1200-1-11-.06(7)$ \\ 40 CFR 264.117(b)}

Security at the KHQ will be maintained for 30 years after closure. Access to the facility is limited to authorized personnel and is controlled by a chain-link fence with a lockable gate at the single access road. Signs have been posted around KHQ prohibiting unauthorized entry.

\subsection{Property Use Requirements}

Ref: $\quad 1200-1-11-.06(7)$

40 CFR 264.117(c)

Land use at and near KHQ shall be restricted to ensure the unit is maintained and to prevent the disturbance of the monitoring system and security fence. 


\subsection{Submittal of Post-Closure Plan}

Ref: $\quad 1200-1-11-.06(7)$

40 CFR 264.118(a) and 270.14(b)(13)

This Post-Closure Plan is being submitted as a part of the KHQ Post-Closure Permit Application.

\subsection{Availability of the Post-Closure Plan}

Ref: $\quad 1200-1-11-.06(7)$

40 CFR 264.118(b)(3)

The name, address, and phone number of the contact for the KHQ during the post-closure care period are listed below. A copy of the updated post-closure plan for the KHQ will be maintained in this office during the post-closure care period.

U.S. Department of Energy, Environmental Restoration Division

P.O. Box 2001

Oak Ridge, Tennessee 37831-8012

(615) 576-0715

\subsection{Content of the Post-Closure Plan \\ Ref: $\quad 1200-1-11-.06(7)$ \\ 40 CFR 264.118(b)}

The activities discussed in this section are intended to meet the content requirements found in TN Rule 1200-1-11-.06(7) and 40 CFR 264.118 as incorporated by reference by the Tennessee Hazardous Waste Management Regulations.

\subsection{Monitoring Activities}

Ref: $\quad 1200-1-11-.06(7)$

40 CFR 264.118(b)(1)

Groundwater monitoring activities to be performed upon issuance of a Post-Closure Permit for the KHQ are presented in Section B.5 of the "Post-Closure Permit Application for the Kerr Hollow Quarry at the Y-12 Plant." 


\subsection{Maintenance Activities}

Ref: $\quad 1200-1-11-.05(7)$

40 CFR 264.118(b)(2)

Closure activities at the $\mathrm{KHQ}$, as required by the approved closure plan, included the removal of visible items and materials on the bottom of the quarry in order to remove potentially untreated hazardous wastes. A standard cap was impractical due to the quantity of water flowing into the quarry from the surrounding aquifer and was not included in the approved closure activities. Therefore, maintenance at the KHQ will be limited to inspection of the security fence, gate, signs, benchmarks, and groundwater monitoring system. Inspections will be conducted on a quarterly basis for all components of the KHQ. The monitoring wells associated with the KHQ will be subject to a comprehensive inspection on an annual basis. Table 1 identifies the items to be inspected and corrective actions to be taken.

All detection monitoring wells will be inspected annually by sounding the well. Rehabilitation or redevelopment will be conducted on those wells in which the sounding results indicate a well depth change of 20 percent or greater of the total screen length or of the open interval. In addition, as a part of the inspection program, all monitoring wells will be inspected to check for proper identification, damage, and to ensure that the protective caps are locked.

Following each inspection, the inspector will complete a Post-Closure Inspection Checklist for the site. Examples of inspection checklists are included in this Post-Closure Plan as Figures 1 and 2. These checklists will be maintained as required during the post-closure care period at the KHQ. 
Table 1. Quarterly Inspection and Maintenance Plan for the Kerr Hollow Quarry

\begin{tabular}{ll}
\multicolumn{1}{c}{ Inspection } & \multicolumn{1}{c}{ Corrective Action } \\
\hline $\begin{array}{l}\text { Monitoring Wells } \\
\text { - Check for damage, make sure protective } \\
\text { caps are locked. ID tags or numbers in } \\
\text { place and legible. }\end{array}$ & $\begin{array}{l}\text { Notify GWPPM if repairs cannot be made, P\&A } \\
\text { damaged well and construct replacement well with } \\
\text { TDEC concurrence. Lock any unlocked caps. }\end{array}$ \\
$\begin{array}{ll}\text { Unauthorized Deliveries } \\
\text { - Check for any extraneous materials at site. }\end{array}$ & Ensure all unauthorized materials are removed. \\
Signs & \\
- Check for any damaged or missing signs. & $\begin{array}{l}\text { Ensure damaged or missing signs are repaired or } \\
\text { replaced. }\end{array}$ \\
Benchmarks & \\
- Check for damage. & Ensure damaged benchmarks are replaced. \\
\hline
\end{tabular}

Source: Energy Systems, Inc., Y-12 Plant, Oak Ridge, Tennessee 
Inspector's Name and Title:

Date of Inspection:

Time of Inspection:

Supervisor's Signature:

\begin{tabular}{|c|c|c|c|c|c|}
\hline Inspection & $\begin{array}{l}\text { Type of } \\
\text { Problem }\end{array}$ & $\begin{array}{c}\text { Acceptable } \\
\text { Unacceptable }\end{array}$ & Observations & Corrective Action & $\begin{array}{c}\text { Date Action } \\
\text { Requested }\end{array}$ \\
\hline $\begin{array}{l}\text { Monitoring } \\
\text { Wells }\end{array}$ & $\begin{array}{l}\text { Signs of } \\
\text { damage, } \\
\text { protective caps } \\
\text { unlocked }\end{array}$ & & & $\begin{array}{l}\text { Notify GWPPM. If } \\
\text { repairs cannot be } \\
\text { made, P\&A } \\
\text { damaged wells and } \\
\text { replace with TDEC } \\
\text { concurrence. Lock } \\
\text { any unlocked caps. }\end{array}$ & \\
\hline $\begin{array}{l}\text { Unauthorized } \\
\text { Deliveries }\end{array}$ & $\begin{array}{l}\text { Extraneous } \\
\text { materials at site }\end{array}$ & & & $\begin{array}{l}\text { Ensure all } \\
\text { unauthorized } \\
\text { materials are } \\
\text { removed. }\end{array}$ & \\
\hline Signs & $\begin{array}{l}\text { Damaged or } \\
\text { missing signs }\end{array}$ & & & $\begin{array}{l}\text { Ensure damaged or } \\
\text { missing signs are } \\
\text { repaired or } \\
\text { replaced. }\end{array}$ & \\
\hline Benchmarks & $\begin{array}{l}\text { Check for } \\
\text { damage }\end{array}$ & & & $\begin{array}{l}\text { Ensure damaged } \\
\text { benchmarks are } \\
\text { replaced. }\end{array}$ & \\
\hline
\end{tabular}

- Photograph representing deteriorated conditions required before repairs.

Source: Energy Systems, Inc., Y-12 Plant, Oak Ridge, Tennessee

Figure 1. Example of a Post-Closure Inspection Checklist. 


\section{Y-12 PLANT GROUNDWATER PROTECTION PROGRAM \\ WELL INSPECTION CHECKLIST \\ INSPECTION NO:}

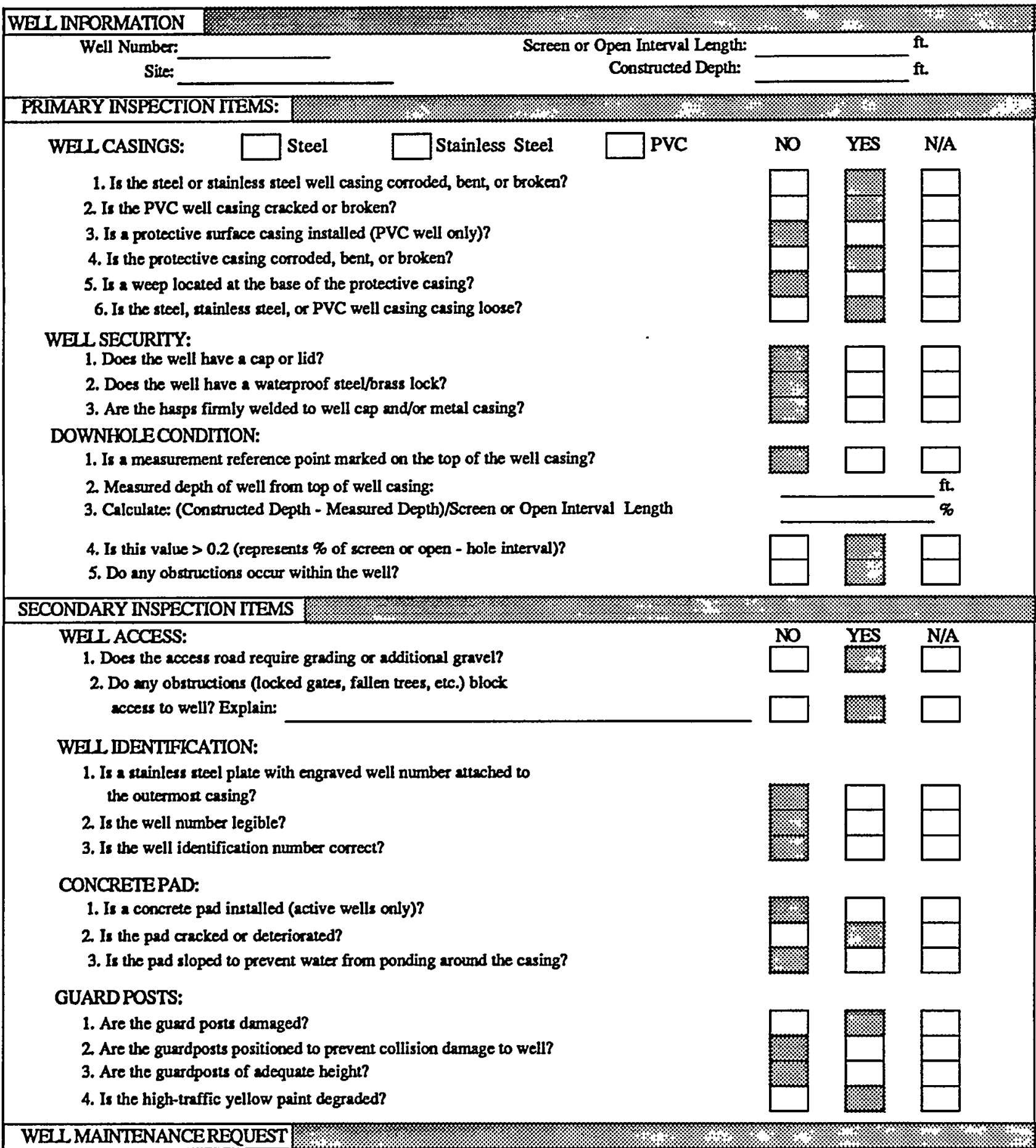

Complete only if any of the above shaded yes/no boxes are checked.

Primary Item(s)

Maintenance Request No.

COMMENTS $\mathrm{Z}$ :

Figure 2. Example of a well inspection checklist.

$$
\text { A.2-6 }
$$


Site maintenance will be conducted to repair any damage or correct site conditions at KHQ. If monitoring wells are damaged such that samples cannot be obtained, the wells will be plugged and replaced upon receiving written concurrence from the Commissioner. The damaged well will be plugged and abandoned in accordance with the current version of the procedure found in Appendix C.1 of the Post-Closure Permit Application for the KHQ.

\subsection{Post-Closure Contact}

Ref: $\quad 1200-1-11-.06(7)$

40 CFR 164.118(b)(3)

The post-closure contact is identified in Section 3.0 of this plan.

\subsection{Amendment of the Post-Closure Plan}

Ref: $\quad 1200-1-11-.06(7)$

40 CFR 264.118(d)

In the event that operating plans or facility design changes affect post-closure activities at the KHQ, an amended post-closure plan will be submitted to the Commissioner. The post-closure plan will be amended at least 60 days prior to the proposed change in facility design or operation, or not later than 60 days after an unexpected event has occurred which affects the post-closure plan.

\subsection{Post-Closure Notices}

Ref: $\quad 1200-1-11-.06(7)$

40 CFR 264.119

Closure of the KHQ has been completed and notices as discussed in Section A of this permit application have been submitted. A plat map and waste description have been provided to the Anderson County, Tennessee Zoning Authority and TDEC. No record of deed has been made since the U.S. Department of Energy (DOE) Oak Ridge Reservation property does not have a deed. If, in the future, any Y-12 Plant property on which hazardous waste has been managed is to be released from federal government ownership for private development, information regarding these activities will be placed in a legal document which would be examined during a title search. 


\subsection{Certification of Completion of Post-Closure Care}

Ref: $\quad 1200-1-11-.06(7)$

40 CFR 264.120

Upon completion of the post-closure care activities at the KHQ, DOE will submit a certification to the Commissioner. This certification will be submitted no later than 60 days after completion of the established post-closure care period. The certification will be made by the owner/operator and an independent professional registered engineer that post-closure activities were conducted in accordance with the approved post-closure plan and permit.

\subsection{Post-Closure Cost Estimate \\ Ref: $\quad 1200-1-11-.06(8)$ \\ 40 CFR 264.140(c)}

In accordance with the above references, federal facilities are exempt from providing postclosure care cost estimates.

\subsection{Adjustments to Post-Closure Care Cost Estimates}

Ref:' $\quad 1200-1-11-.06(8)$

40 CFR 264.140(c)

In accordance with the above references, federal facilities are exempt from the requirements concerning the submittal of cost estimates.

\subsection{Revisions to Post-Closure Care Cost Estimates}

Ref: $\quad 1200-1-11-.06(8)$

40 CFR 264.140(c)

In accordance with the above references, federal facilities are exempt from the requirements concerning the submittal of cost estimates.

\subsection{Financial Assurance for Post-Closure Care}

Ref: $\quad 1200-1-11-.06(8)$

40 CFR 264.140(c)

In accordance with the above references, federal facilities are exempt from the requirements pertaining to financial assurance. 


\section{A.3 CLOSURE CERTIFICATION LETTER}




\title{
MAIL
}

\author{
STATE OF TENNESSEE \\ DEPARTMENT OF ENVIRONMENT AND CONSERVATION \\ Division of Solid Waste Management \\ Fifth Floor, L \& C Tower \\ 401 Church Street \\ Nashville, Tennessee 37243-1535
}

February 22, 1995

Certified Mail

No. P 198160759

Return Receipt Requested

Mr. Robert C. Sleeman

Environmental Restoration Division

U.S. Department of Energy

Oak Ridge Operations Office

Post Office Box 2001

Oak Ridge, Tennessee 37831-8541

\section{Re: Closure Certification \\ Kerr Hollow Quarry \\ Y-12 Plant \\ EPA I.D. No.: TN3 890090001}

Dear Mr. Sleeman:

This letter is to inform you that the Tennessee Department of Environment and 'Conservation's Division of Solid Waste Management has completed its review of the Owner/Operator/Co-Operator and Independent Registered Professional Engineer Closure Certifications submitted for the above referenced site.

The Department concurs that closure has been performed in accordance with the approved closure. plan and in compliance with the closure performance standards of 40 CFR 265.111 as incorporated by reference at Rule 1200-1-11$.05(7)(2)$.

Please recognize that closure of these units means that you are no longer authorized to operate these units under interim status. If, in the future, you wish to conduct operations that require a permit, you must first apply for and obtain that permit from this Department. 
Mr. Robert C. Sleeman

February 22, 1995

Page 2

If you have any questions, please contact Mr. Edward Cox of my staff at (615) 532-0827.

Sincerely,

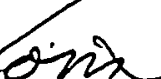

Tom Tiesler, Director

Division of Solid Waste Management

$\mathrm{JTT} / \mathrm{mec}$

cc: Ms. Jacqueline Okoreeh-Baah, Chief, Combustion Unit Mr. Wayne Gregory, Chief, HW Permitting Unit

Mr. Bill Krispin, Chief, RCRA Land Unit

Mr. Earl Leming, Director, DOE Oversight Division

Mr. G. Alan Farmer, EPA, Region IV

Ms. Sherry Lankford, U. S. DOE

Mr. Tom Perry, Martin Marietta Energy Systems, Inc.

Mr. Mark Burris, MMES 


\section{APPENDIX B}

\section{WELL CONSTRUCTION DIAGRAMS}




\section{WELL \\ CONSTRUCTION \\ SUMMARY}

WELL NO. GW-142

LOCATION Kerr Hollow

Quarry

DRILLING COMPANY: GEOTEK

DRILLERS: R.M. Collins/Randy Collins

DATE COMPLETED:

3 Oct 1985

TOTAL DEPTH: $295^{\circ}$

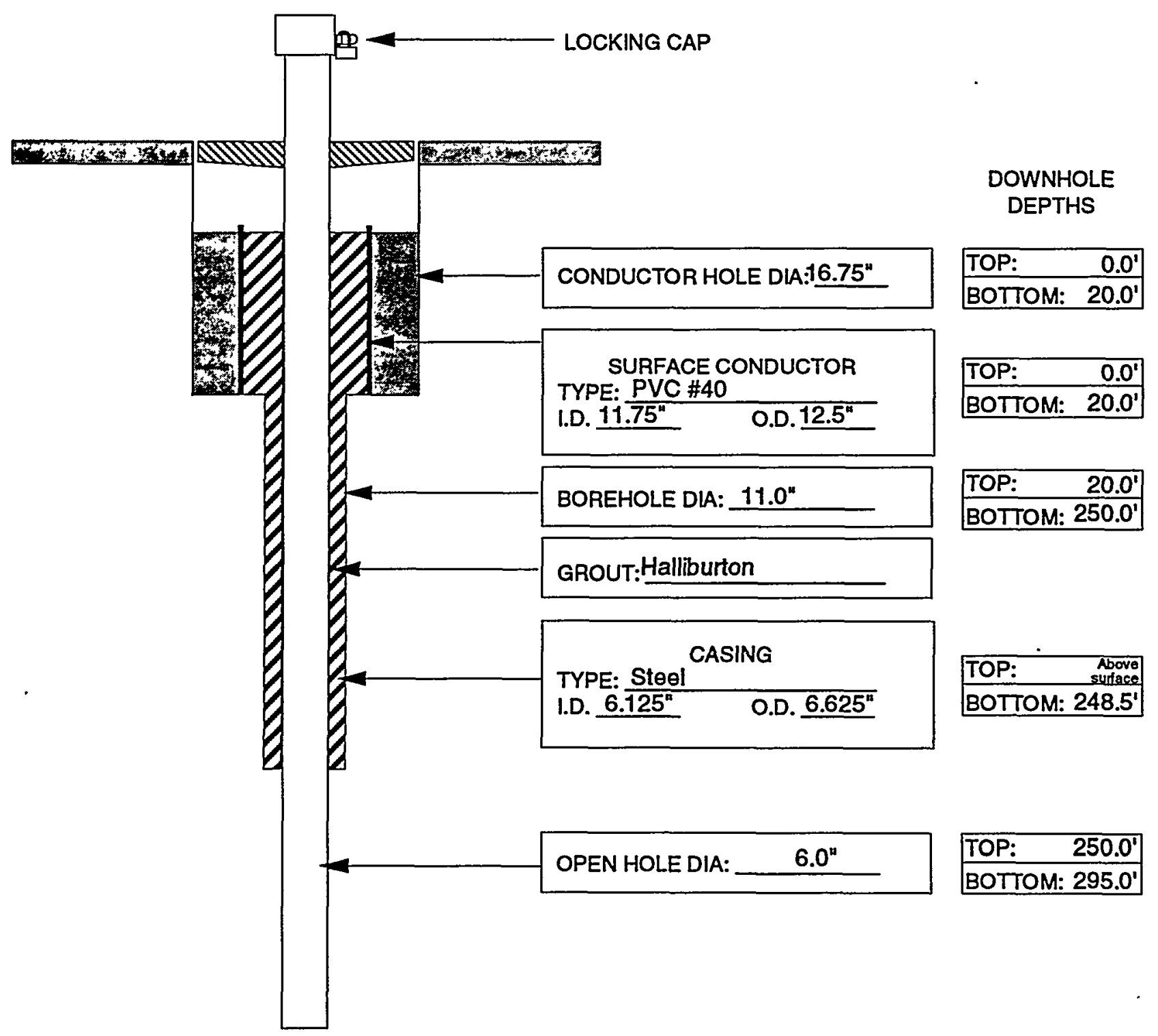

NOT TO SCALE 


\section{WELL \\ CONSTRUCTION \\ SUMMARY}

WELL NO. GW-143

LOCATION Kerr Hollow Quarry

DRILLING COMPANY: GEOTEK

DRILLERS: R.M. Collins/Randy Collins

DATE COMPLETED:

24 Oct 1985

TOTAL DEPTH: $253^{\prime}$

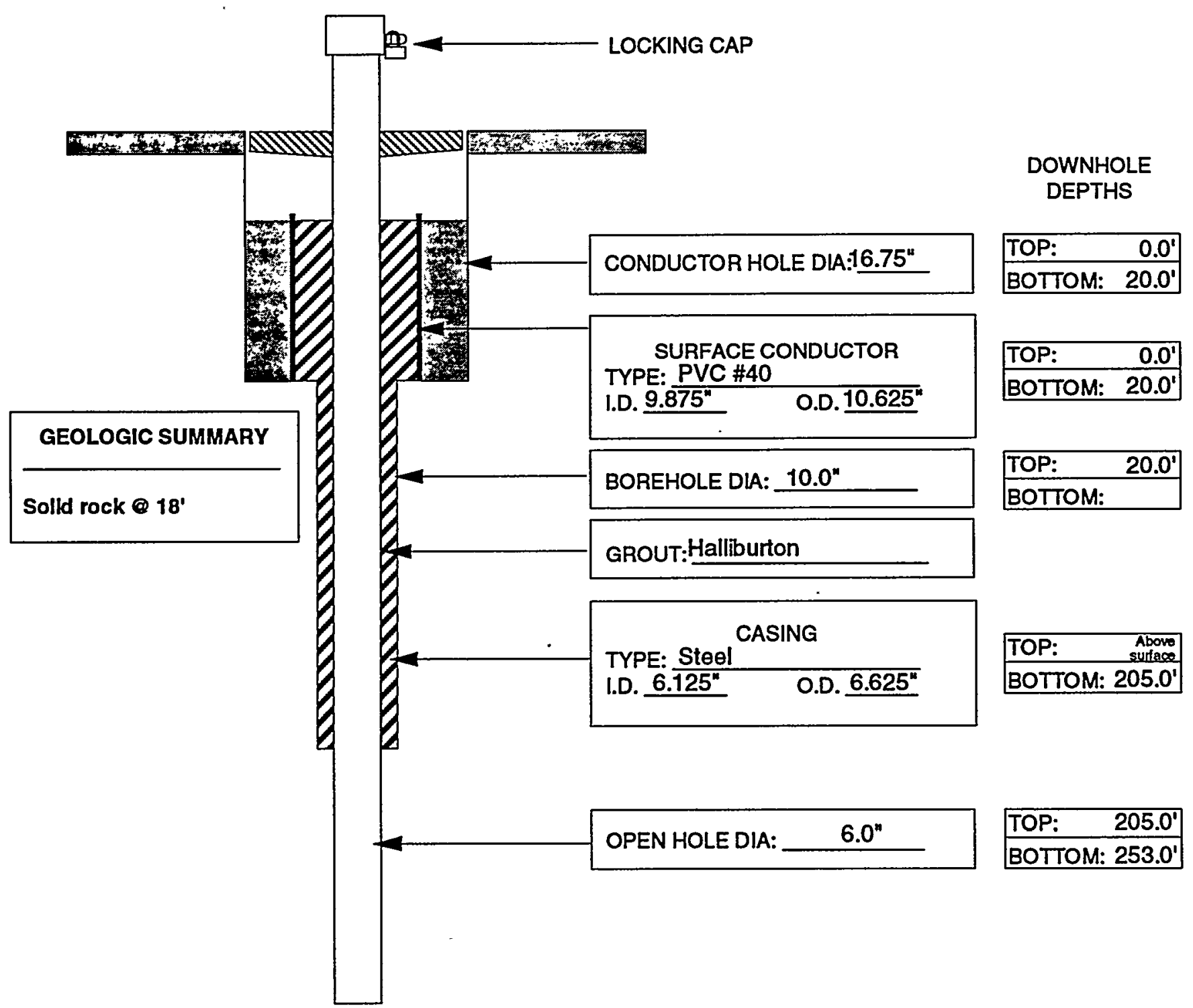

NOT TO SCALE 


\begin{tabular}{|l|l|}
\hline DRILLERS: R.M. Collins/Randy Collins & $\begin{array}{l}\text { WELL NO. GW-144 } \\
\text { LOCATION Kerr Hollow }\end{array}$ \\
\hline Quarry \\
\hline DRILLING COMPANY: GEOTEK
\end{tabular}

NOT TO SCALE 


\section{WELL \\ CONSTRUCTION \\ SUMMARY}

WELL NO. GW-145

LOCATION Kerr Hollow Quarry

DRILLING COMPANY: GEOTEK

DRILLERS: R.M. Collins/Randy Collins

DATE COMPLETED: 14 Oct 1985

TOTAL DEPTH: $110^{\prime}$

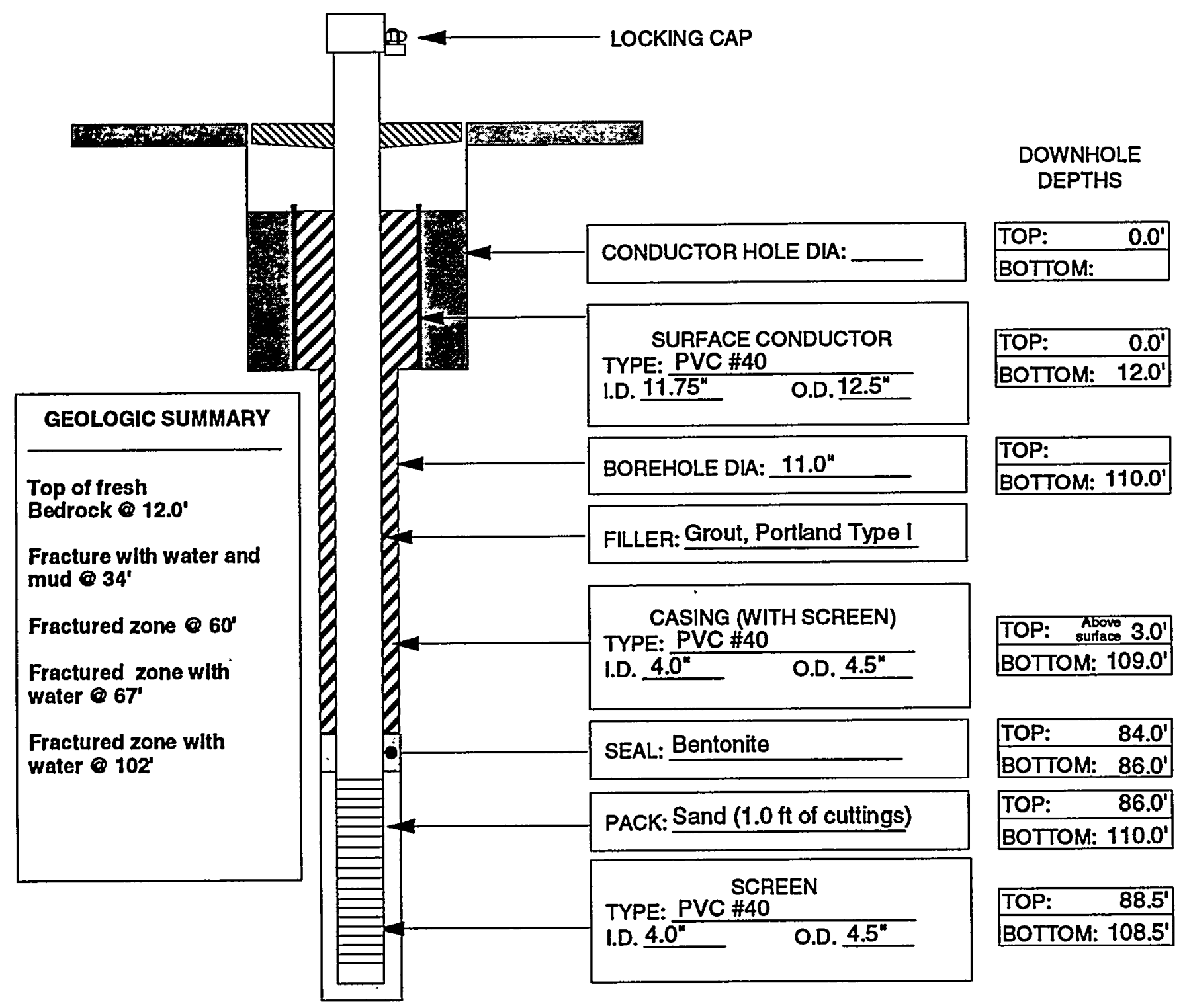

NOT TO SCALE 


\begin{tabular}{|l|l|}
\hline DRILLNG COMPANY: GEOTEK & $\begin{array}{l}\text { WELL NO. GW-231 } \\
\text { LOCATION Kerr Hollow }\end{array}$ \\
\hline QULarry \\
\hline NOTE: Surface conductor temporary-not grouted.
\end{tabular}

NOT TO SCALE 


\begin{abstract}
APPENDIX C
APPLICABLE STANDARD PRACTICE PROCEDURES FOR THE GROUNDWATER PROTECTION PROGRAM
\end{abstract}

C.1 WELL PLUGGING AND ABANDONMENT

C.2 WELL INSPECTION PROCEDURE

C.3 WELL DEPTH MEASUREMENT PROCEDURE 


\section{C.1 WELL PLUGGING AND ABANDONMENT}


Y-12 PLANT GROUNDWATER PROTECTION PROGRAM |G-003

Rev. 1,

TITLE: Well Plugging and Abandonment Procedure

October 1994

Page 2 of 18

CONTENTS

Page

1.0 PURPOSE . . . . . . . . . . . . . . . . . . . . . . . . . 4

2.0 APPLICABIIITY . . . . . . . . . . . . . . . . . . . . . . 4

3.0 DEFINITIONS . . . . . . . . . . . . . . . . . . . . . . . . . 4

4.0 REFERENCES . . . . . . . . . . . . . . . . . . . . . . . 5

5.0 PRECAUTIONS AND LIMITATIONS . . . . . . . . . . . . . . . 6

5.1 Cavities and Fractures . . . . . . . . . . . . 6

5.2 Method Selection . . . . . . . . . . . . . . 6

5.3 Safety . . . . . . . . . . . . . . . . . . . . . . 7

5.4 Cement Slurry Weights and Curing Schedules . . . . 7

5.5 Casing Extraction . . . . . . . . . . . . . . . . 7

5.6 Well Construction Information . . . . . . . . . . . . 7

6.0 EQUIPMENT, TOOLS AND SUPPLIES . . . . . . . . . . . . . . 8

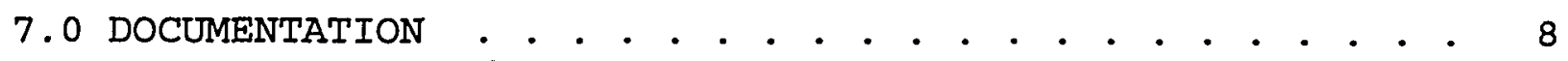

8.0 PLUGGING AND ABANDONMENT . . . . . . . . . . . . . . . . . . . . 9

8.1 Site Preparation . . . . . . . . . . . . . . . 9

8.2 Equipment Decontamination . . . . . . . . . . . . 9

8.3 Method A . . . . . . . . . . . . . . . 10

8.3.1 Remove the Well Casing. . . . . . . . 10

8.3.2 Condition the Borehole. . . . . . . . . . . 10

8.3.3 Set the Plug.............. . . 10

8.3.4 Remove the Surface/Conductor Casing . . . 11

8.3.5 Verify Plug Depth ............ . . 11

8.3.6 Cap the Plug... . . . . . . . . . . . . . 11

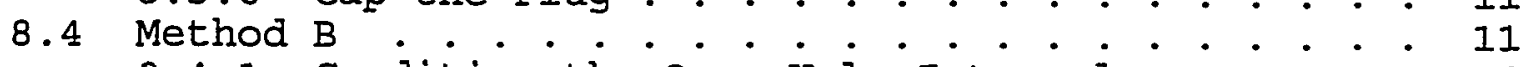

8.4.1 Condition the Open-Hole Interval . . . . . 12

8.4.2 Set the Lower Plug . . . . . . . . . . . . 12

8.4.3 Remove the Well Casing. . . . . . . . . . 12

8.4.4 Condition the Borehole... . . . . . . . . 12

8.4.5 Set the Upper Plug . . . . . . . . . . . . . 12

8.4.6 Remove the Surface/Conductor Casing . . . . 13

8.4.7 Verify Plug Depth ............. . 13

8.4.8 Cap the Plug .. . . . . . . . . . . . . 13

8.5 Method C . . . . . . . . . . . . . . . . . . . . . 13

8.5.1 Remove the Well Casing. . . . . . . . . . 14

8.5.2 Condition the Borehole.......... . . 14

8.5.3 Set the Plug................ 14 
Y-12 PLANT GROUNDWATER PROTECTION PROGRAM

TITLE: Well Plugging and Abandonment Procedure
G-003

Rev. 1,

October 1994

Page 3 of 18

\section{CONTENTS (Cont'd)}

Page

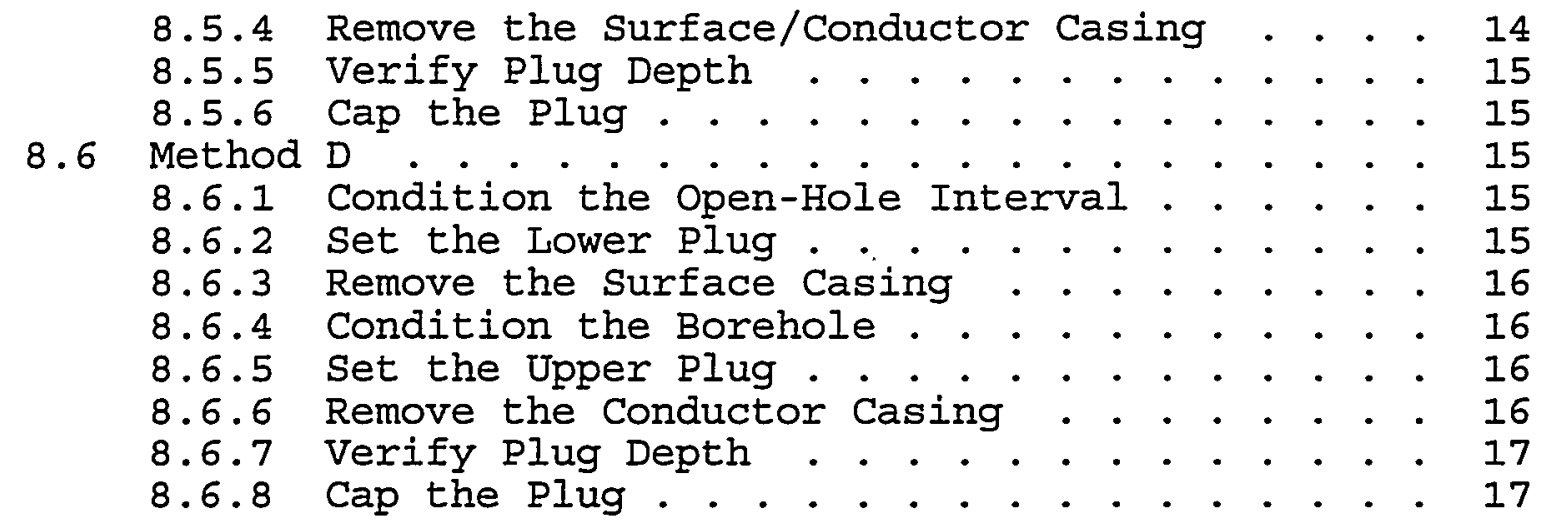

9.0 ACCEPTANCE CRITERIA . . . . . . . . . . . . . 17

10.0 POST PERFORMANCE WORK ACTIVITIES . . . . . . . . . . 17

11.0 RECORDS . . . . . . . . . . . . . . . . 18 


\section{Y-I2 PLANT GROUNDWATER PROTECTION PROGRAM}

TITLE: Well Plugging and Abandonment Procedure

G-003

Rev. 1, October 1994 Page 4 of 18

\subsection{PURPOSE}

This procedure contains guidelines and methods for well plugging and abandonment (P\&A) at the Oak Ridge Y-12 Plant. Wells of similar construction materials and design are classified into one of four groups, and a specific P\&A method is defined for each group. The methods are designed to remove all well materials, seal the borehole to prevent fluid migration into or between water-bearing zones, and to minimize the amount of waste-materials generated during $P \& A$ operations.

\subsection{APPIICABILITY}

This procedure applies to all wells at the Y-12 Plant which are designated for P\&A by the Y-12 Plant Groundwater Protection Program Manager (GWPPM) or authorized designee.

Annular seal - material (grout or cement) which prevents fluid migration through the space between the well casing and borehole wall or outer casing.

Conductor Casing - an initial casing, typically steel or PVC, installed in the unconsolidated zone to support the borehole and provide drilling rig stability. This casing may be removed during completion of the well or grouted in place.

Containment system - excavated pit, drums, tanks or other containers used to collect and contain drill cuttings and fluids generated during $P \& A$.

Diverter Assembly - apparatus used to direct drill cuttings and fluids to the containment system.

Groundwater Protection Program (GWPP) - program established to monitor groundwater quality and hydrologic conditions at the $\mathrm{Y}-12$ Plant.

GWPP Managex (GWPPM) - person responsible for day-to-day management of the $\mathrm{Y}-12$ Plant GWPP. 


\section{Y-12 PLANT GROUNDWATER PROTECTION PROGRAM}

G-003

Rev. 1,

TITLE: Well Plugging and Abandonment Procedure

October 1994

Page 5 of 18

On-Site Geologist - a professional geologist, registered in the state of Tennessee, responsible for field supervision of P\&A operations.

Open-Hole Interval - the bottom section of a well drilled in competent rock unsupported by well screen or casing.

Primary Inspection Item - those monitoring well components that are critical to the collection of representative groundwater samples and/or hydrologic data from the well. Includes the well casing and annular grout seal, well security, and the downhole condition of the well.

Protective Well Casing - a section of large diameter steel or polyvinyl chloride (PVC) pipe that is emplaced over the upper end of a smaller diameter monitoring well casing to provide structural protection to the well and restrict unauthorized access to the well.

Surface Casing - steel or PVC piping set from the ground surface into the top of bedrock to support the unconsolidated section of the borehole. The surface casing in a core hole extends into bedrock to the top of the open-hole interval.

Tremie Method - a method for placing cement in the borehole. Cement is pumped through a small diameter pipe (usually 2 -in or less) extending to at least 1 -ft above the bottom of the borehole or the top of a previously placed cement plug. The pipe is raised as the cement is emplaced. Use of this method reduces the potential for the cement to bridge and ensures placement of cement along the entire length of the borehole.

Washover Pipe - a drill pipe which fits around the well casing and is used to drill out the annular grout seal.

Well Casing - steel, stainless steel or PVC piping which provides unobstructed access to the monitored interval.

\subsection{REFERENCES}

4.1 Aller, Iinda, Truman W. Bennett, Gene Hackett, Rebecca J. Petty, Jay H. Lehr, Helen Sedoris, and David M. Nielsen. Handbook of Suggested Practices for the Design and Installation of Groundwater Monitoring Wells, National Water Well Association, Dublin, Ohio, $398 \mathrm{p}$. 


\section{Y-12 PLANT GROUNDWATER PROTECTION PROGRAM}

TITLE: Well Plugging and Abandonment Procedure

G-003

Rev. 1, October 1994

Page 6 of 18

4.2 Driscoll, Fletcher G. 1986. Groundwater and WelIs, Johnson Division, St. Paul, Minnesota, 1089 p.

4.3 Martin Marietta Energy Systems, Inc. 1987. Environmental Surveillance Procedures Quality Control Program, $\mathrm{ESH} / \mathrm{SUB} / 87 / 21706 / 1$.

4.4 Martin Marietta Energy Systems, Inc. 1987. Plugging and Abandonment Procedures for the Oak Ridge Y-12 Plant, Y/TS-531.

4.5 Martin Marietta Energy Systems, Inc. 1994. Monitoring Well Inspection and Maintenance Plan, Y-12 Plant, Oak Ridge, Tennessee (Revised), Y/TS-1215.

4.6 Halliburton Services, Inc., 1981, Halliburton Cementing Tables, Iittle's, Duncan, Oklahoma.

4.7 Jones, S.B., B. K. Thompson, and S. M. Field, 1994, Updated Subsurface Data Base for Bear Creek Valley, Chestnut Ridge, and Parts of Bethel Valley on the U.S. Department of Energy Oak Ridge Reservation, $Y / T S-881 / R 2$.

\subsection{PRECAUTIONS AND LIMITATIONS}

\subsection{Cavities and Fractures}

Cavities, fractures, joints, bedding planes, or other voids may be encountered during removal of the well casing and reaming of the borehole, resulting in a greater volume of cement to plug the borehole than calculated from the borehole depth and diameter. Additionally, lost-circulation additives may be required to minimize fluid loss during $P \& A$ operations.

\subsection{Method Selection}

Because of differences in well construction and conditions that may occur during drilling operations, not all of the monitoring wells at the Y-12 Plant can be classified into one of the four categories of well construction. A flexible policy is necessary to determine the most appropriate $P \& A$ method for each well and to allow some deviation from the specified method as conditions warrant. 
Y-12 PLANT GROUNDWATER PROTECTION PROGRAM

TITLE: Well Plugging and Abandonment Procedure

G-003

Rev. 1,

October 1994

Page 7 of 18

\subsection{Safety}

Established safety standards and requirements of Martin Marietta Energy Systems, Inc., the Department of Energy (DOE), and the Occupational safety and Health Administration (OSHA) will apply to all P\&A field operations as outlined in Health and Safety Plan for Well Installation and Plugging and Abandonment Activities, Y12 Plant, Oak Ridge, Tennessee (Y/SUB/92-99928C(Y11)/1, July 1992 .

\subsection{Cement slurry Weights and Curing Schedules}

The length of the column of cement emplaced at one time should not exceed 300 ft so that the weight of the cement is less than the fracture pressure of the monitored formation. This will also minimize infiltration of cement into the formation. Cement cure times shall be determined by the on-site geologist. Cure times will be specified depending on temperature and required compressive strength using guidelines established in Reference 4.6 .

\subsection{Casing Extraction}

Certain monitoring wells have been constructed using substandard annular seals or no annular seal. Casings in these wells may be extracted without use of overwashing techniques. In cases where the construction of the well allows, an attempt to extract the well casing may be made using the drilling rig head and appropriate lifting device, such as lifting bell, clevis, wire rope, or chain. Casing extraction jacks may alternately be used. Drilling rig leveling jacks or winches shall not be used to attempt to extract casings prior to overwashing. The decision to attempt to extract a well casing shall be made by the on-site geologist, in conjunction with the GWPPM or authorized designee, and documented as specified in section 7.0.

\subsection{Well Construction Information}

Data contained in Reference 4.7 is compiled from best available records. However, erroneous and missing well construction data exists, particularly for older wells that pre-date the GW Series. Where well construction data is unavailable, best technical judgment as to casing 
Y-12 PLANT GROUNDWATER PROTECTION PROGRAM

TITLE: Well Plugging and Abandonment Procedure
G-003

Rev. 1,

October 1994

Page 8 of 18

set points or well depths will be employed by the on-site geologist in consultation with the GWPPM or authorized designee. As a result, deviations from the standard P\&A likely will be required. All deviations shall be recorded as outlined in section 9.0.

\subsection{EQUIPMENT, TOOLS AND SUPPLIES}

6.1 Fully Equipped Drilling Rig: includes but is not limited to drill bits, washover pipe, diverter assembly, etc.

6.2 Grouting Supplies and Equipment: cement, additives, potable water, mixer, pump, and tremie pipe.

6.3 Containment System (as required): excavated containment pit, drums, tanks, and/or other containers.

6.4 Safety Equipment (as required): includes but is not limited to safety shoes, tyvek coveralls, protective eyewear, hard hat, and rubber gloves.

6.5 Decontamination Equipment (as required) : includes but is not limited to steam cleaner, potable water, and mild detergent.

\subsection{DOCUMENTATION}

7.1 A Well Plugging and Abandonment Request Form is used to initiate P\&A activities, and is completed by the $\mathrm{Y}-12$ Plant GWPPM or authorized designee if: (1) a well impedes site operations, construction or closure, (2) inspection of a well has indicated significant damage to or deterioration of a Primary Inspection Item, or (3) the Y-12 Plant GWPPM or authorized designee determines that P\&A of a well is warranted for other reasons. The completed form is transmitted to the on-site geologist when P\&A operations are scheduled.

7.2 Before P\&A operations begin, a Well Plugging and Abandonment Waste Management Plan is completed by the Y12 Plant GWPPM or authorized designee. The plan includes: (1) the estimated volume of cuttings and fluids that will be generated during $P \& A$, (2) the types and concentrations of contaminants (if any) known to be present in the well, (3) the appropriate waste containment method required during $P \& A$ operations (i.e., 
discharge to ground surface or containment system), (4) an estimate of the number and types of samples (e.g., cuttings) to be collected during $P \& A$ and the required analyses of the samples prior to disposal or treatment, and (5) the proposed disposition or treatment of any containerized materials.

7.3 Well Plugging and Abandonment Diagrams are completed by the Y-12 Plant GWPP Manager or authorized designee, and the on-site geologist. Before P\&A operations begin, the Y-12 Plant GWPP Manager or authorized designee completes the following sections of the diagram: (1) the well Iocation (site), (2) the drilling subcontractor, (3) the rationale for $P \& A$ of the well, (4) the $P \& A$ method (including any proposed deviations from the specified method), and (5) applicable well construction details (e.g., borehole diameter). During P\&A operations, the on-site geologist completes the diagram with specific P\&A details for the well (e.g., depth to the top of the cement plug).

7.4 A Well Plugging and Abandonment Activity/Progress Report is completed by the on-site geologist and includes descriptions of the daily activities performed during P\&A operations.

\subsection{PLUGGING AND ABANDONMENT}

\subsection{Site Preparation}

8.1.1 Confirm well identification and site access, and mobilize drilling and grouting equipment to the well site.

8.1.2 Remove the surface features from the well (1ock, well cap, guard posts, surficial concrete pad, protective well casing or manhole cover) as applicable.

8.1.3 If specified in the Well Plugging and Abandonment Waste Management Plan, set up the diverter assembly and the containment system.

\subsection{Equipment Decontamination}

If specified in the Well Plugging and Abandonment Waste Management Plan, decontaminate the drilling and associated equipment (e.g., drill bits, drill rods, 
Y-12 PLANT GROUNDWATER PROTECTION PROGRAM

TITLE: Well Plugging and Abandonment Procedure
G-003

Rev. 1, October 1994 Page 10 of 18

tremie pipe) when P\&A operations at each well have been completed.

\subsection{Method A}

Method A is for wells constructed of 7 inch (in) outside diameter (OD) or smaller steel or stainless steel well casing, and typically completed with 5 to 20-ft well screens and sand filter packs. Wells completed in bedrock may also have 8- to 12-in-OD steel or PVC surface casing extending from ground surface to the top of bedrock. Some wells may also be completed with a conductor casing.

\subsubsection{Remove the Well Casing}

Drill out the annular grout seal using a washover pipe advanced to the bottom of the borehole. Retrieve the washover pipe and remove the well casing. Staged removal of the casing string may be necessary if it cannot be removed in one operation. In the event that a borehole is deviated, stainless steel casing may be drilled out with a tri-cone drill bit. If this approach is feasible, the bit size must be at least 0.25 in larger than the original borehole diameter to ensure that the casing and annular grout seal are completely removed.

\subsubsection{Condition the Borehole}

If the well was completed in bedrock, ream the borehole with a tri-cone drill bit that is at least 0.25 -in larger in diameter than the original borehole. This will expose fresh bedrock and help ensure an effective bond between the cement plug and the borehole wall.

\subsubsection{Set the Plug}

Tremie API Class A neat cement, mixed with potable water to a slurry density of 12 to 15 pounds per gallon (1bs/gal), from the bottom of the borehole. If no surface/conductor casing is present, tremie the cement to within 4 ft of ground surface. If a surface/conductor casing is present, tremie the cement to within 4 ft of the bottom of the casing. 
Y-12 PLANT GROUNDWATER PROTECTION PROGRAM

TITLE: Well Plugging and Abandonment Procedure
G-003

Rev. 1, October 1994

Page 11 of 18

\subsubsection{Remove the Surface/Conductor Casing}

If the well was completed with surface/conductor casing, drill out the annular grout seal using a washover pipe advanced to the bottom of the casing. Retrieve the washover pipe and remove the casing. Using a tri-cone drill bit at least 0.25 -in larger in diameter than that of the original borehole, ream the borehole to the top of the existing cement plug. Tremie cement, mixed to a density of 12 to $15 \mathrm{lbs} / \mathrm{gal}$, from the top of the existing plug to within 4 ft of the ground surface (or bottom of conductor casing, if removing a surface casing).

It may be possible to remove PVC casing by drilling it out with a tri-cone drill bit. If this approach is feasible, the bit size must be at least 0.25 -in larger than the original borehole diameter to ensure that the casing and annular grout seal are completely removed.

\subsubsection{Verify Plug Depth}

Measure the depth to the top of the cement plug to verify that it is within 4 ft of the ground surface. If not, add more cement until the specified depth is reached.

\subsubsection{Cap the Plug}

Fill the remainder of the borehole to ground surface with compacted non-contaminated soil.

\subsection{Method B}

Method $B$ is for wells constructed of 7-in-OD or smaller steel or PVC well casing completed with open-hole intervals in competent bedrock. The well casing typically extends from the ground surface to the top of the open-hole interval, which typically extends from 5 to $100 \mathrm{ft}$ below the bottom of the well casing. The wells may also be completed with 8- to 12-in-OD steel or PVC surface casing extending from ground surface to the top of bedrock. Some wells may also be completed with a conductor casing. 
Y-12 PLANT GROUNDWATER PROTECTION PROGRAM

TITLE: Well Plugging and Abandonment Procedure
G-003

Rev. 1, October 1994

Page 12 of 18

\subsubsection{Condition the Open-Hole Interval}

Lower a drill string and tri-cone drill bit into the well. Circulate air and potable water containing additives such as QUIK-GEI or QUICKMUD to remove any old cuttings and debris that may have accumulated at the bottom of the well. After the initial circulation, drill approximately $1 \mathrm{ft}$ below the bottom of the well to expose fresh rock, and repeat the well circulation procedure to remove the cuttings.

\subsubsection{Set the Lower Plug}

Tremie API Class A neat cement, mixed with potable water to a slurry density of 12 to 15 lbs/gal, from the extended bottom of the openhole interval to within $4 \mathrm{ft}$ of the bottom of the well casing.

\subsubsection{Remove the Well Casing}

Drill out the annular grout seal surrounding the well casing using a washover pipe advanced to the top of the open-hole interval. Retrieve the washover pipe and remove the casing. Staged removal of the casing string may be necessary if it cannot be removed in one operation.

It may be possible to remove PVC well casing by drilling it out with a tri-cone drill bit. If this approach is feasible, the bit size must be at least 0.25 -in larger than the original borehole diameter to ensure that fresh bedrock is exposed and that the casing and annular grout seal are completely removed.

\subsubsection{Condition the Borehole}

Ream the borehole with a tri-cone drill bit that is at least 0.25 -in larger in diameter than the original borehole. This will expose fresh bedrock and help ensure an effective bond between the cement plug and the borehole wall.

\subsubsection{Set the Upper Plug}

Tremie cement, mixed to a density of 12 to 15 lbs/gal, from the top of the lower plug. If no surface/conductor casing is present, tremie the 
Y-12 PLANT GROUNDWATER PROTECTION PROGRAM

TITLE: Well Plugging and Abandonment Procedure
G-003

Rev. 1, October 1994 Page 13 of 18

cement to within $4 \mathrm{ft}$ of ground surface. If a surface/conductor casing is present, tremie the cement to within 4 ft of the bottom of the casing.

\subsubsection{Remove the Surface/Conductor Casing}

If the well was completed with surface/conductor casing, drill out the annular grout seal using a washover pipe advanced to the bottom of the casing. Retrieve the washover pipe and remove the casing. Using a tri-cone drill bit at least 0.25 -in larger in diameter than the original borehole, ream the borehole to the top of the existing cement plug. Tremie cement, mixed to a density of 12 to $15 \mathrm{lbs} / \mathrm{gal}$, from the top of the existing plug to within $4 \mathrm{ft}$ of the ground surface (or bottom of the conductor casing, if removing a surface casing).

It may be possible to remove PVC casing by drilling it out with a tri-cone drill bit. If this approach is feasible, the bit size must be at least 0.25 -in larger than the original borehole diameter to ensure that the casing and annular grout seal are completely removed.

\subsubsection{Verify Plug Depth}

Measure the depth to the top of the cement plug to verify that it is within $4 \mathrm{ft}$ of the ground surface. If not, add more cement until the specified depth is reached.

\subsubsection{Cap the Plug}

Fill the remainder of the borehole to ground surface with compacted non-contaminated soil.

\subsection{Method C}

Method $C$ is for wells constructed of 7-in-OD or smaller PVC well casing, and typically completed with 5 to 20-ft well screens and sand filter packs. Wells completed in bedrock may also have 8 to 12-in-OD steel or PVC surface casing extending from ground surface to the top of bedrock. Some wells may also be completed with a conductor casing. 


\section{Y-12 PLIANT GROUNDWATER PROTECTION PROGRAM}

G-003

Rev. I,

TITLE: Well Plugging and Abandonment Procedure

October 1994

Page 14 of 18

\subsubsection{Remove the Well Casing}

Drill out the annular grout seal around the well casing using a washover pipe advanced to the bottom of the borehole. Retrieve the washover pipe and remove the well casing. Staged removal of the casing string may be necessary if it cannot be removed in one operation.

It may be possible to remove the PVC well casing by drilling it out with a tri-cone drill bit. If this approach is feasible, the bit size must be at least 0.25 -in larger than the original borehole diameter to ensure that fresh bedrock is exposed (bedrock wells only) and that the casing and annular grout seal are completely removed.

\subsubsection{Condition the Borehole}

If the well was completed in bedrock, ream the borehole with a tri-cone drill bit that is at least 0.25 -in larger in diameter than the original borehole. This will expose fresh bedrock and help ensure an effective bond between the cement plug and the borehole wall.

\subsubsection{Set the Plug}

Tremie API Class A neat cement, mixed with potable water to a slurry density of 12 to $151 \mathrm{bs} / \mathrm{gal}$, from the bottom of the borehole. If no surface/conductor casing is present, tremie the cement to within $4 \mathrm{ft}$ of ground surface. If a surface/conductor casing is present, tremie the cement to within $4 \mathrm{ft}$ of the bottom of the casing.

\subsubsection{Remove the Surface/Conductor Casing}

If the well was completed with surface/conductor casing, drill out the annular grout seal using a washover pipe advanced to the bottom of the casing. Retrieve the washover pipe and remove the casing. Using a tri-cone drill bit at least 0.25 -in larger in diameter than the original borehole, ream the borehole to the top of the existing cement plug. Tremie cement, mixed to a density of 12 to $15 \mathrm{lbs} / \mathrm{gal}$, from the top of the existing plug to within 4 ft of the ground 


\section{Y-12 PLANT GROUNDWATER PROTECTION PROGRAM}

TITLE: Well Plugging and Abandonment Procedure
G-003

Rev. 1,

October 1994

Page 15 of 18

surface (or bottom of conductor casing if removing a surface casing).

It may be possible to remove PVC casing by arilling it out with a tri-cone drill bit. If this approach is feasible, the bit size must be at least 0.25 -in larger than the original borehole diameter to ensure that the casing and annular grout seal are completely removed.

\subsubsection{Verify Plug Depth}

Measure the depth to the top of the cement plug to verify that it is within $4 \mathrm{ft}$ of the ground surface. If not, add more cement until the specified depth is reached.

\subsubsection{Cap the Plug}

Fill the remainder of the borehole from the top of the cement plug to ground surface with compacted non-contaminated soil.

\subsection{Method D}

Method $D$ is for exploratory core holes constructed of 4.5-in-OD or smaller steel surface casing, which typically extends from ground surface into competent bedrock, with an open-hole interval below the bottom of the casing. The core holes may also have 8 to 12-in-OD steel or PVC conductor casing extending through the unconsolidated material.

\subsubsection{Condition the Open-Hole Interval}

The open-hole interval of the core holes will not be conditioned (i.e., fluid circulation or reamed to expose fresh bedrock). This would require the prior removal of the 4.5-in-OD surface casing, which may risk collapse of the upper portion of the core hole before P\&A operations are completed. In addition, core hole diameters are normally 3.5-in. or less, which are smaller than standard tri-cone bits.

\subsubsection{Set the Lower Plug}

Tremie API Class A neat cement, mixed with potable water to give a slurry density of 12 to 15 lbs/gal, from the bottom of the open-hole 
Y-12 PLANT GROUNDWATER PROTECTION PROGRAM

TITLE: Well Plugging and Abandonment Procedure
G-003

Rev. 1,

October 1994

Page 16 of 18

portion of the core hole. Because of the long open hole intervals in the core holes, the cement must be installed in stages of approximately 300 ft or less. During placement, tremie the cement from the bottom to the top of the particular interval being plugged, and allow the cement to set for 24 hours. In the final stage, tremie the cement to within $4 \mathrm{ft}$ of the bottom of the surface casing.

\subsubsection{Remove the Surface Casing}

Drill out the annular grout seal around the surface casing using a washover pipe advanced to the bottom of the casing. Retrieve the washover pipe and remove the casing. Staged removal of the casing may be necessary if it cannot be removed in one operation.

\subsubsection{Condition the Borehole}

Ream the borehole to the top of the existing cement plug using a tri-cone drill bit that is at least 0.25 larger than the original borehole diameter. This will expose fresh bedrock and help ensure an effective bond between the cement plug and the borehole well.

\subsubsection{Set the Upper PIug}

Tremie cement, mixed to a density of 12 to 15 lbs/gal, from the top of the lower plug. If no conductor casing is present, tremie the cement to within $4 \mathrm{ft}$ of ground surface. If a conductor casing is present, tremie the cement to within 4 ft of the bottom of the conductor casing.

\subsubsection{Remove the Conductor Casing}

If the core hole was completed with conductor casing, drill out the annular grout seal using a washover pipe advanced to the bottom of the conductor casing. Retrieve the washover pipe and remove the conductor casing. Using a tri-cone drill bit at least 0.25 -in larger in diameter than the original borehole, ream the upper portion of the borehole to the top of the existing cement plug. Tremie cement, mixed to a density of 12 to $15 \mathrm{lbs} / \mathrm{gal}$, from the top of the 
Y-12 PLANT GROUNDWATER PROTECTION PROGRAM

TITLE: Well Plugging and Abandonment Procedure
G-003

Rev. 1, October 1994 Page 17 of 18

\section{existing plug to within 4 ft of the ground surface. \\ It may be possible to remove PVC conductor casing by drilling it out with a tri-cone drill bit. If this approach is feasible, the bit size must be at least $0.25-i n$ larger than the original borehole diameter to ensure that the casing and annular grout seal are completely removed. \\ 8.6.7 Verify Plug Depth \\ Measure the depth to the top of the cement plug to verify that it is within 4 ft of the ground surface. If not, add more cement until the specified depth is reached. \\ 8.6.8 Cap the Plug}

Fill the remainder of the borehole to the ground surface with compacted non-contaminated soil.

\subsection{ACCEPTANCE CRITERIA}

The on-site geologist will verify that P\&A operations were performed in accordance with the specified method. Any deviations from the specified P\&A method must be pre-approved by the Y-12 Plant GWPPM or authorized designee. Requests for deviations may be verbal, but must be recorded immediately in the field log book and include date, time, and authorizing personnel. Deviations will also be noted on Activity/Progress Forms and P\&A Diagrams as appropriate.

\subsection{POST PERFORMANCE WORR ACTIVITIES}

10.1 The on-site geologist will submit the Well Plugging and Abandonment documentation to the Y-12 Plant GWPPM or authorized designee.

10.2 Waste materials generated during $P \& A$ will be disposed of in accordance with the Well Plugging and Abandonment Waste Management Plan. 
Y-12 PLANT GROUNDWATER PROTECTION PROGRAM |G-003

Rev. 1,

TITLE: Well Plugging and Abandonment Procedure fotober 1994

Page 18 of 18

\subsection{RECORDS}

11.1 Well Plugging and Abandonment Request Form

11.2 Well Plugging and Abandonment Waste Management Plan

11.3 Well Plugging and Abandonment Diagram

11.4 Well Plugging and Abandonment Activity/Progress Report 
C.2 WELL INSPECTION PROCEDURE 
Oak Ridge $Y-12$ Plant

Groundwater Protection Program

standard Practice Procedure

\author{
Fell Inspection Procedure \\ G-001 \\ Rev. 1., July 1994
}

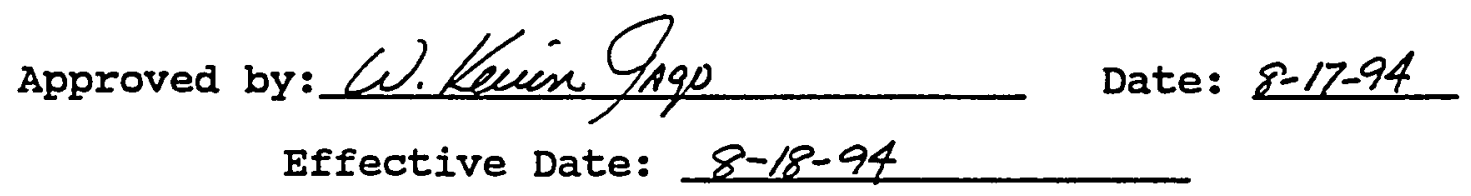

Record of Changes

\begin{tabular}{||c|c|c|c||c|c|c|c||}
\hline $\begin{array}{c}\text { Change } \\
\text { No. }\end{array}$ & $\begin{array}{c}\text { Affected } \\
\text { Pages }\end{array}$ & Approved Date & Expiration Date & $\begin{array}{c}\text { Change } \\
\text { No. }\end{array}$ & $\begin{array}{c}\text { Affected } \\
\text { Pages }\end{array}$ & $\begin{array}{c}\text { Approved } \\
\text { Date }\end{array}$ & $\begin{array}{c}\text { Expiration } \\
\text { Date }\end{array}$ \\
\hline \hline & & & & & & & \\
\hline & & & & & & & \\
\hline & & & & & & & \\
\hline
\end{tabular}

Next 3-year review required no later than: July 1997. 
Y-12 PIANT GROUNDWATER PROTECTION PROGRAM

TITLE:
Well Inspection Procedure
G-001

Rev. 1,

July 1994

Page 2 of 12

CONTENTS

Page

1.0 PURPOSE .................... 3

2.0 APPLICABILITY ................. 3

3.0 DEFINITIONS .................... 3

4.0 REFERENCES ................. 5

4.1 Use References . . . . . . . . . . . . . . 5

4.2 Source References .............. 6

5.0 PRECAUTIONS AND LIMITATIONS ............. 7

5.1 Annular Seal . . . . . . . . . . . . . 7

5.2 Constructed Well Depth ............. 7

5.3 Incrustation . . . . . . . . . . . . . 7

5.4 Limits of Tape Measure .............. 7

5.5 Measurement Accuracy . . . . . . . . . . . 7

5.6 Safety . . . . . . . . . . . ..... 7

5.7 Well Access .................. 8

6.0 PREREQUISITES . . . . . . . . . . . . . . . . . 8

6.1 Initial Inspection ................ 8

6.2 Subsequent Inspections . . . . . . . . . . . 8

7.0 TEST EQUIPMENT, TOOLS, AND SUPPIIES . . . . . . . . 8

$8.0^{\circ}$ ACTION STEPS ................... 9

8.1 Preparation . . . . . . . . . . . . . . 9

8.2 Inspection ................... 9

9.0 ACCEPTANCE CRITERIA . . . . . . . . . . . . . . 12

10.0 POST PERFORMANCE WORK ACTIVITIES . . . . . . . . . 12

10.1 Documentation . . . . . . . . . . . 12

10.2 Maintenance Work Inspection ............ 12

10.3 Plugging and Abandonment Requests ........ 12 


\subsection{PURPOBE}

This is a procedure intended to establish a systematic method for inspecting the physical condition of a monitor well and to identify monitor-well maintenance needs that will extend the life of the well and ensure the collection of representative groundwater quality samples and hydrologic data from the well.

\subsection{APPLICABILITY}

This procedure is applicable to all monitor wells located at the Y-12 Plant.

\subsection{DEFINITIONS}

Annular seal - a grout seal installed between the well casing and borehole wall or outer casing.

Christy Box - steel or plastic box installed below the ground surface that allows access to the top of casing in a flushmounted well design.

Concrete Pad - typically a neat cement or concrete pad at ground surface that surrounds the well casing or protective surface casing.

Constructed Depth - the distance from the top of the innermost well casing to the bottom of the screened or open interval as reported in: Updated Subsurface Data Base for Bear Creek Valley, Chestnut Ridge, and Parts of Bethel Valley on the U.S. Department of Energy Oak Ridge Reservation, Y/TS881 (R2), August 1994 (or most recent version).

Flush-Mounted Fell - well head completion where the top of casing is below the ground surface.

Groundwater Protection Program (GWPP) - a program developed per DOE Order 5400.1 to characterize the hydrogeology and monitor and protect groundwater quality at the Y-12 Plant.

GFPP Manager - person responsible for day-to-day management of the Y-12 Plant Groundwater Protection Program or authorized designee.

Guard Posts - posts placed around a well to prevent vehicular collision damage to the well. 
Y-12 PLANT GROUNDWATER PROTECTION PROGRAM G-001

Rev. 1, July 1994

TITLE: Well Inspection Procedure

Page 4 of 12

Hasp - a welded fastening that allows a well cap to be locked to the well casing, or a hinged steel lid to be locked to the protective casing.

Incrustation - deposition of mineral matter on the well screen and/or casing, typically through chemical or biological reactions.

Lock - a waterproof, steel or brass fastening device that secures the well cap or protective-casing lid and prevents unauthorized access to the well.

Measured Depth - the distance from the top of innermost well casing to the bottom of the well as measured in the field.

Monitor Well - a well installed to enable collection of groundwater samples and/or hydrologic data

(i.e., staticwater level).

Open-Hole Interval - a portion of a monitor well that contains no well casing through which groundwater enters the well and samples are obtained.

Primary Inspection Items - those components of a monitor well that are critical to the collection of representative groundwater quality samples and hydrologic information. Primary inspection items include the well casing and screen, hasp, lock, cap, well identification, and condition of the screened or open-hole interval.

Protective surface casing - a section of large-diameter steel pipe that is emplaced over the surface extension of a smaller diameter well casing to provide structural protection to the well and restrict unauthorized access to the well. A weep (hole) is usually located near the base of the casing to serve as a drain and prevent water from collecting inside the protective surface casing.

screened Interval - A portion of a monitor well that contains a slotted, perforated, or wire-wound section of casing (e.g., screen) through which groundwater enters the well and samples are obtained.

Secondary Inspection Items - those components of a monitor well which generally do not affect collection of representative groundwater quality samples or hydrologic information: these include well access, guard posts, and concrete pad. 
Y-12 PLANT GROUNDWATER PROTECTION PROGRAM

G-001

Rev. 1,

July 1994

Page 5 of 12

sediment Accumulation - accumulation of sand, silt, precipitates, or other debris in the bottom of the well.

Well Access - the means by which a well is accessible (e.g., gravel road).

Fell cap - a removable cap or hinged steel lid used to cover a well casing.

Tell casing - steel, stainless steel or PVC pipe which provides unobstructed access to the monitored interval.

Well Identification - a stainless steel plate that is engraved with the well identification number and is attached to the outermost casing.

\subsection{REFERENCES}

\subsection{Use References}

4.1.1 "Comprehensive Groundwater Monitoring Plan for the Department of Energy $\mathrm{Y}-12$ Plant Oak Ridge, Tennessee," Y/SUB/90-00206C/5, September 1990.

4.1.2 "Calendar Year 1993 Groundwater Quality Report for the Bear Creek Hydrogeologic Regime, Y-12 Plant, Oak Ridge, Tennessee," Y/SUB/94-EAQ10C/1, Parts 1 and 2 .

4.1.3 "Calendar Year 1993 Groundwater quality Report for the Chestnut Ridge Hydrogeologic Regime, $\mathrm{Y}-12$ Plant, Oak Ridge, Tennessee," Y/SUB/94-EAQ10C/3, Parts 1 and 2 .

1.1.4 "Calendar Year 1993 Groundwater Quality Report for the Upper East Fork Poplar Creek Hydrogeologic Regime, $\mathrm{Y}-12$ Plant, Oak Ridge, Tennessee," Y/SUB/94-EAQ10C/2, Parts 1 and 2 .

4.1.5 "Oak Ridge $Y-12$ Plant Groundwater Protection Program Management Plan (Revised),"

$Y / S U B / 93-Y P 507 C / 4$, June 1993 (or most recent revision).

4.1.6 "Updated Subsurface Data Base for Bear Creek Valley, Chestnut Ridge, and Parts of Bethel Valley on the U.S. Department of Energy Oak Ridge Reservation," Y/TS-88I(R2), August 1994 (or most recent revision. 
G-001

Rev. 1 , July 1994

Page 6 of 12

4.1.7 "Monitor Well Inspection and Maintenance Plan, Y-12 Plant, Oak Ridge, Tennessee (Revised)," Y/TS-1215, July 1994 .

\subsection{Bource References}

4.2.1 Aller, Ijinda, Truman W. Bennett, Gene Hackett, Rebecca J. Petty, Jay H. Lehr, Helen Sedoris, and David M. Nielsen "Handbook of Suggested Practices for the Design and Installation of Groundwater Monitoring Wells", NWWA, Dublin, Ohio, 398 p.

4.2.2 Driscoll, Fletcher G., 1986, "Groundwater and Wells", Johnson Division, st. Paul, Minnesota, 1089 p.

4.2.3 "Environmental Surveillance Procedures Quality Control Program," ESH/Sub/87/21706/1, February 1987.

4.2.4 Gass, Tyler E., Truman W. Bennett, James Miller and-Robin Miller, 1980, "Manual of Water Well Maintenance and Rehabilitation Technology", NWWA, Dublin, Ohio, $247 \mathrm{p}$.

4.2.5 Nielsen, David M., 1991, "Practical Handbook of Groundwater Monitoring", Lewis Publishers, Chelsea, Michigan, $717 \mathrm{p}$.

4.2.6 U.S. Department of Energy, "Procedures for the Collection and Preservation of Groundwater and Surface Water Samples and for the Installation of Monitoring Wells", GJ/TMC-08 (Second Edition) UC70A, October 1985 .

4.2.7 U.S. Environmental Protection Agency, "Environmental Compliance Branch standara operating Procedures and Quality Assurance Manual", Region IV, Athens, Georgia, February 1991.

4.2.8 U.S. Environmental Protection Agency, "RCRA Comprehensive Groundwater Monitoring Evaluation Document" (RCRA Groundwater Monitoring Systems), RCRA Enforcement Division, March 1988 .

4.2.9 U.S. Environmental Protection Agency, "RCRA Facility Investigation (RFI) Guidance, Volumes I-IV", OSWER Directive 9502.00-6C, July 1987. 
4.2.10 U.S. Environmental Protection Agency, "RCRA Groundwater Monitoring Technical Enforcement Guidance Document", OSWER-9950.1, September 1986.

\subsection{PRECAUTIONS AND IIMITATIONS}

\subsection{Annular seal}

The downhole condition of the annular seal cannot be determined without geophysical techniques. Such evaluation is beyond the scope of this procedure.

\subsection{Constructed Fell Depth}

The reported constructed depth of a well may require confirmation or may be inaccurate as recorded in original well construction records.

\subsection{Incrustation}

The downhole condition of a well screen cannot be determined without remote sensing. such evaluation is beyond the scope of this procedure.

\subsection{Iimits of Tape Measure}

Some wells are completed at depths (i.e., > $300 \mathrm{ft}$ ) that cannot be measured with a flat, weighted steel or fiberglass measuring tape. Additionally, the depth of wells which contain large water columns (i.e., greater than $100 \mathrm{ft}$ ) also may not be measurable with a flat, weighted measuring tape. A circular, stainless steel or coated steel measuring cable shall be used for all wells greater than a $300 \mathrm{ft}$ depth and is preferable for all wells.

\subsection{Measurement Accuracy}

Increased depth and large water columns decrease the accuracy of the well depth measurements.

\section{6 safety}

Established safety standards and requirements of Martin Marietta Corporation, DOE, and OSHA will apply to the inspection and maintenance of a monitor well. All field personnel will be provided with appropriate safety clothing, equipment, and training. 
Y-12 PLANT GROUNDWATER PROTECTION PROGRAM

TITLE: Well Inspection Procedure

G-001

Rev. 1,

July 1994

Page 8 of 12

\subsection{We11 Access}

A well may be deemed inaccessible because of site conditions or operations.

\subsection{PREREQUISITES}

\subsection{Initial Inspection}

If a well is currently scheduled for plugging and Abandonment $(P / A)$, inspection and maintenance of the well is not performed.

\subsection{Bubsequent Inspections}

If a well is currently included in the comprehensive groundwater monitoring program, the well is classified as active and a well inspection is performed annually. If not, the well is classified as inactive and $a$ well inspection is performed every three years. Wells for which the status changes from inactive to active will be inspected prior to monitoring.

\subsection{TEST EQUIPKENT, TOOLS AND SUPPLIES}

7.1 Documentation: Updated Subsurface Data Base (Y/TS-881/R2 or most recent revision), Well Inspection Maintenance Summary, Well Construction Data Summary, Well Inspection Checklist, Well Maintenance Request Form, Well Depth Measurement Procedure (G-002), Active Well status Checklist, and Daily Activity Log.

7.2 Field Equipment: Well locks, keys to unlock wells, weighted steel or fiberglass measuring tape and/or cable, pens, indelible markers, and clip-board.

7.3 Personal Protective Equipment:

Required: Rubber gloves

Optional: Safety shoes, tyvek coveralls, protective eye-wear, hard hat.

7.4 Decontamination Equipment: Plastic ground cover, distilled water, wash bottles, mild detergent, and collection vessels for wash and rinse water. 
Y-12 PLANT GROUNDWATER PROTECTION PROGRAM G-001

Rev. 1,

TITLE: Well Inspection Procedure

July 1994

Page 9 of 12

\subsection{ACTION 8TEPB}

\subsection{Preparation}

8.1.1 Identify wells to be inspected from the Well Inspection/Maintenance Summary.

8.1.2 Review Well Location Map(s) and Well Construction Data Summary to determine:

a. the well location:

b. the constructed depth of the well; and

c. length of the screen or open-hole interval for the well.

\subsection{Inspection}

8.2.1 On the Well Inspection Checklist, enter the inspection number for the well. The Y-12 Plant GWPP Manager or authorized designee will assign the inspection number using the following format: two-digit number denoting the year followed by a dash followed by a three-digit number (example: 91-001). Inspection numbers for each well should be assigned consecutively (i.e., 91-001, 91-002, $91-003, \ldots$.$) .$

Complete the Well Information section of checklist using information from the Updated Subsurface Data Base (for well number) and the Well Construction Data summary (for site, screened or open-hole interval length, and constructed well depth).

8.2.2 Verify that the monitor well is accessible by vehicle (active wells only). If construction, fencing, fallen trees, or site operation or closure activities have isolated the well, note on Well Inspection Checklist and report the finding to the Y-12 GWPP Manager or authorized designee. Otherwise, note any maintenance needs for well access road on Well Inspection Checklist.

8.2.3 Inspect guard posts for damage, physical deterioration, paint degradation, and proper positioning (active wells only). Each post should be painted high-traffic yellow, and be a height above ground that is adequate to prevent vehicular collision damage. The guard posts should be situated between the well and each 
Y-12 PIANT GROUNDWATER PROTECTION PROGRAM

TITLE: Well Inspection Procedure
G-001

Rev. 1,

July 1994

Page 10 of 12

traffic approach to the well. Complete appropriate section of Well Inspection Checklist.

8.2.4 Confirm that a stainless steel plate engraved with a legible well identification number is attached to the outermost casing of the monitor well. Through a comparison with the Updated Subsurface Data Base, confirm that the well number is correct. Complete appropriate section of Well Inspection checklist.

8.2.5 Inspect the concrete pad for cracks and deterioration (active wells only). The top of the pad should be level or slope away from the casing to prevent ponding of rain water around the well casing. Complete appropriate section of Well Inspection Checklist.

8.2.6 Inspect the lock for corrosion and operation of the locking mechanism. If a lock is corroded and difficult to open, replace it. Do not use any lubricant to improve lock performance. Complete appropriate section of Well Inspection Checklist.

8.2.7 Inspect the integrity of the hasps, making certain that they are firmly welded to the well cap and/or the metal casing. Complete appropriate section of Well Inspection Checklist.

8.2.8 Inspect the condition of the well cap or hinged steel lid. Complete appropriate section of Well Inspection Checklist.

8.2.9 Inspect all above-ground well casings and protective surface casings (if present) for cracks, corrosion, breaks, bends, or any other signs of deterioration that may effect structural integrity. Inspect base of protective surface casing to locate weep. Complete appropriate section of Well Inspection Checklist.

8.2.10 For flush-mounted wells, inspect traffic covers for presence of fasteners (bolts), excessive rust or deterioration, or any other notable damage. Covers should be securely bolted to the christy box.

8.2.11 For flush-mounted wells, inspect christy box for excessive rust or other damage. The concrete pad surrounding the christy box should be sloped as 
G-001

Rev. 1, July 1994

Page 11 of 12

to minimize the potential for water accumulation inside of the box.

8.2.12 For flush-mounted wells, inspect the water-tight well cap for tightness and condition of the rubber seal. Caps should fit securely so that they cannot be turned by hand.

8.2.13 Inspect the annular seal for cracks, if visible, and by shaking the well casing. The casing should not easily move. Complete appropriate section of Well Inspection Checklist.

8.2.14 Put on rubber gloves.

8.2.15 Remove lock and well cap.

8.2.16 Verify that an established reference mark (measuring point) is on the top of the innermost well casing. If not, establish a mark with indelible marker on the well casing for future reference and notify the Y-12 Plant GWPP Manager or authorized designee.

8.2.17 Measure the well depth from the established reference mark to the bottom of the well and record on the checklist to the nearest 0.1 foot. Perform measurement in accordance with $\mathrm{Y}-12$ Plant We11 Depth Measurement Procedure (G-002) .

8.2.18 Compare measured well depth to the constructed depth of the well by using the equation: Sediment Accumulation = Constructed Depth Measured Depth. The sediment accumulation divided by the screen or open-hole interval length must be less than 0.2 . complete appropriate section of Well Inspection Checklist.

8.2.19 If any shaded yes/no answer box for each item on Well Inspection Checklist is checked, complete Well Maintenance Request section of checklist noting if maintenance is needed for Primary or Secondary Inspection Item(s), or both.

Enter the maintenance request number for the well on the Well Inspection Checklist and Maintenance Request Form. The Y-12 Plant GWPP Manager or authorized designee will assign maintenance request numbers using the following format: a two-digit number denoting the year followed by a 
dash followed by a three-digit number with a "p" (for Primary Inspection Item), or " $S$ " (for Secondary Inspection Item), or "PS" (for both Primary and Secondary Inspection Items) suffix (examples: 91-001P, 91-001S, 91-001PS). Consecutive maintenance request numbers for each well should be assigned (example: 91-001P, 91002s, 91-003s,...).

8.2.20 Sign and date Well Inspection Checklist. .

\subsection{ACCEPTANCE CRITERIA}

If none of the inspection items require maintenance, inspection of the well is complete.

10.0 POST PERFORMANCE WORK ACTIVITIES

\subsection{Documentation}

Compile Well Inspection CheckIists and Well Maintenance Request Forms. Transfer appropriate data from checklists and forms to the Well Inspection/Maintenance Summary. Submit all checklists, forms, and the completed Well Inspection/Maintenance Summary to the $\mathrm{Y}-12$ Plant GWPP Manager or authorized designee.

\subsection{Maintenance Fork Inspection}

The Y-12 Plant GWPP Manager or authorized designee will schedule and coordinate all well maintenance activities. When requested maintenance has been completed, obtain original Well Maintenance Request Form from Y-12 Plant GWPP Manager or authorized designee and inspect maintenance work performed.

\subsection{Plugging and Abandonment Requests}

If the Y-12 Plant GWPP Manager or authorized designee determines that, based upon consultations with field inspection personnel and a well site visit (if needed), a Primary Inspection Item is damaged or deteriorated beyond practical repair, the well may require plugging and abandonment. The $\mathrm{Y}-12$ Plant GWPP Manager or authorized designee will prepare all Plugging and Abandonment Request Forms and schedule and coordinate all related activities. 


\section{C.3 WELL DEPTH MEASUREMENT PROCEDURE}


Oak Ridge Y-12 Plant Groundwater Protection Program standard Practice Procedure

Mell Depth Measurement Procedure

$$
\text { G-002 }
$$

Rev. 1., July 1994

Approved by: $G$ ). Kewin Pago Date: 8-17-94 Effective Date: $8-18-94$

Record of Changes

\begin{tabular}{||c|c|c|c|c|c|c|c|}
\hline $\begin{array}{c}\text { Change } \\
\text { No. }\end{array}$ & $\begin{array}{c}\text { Affected } \\
\text { Pages }\end{array}$ & $\begin{array}{c}\text { Approved } \\
\text { Date }\end{array}$ & $\begin{array}{c}\text { Expiration } \\
\text { Date }\end{array}$ & $\begin{array}{c}\text { Change } \\
\text { No. }\end{array}$ & $\begin{array}{c}\text { Affected } \\
\text { Pages }\end{array}$ & $\begin{array}{c}\text { Approved } \\
\text { Date }\end{array}$ & $\begin{array}{c}\text { Expiration } \\
\text { Date }\end{array}$ \\
\hline \hline & & & & & & & \\
\hline & & & & & & & \\
\hline & & & & & & & \\
\hline
\end{tabular}

Next 3-year review required no later than: July 1997 
Y-12 PLANT GROUNDWATER PROTECTION PROGRAM|G-002 Rev. 1,

TITLE: Monitor Well Depth Measurement Procedure

CONTENTS

Page

1.0 PURPOSE

2.0 APPLICABILITY

3. 0 DEFINITIONS

4.0 REFERENCES

4.1 Use References . . . . . . . . . . . . . . . . . 4

4.2 Source References . . . . . . . . . . . . . . . 4

5.0 PRECAUTIONS AND IIMITATIONS . . . . . . . . . . . . . . 4

5.1 Constructed Well Depth . . . . . . . . . . . . 4

5.2 Limits of Tape Measure ................. . 4

5.3 Measurement Accuracy . . . . . . . . . . . . 5

5.4 Safety ......... . . . . . . . . . . . 5

5.5 Well Access . . . . . . . . . . . . . . . 5

6.0 PREREQUISITES . . . . . . . . . . . . . . . . . . . . 5

7.0 TEST EQUIPMENT, TOOIS, AND SUPPIIES . . . . . . . . . . . 5

7.1 Documentation .................. . 5

7.2 Personnel Protection Equipment . . . . . . . . . . 5

7.3 Field Equipment ................... . . 6

7.4 Decontamination Equipment . . . . . . . . . . . 6

8.0 ACTION STEPS . . . . . . . . . . . . . . . . . . . 6

9.0 ACCEPTANCE CRITERIA • . . . . . . . . . . . . . . . . 6

10.0 POST PERFORMANCE WORK ACTIVITIES - . . . . . . . . . . . . 7

11.0 RECORDS . . . . . . . . . . . . . . . . . . . . 7 
Y-12 PLANT GROUNDWATER PROTECTION PROGRAM|G-002

Rev. 1,

TITLE: Monitor Well Depth Measurement Procedure July 1994

Page 3 of 7

\subsection{PURPOSE}

This procedure is a standardized method for determining the measured depth of a groundwater monitoring well. The measured depth of a well, when compared to the constructed depth of the well, provides an indication of sediment accumulation or obstructions within the well.

\subsection{APPLICABILITY}

Measuring well depth is applicable to all monitor wells located at the $\mathrm{Y}-12$ Plant.

\subsection{DEFINITIONS}

Constructed Depth - the distance from the top of the innermost well casing to the bottom of the screened or open interval as reported in: Updated Subsurface Data Base for Bear Creek Valley, Chestnut Ridge, and Parts of Bethel Valley on the U.S. Department of Energy Oak Ridge Reservation, Y/TS-881(R2), August 1994 (or most recent revision).

Groundwater Protection Program (GWPP) - a program developed per DOE Order 5400.1 to characterize the hydrogeology and monitor and protect groundwater quality at the Y-12 Plant.

GWPP Manager - person responsible for day-to-day management of the $\mathrm{Y}-12$ Plant Groundwater Protection Program or authorized designee.

Measured Depth - the distance from the top of the innermost well casing to the bottom of the well as measured in the field.

sediment Accumulation - accumulation of sand, silt, precipitates, or other debris in the bottom of the well.

Well cap - a removable cap used to cover a well casing.

Well Casing - steel, stainless steel, or PVC pipe which provides unobstructed access to the monitored interval.

Fell Identification - a steel plate embossed with the well identification number that is attached to the outermost casing. 
Y-12 PLANT GROUNDWATER PROTECTION PROGRAM|G-002

Rev. 1, TITLE: Monitor Well Depth Measurement Procedure

\subsection{Dge References}

4.1.1 "Updated Subsurface Data Base for Bear Creek Valley, Chestnut Ridge, and Parts of Bethel Valley on the U.S. Department of Energy Oak Ridge Reservation", Y/TS-881(R2), August 1994 (or most revision).

4.1.2 "Monitor-Well Inspection and Maintenance Plan, Y-12 Plant, Oak Ridge, Tennessee (Revised)", Y/TS-1215, JulY 1994

\subsection{Bource References}

4.2.1 Driscoll, Fletcher G., 1986," "Groundwater and Wells", Johnson Division, St. Paul, Minnesota, $1089 \mathrm{p}$.

4.2.2 "Environmental Surveillance Procedures Quality Control Program", ESH/Sub/87/21706/1, February 1987.

4.2.3 Gass, Tyler E., Truman W. Bennett, James Miller and Robin Miller, 1980, "Manual of Water Well Maintenance and Rehabilitation Technology", NWWA, Dublin, Ohio, 247 p.

4.2.4 U.S. Environmental Protection Agency, "A Compendium of superfund Field operations Methods", EPA/540/P-87/001, 1987.

4.2.5 U.S. Environmental Protection Agency, "RCRA Groundwater Monitoring Technical Enforcement Guidance Document", OSWER-9950.1, September 1986.

\subsection{PRECAUTIONS AND IIMITATIONS}

\subsection{Constructed Fell Depth}

The reported constructed depth of the well may require confirmation or may be inaccurate as recorded in original well construction records. 
Y-12 PLANT GROUNDWATER PROTECTION PROGRAM|G-002 Rev. 1, TITLE: Monitor Well Depth Measurement Procedure

\subsection{Limits of Tape Measure}

Some wells are completed at depths (i.e., > $300 \mathrm{ft}$ ) that cannot be measured with a flat, weighted steel or fiberglass measuring tape. Additionally, the depth of wells which contain large water columns (i.e., greater than $100 \mathrm{ft.}$ ) also may not be measurable with a flat, weighted measuring tape. A stainless steel or coated steel measuring cable shall be used for all wells greater than a 300-foot depth and is preferable for all wells.

\subsection{Measurement Accuracy}

Increased depth and large water columns decrease the accuracy of the well depth measurement.

\section{4 safety}

Established safety standards and requirements of Martin Marietta Corporation, DOE, and OSHA will apply to the process of obtaining the measured depth of a monitor well. All field personnel will be provided with appropriate safety clothing, equipment, and training.

\subsection{Tell Access}

A well may be deemed inaccessible because of site conditions or operations.

\subsection{PREREQUISITES}

All monitor wells will have the measured depth determined during a scheduled well inspection.

\subsection{TEST EQUIPMENT, TOOLS AND SUPPLIES}

7.1 Documentation: Well Construction Data Summary, Updated Subsurface Data Base, $Y / T S-881(R 2)$ (or most recent version), Daily Activity Logbook, and Monitor-Well Inspection Checklist.

7.2 Personnel Protection Equipment:

Required: rubber gloves

optional: safety shoes, tyvek coveralls, hard hat, and protective eye-wear. 
Y-12 PLANT GROUNDWATER PROTECTION PROGRAM|G-002 Rev. 1 , TITLE: Monitor Well Depth Measurement Procedure July 1994

7.3 Field Equipment: Keys to unlock wells, indelible marker, pen, clipboard,-and weighted fiberglass or steel measuring tape(s) and/or cable (the weight will be stainless steel or other approved inert material and have a blunt end facing down).

7.4 Decontamination Equipment: plastic ground cover, de-ionized water, mild detergent, and wash and rinse water collection vessels.

\subsection{ACTION STEPS}

8.1 Preparation: Review Well Construction Data summary and Updated Subsurface Data Base to obtain the constructed depth of the well and determine the well location.

8.2 Record well number and date.

8.3 Put on rubber gloves.

8.4 Remove well lock and well cap.

8.5 Locate the reference mark at the top of the innermost well casing. If a reference mark is not present, make one with indelible marker, and notify $Y-12$ Plant GWPP Manager or authorized designee.

8.6 Select the appropriate length measuring tape and/or cable.

8.7 Slowly lower the weight into the well until the bottom of the well is encountered as indicated by slack in the tape measure or a solid impact.

8.8 When slack or impact occurs, slowly lift the tape until the tape becomes taut. Raise and lower the tape until the point of tension release becomes clearly defined.

8.9 Hold the tape to the reference mark on the casing.

8.10 Record the measurement to the nearest 0.1 ft as the measured well depth in the Daily Activity Logbook and/or Well Inspection Checklist.

8.11 Repeat steps $8.6-8.9$ several times to ensure an accurate measurement. Readings should remain constant (i.e., within $0.1 \mathrm{ft}$ ). 
Y-12 PLANT GROUNDWATER PROTECTION PROGRAM|G-002

TITLE: Monitor Well Depth Measurement Procedure

Rev. 1,

July 1994

Page 7 of 7

8.12 Remove the measuring tape from the well and decontaminate in accordance with ESP-900.

8.13 Close well cap and replace lock.

9.0 ACCEPTANCE CRITERIA

An acceptable measured depth of a well is achieved when the range of three or more consecutive measurements are within $0.1 \mathrm{ft}$.

\subsection{POST-PERFORMANCE ACTIVITIES}

Report to the $\mathrm{Y}-12$ GWPP Manager or authorized designee those wells with significant (i.e., greater than 1 ft.) differences between the constructed well depth and the measured well depth.

11.0 RECORDS

11.1 Daily Activity Log

11.2 Well Inspection Checklist 


\section{APPENDIX D}

SAMPLING AND ENVIRONMENTAL SUPPORT DEPARTMENT OPERATING PROCEDURE - GROUNDWATER SAMPLING 


\section{GROUNDWATER SAMPLING SESD-TP-8204 REV. 0}

Written by: M.E. Cleveland

Subject Matter Expert: Fred Q. Nyen Date: $7-2.2-94$

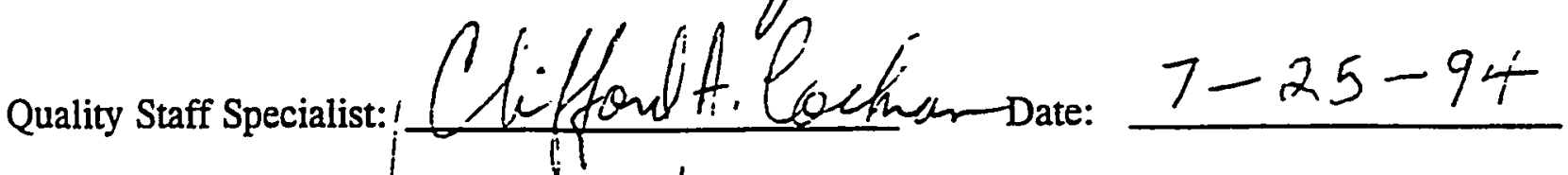
Approved by:
Effective Date: $1 / 25 / 94$
\begin{tabular}{|l|c|c|c|c|c|}
\hline \hline Procedure Type & Yes & No & Category & Yes & No \\
\hline In Hand & & $\checkmark$ & Safety System & & $\checkmark$ \\
\hline Upgraded & $\checkmark$ & & Quality-Related & & $\checkmark$ \\
\hline
\end{tabular}

Record of Changes

\begin{tabular}{||l|l|l|l|l||}
\hline Change No. & Affected Pages & $\begin{array}{c}\text { Approval } \\
\text { Signature }\end{array}$ & $\begin{array}{c}\text { Approval } \\
\text { Date }\end{array}$ & $\begin{array}{c}\text { Expiration } \\
\text { Date }\end{array}$ \\
\hline & & & & \\
\hline & & & & \\
\hline & & & & \\
\hline & & & & \\
\hline
\end{tabular}

Next review required no later than: $7 / 25 / 99$ 
K-25 Site Analytical Services Organization, Sampling and Environmental Support Department

TITLE: Groundwater Sampling
SESD-TP-8204

Rev. 0

Page 3 of 20

\section{CONTENTS}

Page

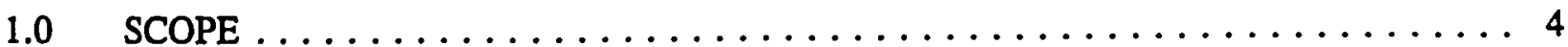

$2.0 \quad$ REFERENCED DOCUMENTS $\ldots \ldots \ldots \ldots \ldots \ldots \ldots \ldots \ldots \ldots \ldots$

$3.0 \quad$ RESPONSIBILITIES $\ldots \ldots \ldots \ldots \ldots \ldots \ldots \ldots \ldots \ldots \ldots \ldots \ldots$

4.0 TERMINOLLOGY $\ldots \ldots \ldots \ldots \ldots \ldots \ldots \ldots \ldots \ldots \ldots \ldots \ldots \ldots \ldots \ldots$

5.0 SUMMARY OF TEST METHODS $\ldots \ldots \ldots \ldots \ldots \ldots \ldots \ldots \ldots$

$6.0 \quad$ SIGNIFICANCE AND USE $\ldots \ldots \ldots \ldots \ldots \ldots \ldots \ldots \ldots \ldots \ldots$

$7.0 \quad$ INTERFERENCES $\ldots \ldots \ldots \ldots \ldots \ldots \ldots \ldots \ldots \ldots \ldots \ldots \ldots \ldots \ldots \ldots$

$8.0 \quad$ APPARATUS $\ldots \ldots \ldots \ldots \ldots \ldots \ldots \ldots \ldots \ldots \ldots \ldots \ldots \ldots$

9.0 REAGENTS AND MATERIALS $\ldots \ldots \ldots \ldots \ldots \ldots \ldots \ldots \ldots$

10.0 HAZARDS $\ldots \ldots \ldots \ldots \ldots \ldots \ldots \ldots \ldots \ldots \ldots \ldots \ldots \ldots$

11.0 SAMPLING, SUBSAMPLING, AND TEST SAMPLES $\ldots \ldots \ldots \ldots \ldots \ldots \ldots 11$

$12.0 \quad$ PREPARATION OF APPARATUS $\ldots \ldots \ldots \ldots \ldots \ldots \ldots \ldots \ldots \ldots \ldots \ldots \ldots \ldots$

13.0 CALIBRATION AND STANDARDIZATION $\ldots \ldots \ldots \ldots \ldots \ldots \ldots \ldots \ldots \ldots$

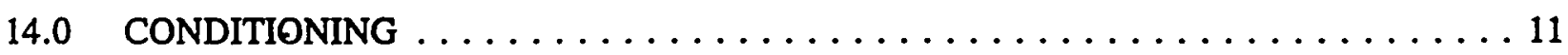

$15.0 \quad$ PROCEDURE $\ldots \ldots \ldots \ldots \ldots \ldots \ldots \ldots \ldots \ldots \ldots \ldots \ldots \ldots \ldots \ldots \ldots \ldots \ldots \ldots$

16.0 CALCULATION OR INTERPRETATION OF RESULTS $\ldots \ldots \ldots \ldots \ldots \ldots$

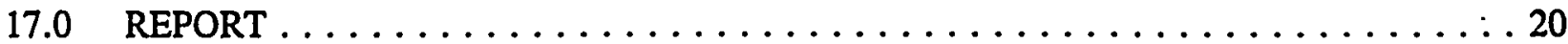

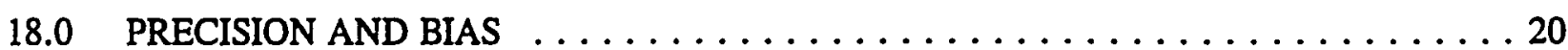

19.0 QUALITY ASSURANCE/QUALITY CONTROL $\ldots \ldots \ldots \ldots \ldots \ldots \ldots \ldots \ldots$

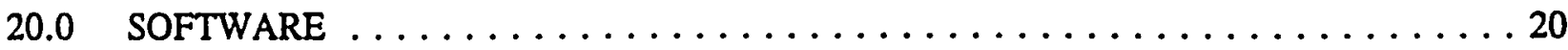


SESD-TP-8204

Rev. 0

Page 4 of 20
K-25 Site Analytical Services Organization, Sampling and Environmental Support Department

TITLE: Groundwater Sampling

\subsection{SCOPE}

This procedure describes the methods used to sample groundwater wells at the K-25 and Y-12 sites.

\subsection{REFERENCED DOCUMENTS}

2.1 U.S. Environmental Protection Agency, 1987, A Compendium of Superfund Field Operations Methods, EPA/540/P-87/001, Washington, D.C.

2.2 U.S. Environmental Protection Agency, 1986. Engineering Support Branch Standard Operating Procedures and Quality Assurance Manual, Region IV, Environmental Services Division, Atlanta, Ga.

2.3 U.S. Department of Energy, 1987, The Environmental Survey Manual, DOE/EH-0053.

2.4 U.S. Environmental Protection Agency, 1986, RCRA Groundwater Monitoring Technical Enforcement Document, OSWER-9950.1.

2.5 Martin Marietta Energy Systems, Inc., 1988, Environmental Surveillance Procedures Quality Control Program, Martin Marietta Energy Systems, ESH/Sub/87/21706/1, Oak Ridge, Tennessee.

2.6 U.S. Department of Transportation, Code FDR 49 Parts 100 - 177. Rev. 10-01-92.

\subsection{RESPONSIBILITIES}

\subsection{Sampling Supervisor}

3.1.1 Maintains communications with management and other groups for information that could affect the performance of this procedure.

3.1.2 Ensures that only trained field technicians are assigned to this procedure and that the training is documented.

3.1.3 Ensures that necessary materials and equipment are available and that maintenance is provided.

3.1.4 Schedules resources for sampling in conjunction with the ASO Project Manager.

3.1.5 Provides field sampling technicians with well data information, required sampling parameters, and sampling order as directed by customer.

3.1.6 Interfaces with the customer to obtain scheduling information, containment, access and information about unusual conditions.

3.1.7 Ensures quality in sampling through review of the technician's field sampling logbooks and field data sheets. 
3.1.8 Provides field quality assurance through planning and use of established and approved procedures.

3.1.9 Ensures complete chain-of-custody control through review of the chain-ofcustody documents.

3.1.10 Implements corrective actions for field QA deficiencies.

3.1.11 Provides notification of unusual field conditions to ASO Project Manager and the Groundwater Protection Program Manager (GPPM) at each site.

3.1.12 Maintains control of this procedure and its controlled copies.

\subsection{Sampling Technician}

3.2.1 Performs groundwater sampling according to this procedure only after receiving appropriate training.

3.2.2 Decontaminates and maintains sampling equipment as required by this procedure.

3.2.3 Legibly documents all field measurements, field conditions, and samples as required by this procedure and ensures that all field documentation is complete.

3.2.4 Takes samples as required by approved methodology and maintains sample quality at all times.

3.2.5 Properly preserves all samples according to EPA protocol as defined in the current and approved S/A Plan.

3.2.6 Delivers all samples to the K-25 ACD Sample Management Group custodian or to the contract laboratory under documented chain-of-custody within the required holding times.

3.2.7 Ensures a complete chain-of-custody documentation on all samples.

3.2.8 Maintains a clean and orderly working area in the truck or lab.

3.2.9 Conducts sampling operations in a safe, efficient manner so as to not injure themselves or others.

3.2.10 Maintains field copies of the Environmental Surveillance Procedures, SOPs, and the following items from the current S/A Plan: all addendas, quarterly sampling schedules, bottle lists, site maps, and well depths. 
SESD-TP-8204

Rev. 0

Page 6 of 20
K-25 Site Anaiytical Services Organization. Sampling and Environmental

Support Department

TITLE: Groundwater Sampling

\subsection{TERMINOLOGY}

Does not apply

\subsection{SUMMARY OF TEST METHODS}

5.1 Groundwater samples are collected after an appropriate well purge by using a bailer or pump. If required, the purge water from the wells is transferred into collection containers and is transported to the plant for appropriate water treatment. Field determinations which include $\mathrm{pH}$, specific conductance, oxidation-reduction potential, dissolved oxygen, and temperature are obtained on an initial sample from the well. Additional measurements of the same type are taken for each well volume purged and after the final purge volume. All samples are preserved in the field according to EPA protocol as defined in the current and approved Sampling and Analysis (S/A) Plan.

5.2 All samples are then delivered to the K-25 Analytical Chemistry Department (ACD), Sample Management Group (SMG), or to a contract laboratory.

\subsection{SIGNIFICANCE AND USE}

6.1 This method is applicable to the sampling of groundwater wells at K-25 and $\mathrm{Y}-12$ sites only.

6.2 This procedure does not replace manufacturer instrument operation manuals. The purpose of the procedure is to supplement that information and to provide field sampling technicians with additional sampling instructions.

\subsection{INTERFERENCES}

Does not appiy

\subsection{APPARATUS}

8.1 Safety equipment: Company issued clothing (including cold weather apparel, rain suits), pagers and cellular phones, steel-toed safety shoes, safety glasses with side shields, rubber gloves, if required Tyvek coveralls or rubber aprons, rubber overshoes, hard hat, cotton gloves, respirators, fire extinguishers, insect repellant, and insulated water coolers.

8.2 Consumables: Ground cloth (polyethylene), $\mathrm{pH}$ paper, fluorocarbon resin-coated wire, nylon cord, single strand stainless wire, tape, gloves, plastic bags, paper towels, wash bottles, and blue or water ice.

8.3 Field data sheets, chain-of-custody forms, and equipment calibration record forms; chainof-custody seals, labels, indelible black ink pens, indelible marking pens, and electronic calculator. 
K-25 Site Analytical Services Organization, Sampling and Environmental Support Deparment

8.4 Measuring tape and an electronic water level indicator (for measuring depth to water in the well).

8.5 Compressed gas cylinder or air compressor with associated regulators and high pressure air lines as necessary to operate the pump.

8.6 Bottom loading bailer of teflon or equivalent construction with a closed top, well sampling pump lines of sufficient length to reach the screened portion of the well, and if required controller boxes with associated air hoses and connections to operate the dedicated sampling pumps.

8.7 At the start of each day all field analytical equipment is calibrated in accordance with the procedures listed in Sections 8.7.1 through 8.7.5 of this procedure. Results are recorded in calibration $\log$ books with the sheet number recorded on the field data sheet. All temperature meters are calibrated and verified against a National Institute of Standards certified thermometer, which is calibrated annually by the K-25 Metrology, Testing and Equipment Laboratory (MT\&E). If an instrument is thought to be out of calibration; while in the field, the technician must recalibrate the instrument. If the instrument is found to be out of calibration; the supervisor must be contacted; so the customer and program management can determine if the previous data is valid; of if a resample is required. If an instrument is no calibrated on the proper schedule (overdue), the instrument will be treated as out of calibration and then will be tagged with a " Do Not Operate" tag. Immediate action should be taken to ensure the instrument is recalibrated.

\subsubsection{Temperature Meter Operation and Verification, SESD-TP-8005.R0, 11-29-93}

8.7.2 Ph Meter Operation and Calibration, SESD-TP-8007.R0, 11-19-93

8.7.3 Dissolved Oxygen Meter Operation \& Calibration, SESD-TP-8008.R0, 11-29-93

8.7.4 Redox Meter Operation and Calibration, SESD-TP-8201.R0, 11-29-93

8.7.5 Conductivity Meter Operation and Calibration, SESD-TP-8202.R0, 11-29-93

NOTE: Do not use balance if certification is expired.

8.8 Laboratory Anaiytical Balance must be certified annually by the K-25 Site MT\&E program and checked by technician each time of use to standard weight and recorded in reagent log book. Balance certification date must also be tracked in reagent log book.

8.9 Sample containers of assorted sizes and types as required by the analyses specified for each well as defined in the current and approved S/A Plan.

8.10 Filtration apparatus equipped with a 0.45 or 0.10 micron membrane filter for filtering the dissolved metals sample. 


\subsection{APPARATUS (Continued)}

8.11 Insulated ice chest containing $8 \mathrm{lbs}$. of ice or 8 pint size packs of "Blue Ice" or an equivalent amount for sample preservation.

8.12 Hunter/Keck - Model KIR-89 portable product level and thickness measuring device for inorganic layers, and the thickness of each layer in groundwater monitoring wells.

8.13 Tools and Tool Box.

8.14 Required Procedure Books and Training Manuals.

8.15 DOT approved packaging, labels, and shipping papers.

\subsection{REAGENTS AND MATERIALS}

9.1 ASTM Type II distilled deionized water. A carboy of the water will be collected and stored in K-1004-A, Room 18, with a conductivity reading recorded each time the carboy is filled and recorded in a reagent log book.

9.2 A Chemical Inventory Sheet (HMIS) is required for the Groundwater Sampling Group and is to be located in K-1004-A, Room 18, K-1004-H Dock, and each of the sampling vehicles. An "*" indicates those materials maintained in the sampling vehicle. A " $\dagger$ " denotes a DOT shipping label required for transfer.

NOTE: Visually inspect preservatives for discoloration or degradation before use to ensure reagent quality. Replace dispensing bottles January 1 and July 1 of each year.

NOTE: Replace standard solutions in each instrument box at the beginning of each quarter.

9.2.1 $\mathrm{H}_{2} \mathrm{SO}_{4}$ - Sulfuric Acid (96.4\%)

9.2.2 $\mathrm{HCl}-$ Hydrochloric Acid $(36.5-38.0 \%)$

9.2.3 $\mathrm{NaOH}$ - Sodium Hydroxide $(50.0 \%)$

9.2.4 $\mathrm{HNO}_{3}-\mathrm{Nitric}$ Acid

$(69.0-71.0 \%)$

9.2.5 Nitrogen, Compressed
UN 1830 PGII LID-QTY *†

UN 1789 PGII LTD-QTY *†

UN 1824 PGII LTD-QTY *†

UN 2031 PGII Corrosive *†

UN 1066 Nonflammable Gas labels applied *† 
9.2.7 Ascorbic Acid

9.2.8 Boit Wasp \& Hornet Killer II *

9.2.9 Buffer solutions 4,7, and 10 *

9.2.10 Formula 409

9.2.11 Hach Chlorine Equivalent Standard Solution

9.2.12 Isopropyl Alcohol

9.2.13 Lava Soap

9.2.14 Liqui-Nox Detergent

9.2.15 Micro Soap

9.2.16 Potassium chloride

9.2.17 Potassium ferricyanide

9.2.18 Potassium ferrocyanide

9.2.19 Sodium Calcium Hydrate (Soda Lime)

9.2.20 Sodium Sulfite *

9.2.21 Softcide Hand Wash

9.2.22 Zinc Acetate

9.2.23 $0.01 \mathrm{~N} \mathrm{KCl}$ standard solution. Each lot prepared and recorded in reagent log book.*

9.2.24 Zobell standard solution. Each lot prepared and recorded in reagent log book. *

9.2.25 Lead free gasoline *

9.2.26 10W-30W Motor Oil *

\subsection{HAZARDS}

10.1 Ensure that at least two persons are present during well sampling operations.

10.2 Conduct no sampling operations during thunderstorms. 
SESD-TP-8204

Rev. 0

Page 10 of 20
K-25 Site Analytical Services Organization, Sampling and Environmental Support Department

TITLE: Groundwater Sampling

\subsection{HAZARDS (Continued)}

10.3 Have some form of communication in the field for use during sampling operations.

10.4 Obtain permission to enter sampling areas located above the firing range and ensure that the firing range is clear before starting sampling operations.

10.5 On weekends or overtime hours, contact Plant Shift Superintendent at Y-12 and K-25 to give a record of sampling location.

10.6 Carry along an adequate supply of ASTM Type II distilled deionized reagent grade water for use in equipment rinses and cleaning.

10.7 Use adequate protective clothing during cold weather sampling operations. For hot weather sampling, carry an adequate supply of drinking water, insect repellent and wasp spray. For comfort, make sure air-conditioning in trucks is operational.

10.8 Recognize and be aware of hazardous flora and fauna.

10.9 Use the proper method for moving and lifting heavy equipment.

NOTE: Always remove the regulator and replace the cylinder cap before operating the vehicle.

10.10 Use proper technique for the safe operation of well pumps, portable gasoline-engine driven electric generators, portable air compressors, and high pressure compressed gas cylinders and regulators.

NOTE: Refer to Martin Marietta Energy Systems Safety and Health Plan (Y-12 procedure, 70 series for environmental health and safety applications).

10.11 Store, transport, and use hazardous and flammable reagents in the manner consistent with the guidance provided in each chemical's Material Safety Data Sheet (MSDS) and DOT regulations. This information can be found in Sections 8.14 and 9.0 of this procedure

10.12 Always wear steel-toed shoes, company issued clothing, gloves, and safety glasses while performing sampling operations.

10.13 As per the requirements of ESP-800, have all samples taken from K-25 and Y-12 groundwater wells, surveyed and green tagged by Health Physics before shipment to an offsite contract laboratory. Retain a copy of green tags for records. 
K-25 Site Analytical Services Organization, Sampling and Environmental

Supporr Department •

SESD-TP-8204

Rev. 0

TITLE: Groundwater Sampling

Page 11 of 20

\subsection{SAMPLING, SUBSAMPLING, AND TEST SAMPLES}

Does not apply

\subsection{PREPARATION OF APPARATUS}

Does not apply

\subsection{CALIBRATION AND STANDARDIZATION}

Calibrated instruments are used to take field measurements at the well sites as per Section 8.7.

\subsection{CONDITIONING}

Does not apply

\subsection{PROCEDURE}

\subsection{Preliminary Preparations}

15.1.1 Don the necessary safety equipment.

15.1.2 Clean all pumps, bailers, water level indicators, and other down-hole equipment.

15.1.3 Identify the well(s) to be sampled.

15.1.4 Obtain the required information about the specific well(s) to be sampled.

1. Well location

2. Depth of the well

3. Diameter of the well casing

4. Method(s) to be used to sample the well

15.1.5 Check the field instrumentation as per the Steps of Sections 8.7 and 13.0. Record the required information on the equipment calibration data form.

15.1.6 Prepare the required trip blanks according to the Sampling and Analysis Plan requirements and SESD-TP-8203.R0.

15.1.7 Load the vehicle with the required equipment and supplies and secure it for transportation of supplies to the field. 
SESD-TP-8204

Rev. 0

Page 12 of 20
K-25 Site Analytical Services Organization, Sampling and Environmental Support Department

TITLE: Groundwater Sampling

\subsection{Sampling}

15.2.1 Record the well number, site, date, weather conditions, arrival time, and other well specific information on the field data sheet. Examples would include problems with site access, equipment malfunctions, well obstructions, strange odors, names of visitors, etc.

NOTE: When the sampling equipment must be removed from the tailgate area, use plastic trays or groundcover to prevent possible contamination. Discard the plastic sheeting after use along with any other disposable items.

15.2.2 Keep all equipment in the truck bed during sampling.

15.2.3 Unlock and open the well; note the condition of the well.

15.2.4 Don rubber gloves.

NOTE: Change rubber gloves between sampling wells.

15.2.5 Remove any dust or corrosion from around the well head using a non-metal bound brush.

15.2.6 Sample the air in the well head for organic vapors if required by the current and approved S/A Plan.

15.2.7 Note the diameter of the well bore and locate the reference mark at the top of the well casing.

15.2.8 Check the battery on the water level indicator.

15.2.9 Lower the electronic water level indicator probe into the well, making sure that the cord on the probe does not scrape the sides of the well casing.

NOTE: Accurate well depth data will be provided by site GWPP Manager's office annually.

15.2.10 Measure the depth to water from the established reference mark at the top of the innermost well casing.

15.2.11 Stop lowering the probe when the buzzer sounds.

15.2.12 Pull up on the probe until the buzzer no longer sounds.

15.2.13 Lower the probe again slowly. 
K-25 Site Analytical Services Organization, Sampling and Environmental Support Department

15.2.14 Stop at the instant the buzzer sounds.

15.2.15 Hold the cord to the side of the casing at the location of the reference mark.

15.2.16 Mark the cord with your thumb where it touches the reference mark.

15.2.17 Use a measuring tape to determine the total depth from the top of the casing to the water if the cord does not have measurement graduations.

15.2.18 Record the value in the field data logbook to the nearest 0.01 foot as per ESP 302-1.

15.2.19 Determine the depth to water two additional times to verify correct measurement.

15.2.20 Record the additional values as described in steps 15.2.9 through 15.2.18.

15.2.21 Average the three depth to water measurements and record the average value in the field data logbook.

15.2.22 Remove the water level indicator from the well.

NOTE: If required by the current and approved S/A Plan, collect the rinse water from this cleaning procedure in an appropriate containment vessel, and properly dispose of the water at the wastewater treatment system at the plant.

15.2.23 Rinse the probe and any portion of the cable that contacted the well water with ASTM Type II distilled deionized reagent water.

15.2.24 Wipe the probe and cabling with paper toweis.

15.2.25 Place the indicator in a clean plastic bag to prevent contamination during transport.

15.2.26 Determine the height of the water column by subtracting the depth to the water from the total well depth.

15.2.27 Calculate the volume of water in the well casing using the appropriate formula given below. 


\title{
15.0 PROCEDURE (Continued)
}

\author{
$2^{\prime \prime}$ well: 0.1632 gallft $x$ (height of water column) = gallons \\ $4^{\prime \prime}$ well: 0.6528 gallft $x$ (height of water column) = gallons \\ $6^{\prime \prime}$ well: 1.4688 gallft $x$ (height of water column) = gallons
}

NOTE: Use a bailer that has a diameter of less than 2 " on all 2 " well casings.

15.2.28 Obtain an initial well water sample using either a bailer or a pump.

1. Attach the bailer to a fluorocarbon resin-coated wire, nylon cord, or single strand stainless steel wire.

2. Lower the bailer slowly to the mid-point of the screened or open portion of the well.

NOTE: The air can be connected prior to lowering the pump into well to insure that the pump head is working properly.

3. Lower the pump into the well casing to the air/water interface.

4. Attach an air line and source of compressed air to the pump.

NOTE: If required by the currently approved S/A Plan, collect the water from these analyses in an appropriate containment vessel. and properly dispose of the water at the plant wastewater treatment system.

5. Withdraw sufficient water from the well to perform the required "initial" determinations as specified in Step 15.2.30.4.

NOTE: If required by the currently approved S/A Plan, collect the well purging water in an appropriate containment vessel, and properly dispose of the water at the plant wastewater treatment system. 
15.2.29 Purge the well of the required number of volumes of water using a bailer (per ESP 302-3), or a gas driven Bennett Pump (per ESP 302-4), or using a Bladder pump (Well Wizard) (per ESP 302-5). Wells are required to be purged until at least three volumes of water are removed

NOTE: When evacuating low-yield wells( that are incapable of yielding three casing volumes), evacuate the well to dryness only once. A well is dry when the pump has been lowered to one foot from the bottom of the well and the water does not continue to evacuate.

1. Remove the required volumes of water or bail until the well goes dry if using a bailer.

2. Determine the pump flow rate in the field by timing the rate at which a one gallon container can be filled by the pump if using a pump.

3. Record the information on the field data sheet.

4. Calculate the required purge time based on this field determined pumping rate.

5. Purge the well a minimum of three well volumes of water or until the well goes dry.

NOTE: The purge rate of the Bennett Pump and Bladder pump is usually less than two gallons per minute.

6. Purge the well at a rate such that the recharge water does not become agitated or cascades back into the well.

7. Record the purge start time and the purge end time on the field data sheet.

15.2.30 Allow the water in the well to recover.

NOTE: Whenever full recovery exceeds two hours, extract the sample as soon as sufficient volume is available for a sample for each parameter. 
SESD-TP-8204

Rev. 0

Page 16 of 20
K-25 Site Analytical Services Organization, Sampling and Environmental Support Department

TITLE: Groundwater Sampling

\subsection{PROCEDURE (Continued)}

1. Recharge volume or time as directed by the current and approved S/A Plan.

2. Allow sufficient time for the well to recover and stabilize before obtaining samples if a well is bailed dry or pumped dry.

15.2.31 Sample the water in the well.

NOTE: Collect all samples at one time if sufficient water is present.

NOTE: Certain sites may require additional parameters (samples) at a site, such as COD, Cyanide, TPH. tritium, gamma spectrum, Ammonia, and Nitrite. See Bottle List for sampling sequence and additional parameters.

1. Obtain samples of the well water for the field determinations.

2. Obtain and analyze samples for $\mathrm{pH}$ as per ESP $307-2$, specific conductance, temperature as per ESP 307-1, oxidation-reduction potential as per ESP 307-5, and dissolved oxygen as per ESP 307-3.

3. Obtain separate analyses for each parameter at evenly spaced intervals during the purge cycle.

NOTE: When sampling VOA, TOX, and TOC from a dedicated bladder pump, cut the purge rate down to approximately $100 \mathrm{mi} / \mathrm{min}$. or as low as the pump will allow. When sampling VOA, TOX, and TOC with a bailer, lower the bailer slowly into the sample interval of the well to prevent loss of volatile components.

4. Collect the required samples for analysis in the laboratory.

- Collect and obtain sample per ESP 302-3 when using a bailer, per ESP 302-4 when using a Bennett pump, and per ESP 302-5 when using a bladder pump.

- Collect samples that may be affected by the loss of volatile components first, i.e., VOA, TOX, TOC.

- Collect VOA, TOX, and TOC samples using a bailer or Bladder pump.

- Transfer the samples slowly, with minimum agitation and aeration.

- Collect these samples with zero headspace and check to ensure the absence of air bubbles.

- Collect all VOAs, then all TOCs, then all the metals, etc. Do not pull one complete set of samples and then start to pull another set. 
- Collect samples for dissolved metals using an in-line filter while sampling with a Bennett pump or Bladder pump.

- If dissolved metal samples are being collected using a bailer, the unfiltered samples are returned to the laboratory for filtering and preservation.

- Collect the balance of the required samples for the particular site in the following order:

Base-Neutral-Acid Extractables
Herbicides
Pesticides
Phenols
Turbidity
Anions (Iodine, Carbonate-Bicarbonate, Nitrate-Nitrogen)
pH and conductivity
Total coliform bacteria
Total suspended solids
Total dissolved solids
Carbonate/Bicarbonate
Kjeldahl Nitrogen
Metals, total and dissolved
Gross alpha and beta
Total Radium
Uranium 235

- Exceptions to this sampling order include the following:

(A) The Bennett pump is used for purging and sampling groundwater wells located on the Y-12 and K-25 Plant sites. In these situations, the Bennett pump may be used to sample all non-volatile sample fractions first. Then remove the pump and collect the volatile fractions using a bailer. Note any exceptions to this order of collection in the field data sheets.

- Record the sample types taken on the field data sheet and the times when remaining samples are collected.

15.2.32 Obtain like samples one after another if duplicate samples are to be taken..

15.2.33 Add the required preservatives to the samples as they are obtained, if the preservatives have not already been added to the sample containers.

NOTE: Check the pH of all samples except the VOA samples in the $40 \mathrm{~mL}$ vials. 


\subsection{PROCEDURE (Continued)}

NOTE: Do not insert the $\mathrm{pH}$ paper into the sample. To ensure the proper amount of preservative was added, check the $\mathrm{pH}$ of the preserved sample with narrow range $\mathrm{pH}$ paper by pouring a small amount of the sample over the $\mathrm{pH}$ paper.

15.2.34 Adjust the $\mathrm{pH}$ of a sample to $<2.0$ by adding the appropriate acid for samples that require $\mathrm{pH}$ adjustment.

15.2.35 Document that the $\mathrm{pH}$ adjustment was made by checking the appropriate blank on the field data sheet.

15.2.36 Ensure that all samples are labeled properly with well number, date, time, sampler's name, any other pertinent information, and analysis.

NOTE: If required by the currently approved S/A Plan, collect the rinse water from this cleaning procedures in an appropriate containment vessel, and properly dispose of the water at the wastewater treatment system at the plant.

15.2.37 Wash the outside of the sample containers with distilled water.

15.2.38 Place the samples in a cooler containing blue or water ice.

15.2.39 Obtain any blank samples for the well as required by the Sampling and Analysis Plan.

15.2.40 Return the bailer to the lab for decontamination after each well's sampling is completed.

NOTE: If this is the last well for a given site, collect all equipment rinsate sample. Check the current S/A Plan for the analytical parameters required.

1. Rinse the interior and exterior with a non-phosphate detergent.

2 Rinse all detergent from the equipment with ASTM Type II distilled deionized reagent grade water.

3. Rinse the equipment a second time with ASTM Type II distilled deionized reagent grade water.

4. Rinse the bailers with a ten percent nitric acid solution, then with ASTM Type II distilled deionized reagent grade water.

5. Wipe down the equipment with paper toweling.

6. Wrap fully assembled bailer in aluminum foil for lab to field transport. 
Field clean the Bennett pumps after sampling each well in a given site as described below.

1. Rinse the entire length of the pump head with water, then pump one to two gallons of a non-phosphate detergent water through the system.

2. Rinse all detergent from the pump by flushing the entire length of the pump head with one to two gallons of ASTM Type II distilled deionized reagent grade water.

3. Rinse the equipment a second time with an additional one to two gallons of ASTM Type II distilled deionized reagent grade water.

4. Seal the pump head in a clean plastic bag and proceed to the next well site for sampling.

15,2.42 Decontaminate the Bennett pump after sampling the site (one to ten wells) by taking the pump to the lab area where a steam supply is available.

1. Steam clean the pump head and the entire length of tubing.

2. Bring pump into lab to perform rinse.

NOTE: . To minimize waste, use approximately 0.7 gallons of solution for the 125 foot pump; approximately 1.4 gallons for the 250 foot pump; and approximately 2.8 gallons for the 500 foot pump.

3. Run a rinse solution consisting of $10 \%$ Nitric acid and water in lab ensuring that entire tubing bundle of the pump is fully filled with the solution.

4. Collect the Nitric acid waste in 55-gallon Acid Waste Drum.

5. Run a 30 minute tap water rinse to thoroughly remove the Nitric acid and any remaining residue.

6. Flush the tubing and pump with two tubing volumes of ASTM Type II deionized, distilled reagent grade water.

7. Wipe down pump head with paper towel and wrap in aluminum foil for lab to field transport.

8. Collect an equipment rinse blank at the frequency and for the parameters specified in the current and approved S!A Plan. 


\subsection{PROCEDURE (Continued)}

15.2.43 Replace the cover on the well and secure the well with the lock.

15.2.44 Return the sampling equipment to the vehicle and secure the equipment for transport back to the plant or to the next well site.

15.2.45 Clean up the well sampling site.

15.2.46 Enter the departure time in the field data sheet.

15.2.47 Report any maintenance problems at the well site (such as replacing hasps, locks, concrete pads, protective posts, obstructions inside of the well casings, well access, etc.) to the appropriate GPPM by means of a Well Maintenance Request Form.

15.2.48 Sign the field data sheet.

\subsection{CALCULATION OR INTERPRETATION OF RESULTS}

The volume of water in a well casing is calculated using the formulas listed in step 15.2.26. The purge time and pump flow rate is calculated according to the step 15.2.28.2.

\subsection{REPORT}

All sampling data collected from a well site is recorded on the field data sheet and in the field data logbook.

\subsection{PRECISION AND BIAS}

Does not apply

\subsection{QUALITY ASSURANCE/QUALITY CONTROL}

19.1 Well sampling frequency and the required analyses are determined by the well location and the current Sampling and Analysis Plan.

19.2 This procedure will be performed in accordance with Martin Marietta Energy Systems regulations and state and federal laws.

\subsection{SOFTWARE}

Does not apply 
APPENDIX E

FALSE-POSITIVE DEMONSTRATIONS 


\title{
FALSE-POSITTVE DEMONSTRATION FOR CHESTNUT RIDGE SEDIMENT DISPOSAL BASIN \\ AND KERR HOLLOW QUARRY
}

OAK RIDGE Y-12 PLANT

\author{
C. Stephen Haase ${ }^{1}$ \\ Helen L. King 2 \\ Terre M. Mercier ${ }^{3}$
}

September 10, 1991

1 Environmental Sciences Division, Oak Ridge National Laboratory, P. O. Box 2008, Oak Ridge, Tennessee 37831-6352

2 Environmental Management Department, Oak Ridge Y-12 Plant, P. O. Box 2009, Oak Ridge, Tennessee 37831-8219

3 H \& R Technical Associates, Oak Ridge, Tennessee 37830 


\section{Introduction}

Groundwater quality data were collect for two sites in RCRA Interim Status Detection Monitoring at the Oak Ridge $Y-12$ Plant during the first quarter of calendar year (CY) 1991. As required by Rule 1200-1-1-.05(6) of the Tennessee Department of Environment and Conservation, monitoring results for indicator parameters $(\mathrm{pH}$, conductivity, total organic carbon (TOC), and total organic halides (TOX)) were reviewed to determine if a statistically significant change had occurred in the values of one or more indicator parameters. Anatysis of the data obtained for the first quarter of 1991 indicated that statistically significant changes had occurred in $\mathrm{pH}$ for upgradient and downgradient wells at the Chestnut Ridge Sediment Disposal Basin and in $\mathrm{pH}$ and conductivity for upgradient and downgradient wells at Kerr Hollow Quarty.

The purpose of this document is to review $\mathrm{pH}$ and conductivity data in question to determine if the statistically significant changes in the indicator parameters noted for first-quarter 1991 data represent a release of contaminants into the groundwater from the facilities, or if the changes observed are due to other, naturally occurring factors.

\section{Chestnut Ridge Sediment Disposal Basin}

\section{Data Summary}

Data obtained during the first-quarter of 1991 indicate that statistically significant changes in $\mathrm{pH}$ occurred for both upgradient and downgradient monitoring wells at the Chestnut Ridge Sediment Disposal Basin. To evaluate these findings, all pH data from groundwater monitoring wells surrounding the facility (Fig. 1) have been reviewed to determine if the $\mathrm{pH}$ changes observed are truly indicative of contaminant releases from the Chestnut Ridge Sediment Disposal Basin.

\section{Evaluation of $\mathrm{pH}$ Data}

The mean, median, and variance of $\mathrm{pH}$ measurements obtained for all groundwater monitoring wells at the Chestnut Ridge Sediment Disposal Basin During are illustrated in Fig. 2. The data indicate that (1) the median $\mathrm{pH}$ values for all wells except well 1096 fall between 7.0 and 8.0 and (2) the degree of variance, as calculated by the distance between the 25th and 75th percentiles, is variable from well to well and ranges from approximately $0.5 \mathrm{pH}$ units for wells GW-156 and GW-159 to approximately $2.0 \mathrm{pH}$ units in well GW-158. 
Skewness and kurtosis values for the data sets for each well were evaluated to determine if the $\mathrm{pH}$ values followed a normal distribution. Such an analysis suggests that $\mathrm{pH}$ values in four out of ten wells at the site may not be normally distributed. Of importance to detection monitoring requirements, the skewness and kurtosis values suggest that $\mathrm{pH}$ values for upgradient well $\mathrm{GW}-159$, and downgradient wells GW-155 and GW-156 may not be normally distributed.

Trend plots for pH measurements from the upgradient and downgradient wells at the Chestnut Ridge Sediment Disposal Basin are illustrated in Figs. 3 through 6. Data for each of the wells will be summarfied and interpreted in the following sections.

GW-159. Upgradient well GW-159 had $\mathrm{pH}$ values $>8.0$ for the base year of CY 1986 (Fig. 3). Subsequent to the base year, all $\mathrm{pH}$ values from well $\mathrm{GW}-159$ were less than those observed during the base year, with values ranging from slightly above 8.0 to slightly less than 6.0. After the base year, $\mathrm{pH}$ values for well GW-159 exhibit a sawtooth cyclic variation. Values for $\mathrm{pH}$ gradually decline from approximately 8.0 to 6.0 during the period 1987 through mid-1989. During late $C Y 1989, \mathrm{pH}$ values increase back to approximately 8.0 and mark the beginning of a second sawtooth cycle. Subsequent to this abrupt increase, $\mathrm{pH}$ values exhibit a gradual decline from approximately 8.0 to 7.0 during $C Y 1990$. The $\mathrm{pH}$ value for first quarter 1991 is 7.6, which is below the median of 7.8, but within the interquartile range of 7.4 to 8.1 for the well (see Figs. 2 and 3 ), and within the maximum range of $\mathrm{pH}$ values observed in the well (see Fig. 3).

GW-155. Downgradient well GW-155 had $\mathrm{pH}$ values between slightly greater than 8.0 and approximately 7.5 during the base year of CY 1986 (Fig. 4). After the base year, $\mathrm{pH}$ values for well GW-155 exhibit a sawtooth cyclic variation similar to that observed in the upgradient well GW-159. Values for $\mathrm{pH}$ gradually decline from approximately 7.5 to slightly less than 6.0 during the period 1987 through mid-1989. During late CY 1989, $\mathrm{pH}$ values increase back to approximately 8.0, and mark the beginning of a second sawtooth cycle. Subsequent to this abrupt increase, $\mathrm{pH}$ values exhibit a gradual decline from approximately 8.0 to 7.0 during $C Y 1990$. In general pattern of pH fluctuations in well GW-155 is similar to that observed in the upgradient well. The $\mathrm{pH}$ value for first quarter 1991 is 6.9, which is below the median of 7.5, but within the interquartile range of 6.9 to 7.7 for the well (see Figs. 2 and 4), and within the maximum range of $\mathrm{pH}$ values observed in the well (see Fig. 4).

GW-156. Downgradient well GW-156 had pH values between slightly less than 9.0 and approximately 8.0 during the base year of CY 1986 (Fig. 5). Subsequent to the base year, $\mathrm{pH}$ values for well GW-156 were typically less than those obtained during the base year, with values ranging between approximately 8.0 and slightly less than 7.0. Values for $\mathrm{pH}$ gradually decline from approximately 7.8 to slightly less than 7.0 during the period 1987 through mid-1989. During CY 1990, $\mathrm{pH}$ values have increased somewhat, and range between approximately 7.8 and 7.3. The general 
pattern of pH fluctuations in well GW-156 is similar to that observed in the upgradient well (GW-159), although the sawtooth pattern is subdued due to the smaller range of $\mathrm{pH}$ fluctuation exhibited by well $\mathrm{GW}-156$. The $\mathrm{pH}$ value for first quarter 1991 is 7.3, which is below the median of 7.5, but within the interquartile range of 7.3 to 7.7 for the well (see Figs. 2 and 5), and within the maximum range of $\mathrm{pH}$ values observed in the well (see Fig. 5).

GW-157. Downgradient well GW-157 had $\mathrm{pH}$ values between approximately 7.5 and approximately 8.5 during the base year of CY 1986 (Fig. 6). Subsequent to the base year, $\mathrm{pH}$ values for well $\mathrm{GW}-157$ were typically less than those obtained during the base year, with values ranging between approximately 7.5 and slightly less than 7.0. Values for $\mathrm{pH}$ gradually declined from approximately 7.5 to slightly less than 7.0 during the period 1987 through mid-1989. During the last part of CY 1989, pH values have increased from approximately 7.0 to greater than approximately 7.5 , and range between approximately 7.5 and 7.8. The general pattern of $\mathrm{pH}$ fluctuations in well GW-157 is similar to that observed in the upgradient well (GW-159), although the sawtooth pattern is subdued due to the smaller range of $\mathrm{pH}$ fluctuation exhibited by well GW-157. The pH value for first quarter 1991 is 7.4, which is at the median of 7.4, within the interquartile range of 7.2 to 7.5 for the well (see Figs. 2 and 6), and within the maximum range of $\mathrm{pH}$ values observed in the well (see Fig. 6).

\section{Discussion and Summary}

Temporal variations in downgradient wells at the Chestnut Ridge Sediment Disposal Basin are generally similar to those exhibited by the upgradient well. The temporal $\mathrm{pH}$ trend of the upgradient well is interpreted to represent naturallyoccurring, secular pH fluctuations of the groundwater system surrounding the Chestnut Ridge Sediment Disposal Basin. All of the downgradient wells exhibit similar trends to that observed in the upgradient well. The temporal $\mathrm{pH}$ trend for well GW-155 is essentially identical to that observed in the upgradient well. The pH trends exhibited in downgradient wells GW-156 and GW-157 have similar form but differ in the magnitude of the the range of $\mathrm{pH}$ values observed. The similarity of trends suggests that variations in $\mathrm{pH}$ for groundwaters from all wells at the Chestnut Ridge Sediment Disposal Basin are due to secular variations in groundwater chemistry and not to contaminant releases from the site.

The $\mathrm{pH}$ values obtained during the base year of 1986 for the upgradient well and two of the three downgradient wells are always above 8.0, and typically above 9.0. Such values are significantly higher than those typical of uncontaminanted groundwater from the Knox Group studied elsewhere on the Oak Ridge Reservation (HSW 1991a through e). Such values are interpreted to represent partial contamination of the groundwater samples by fluids remaining in the formation from well construction activities. All of the groundwater monitoring wells at the Chestnut Ridge Sediment Disposal Basin were developed after construction, and purged prior to sampling. However, the fractured and karstified nature of the Knox aquifer makes 
development of wells potentially problematical. Because of such complications, and the fact that $\mathrm{pH}$ values for all wells during subsequent years were within ranges typical of Know Group. groundwaters elsewhere in the Oak Ridge area, data obtained during the base period are suspect.

Based on the factors discussed above, the statistically significant changes in pH noted for wells at the Chestnut Ridge Sediment Disposal Basin are interpreted to be false positives, and to not represent a release of contaminants into groundwater from the site.$$
=
$$ \\ Kerr Hollow Quarry
}

\title{
Data Summary
}

Data obtained during the first-quarter of 1991 indicate that statistically significant changes in $\mathrm{pH}$ were noted for the upgradient well (GW-145) and all three downgradient monitoring wells (GW-142, GW-144, and GW-231) at Kerr Hollow Quarry (Fig. 7). Additionally, statistically significant increases in conductivity for one downgradient well (GW-142) were noted. To evaluate these findings, all $\mathrm{pH}$ and conductivity data from groundwater monitoring wells surrounding the facility have been reviewed to determine if the changes observed are truly indicative of contaminant releases from Kerr Hollow Quarry.

\section{Evaluation of $\mathrm{pH}$. Data}

The mean, median, and variance of $\mathrm{pH}$ measurements obtained for all groundwater monitoring wells at Kerr Hollow Quarry are illustrated in Fig. 8. The data indicate that (1) the median $\mathrm{pH}$ values for all wells fall between 7.5 and 8.0 and (2) the variability or spread of the middle 50 percent of the data for each well, as illustrated by the interquartile distance (distance between the 25th and 75th percentiles), is variable from well to well and ranges from approximately $0.5 \mathrm{pH}$ units for well GW231 to approximately $2.0 \mathrm{pH}$ units in well GW-146.

Skewness and kurtosis values for the data sets for each well were evaluated to determine if the $\mathrm{pH}$ values followed a normal distribution. Such an analysis suggests that $\mathrm{pH}$ values in four out of seven wells at the site may not be normally distributed. Of importance to detection monitoring requirements, the skewness and kurtosis values suggest that $\mathrm{pH}$ values in downgradient wells $\mathrm{GW}-144$ and $\mathrm{GW}-231$ may not be normally distributed. .

Trend plots for $\mathrm{pH}$ measurements from the upgradient and downgradient wells at Kerr Hollow Quarry are illustrated in Figs. 9 through 12. Data for each of the wells will be summarized and interpreted in the following sections 
GW-145. Upgradient well $\mathrm{GW}-145$ had $\mathrm{pH}$ values ranging between approximately 7.9 and 8.7 for the base year of CY 1986 (Fig. 9). Subsequent to the base year, all pH values from well GW-145 were typically less than 8.5, with values ranging from slightly above 8.3 to approximately 6.6. After the base year, $\mathrm{pH}$ values for well GW145 exhibit a generalized decline through during the period 1987 through 1989, although the trend of this decline in values is complex and exhibits several reversals. This period of decreasing $\mathrm{pH}$ values concludes in late $\mathrm{CY} 1989$, when $\mathrm{pH}$ values of approximately 6.6 are measured. Such values are the lowest values obtained during the period of observation. Fie $\mathrm{pH}$ value from the sample obtained in late in CY 1989 exhibits an abrupt increase back to approximately 8.0. Subsequent to this abrupt increase, $\mathrm{pH}$ values during CY 1990 exhibit a gradual decline that is again characterized by much variability and at lease one reversal of trend. The $\mathrm{pH}$ value for first quarter 1991 is 7.1, which is below the median of 7.9, but within the interquartile range of 7.3 to 8.2 for the well (see Figs. 8 and 9), and within the maximum range of $\mathrm{pH}$ values observed in the well (see Fig. 9).

$G W-142$. Downgradient well GW-142 had $\mathrm{pH}$ values ranging between approximately 8.8 and 7.5 for the base year of CY 1986 (Fig. 10). Subsequent to the base year, all $\mathrm{pH}$ values from well GW-145 were typically less than 8.0 , with values ranging from approximately 7.9 to 6.6 . Values for $\mathrm{pH}$ from well GW-142 exhibit a generalized decline through 1989, although the trend of this decline in values is complex and exhibits several reversals. This period of decreasing $\mathrm{pH}$ values ends in late CY 1989, when $\mathrm{pH}$ values of approximately 6.6 are obtained. Such values are the lowest values obtained during the period of observation. The $\mathrm{pH}$ value from the sample obtained in late in CY 1989 exhibits an abrupt increase back to approximately 8.0. Subsequent to this abrupt increase, $\mathrm{pH}$ values during CY 1990 exhibit a gradual decline that is again characterized by much variability and at lease one reversal of trend. The pH value for first quarter 1991 is 7.1, which is below the median of 7.5, but within the interquartile range of 7.1 to 7.8 for the well (see Figs. 8 and 10), and within the maximum range of $\mathrm{pH}$ values observed in the well (see Fig. 10). In general, the trend of $\mathrm{pH}$ values for well GW-142 resembles that observed in upgradient well GW-145.

GW-144. Downgradient well GW-144 had pH values between approximately 8.0 to 7.8 during the base year of CY 1986 (Fig. 11). Subsequent to the base year, all pH values from well GW-144 were typically less than 8.0, with values ranging from approximately 7.9 to 6.5 . Values for $\mathrm{pH}$ from well GW-144 exhibit a generalized decline through 1989, although the trend of this decline in values is complex and exhibits several reversals. This period of decreasing $\mathrm{pH}$ values ends in late CY 1989, when $\mathrm{pH}$ values of approximately 6.5 are obtained. Such values are the lowest values obtained during the period of observation. The $\mathrm{pH}$ value from the sample obtained in late in CY 1989 exhibits an abrupt increase back to approximately 7.7. Subsequent to this abrupt increase, $\mathrm{pH}$ values during CY 1990 exhibit a gradual decline that is again characterized by much variability and at least one reversal of trend. The $\mathrm{pH}$ value for first quarter 1991 is 6.9, which is below the median of 7.6, 
but within the interquartile range of 7.6 to 7.9 for the well (see Figs. 8 and 11), and within the maximum range of $\mathrm{pH}$ values observed in the well (see Fig. 11). In general, the trend of $\mathrm{pH}$ values for well $\mathrm{GW}-144$ resembles that observed in upgradient well GW-145.

GW-231. Downgradient well GW-231 had $\mathrm{pH}$ values between approximately 8.1 and 7.6 during the base year of CY 1986 (Fig. 12). Subsequent to the base year, all pH values from well GW-231 were typically less than 7.5 , with values ranging from approximately 7.5 to 6.3 . Values for $\mathrm{pH}$ from well GW-231 exhibit a generalized decline through 1989, although the trend ofhis decline in values is complex and exhibits several reversals. This period of decreasing $\mathrm{pH}$ values ends in late CY 1989, when $\mathrm{pH}$ values of approximately 6.3 are obtained. Such values are the lowest values obtained during the period of observation. The $\mathrm{pH}$ value from the sample obtained in late in CY 1989 exhibits an abrupt increase back to approximately 7.7. Subsequent to this abrupt increase, pH values during CY 1990 exhibit a gradual decline that is again characterized by much variability and at lease one reversal of trend. The pH value for first quarter 1991 is 6.9, which is below the median of 7.5, below the interquartile range of 7.0 to 7.6 for the well (see Figs. 8 and 12), but within the maximum range of $\mathrm{pH}$ values observed in the well (see Fig. 12). In general, the trend of $\mathrm{pH}$ values for well GW-231 resembles that observed in upgradient well GW-145.

\section{Evaluation of Conductivity Data}

The mean, median, and variance of conductivity measurements obtained for all groundwater monitoring wells at Kerr Hollow Quarry are illustrated in Fig. 13. The data indicate that (1) the median conductivity values for all wells fall between approximately 250 and $500 \mu \mathrm{mhos} / \mathrm{cm}$ and (2) the variability or range of the middle 50 percent of the data for each well, as illustrated by the interquartile distance, is variable from well to well and ranges from approximately $75 \mu \mathrm{mhos} / \mathrm{cm}$ in well GW-231 to approximately $200 \mu \mathrm{mhos} / \mathrm{cm}$ in well GW-146.

Skewness and kurtosis values for the data sets for each well were evaluated to determine if the conductivity values followed a normal distribution. Such an analysis suggests that conductivity values in five out of seven wells at the site may not be normally distributed. Of importance to detection monitoring requirements, the skewness and kurtosis values suggest that conductivity values in upgradient well GW-145 and in downgradient wells GW-144 and GW-231 may not be normally distributed.

Trend plots for conductivity measurements from the upgradient well (GW-145) and the downgradient well (GW-142) of interest at Kerr Hollow Quarry are illustrated in Figs. 14 through 15. Data for each of the wells will be summarized and interpreted in the following sections 
GW-145. Upgradient well GW-145 had conductivity values ranging between: approximately 650 and $550 \mu$ mhos/cm for the base year of CY 1986 (Fig. 14). Subsequent to the base year, all conductivity values from well GW-145 were less than $600 \mu \mathrm{mhos} / \mathrm{cm}$, and ranged from approximately 560 to $375 \mu \mathrm{mhos} / \mathrm{cm}$. Conductivity values gradually decline during the period 1987 through 1990 , although the trend of this decline in values is complex and exhibits several reversals. The conductivity value for first quarter 1991 is $505 \mu \mathrm{mhos} / \mathrm{cm}$, which is below the median of $567 \mu \mathrm{mhos} / \mathrm{cm}$, below the interquartile range of 508 to 587 umhos/cm for the well (see Figs. 13 and 14), and among the lowest values obtained for groundwater from well GW-145 (Fig. 14) : $=$ :

GW-142. Downgradient well GW-142 had conductivity values between approximately 300 and $450 \mu$ mhos $/ \mathrm{cm}$ during the base year of CY 1986 (Fig. 15). After the base year, conductivity values for well GW-142 exhibit a generally constant trend with an average of approximately $400 \mu \mathrm{mhos} / \mathrm{cm}$ during the period 1987 through 1989. The conductivity value for first quarter 1991 is $440 \mu \mathrm{mhos} / \mathrm{cm}$, which is above the median of $406 \mu \mathrm{mhos} / \mathrm{cm}$, above the interquartile range of 377 to 435 $\mu \mathrm{mhos} / \mathrm{cm}$ for the well (see Figs. 13 and 15), but within the maximum range of conductivity values observed in the well (see Fig. 15). Throughout the period of observation, the median conductivity values for downgradient well $G W-142$ has been less than that for upgradient well GW-145 (406 $\mu$ mhos/cm compared to 567 $\mu \mathrm{mhos} / \mathrm{cm}$, respectively; see Fig. 13).

\section{Discussion and Summary}

Temporal variations in downgradient wells at the Kerr Hollow Quarry are generally similar to those exhibited by the upgradient well. The temporal $\mathrm{pH}$ trend of the upgradient well is interpreted to represent naturally-occurring, secular $\mathrm{pH}$ fluctuations of the groundwater system surrounding the Kerr Hollow Quarry. All of the downgradient wells exhibit similar trends to that observed in the upgradient well. The temporal pH trend for downgradient wells are essentially identical to that observed in the upgradient well, although the magnitude of the the range of $\mathrm{pH}$ values observed differs from well to well. The similarity of trends suggests that variations in $\mathrm{pH}$ for groundwaters from all wells at the Kerr Hollow Quarry strongly suggests that observed $\mathrm{pH}$ variations are due to secular variations in groundwater chemistry and not to contaminant releases from the site.

The $\mathrm{pH}$ values obtained during the base year of 1986 for the upgradient well and one of the three downgradient wells (GW-142) are always above 7.5, and typically above 8.0. Such values are greater than those typical of uncontaminanted groundwater from the Knox Group studied elsewhere on the Oak Ridge Reservation (HSW 1991a through e). Such values are interpreted to represent partial contamination of the groundwater samples by fluids remaining in the formation from well construction activities. All of the groundwater monitoring wells at the Kerr Hollow Quarry were developed after construction, and purged prior to sampling. However, the fractured 
and karstified nature of the Knox aquifer makes development of wells potentially problematical. Because of such complications, and the fact that $\mathrm{pH}$ values for all wells during subsequent years were within ranges typical of Know Group groundwaters elsewhere in the Oak Ridge area, data obtained during the base period are suspect.

The conductivity values for well GW-142 are less than those of the upgradient well (GW-145) at the site. Additionally, the temporal trends of conductivity values from both well GW-142 and the upgradient well (GW-145) exhibit much variability, with sample to sample changes of $50 \mu \mathrm{mhos} / \mathrm{cm}$. uncommon. Because the temporal conductivity trend in well GW-142 has a similar character to that of the upgradient well, and because conductivity values are less in well GW-142 than those from the upgradient well, the observed conductivity changes are interpreted to be due to secular groundwater variations and not due to a release of contaminants from the site.

Based on the factors discussed above, the statistically significant changes in $\mathrm{pH}$ and conductivity noted for wells at the Kerr Hollow Quarry are interpreted to be false positives, and to not represent a release of contaminants into groundwater from the site.

\section{REFERENCES}

HSW, Inc. 1991a. Groundwater quality assessment for the Bear Creek hydrologic regime at the Y-12 Plant, 1990: Groundwater quality data and calculated rate of contaminant migration. Y/SUB/91-YP507C/1 Part 1.

HSW, Inc. 1991b. Groundwater quality assessment for the Bear Creek hydrologic regime at the Y-12 Plant, 1990: Data interpretation and proposed program modifications. Y/SUB/91-YP507C/1 Part 2.

HSW, Inc. 1991c. Groundwater quality assessment for the Upper East Fork Poplar Creek hydrologic regime at the Y-12 Plant, 1990: Groundwater quality data and calculated rate of contaminant migration. Y/SUB/91-YP507C/2 Part 1.

HSW, Inc. 1991d. Groundwater quality assessment for the Upper East Fork Poplar Creek hydrologic regime at the Y-12 Plant, 1990: Data interpretation and proposed program modifications. Y/SUB/91-YP507C/2 Part 2.

HSW, Inc. 1991e. Groundwater quality assessment for the Chestnut Ridge Security Pits Hazardous Waste Disposal Unit at the Y-12 Plant, 1990. Y/SUB/91YP507C/3. 
ORNL-DH.S OIH-10508

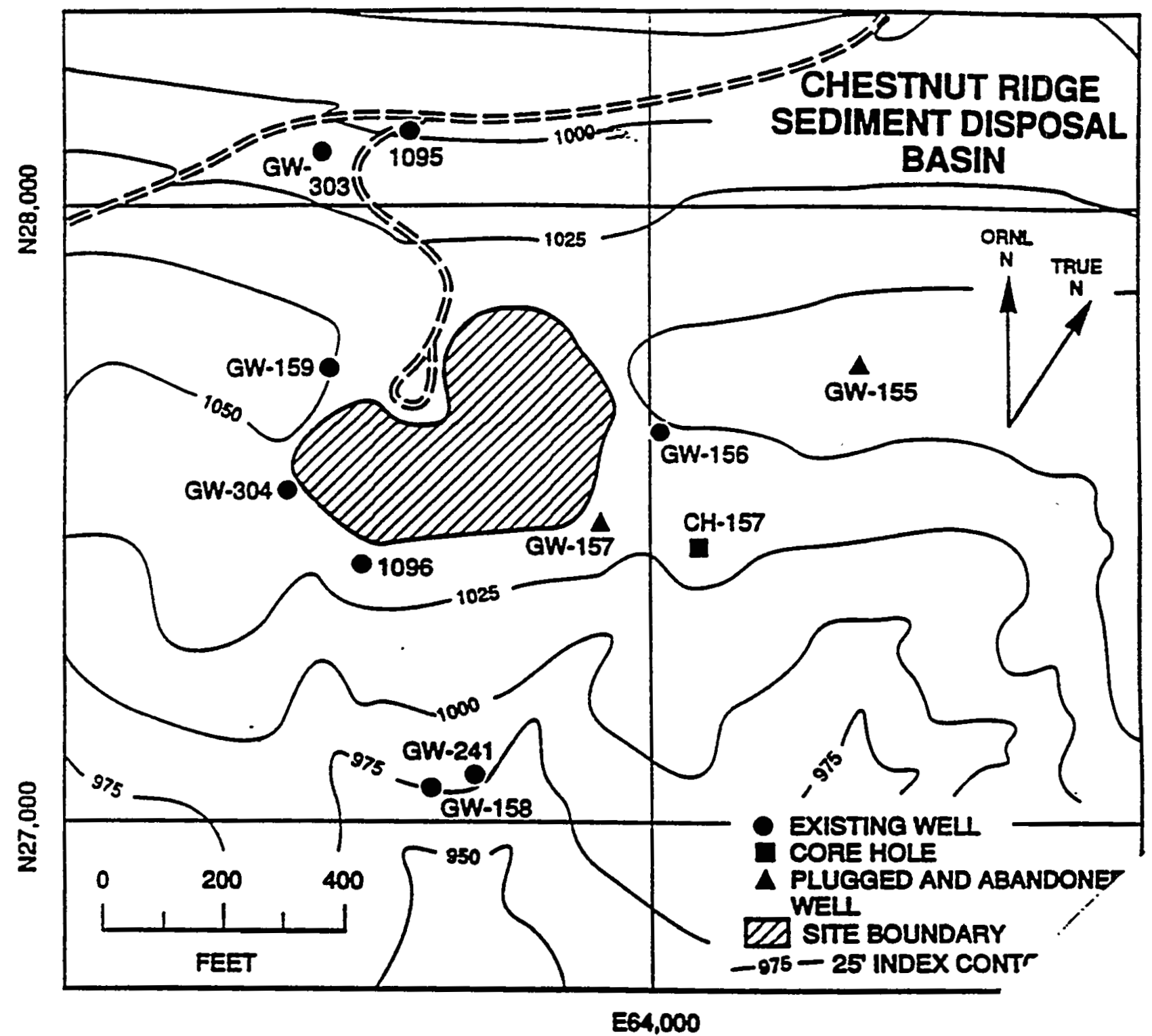

Figure 1. Location map for the Chestnut Ridge Sediment Disposal Basin. Upgradient monitoring well is GW-159. Downgradient monitoring wells are GW-155, GW-156, and GW-157. 


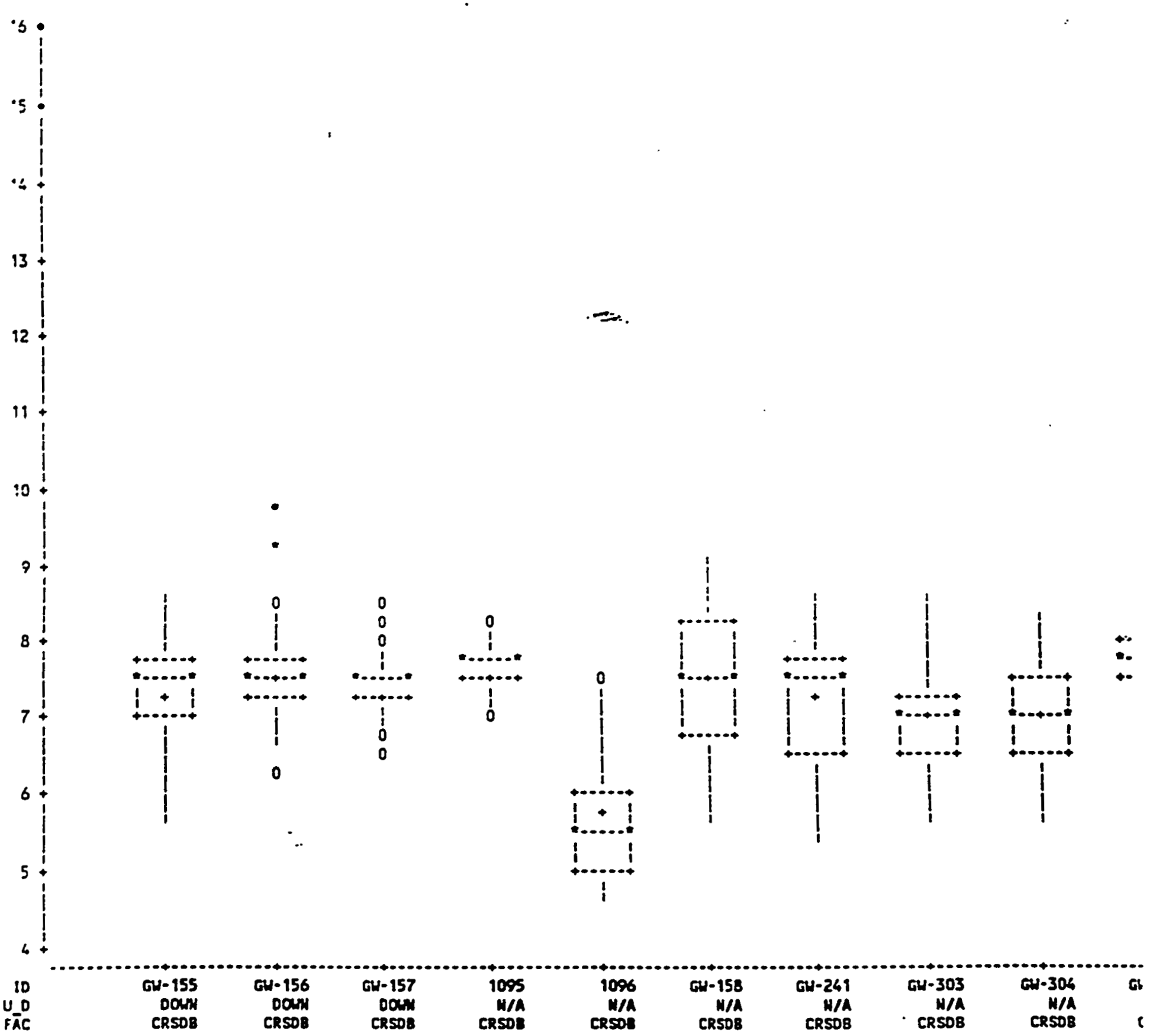

Figure 2. Statistical summary of pH data for groundwater monitoring wells at the Chestnut Ridge Sediment Disposal Basin. Box outlines represent the 25th percentile (bottom of box), median (middle line terminated with asterisks), and 75th percentile (top of box) of the data set for a individual well. Mean is indicated by central plus sign $(t)$ within the box. Central lines or tails extending above and below the box indicate the range of outliers within 1.5 interquartile ranges below the 25th percentile and above the 75th percentile. Outliers beyond this range are illustrated by zero $(0)$ if they are within 3 interquartile ranges. More extreme outliers are illustrated by an asterisk (*). Because of scale, some boxes appear incomplete. 
$-\frac{\overline{2}}{2}$

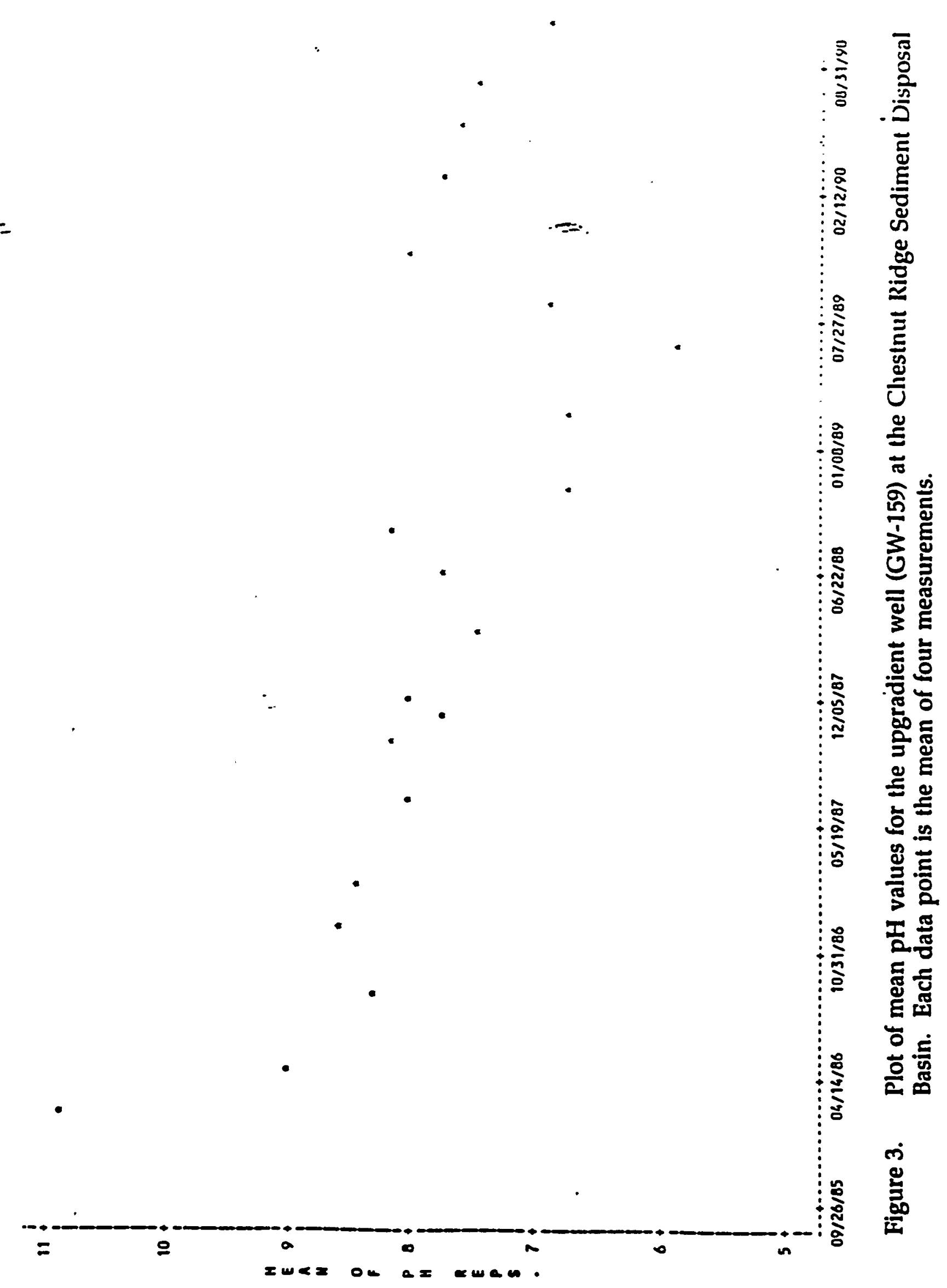




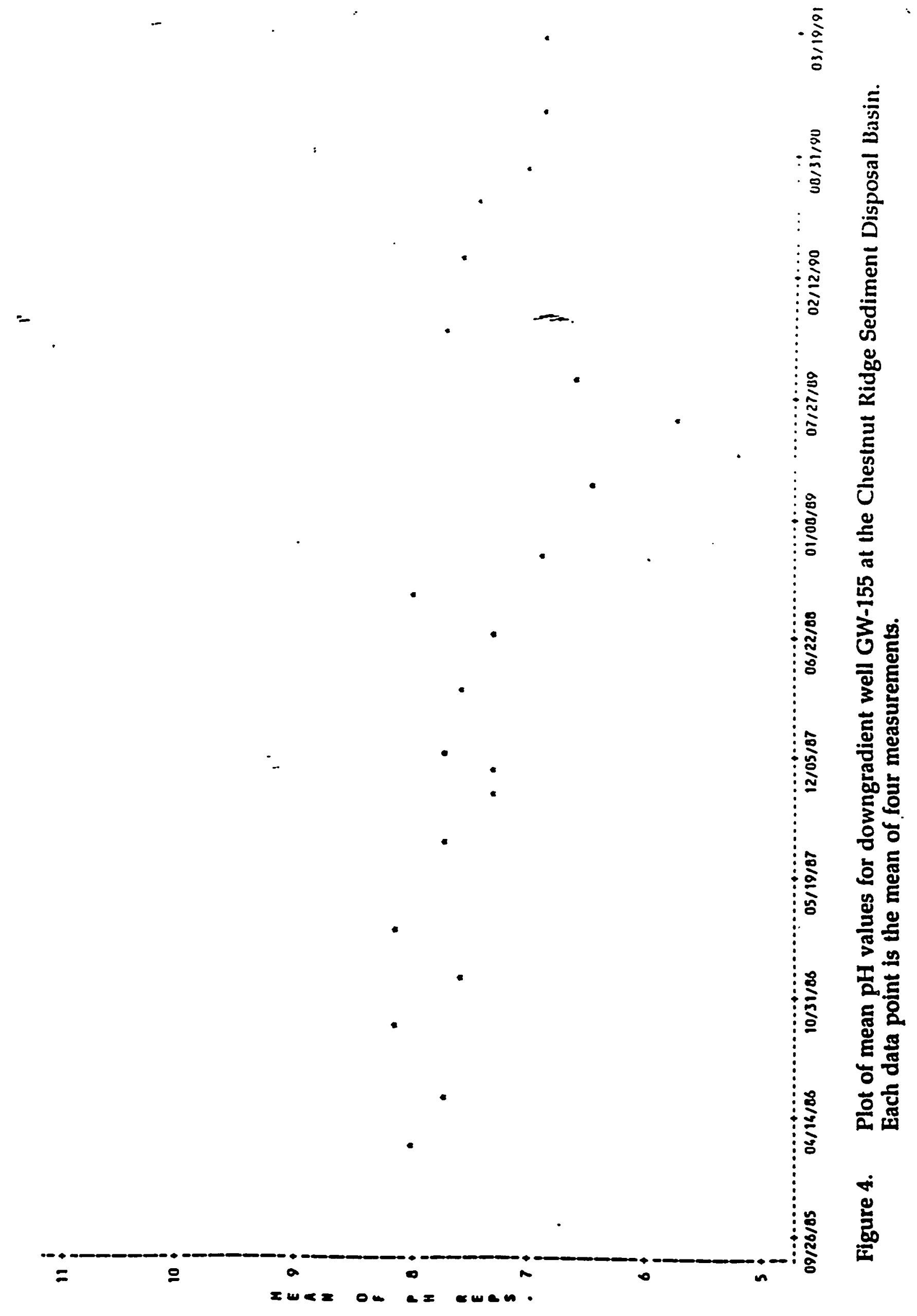




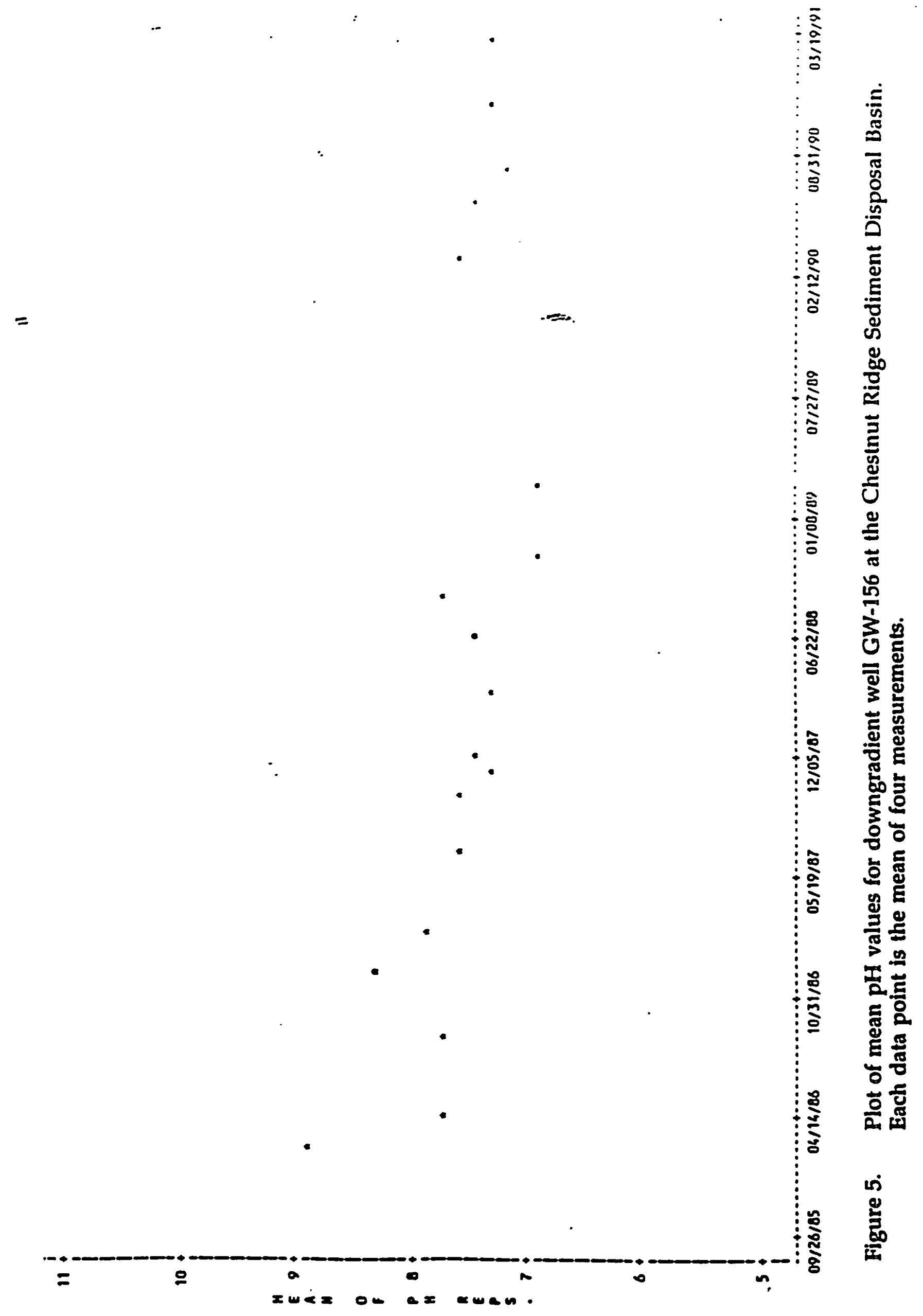


ORNL-DAN 91M-105

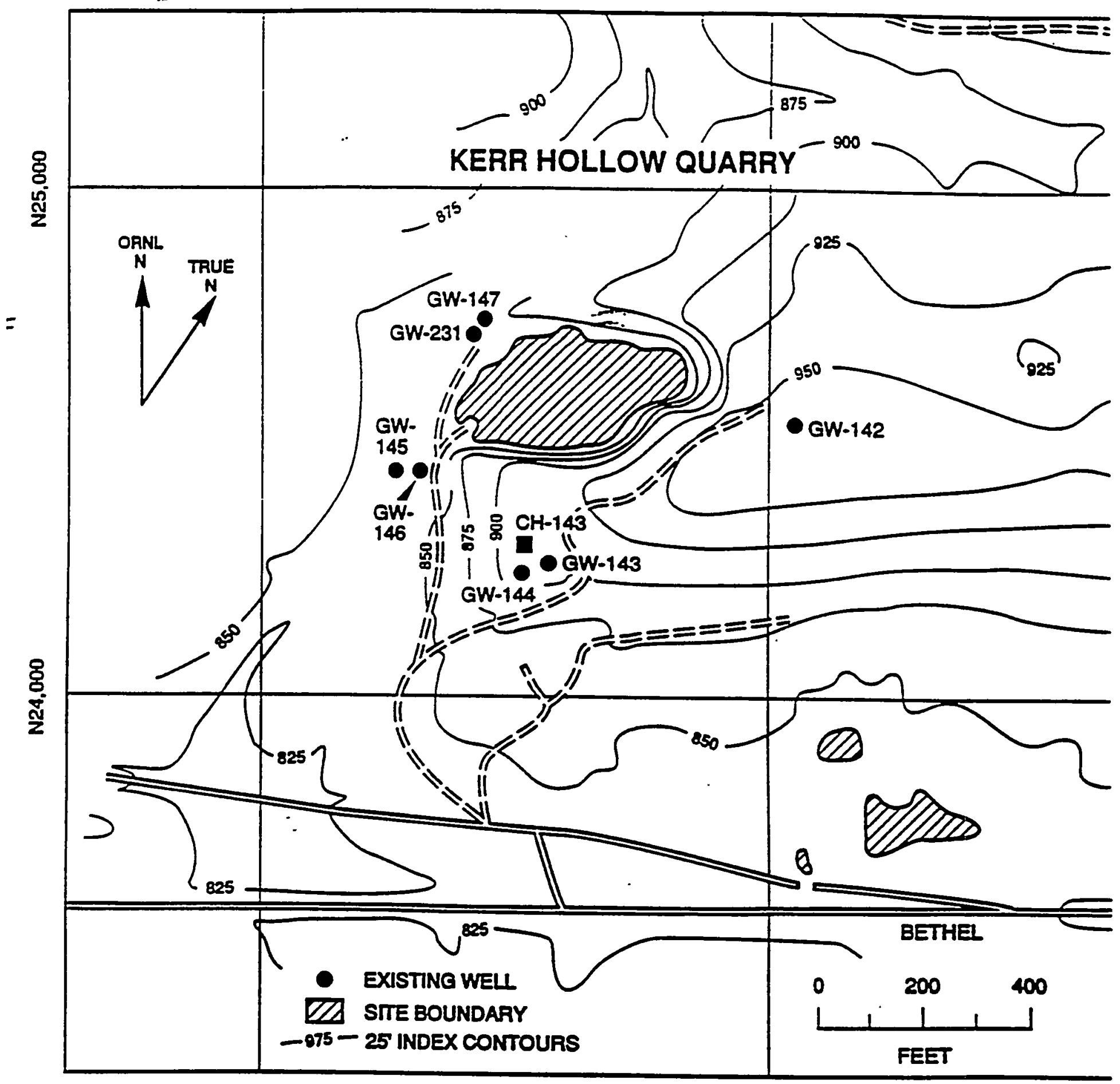

$E 63,000$

$E 64,000$

Figure 7. Location map for the Kerr Hollow Quarry. Upgradient monitoring well is GW-145. Downgradient monitoring wells are GW-142, GW-144, and GW-231. 


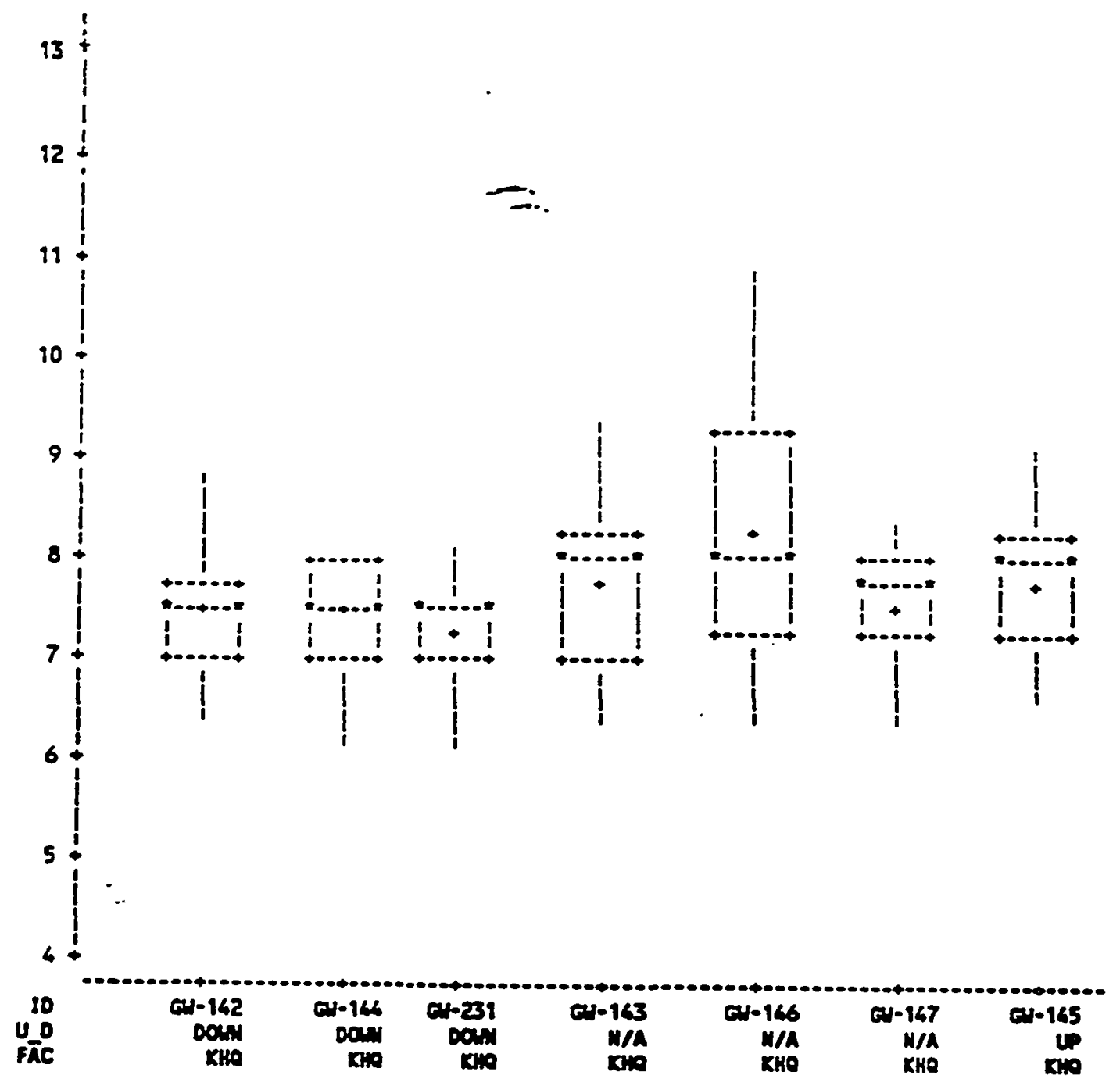

Figure 8. Statistical summary of pH data for groundwater monitoring wells at the Kerr Hollow Quarry. Box outlines represent the 25th percentile (bottom of box), median (middle line terminated with asterisks), and 75th percentile (top of box) of the data set for a individual well. Mean is indicated by central plus sign ( $t$ ) within the box. Central lines or tails extending above and below the box indicate the range of outliers within 1.5 interquartile ranges below the 25th percentile and above the 75th percentile. Outliers beyond this range are illustrated by zero $(0)$ if they are within 3 interquartile ranges. More extreme outliers are illustrated by an asterisk (*). Because of scale, some boxes appear incomplete. 


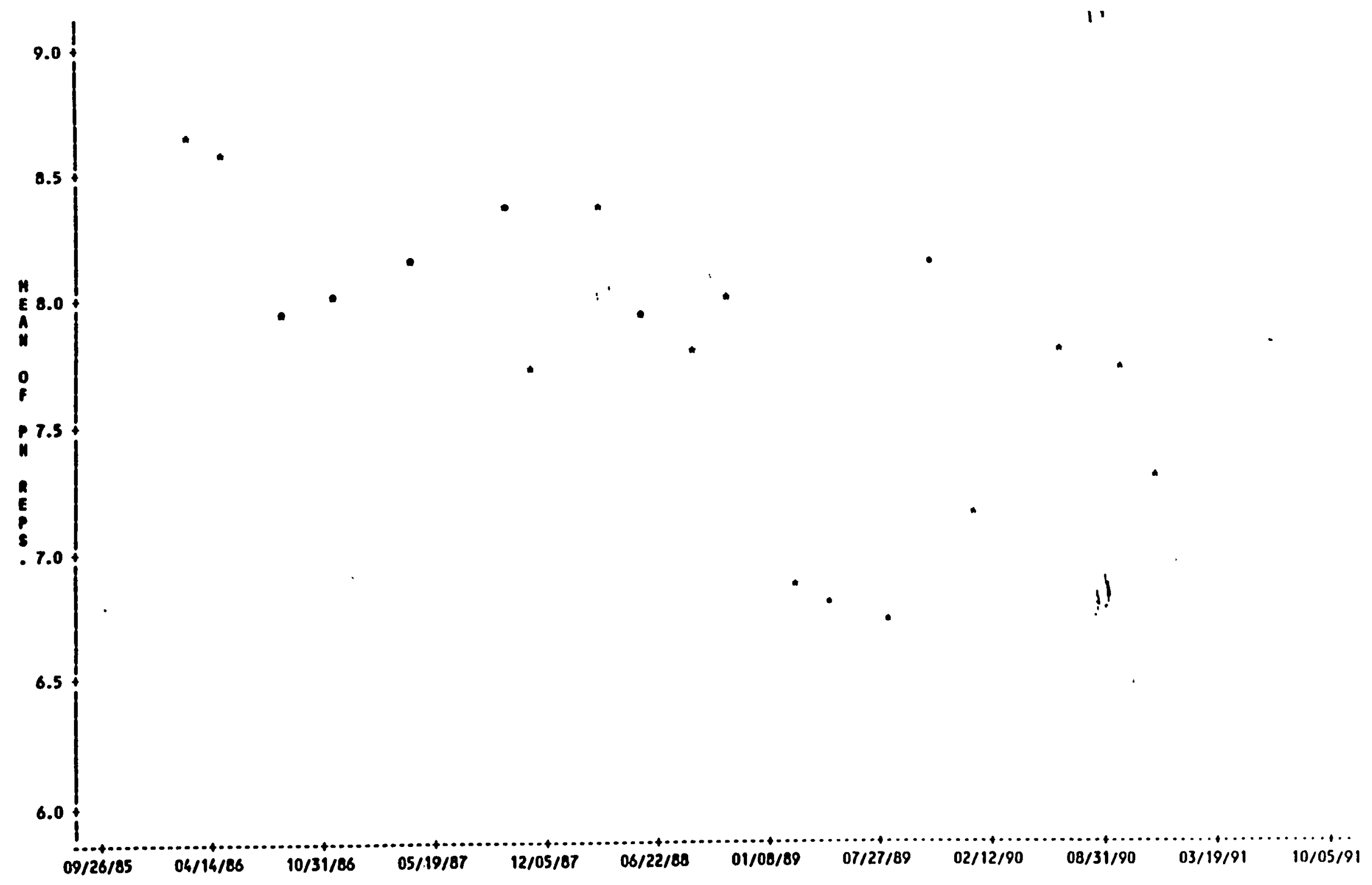

Figure 9. Plot of mean pH values for upgradient well GW-145 at Kerr Hollow Quarry. Each data point is the mean of four measurements. 


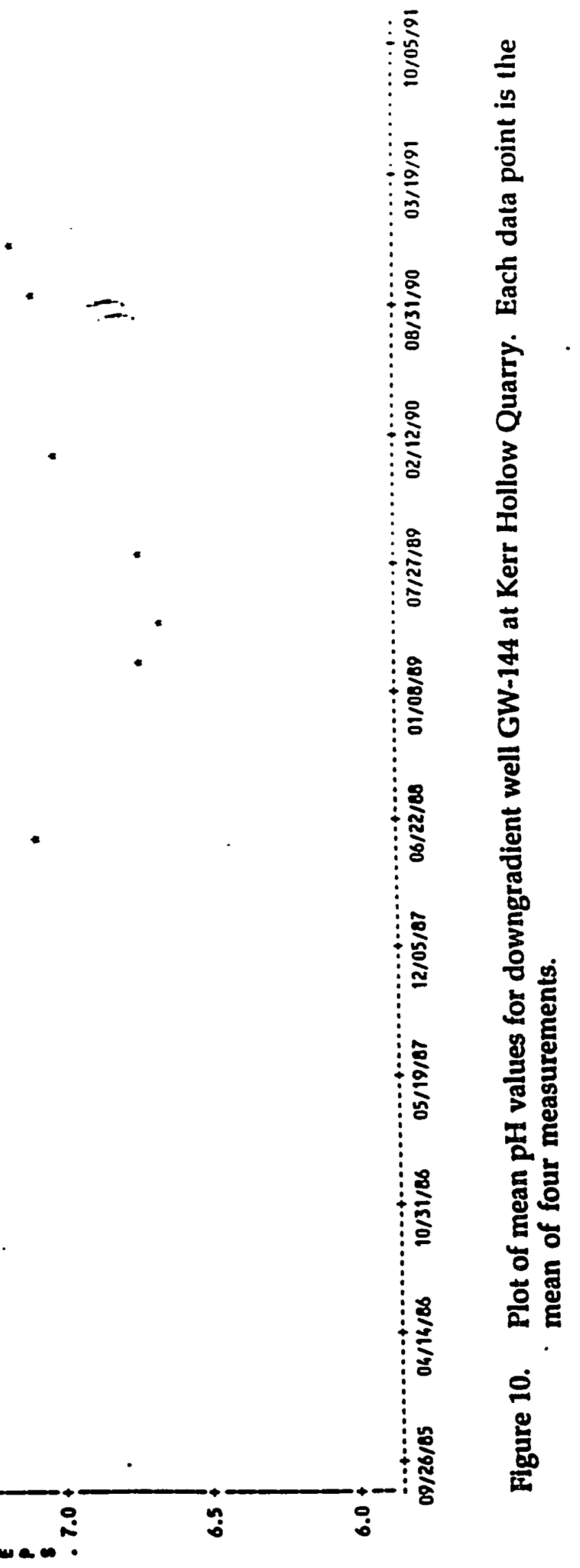




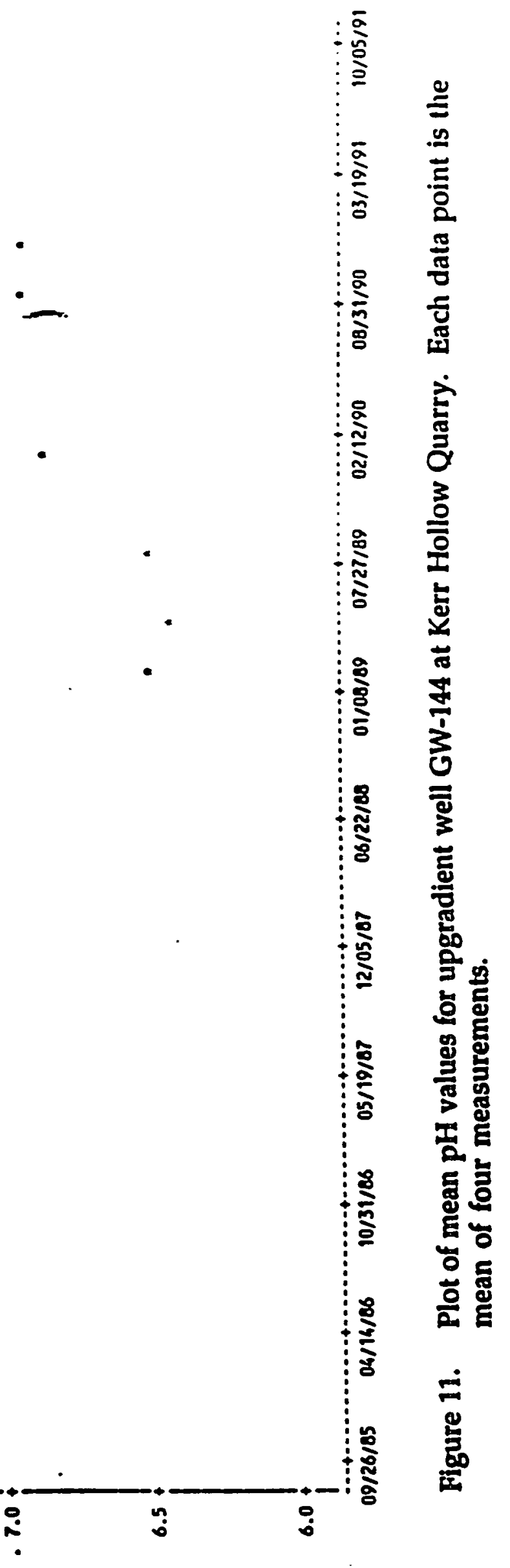




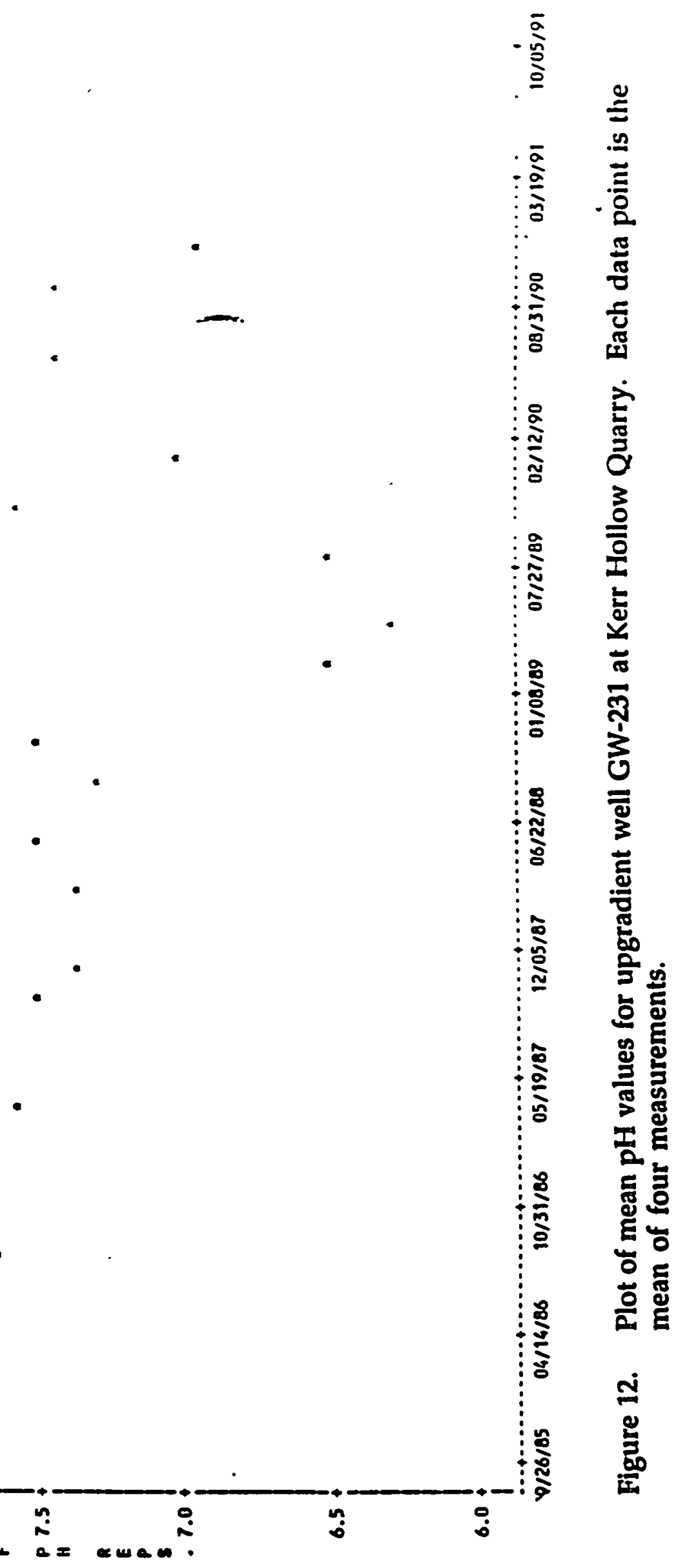




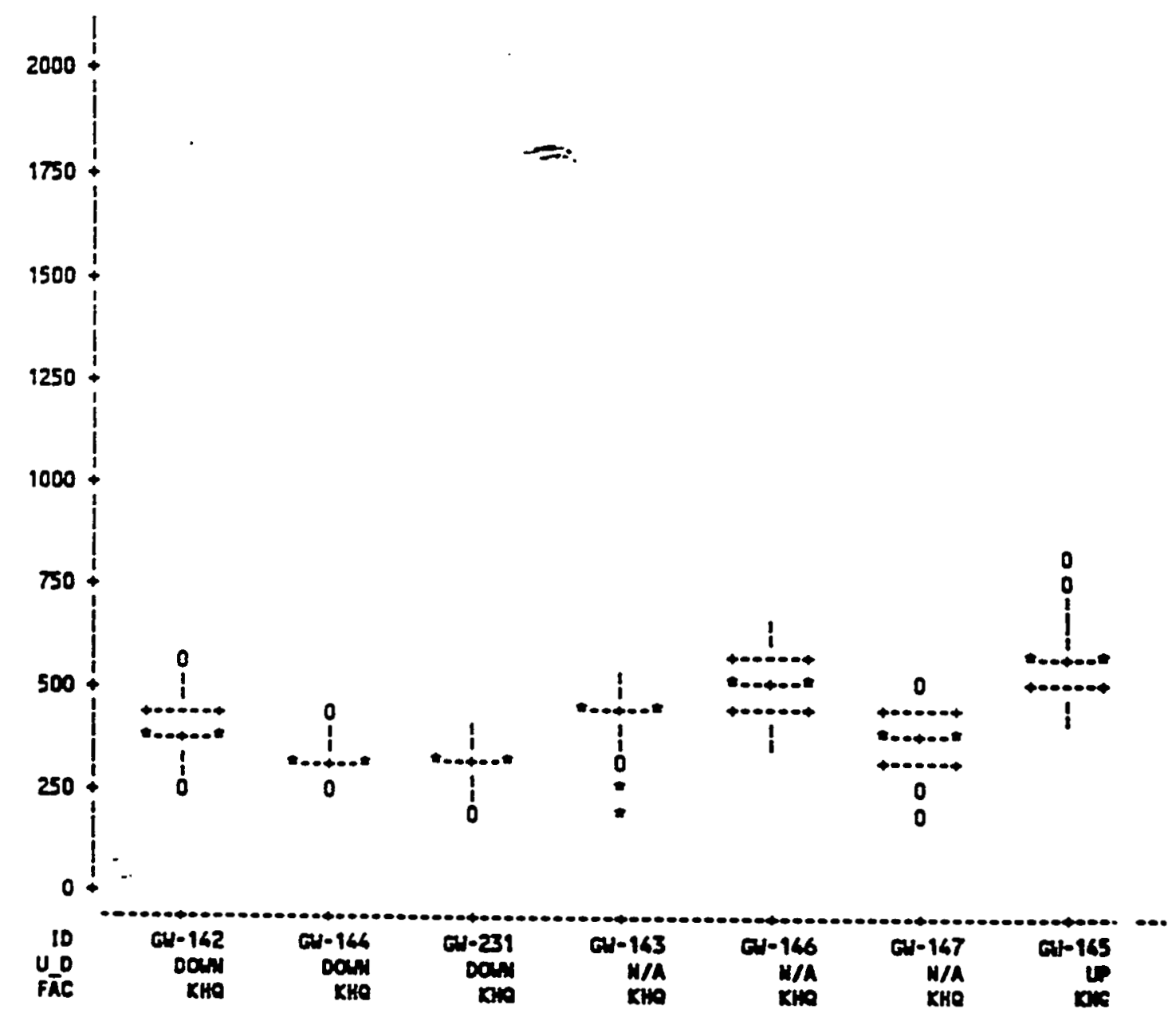

Figure 13. Statistical summary of conductivity data for groundwater monitc ing wells at the Kerr Hollow Quarry. Box outlines represent the 25th percentile (bottom of box), median (middle line terminated with asterisks), and 75th percentile (top of box) of the data set or a individual well. Mean is indicated by central plus sign ( + ) withi 1 the box. Central lines or tails extending above and below the box in icate the range of outliers within 1.5 interquartile ranges below the 25th percentile and above the 75th percentile. Outliers beyond this ran re are illustrated by zero (0) if they are within 3 interquartile ranges. More extreme outliers are illustrated by an asterisk $\left(^{*}\right)$. Because of scale, some boxes appear'incomplete. 


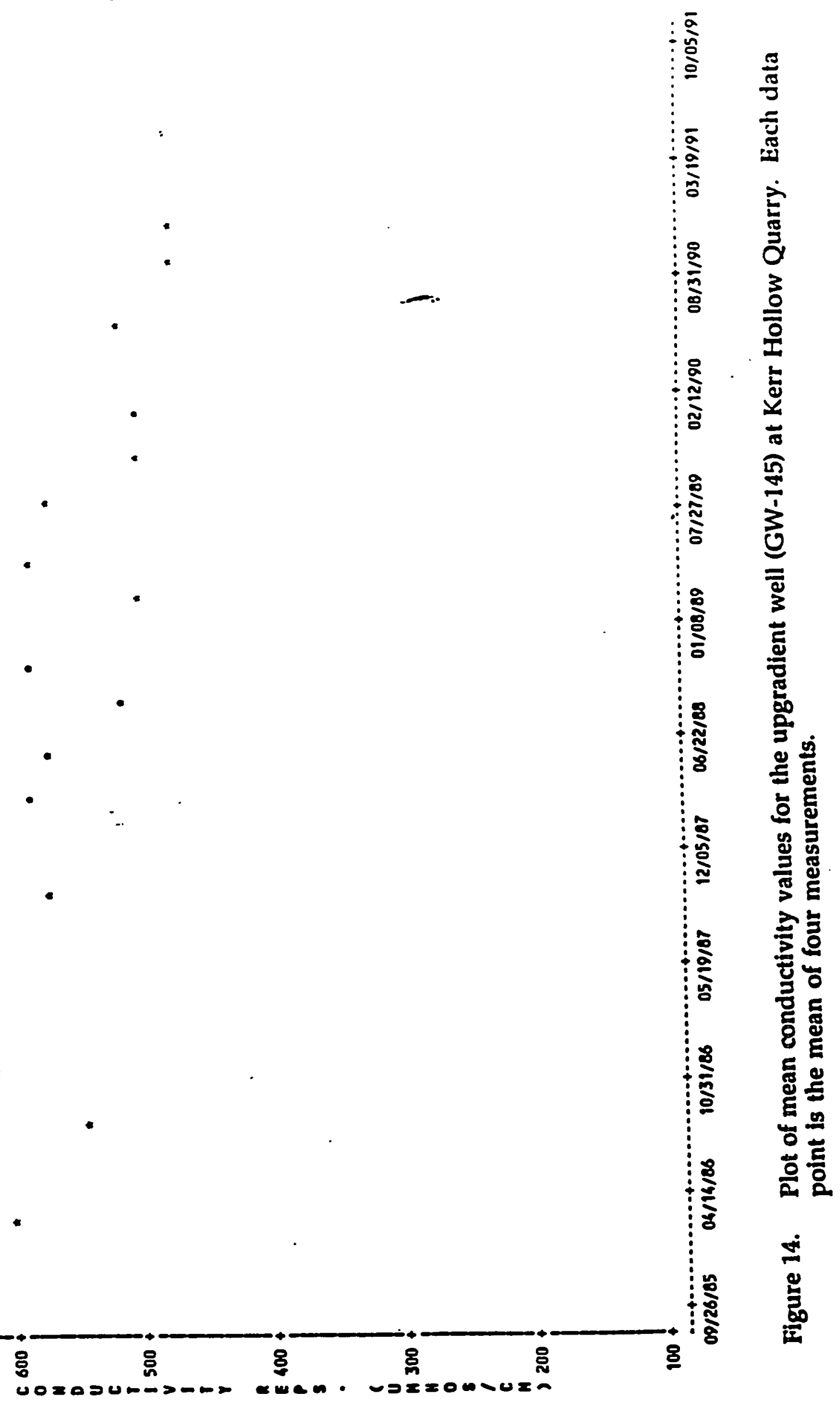





\title{
EVALUATION OF TOTAL ORGANIC CARBON AND TOTAL ORGANIC HALIDE MEASUREMENTS FOR GROUNDWATER FROM THE CHESTNUT RIDGE SEDIMENT DISPOSAL BASIN AND KERR HOLLOW QUARRY
}

\section{OAK RIDGE Y-12.PLANT}

\author{
C. Stephen Haase ${ }^{3}$ \\ Helen L. King 2 \\ Terre M. Mercier 3
}

- November 25, 1991

1 Environmental Sciences Division, Oak Ridge National Laboratory, P. O. Box 2008, Oak Ridge, Tennessee 37831-6352

2 Environmental Management Department, Oak Ridge Y-12 Plant, P. O. Box 2009, Oak Ridge, Tennessee 37831-8219

3 H \& R Technical Associates, Oak Ridge, Tennessee 37830 


\section{Introduction}

Groundwater quality data were collect for two sites in RCRA Interim Status Detection Monitoring at the Oak Ridge Y-12 Plant during the first quarter of calendar vear (CY) 1991. As required by Rule 1200-1-1-.05(6) of the Tennessee Department of Environment and Conservation, monitoring results for indicator parameters ( $\mathrm{pH}$, conductivity, total organic carbon (TOC), and total organic halides (TOX)) were reviewed to determine if a statistically significant change had occurred in the values of one or more indicator parameters. Analysis of the data obtained during the period 1.987 through 1990 indicated that statistically significant changes had sporadically occurred in both TOC and TOX for groundwater samples obtained from upgradient and downgradient wells at both the Chestnut Ridge Sediment Disposal Basin and Kerr Hollow Quarry.

The purpose of this document is to review TOC and TOX data for groundwater monitoring wells at both facilities to determine if the statistically significant changes in the indicator parameters noted represent a release of contaminants into the groundwater from the facilities, or if the changes observed are due to other, naturally occurring factors.

\section{Chestnut Ridge Sediment Disposal Basin}

Evaluation of TOC Data

Total organic carbon measurements were obtained from groundwater monitoring wells at the Chestnut Ridge Sediment Disposal Basin (Fig. 1) beginning in CY 1987. The mean, median, and variance of TOC measurements are illustrated in Fig. 2. The data indicate that (1) in wells GW-155, GW-158, GW-241, GW-159, and No. 1096 the majority of TOC values obtained were below the detection limit of $1 \mathrm{mg} / \mathrm{L}$, (2) for wells GW-156, GW-157, GW-303, GW-304, and No. 1095 the majority of TOC values are above the detection limit, and for these wells the median TOC values are less than $5 \mathrm{mg} / \mathrm{L}$, (3) for wells GW-156, GW-157, GW-303, GW-304, and No. 1095 the degree of variance, as calculated by the distance between the 25th and 75th percentiles, is typically less than $1 \mathrm{mg} / \mathrm{L}$, and (4) the greatest $T O C$ values measured in any well during the period of observation was $7.1 \mathrm{mg} / \mathrm{L}$ from well GW-157.

Total organic carbon measurements reflect dissolved and suspended organic carbon within a groundwater sample. Naturally occurring humic and fulvic acids are major contributors to the dissolved component, and suspended organic material and soil debris are major contributors to the suspended component. In contaminated groundwaters, dissolved organic chemicals, such as petroleum products, chlorinated solvents, and chemically complex organic leachates are typically major contributors to TOC values. 
Trend plots for TOC measurements from the upgradient and downgradient wells at the Chestnut Ridge Sediment Disposal Basin are illustrated in Figs. 3a through 6a. To evaluate whether the fluctuations observed in TOC values are naturallyoccurring or are due to influxes of contaminant-related organic carbon, trend plots for summed chlorinated and aromatic volatile organic compounds (VOCs) are illustrated in Figs. $3 \mathrm{~b}$ through $6 \mathrm{~b}$. Volatile organic compounds, such as carbon tetrachloride and trichloroethene, are the major potential organic contaminants at the Chestnut Ridge Sediment Disposal Basin and, therefore, are the only contaminant-related source of organic carbon that could impact TOC values in groundwater. Data for TOC and summed chlorinated and aromatic VOC measurements for the upgradient and downgradient monitoring wells is summarized and discussed in the following sections.

$G W-159$. The majority of TOC values for upgradient well GW-159 were below the detection limit of $1 \mathrm{mg} / \mathrm{L}$ (Fig. 3a). Three samples obtained during the period 1989 through 1990 were above detection limits and ranged between 2 and $3 \mathrm{mg} / \mathrm{L}$. Summed chlorinated and aromatic VOCs for well GW-159 range between 0 and $10 \mathrm{\mu g} / \mathrm{L}$ (see Fig. 3b: note these concentrations are a factor of 1000 less than those of TOC values). None of the three TOC values above detection limits corresponds to elevated summed chlorinated and aromatic VOCs values, suggesting that the variations in TOC values noted are due to natural, secular fluctuations in the upgradient well.

GW-155. The majority of TOC values for downgradient well GW-155 were below the detection limit of $1 \mathrm{mg} / \mathrm{L}$ (Fig. 4a). Four samples obtained during the period 1989 through 1990 were above detection limits and ranged between 2 and $3 \mathrm{mg} / \mathrm{L}$, which is within the range of TOC values noted for the upgradient well. Summed chlorinated and aromatic VOCs for well GW-155 range between 0 and $5 \mu \mathrm{g} / \mathrm{L}$ (see Fig. 4b: note these concentrations are a factor of 1000 less than those of TOC values). Only one of the four TOC values above detection limits corresponds to a nonzero summed chlorinated and aromatic VOCs value, but the smallness of the summed chlorinated and aromatic VOCs value $(5 \mu \mathrm{g} / \mathrm{L})$ compared to the TOC value $(\sim 1.5 \mathrm{mg} / \mathrm{L})$ suggests that the TOC value represents natural-occurring fluctuations.

GW-156. The majority of TOC values for downgradient well GW-156 were above the detection limit of $1 \mathrm{mg} / \mathrm{L}$ (Fig. 5a). Samples obtained during the period 1987 through 1990 that were above the detection limit had a median of $2 \mathrm{mg} / \mathrm{L}$ and ranged from the detection limit of 1 to $-4.5 \mathrm{mg} / \mathrm{L}$. This range of TOC values is greater than that noted for those samples from the upgradient well, but the median value of $2 \mathrm{mg} / \mathrm{L}$ is within the range noted for the upgradient well. Summed chlorinated and aromatic VOCs for well GW-156 range between 0 and $-12 \mu \mathrm{g} / \mathrm{L}$ (see Fig. 5b: note these concentrations are a factor of 1000 less than those of TOC values). During the period 1988 through 1990, however all summed chlorinated and aromatic VOCs values were 0 . The fluctuations observed in TOC during that period, therefore, cannot be due to the introduction of contaminant-related organic carbon and must represent natural-occurring fluctuations. 
$G W-157$. All TOC values for downgradient well $G W-157$ were above the $c$ stection limit of $1 \mathrm{mg} / \mathrm{L}$ (Fig. 6a). Samples obtained during the period 1987 through $\cdot 90 \mathrm{had}$ a median of $3 \mathrm{mg} / \mathrm{L}$ and ranged from the detection limit of 1 to $-5.5 \mathrm{mg}$, . This range of TOC values is greater than that noted for those samples $i$ om the upgradient well, but the median value of $2 \mathrm{mg} / \mathrm{L}$ is within the range notc.. . J the upgradient well. Summed chlorinated and aromatic VOCs for well GW-i : 5 range between 0 and $\sim 45 \mu \mathrm{g} / \mathrm{L}$ (see Fig. $6 \mathrm{~b}$ : note these concentrations are a factor of 1000 less than those of TOC values). Only three summed chlorinated and arom ric VOC values were above $10 \mu \mathrm{g} / \mathrm{L}$, however, and the majority of values is within he range 0 to $5 \mu \mathrm{g} / \mathrm{L}$. Examination of Figs. 6a and 6b indicates that there is no $\mathrm{c}$ rrelation between increases in TOC values and increases in summed chlorin ted and aromatic VOCs values, suggesting that the TOC fluctuations represen naturaloccurring variations.

Discussion and Summary. Temporal variations in TOC values for both the upgradient and downgradient wells at the Chestnut Ridge Sediment Disf Jsal Basin exhibit no identifiable trend or relationship to each other. Many TOC va ses for all wells are below the $1 \mathrm{mg} / \mathrm{L}$ detection limit, and values above the dete tion limit typically range between 2 and $3 \mathrm{mg} / \mathrm{L}$. Such a range is within what is e: pected for uncontaminated groundwaters (Hem 1986; Drever 1988). It is also tyf cal of the range noted for uncontaminated groundwaters that occur elsewhere $c a$ the Oak Ridge Reservation (HSW 1991 a-e). Variations in TOC for a well are no correlated with variations in the summed chlorinated and aromatic VOC values $i$ that well. In all case variations in summed chlorinated and aromatic VOC value are small, with the values at or near $0 \mu \mathrm{g} / \mathrm{L}$.

Based on the factors discussed above, the statistically significant char jes in TOC noted for wells at the Chestnut Ridge Sediment Disposal Basin are inter rreted to be false positives, and to not represent a release of contaminants into roundwater from the site.

\section{Evaluation of TOX Data}

Total organic halide measurements were obtained from groundwate 'monitoring wells at the Chestnut Ridge Sediment Disposal Basin (Fig. 1) beginnin ; in CY 1986. The mean, median, and variance of TOX measurements are illustra ed in Fig. 7. The data indicate that (1) much of the data for all wells is less thar the $10 \mu \mathrm{g} / \mathrm{L}$ detection limit, and because of this the median values for all we ls are at the detection limit, (2) six of the ten wells at the site have extreme or tliers, (3) the extreme outliers for the upgradient well (GW-159) range up to $-900 \mathrm{dg} / \mathrm{L}$, and (4) extreme outliers for the downgradient wells range up to $\sim 400 \mu \mathrm{g} / \mathrm{L}(\mathrm{G} V-156)$.

Trend plots for TOX measurements from the upgradient and downgr adient wells at the Chestnut Ridge Sediment Disposal Basin are illustrated in Figs. $\{$ a through 11a. 
For comparison, trend plots of summed chlorinated VOCs are presented in Figs. $8 \mathrm{~b}$ through 11b. Data for TOX and summed chlorinated VOC measurements for the upgradient and downgradient monitoring wells is summarized and discussed in the following sections.

GW-159. The majority of TOX values for upgradient well GW-159 were less than 25 $\mathrm{Hg} / \mathrm{L}$ (Fig. 8a). Samples obtained during CY 1986 range from the detection limit of 10 $\mu \mathrm{g} / \mathrm{L}$ to $-900 \mu \mathrm{g} / \mathrm{L}$. Subsequent to 1986 no TOX values greater than $25 \mu \mathrm{g} / \mathrm{L}$ were obtained, which suggests that the results for CY 1986 represent spurious analytical data. Summed chlorinated VOCs for well GW-159 during the period 1986 through 1990 range between 0 and $8 \mu \mathrm{g} / \mathrm{L}$ (Fig. $8 \mathrm{~b}$ ), with the results for the period 1987 through 1990 all being less than $5 \mu \mathrm{g} / \mathrm{L}$. No consistent correlation or temporal pattern in TOX and summed chlorinated VOC values is noted, suggesting that the variability observed likely represents analytical uncertainties.

GW-155. With one exception, all TOX values for downgradient well GW-155 were less than $50 \mu \mathrm{g} / \mathrm{L}$ (Fig. 9a), and the majority of values are below the detection limit of $10 \mu \mathrm{g} / \mathrm{L}$. Samples obtained during CY 1986 range from the detection limit of 10 $\mu \mathrm{g} / \mathrm{L}$ to $\sim 90 \mu \mathrm{g} / \mathrm{L}$. Subsequent to 1986 no TOX values greater than $30 \mu \mathrm{g} / \mathrm{L}$ were obtained, which suggests that the results for CY 1986 represent spurious analytical data. Summed chlorinated VOCs for well GW-155 during the period 1986 through 1990 range between 0 and $5 \mu \mathrm{g} / \mathrm{L}$ (Fig. 9b). No consistent correlation or temporal pattern in TOX and summed chlorinated VOC values is noted, suggesting that the variability observed likely represents analytical uncertainties.

GW-156. With two exceptions, all TOX values for downgradient well GW-156 were less than $\sim 30 \mu \mathrm{g} / \mathrm{L}$ (Fig. 10a), and the majority of values are below the detection limit of $10 \mu \mathrm{g} / \mathrm{L}$. Samples obtained during CY 1986 range from the detection limit of 10 $\mu \mathrm{g} / \mathrm{L}$ to $\sim 350 \mu \mathrm{g} / \mathrm{L}$. Subsequent to 1986 no TOX values greater than $30 \mu \mathrm{g} / \mathrm{L}$ were obtained, which suggests that the results for CY 1986 represent spurious analytical data. Summed chlorinated VOCs for well GW-156 during the period 1987 through 1990 range from 0 to $-8 \mu \mathrm{g} / \mathrm{L}$ (Fig. 10b), with the results for the period 1988 through 1990 all being $0 \mu \mathrm{g} / \mathrm{L}$. No consistent correlation or temporal pattern in TOX and summed chlorinated VOC values is noted, suggesting that the variability observed likely represents analytical uncertainties.

GW-157. With one exception, all TOX values for downgradient well GW-157 were less than $-50 \mu \mathrm{g} / \mathrm{L}$ (Fig. 11a), and the majority of values are below the detection limit of $10 \mu \mathrm{g} / \mathrm{L}$. Samples obtained during CY 1986 range from the detection limit of 10 $\mu \mathrm{g} / \mathrm{L}$ to $-250 \mu \mathrm{g} / \mathrm{L}$. Subsequent to 1986 no TOX values greater than $50 \mu \mathrm{g} / \mathrm{L}$ were obtained, which suggests that the results for CY 1986 represent spurious analytical data. During the period 1989 through 1990, TOX values were less than $-20 \mu \mathrm{g} / \mathrm{L}$. Summed chlorinated VOCs for well GW-157 during the period 1987 through 1990 range from 0 to $-45 \mu \mathrm{g} / \mathrm{L}$ (Fig. 11b). With one exception, the results for the period 1988 through 1990 were all between 0 and $5 \mu \mathrm{g} / \mathrm{L}$. No consistent correlation or 
temporal pattem in TOX and summed chlorinated VOC values is noted, suggesting that the variability observed likely represents analytical uncertainties.

Discussion and Summary. Temporal variations in TOX values for both the upgradient and downgradient wells at the Chestnut Ridge Sediment Disposal Basin exhibit no identifiable trend or relationship to each other. Many TOX values for all wells are below the $10 \mu \mathrm{g} / \mathrm{L}$ detection limit, and values above the detection limit typically range between 10 and $50 \mu \mathrm{g} / \mathrm{L}$. Variations in TOX for a well are not correlated with variations in the summed chlorinated and aromatic VOC values in that well. In all cases variations in summed chlorinated and aromatic VOC values are small, with the values at or near $0 \mu \mathrm{g} / \mathrm{L}$.

Based on the factors discussed above, the statistically significant changes in TOX noted for wells at the Chestnut Ridge Sediment Disposal Basin are interpreted to be false positives, and to not represent a release of contaminants into groundwater from the site.

\section{Kerr Hollow Quarry}

\section{Evaluation of TOC Data}

Total organic carbon measurements were obtained from groundwater monitoring wells at Kerr Hollow Quarry (Fig. 12) beginning in CY 1987. The mean, median, and variance of TOC measurements are illustrated in Fig. 13. The data indicate that (1) in all wells the majority of TOC values obtained were below the detection limit of 1 $\mathrm{mg} / \mathrm{L},(2)$ although the median value for TOC in all wells is at or close to the detection limit of $1 \mathrm{mg} / \mathrm{L}$, most wells have several outliers whose values can be as large as $-60 \mathrm{mg} / \mathrm{L}$, and (3) because many of the data are less than the detection limit, the TOC data set is truncated, making statistical analysis difficult.

Total organic carbon measurements reflect dissolved and suspended organic carbon within a groundwater sample. Naturally occurring humic and fulvic acids are major contributors to the dissolved component, and suspended organic material and soil debris are major contributors to the suspended component. In contaminated groundwaters, dissolved organic chemicals, such as petroleum products, chlorinated solvents, and chemically complex organic leachates are typically major contributors to TOC values.

Trend plots for TOC measurements from the upgradient and downgradient wells at Kerr Hollow Quarry are illustrated in Figs. 14a through 17a. To evaluate whether the fluctuations observed in TOC values are naturally-occurring or are due to influxes of contaminant-related organic carbon, trend plots for summed chlorinated and aromatic volatile organic compounds (VOCs) are illustrated in Figs. 14b through 17b. Volatile organic compounds, such as carbon tetrachloride and trichloroethene, are the major potential organic contaminants at.Kerr Hollow Quarry and, therefore, 
are the only contaminant-related source of organic carbon that could impact TOC values in groundwater. Data for TOC and summed chlorinated and aromatic VOC measurements for the upgradient and downgradient monitoring wells is summarized and discussed in the following sections.

$G W-145$. The TOC values for upgradient well $G W-145$ range from the derection limit of $1 \mathrm{mg} / \mathrm{L}$ to $\sim 3.3 \mathrm{mg} / \mathrm{L}$ (Fig. 14a), and, with the exception of one sample, all TOC values are less than $2 \mathrm{mg} / \mathrm{L}$ : Summed chlorinated and aromatic VOCs for well GW-145 are $0 \mu \mathrm{g} / \mathrm{L}$ throughout the period of interest (see Fig. 14b: note these concentrations are a factor of 1000 less than those of TOC values). These data suggest that the variations in TOC values noted are due to natural, secular fluctuations in the upgradient well, and not to contaminant-related organic carbon sources.

$G W-142$. The TOC values for downgradient well GW-142 range from the detection limit of $1 \mathrm{mg} / \mathrm{L}$ to $\sim 6.3 \mathrm{mg} / \mathrm{L}$ (Fig. 15a). With the exception of two samples, all TOC values are less than $2 \mathrm{mg} / \mathrm{L}$, which is within the range of values noted for the upgradient well. With one exception, summed chlorinated and aromatic VOCs for well GW-142 range from 0 to $\sim 3 \mu \mathrm{g} / \mathrm{L}$ throughout the period of interest, with most values being $0 \mathrm{mg} / \mathrm{L}$ (see Fig. 15b: note these concentrations are a factor of 1000 less than those of TOC values). There is no correlation between IOC and summed chlorinated and aromatic VOCs variations, which suggests that the variations in TOC values noted are due to natural, secular fluctuations, and not to contaminantrelated organic carbon sources.

GW-144. The TOC values for downgradient well GW-144 range from the detection limit of $1 \mathrm{mg} / \mathrm{L}$ to $\sim 4 \mathrm{mg} / \mathrm{L}$ (Fig. 16a). With the exception of one sample, all TOC values are less than $-2 \mathrm{mg} / L$, which is within the range of values noted for the upgradient well. Summed chlorinated and aromatic VOCs for well GW-144 range from 0 to $\sim 4 \mu \mathrm{g} / \mathrm{L}$ throughout the period of interest, with most values being $0 \mathrm{mg} / \mathrm{L}$ (see Fig. 16b: note these concentrations are a factor of 1000 less than those of TOC values). There is no correlation between TOC and summed chlorinated and aromatic VOCs variations, which suggests that the variations in TOC values noted are due to natural, secular fluctuations, and not to contaminant-related organic carbon sources.

GW-231. The TOC values for downgradient well GW-231 range from the detection limit of $1 \mathrm{mg} / \mathrm{L}$ to $\sim 5.7 \mathrm{mg} / \mathrm{L}$ (Fig. 17a). With the exception of two samples, all TOC values are less than $2 \mathrm{mg} / \mathrm{L}$, which is within the range of values noted for the upgradient well. Summed chlorinated and aromatic VOCs for well GW-23I range from 0 to $-3 \mu \mathrm{g} / \mathrm{L}$ throughout the period of interest, with most values being $0 \mathrm{mg} / \mathrm{L}$ (see Fig. 17b: note these concentrations are a factor of 1000 less than those of TOC values). There is no correlation between TOC and summed chlorinated and aromatic VOCs variations, which suggests that the variations in TOC values noted are due to natural, secular fluctuations, and not to contaminant-related organic carbon sources. 
Discussion and Summary. Temporal variations in TOC values for both the upgradient and downgradient wells at the Kerr Hollow Quarry exhibit no identifiable trend or relationship to each other. Many TOC values for all wells are below the $1 \mathrm{mg} / \mathrm{L}$ detection limit, and values above the detection limit typically range between 2 and $3 \mathrm{mg} / \mathrm{L}$. Such a range is within what is expected for uncontaminated groundwaters (Hem 1986; Drever 1988). It is also typical of the range noted for uncontaminated groundwaters that occur elsewhere on the Oak Ridge Reservation (HSW 1991 a-e). Variations in TOC for a well are not correlated with variations in the summed chlorinated and aromatic VOC values in that well. In all case variations in summed chlorinated and aromatic VOC values are small, with the values at or near $0 \mu \mathrm{g} / \mathrm{L}$.

Based on the factors discussed above, the statistically significant changes in TOC noted for wells at the Kerr Hollow Quarry are interpreted to be false positives, and to not represent a release of contaminants into groundwater from the site.

\section{Evaluation of TOX Data}

Total organic halide measurements were obtained from groundwater monitoring wells at Kerr Hollow Quarry (Fig. 12 ) beginning in CY 1986. The mean, median, and variance of TOX measurements are illustrated in Fig. 18. The data indicate that (1) much of the data for all wells is less than the $10 \mu \mathrm{g} / \mathrm{L}$ detection limit, and because of this, the median values for all wells are at the detection limit, (2) three of the seven wells at the site have extreme outliers that range up to $1900 \mu \mathrm{g} / \mathrm{L}$, (3) two of the downgradient wells (GW-142 and GW-144) have extreme outliers that range up to $\sim 400 \mu \mathrm{g} / \mathrm{L}$, and (4) one of the downgradient wells (GW-231) has extreme outliers that range to $-1900 \mu \mathrm{g} / \mathrm{L}$.

Trend plots for TOX measurements from the upgradient and downgradient wells at Kerr Hollow Quarry are illustrated in Figs. 19a through 22a. For comparison, trend plots of summed chlorinated VOCs are presented in Figs. 19b through 22b. Data for TOX and summed chlorinated VOC measurements for the upgradient and downgradient monitoring wells is summarized and discussed in the following sections.

GW-145. The majority of TOX values for upgradient well GW-159 were less than 25 $\mu \mathrm{g} / \mathrm{L}$ (Fig. 19a). Samples obtained during CY $1986 \mathrm{range}$ from $\sim 25 \mu \mathrm{g} / \mathrm{L}$ to $\sim 220 \mu \mathrm{g} / \mathrm{L}$. Subsequent to 1986 TOX values greater than $25 \mu \mathrm{g} / \mathrm{L}$ were not obtained, which suggests that the results for CY 1986 represent spurious analytical data. Summed chlorinated VOCs for well GW-145 during the period 1987 through 1990 are $0 \mu \mathrm{Hg} / \mathrm{L}$ (Fig. 19b). No consistent correlation or temporal pattern in TOX and summed chlorinated VOC values is noted, suggesting that the variability observed likely represents analytical uncertainties. 
GW-142. With one exception, all TOX values for downgradient well GW-142 were less than $50 \mu \mathrm{g} / \mathrm{L}$ (Fig. 20a), and the majority of values are below the detection limit of $10 \mu \mathrm{g} / \mathrm{L}$. Samples obtained during CY 1986 range from the detection limit of 10 $\mu \mathrm{g} / \mathrm{L}$ to $-380 \mu \mathrm{g} / \mathrm{L}$. Subsequent to 1986 no TOX values greater than $\sim 40 \mu \mathrm{g} / \mathrm{L}$ were obtained, which suggests that the results for CY 1986 represent spurious analytical data. Summed chlorinated VOCs ior well GW-142 during the period 1987 through 1990 range between 0 and $1 \mu \mathrm{g} / \mathrm{L}$ (Fig. 20b). No consistent correlation or temporal pattern in TOX and summed chlorinated VOC values is noted, suggesting that the variability observed likely represents analytical uncertainties.

GW-144. With three exceptions, ail TOX values for downgradient well GW-144 were less than $-20 \mu \mathrm{g} / \mathrm{L}$ (Fig. 21a). Samples obtained during CY 1986 range from the detection limit of $10 \mu \mathrm{g} / \mathrm{L}$ to $\sim 80 \mu \mathrm{g} / \mathrm{L}$. Subsequent to 1986 only one TOX value exceeded $\sim 20 \mu \mathrm{g} / \mathrm{L}$, suggesting that the results for CY 1986 represent spurious analytical data. Summed chlorinated VOCs for well GW-144 during the period 1987 through 1990 range from 0 to $-3 \mu \mathrm{g} / \mathrm{L}$ (Fig. 21b), with only two results being greater than $0 \mu \mathrm{g} / \mathrm{L}$. No consistent correlation or temporal pattern in TOX and summed chlorinated VOC values is noted, suggesting that the variability observed likely represents analytical uncertainties.

GW-231. With one exception, all TOX values for downgradient well GW-231 were less than $-50 \mu \mathrm{g} / \mathrm{L}$ (Fig. 22a), and the majority of values are below the detection limit of $10 \mu \mathrm{g} / \mathrm{L}$. Samples obtained during CY 1986 range from the detection limit of 10 $\mu \mathrm{g} / \mathrm{L}$ to $-1750 \mu \mathrm{g} / \mathrm{L}$. Subsequent to 1986 no IOX values greater than $50 \mu \mathrm{g} / \mathrm{L}$ were obtained, which suggests that the results for CY 1986 represent spurious analytical data. During the period 1989 through 1990, TOX values were less than $-20 \mu \mathrm{g} / \mathrm{L}$. Summed chlorinated VOCs for well GW-231 during the period 1987 through 1990 range from 0 to $1 \mu \mathrm{g} / \mathrm{L}$ (Fig. 22b), with only one value above $0 \mu \mathrm{g} / \mathrm{L}$. No consistent correlation or temporal pattern in TOX and summed chlorinated VOC values is noted, suggesting that the variability observed likely represents analytical uncertainties.

Discussion and Summary. Temporal variations in TOX values for both the upgradient and downgradient wells at the Kerr Hollow Quarry exhibit no identifiable trend or relationship to each other. Many TOX values for all wells are below the $10 \mu \mathrm{g} / \mathrm{L}$ detection limit, and values above the detection limit typically range between 10 and $50 \mu \mathrm{g} / \mathrm{L}$. Variations in TOX for a well are not correlated with variations in the summed chlorinated and aromatic VOC values in that well. In all cases variations in summed chlorinated and aromatic VOC values are small, with the values at or near $0 \mu \mathrm{g} / \mathrm{L}$.

Based on the factors discussed above, the statistically significant changes in TOX noted for wells at the Kerr Hollow Quarry are interpreted to be false positives, and to not represent a release of contaminants into groundwater from the site. 


\section{REFERENCES}

Drever, J. I. 1988. The Geochemistry of Natural Waters (Seconc Edition). Prentice Hall, Englewood Cliffs, New Jersey.

Hem, J. D. 1986. Study and interpretation of the chemical chari zteristics of natural water. U. S. Geological Survey Water Supply Paper 2254.

HSW, Inc. 1991b. Groundwater quality assessment for the Be r Creek hydrologic regime at the Y-12 Plant, 1990: Data interpretation anc proposed program modifications. $Y / S U B / 91-Y P 507 C / 1$ Part 2.

HSW, Inc. 1991c. Groundwater quality assessment for the UF jer East Fork Poplar Creek hydrologic regime at the Y-12 Plant, 1990: Grou .dwater quality data and calculated rate of contaminant migration. Y/SUB/91. YP507C/2 Part 1.

HSW, Inc. 1991d. Groundwater quality assessment for the U. per East Fork Poplar Creek hydrologic regime at the $Y-12$ Plant, 1990: $D z$ :a interpretation and proposed program modifications. Y/SUB/91-YP507C/2 : art 2.

HSW, Inc. 1991e. Groundwater quality assessment for the Ridge Security Pits Hazardous Waste Disposal Unit at the Y-12 Plant, 1990. :/SUB/91-YP507C/3. 
ORNL-DWO OIM-1060S

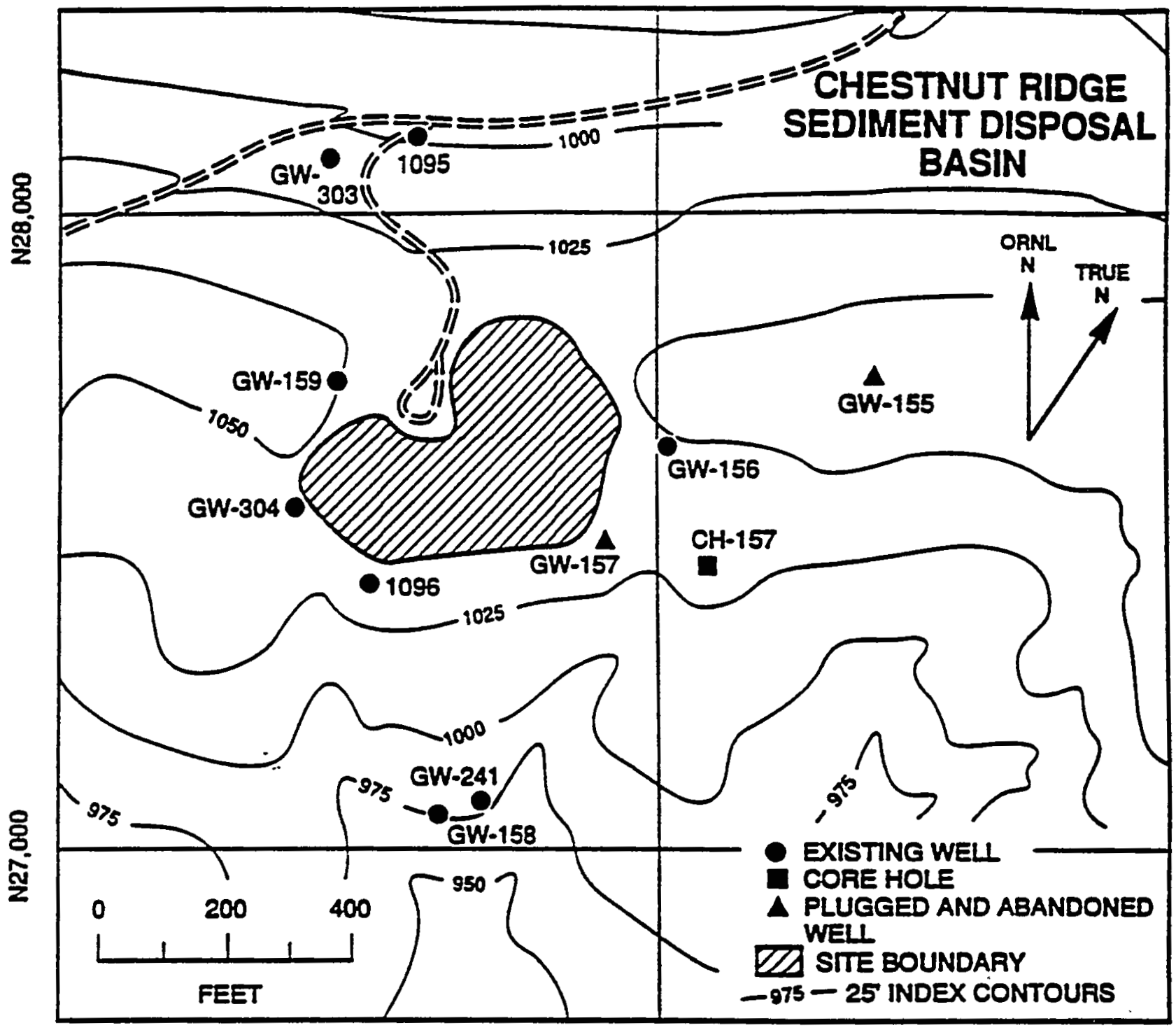

$E 64,000$

Figure 1. Location map for the Chestnut Ridge Sediment Disposal Basin. Upgradient monitoring well is GW-159. Downgradient monitoring wells are GW-155, GW-156, and GW-157. 


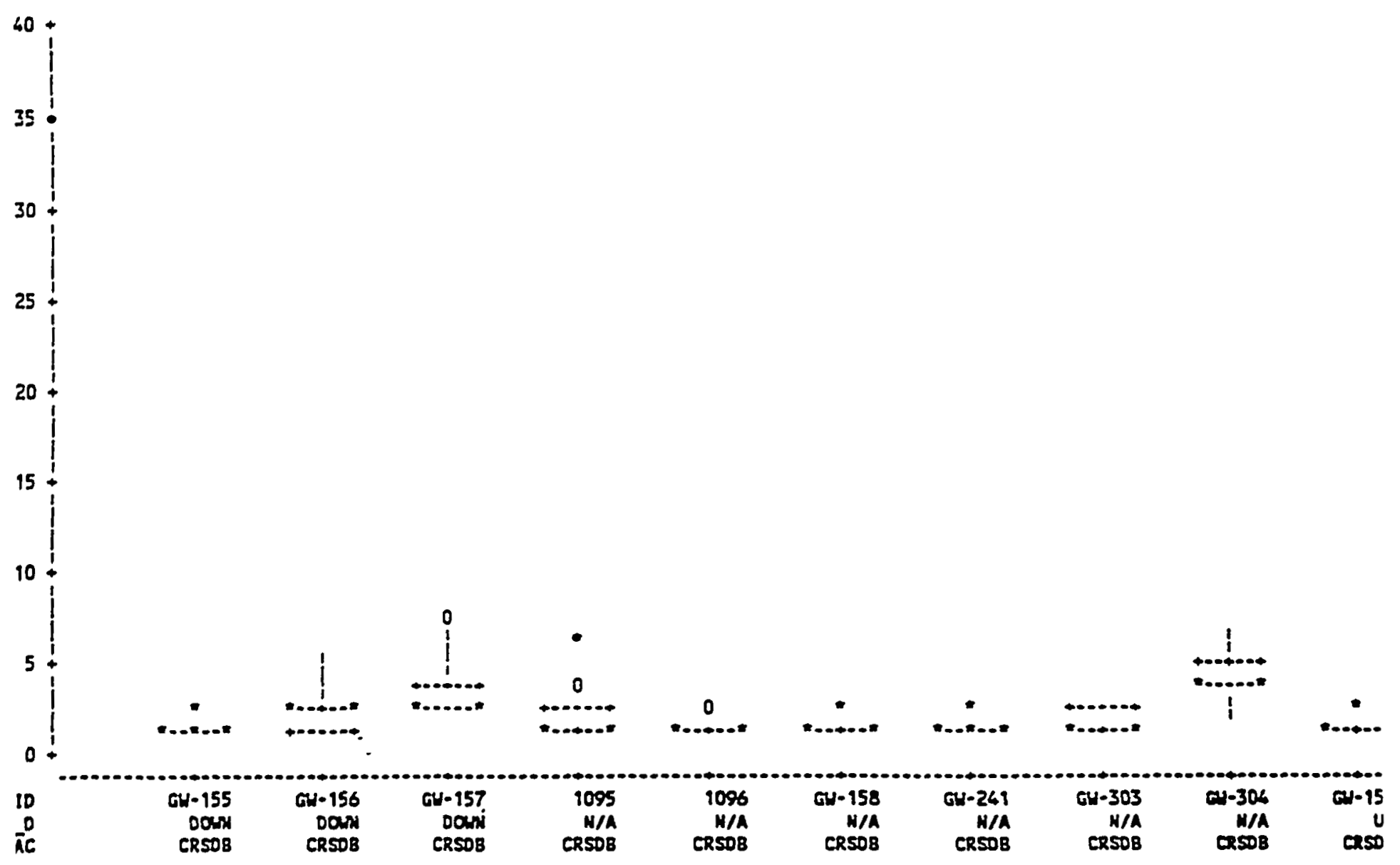

Figure 2. Statistical summary of TOC data for groundwater monitoring wells at the Chestnut Ridge Sediment Disposal Basin. Box outlines represent the 25th percentile (bottom of box), median (middle line terminated with asterisks), and 75th percentile (top of box) of the data set for a individual well. Mean is indicated by central plus sign ( $t$ ) within the box. Central lines or tails extending above and below the box indicate the range of outliers within 1.5 interquartile ranges below the 25th percentile and above the 75th percentile. Outliers beyond this range are illustrated by zero $(0)$ if they are within 3 interquartile ranges. More extreme outliers are illustrated by an asterisk $(*)$. Because of scale, some boxes appear incomplete. 


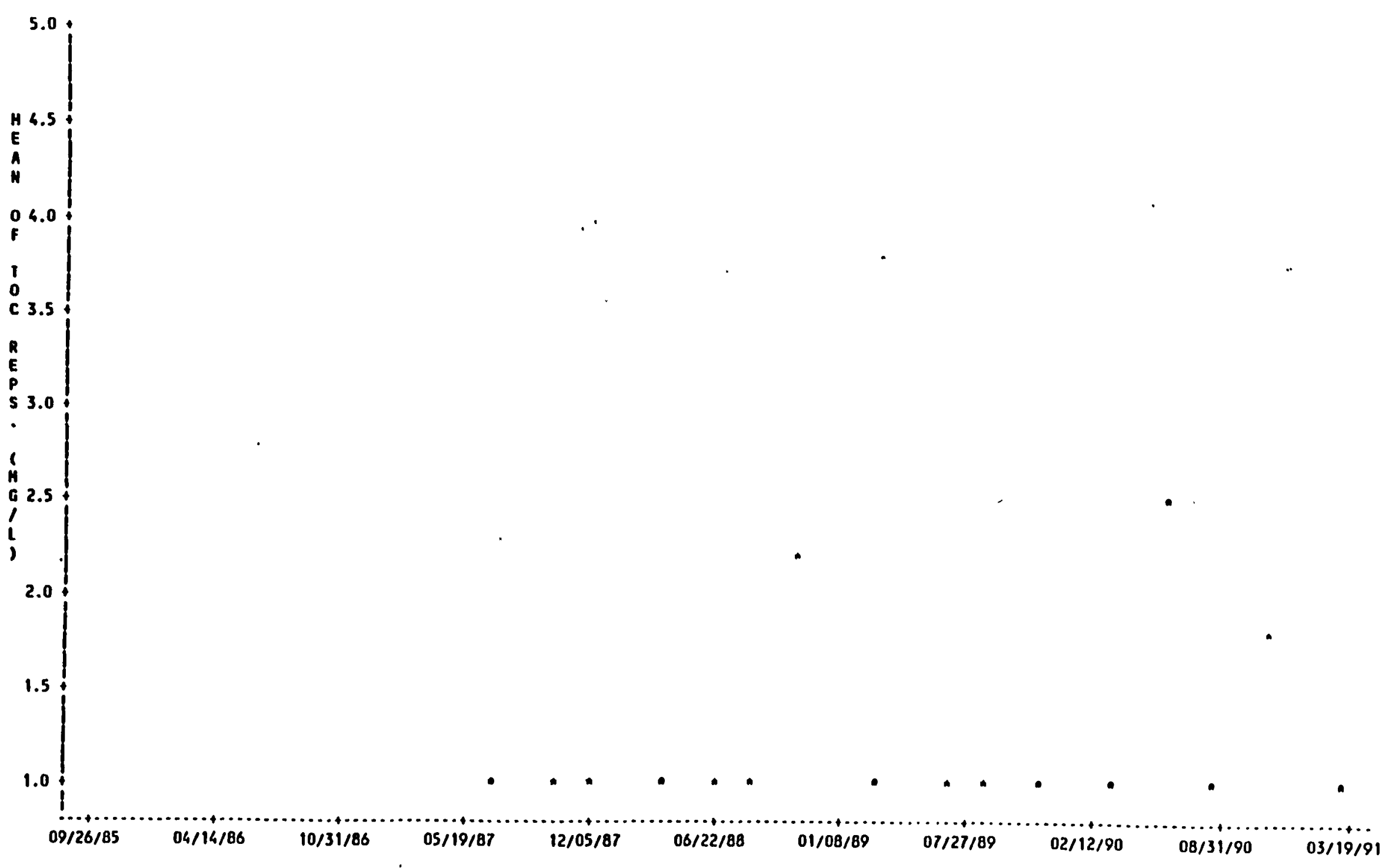

Figure 3a. Plot of TOC values for upgradient well GW-159 at the Chestnut Ridge Sediment Disposal Basin. Each data point is the mean of four measurements. 


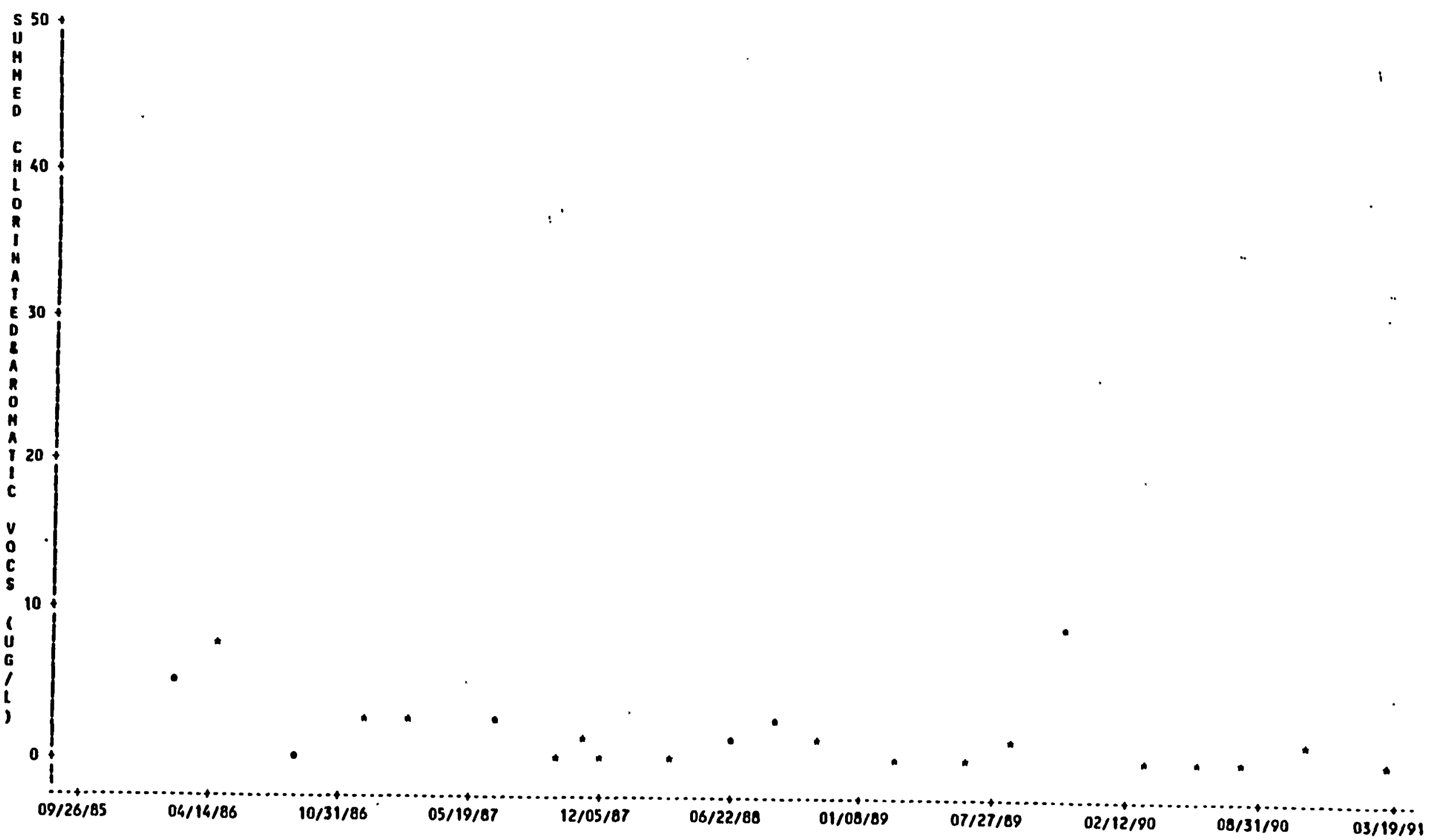
Figure 3b. Plot of summed chlorinated and aromatic VOCs for upgradient well GW-159 at the Chestnut Ridge
Sediment Disposal Basin. Each data point is the mean of four measurements. 


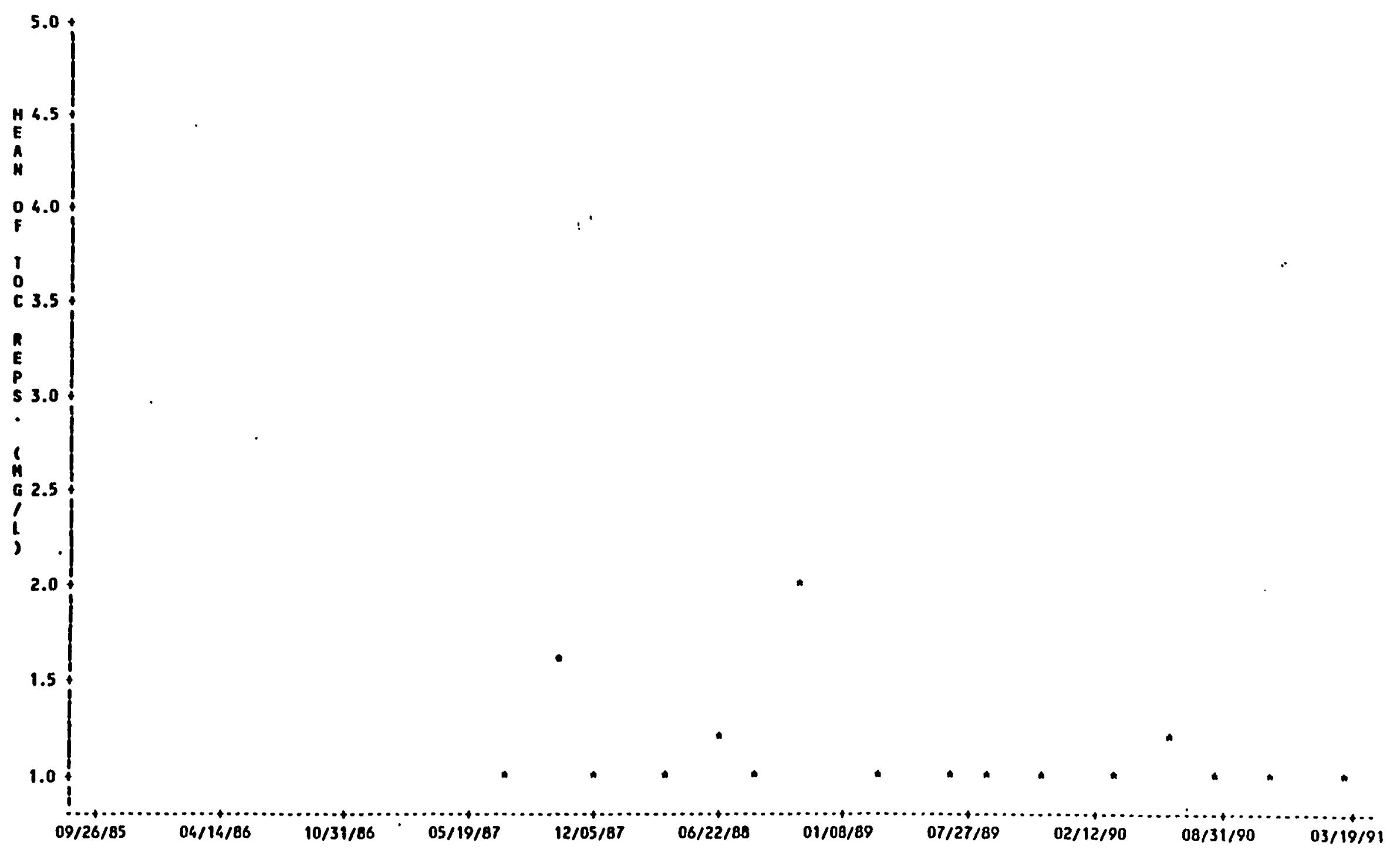

Figure 4a. Plot of TOC values for downgradient well GW-155 at the Chestnut Ridge Sediment Disposal Basin. Each data point is the mean of four measurements. 


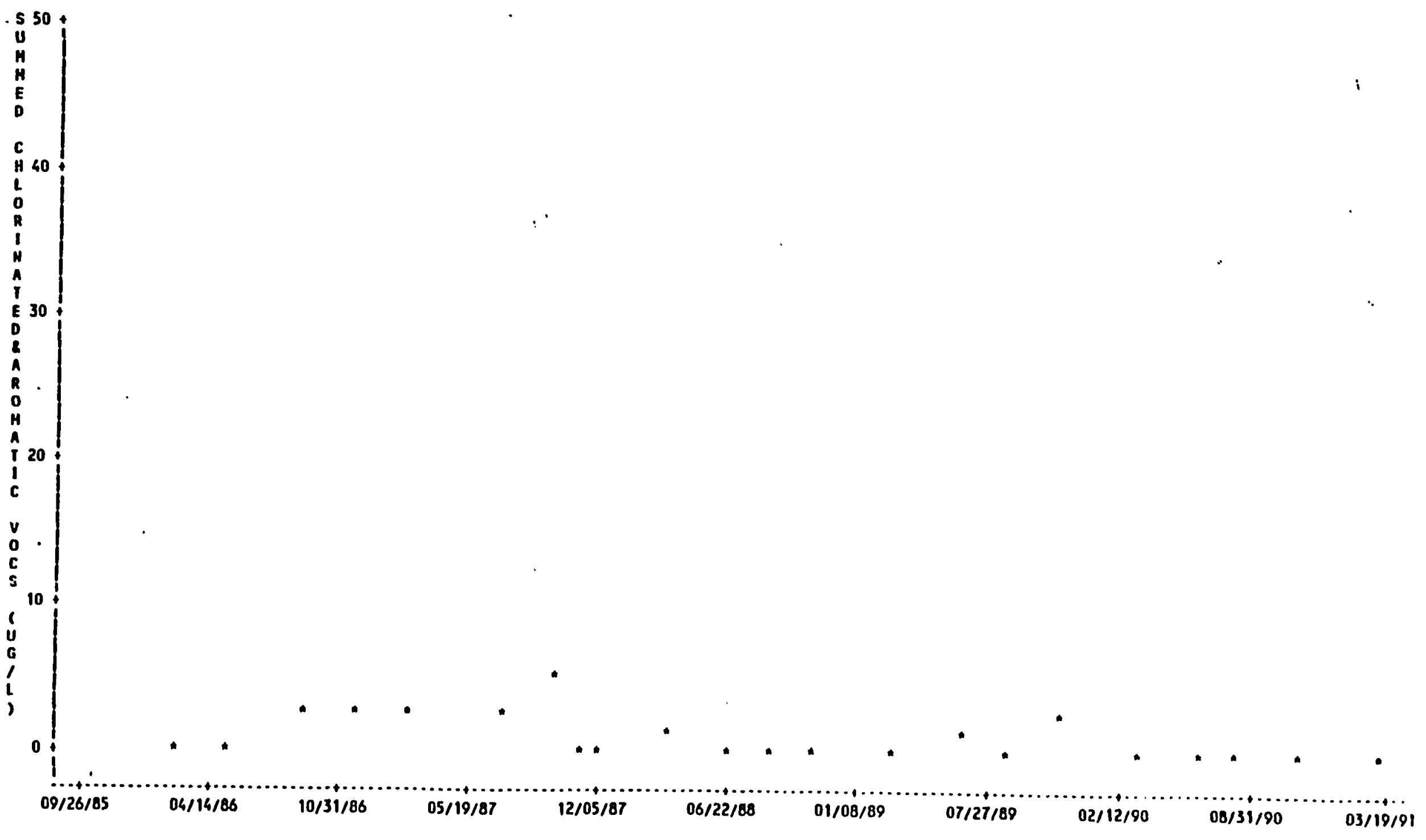

Figure 4b. Plot of summed chlorinated and aromatic VOCs for downgradient well GW-155 at the Chestnut Ridge Sediment Disposal Basin. Each data point is the mean of four measurements. 


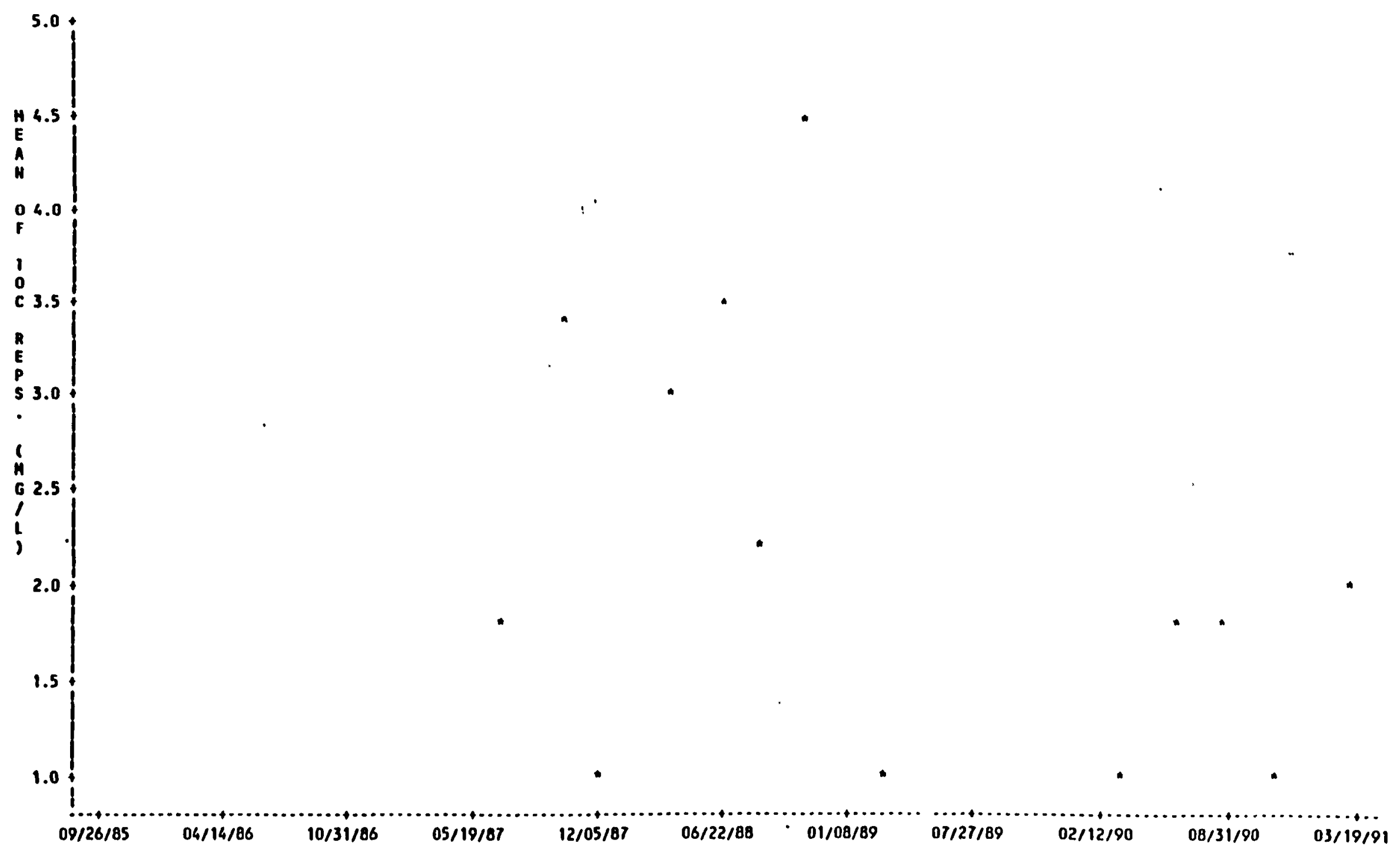

Figure 5a. Plot of TOC values for downgradient well GW-156 at the Chestnut Ridge Sediment Dispus..: Basin. Each data point is the mean of four measurements. 


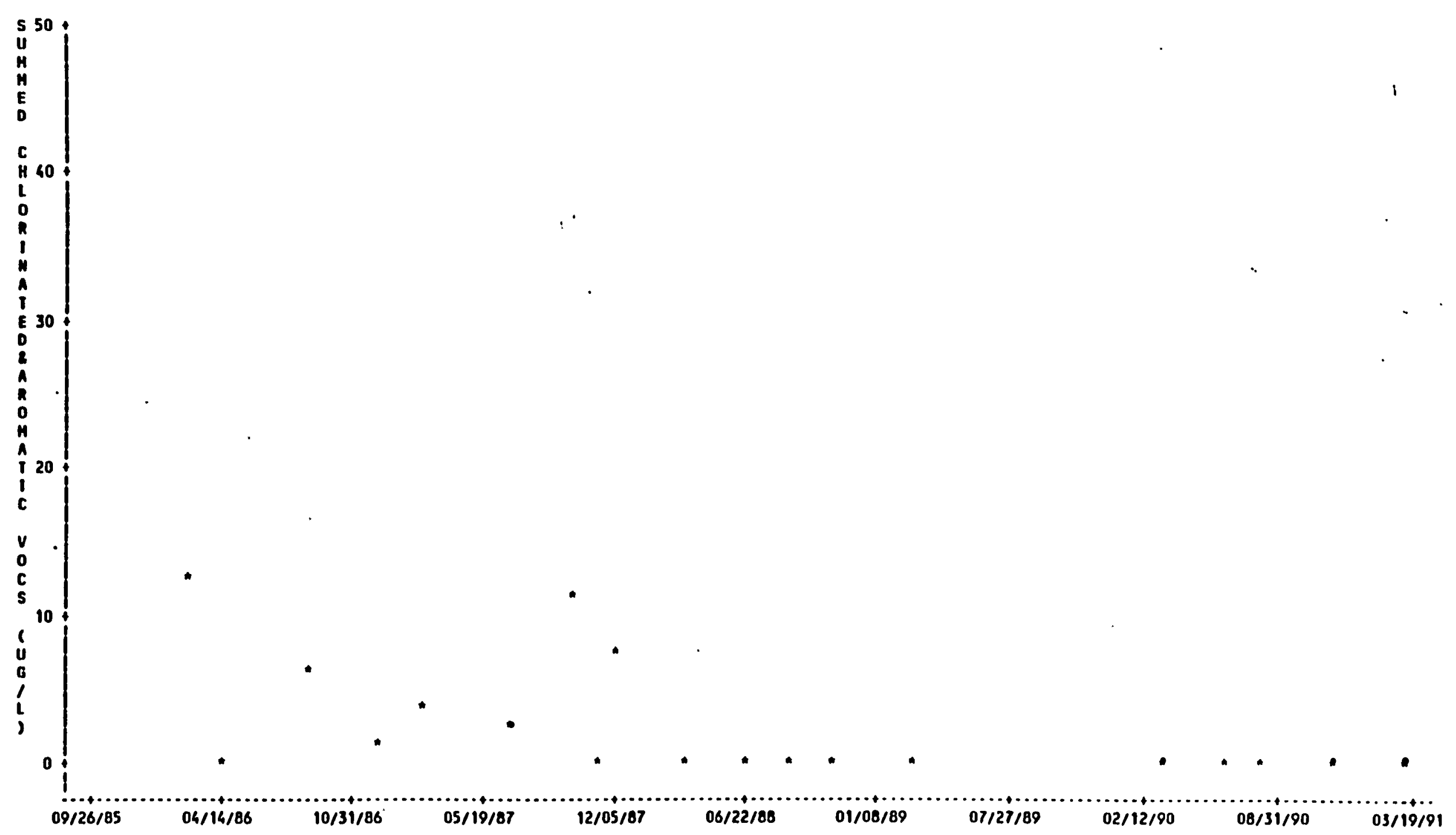

Figure 5b. Plot of summed chlorinated and aromatic VOCs for downgradient well GW-156 at the Chestnut Ridge Sediment Disposal Basin. Each data point is the mean of four measurements. 


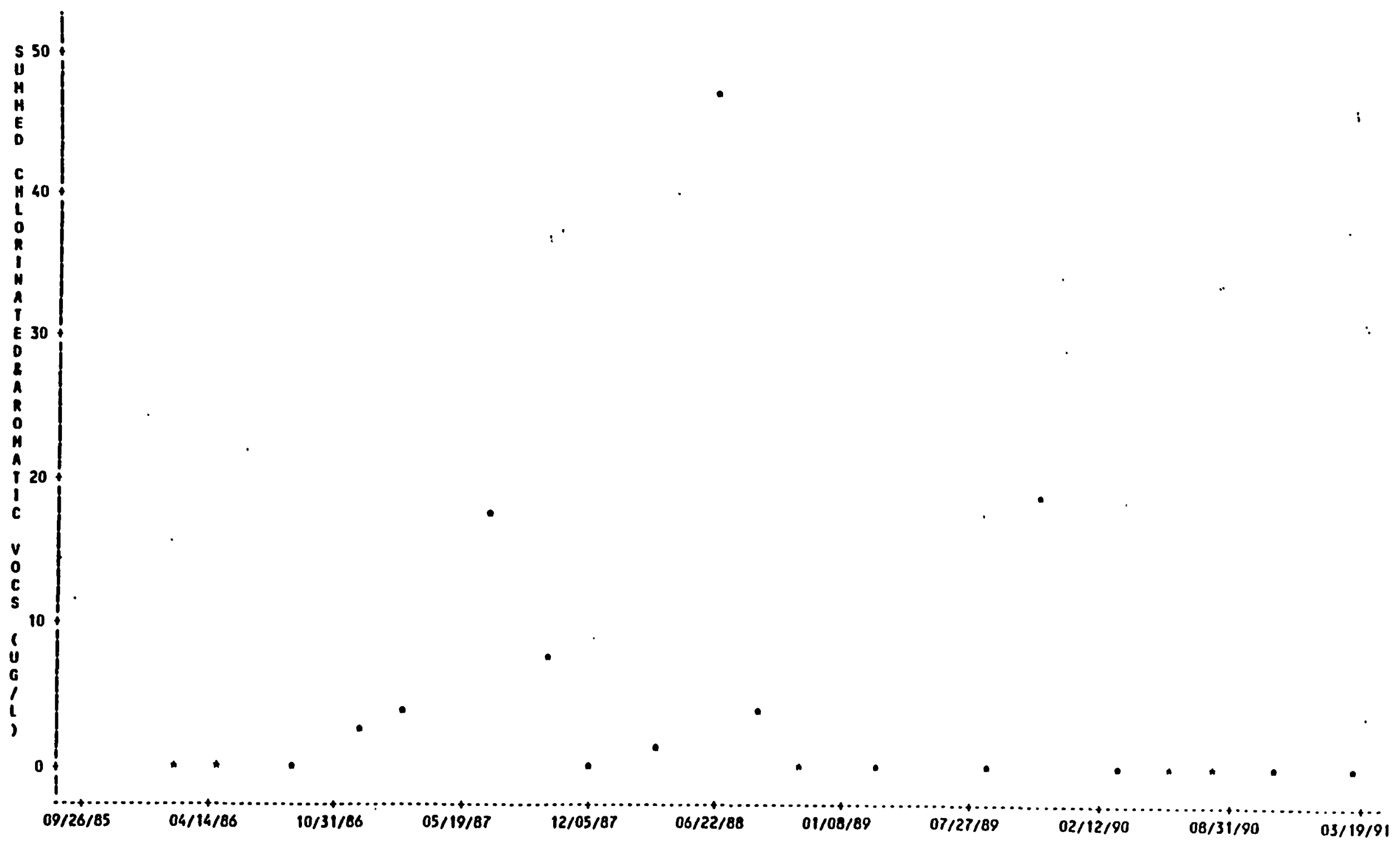

Figure 6b. Plot of summed chlorinated and aromatic VOCs for downgradient well GW-157 at the Chestnut Ridge Sediment Disposal Basin. Each data point is the mean of four measurements. 

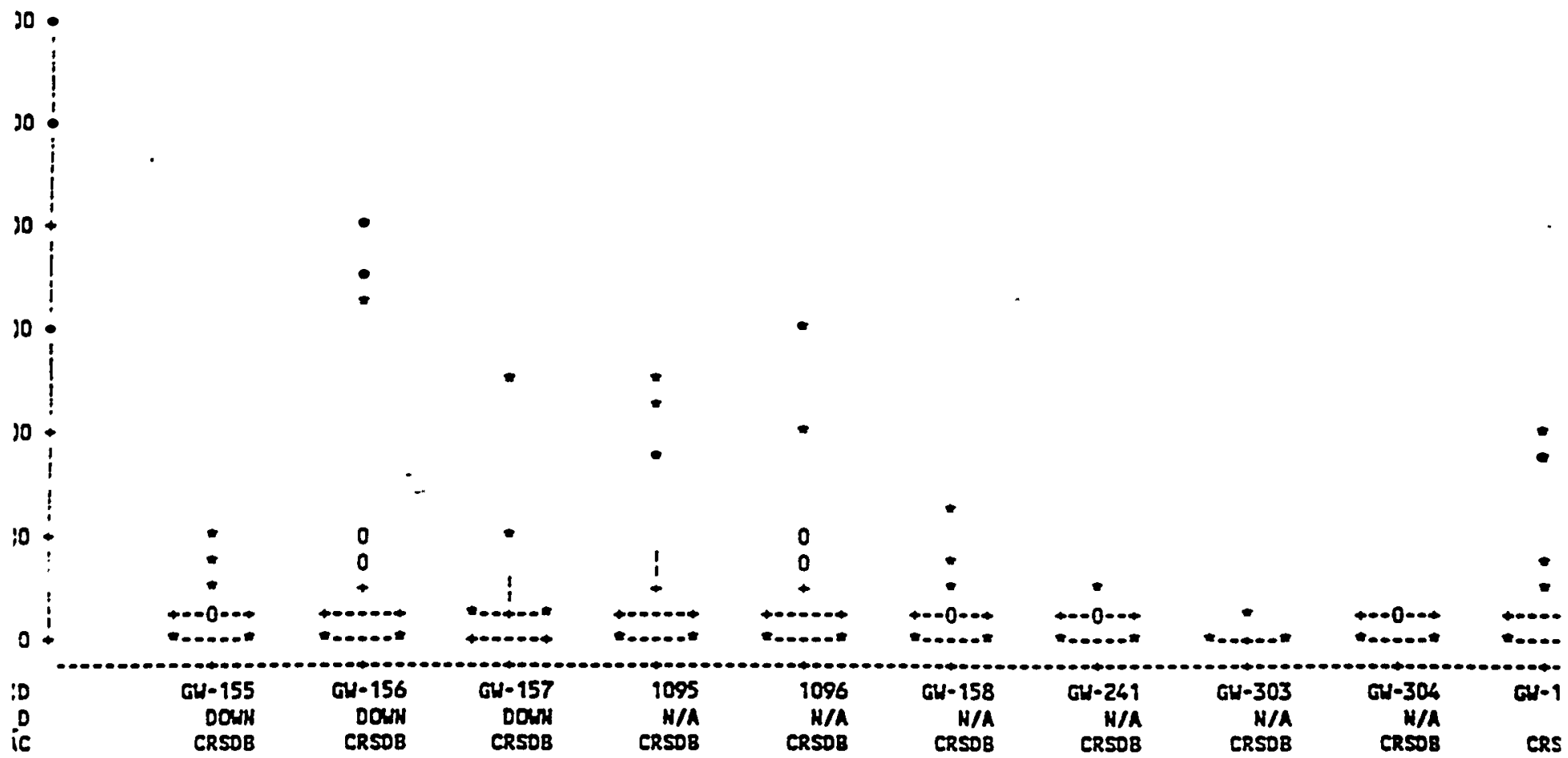

Figure 7. Statistical summary of TOX data for groundwater monitoring wells at the Chestnut Ridge Sediment Disposal Basin. Box outlines represent the 25th percentile (bottom of box), median (middle line terminated with asterisks), and 75th percentile (top of box) of the data set for a individual well. Mean is indicated by central plus sign (+) within the box. Central lines or tails extending above and below the box indicate the range of outliers within 1.5 interquartile ranges below the 25th percentile and above the 75th percentile. Outliers beyond this range are illustrated by zero $(0)$ if they are within 3 interquartile ranges. More extreme outliers are illustrated by an asterisk (*). Because of scale, some boxes appear incomplete. 


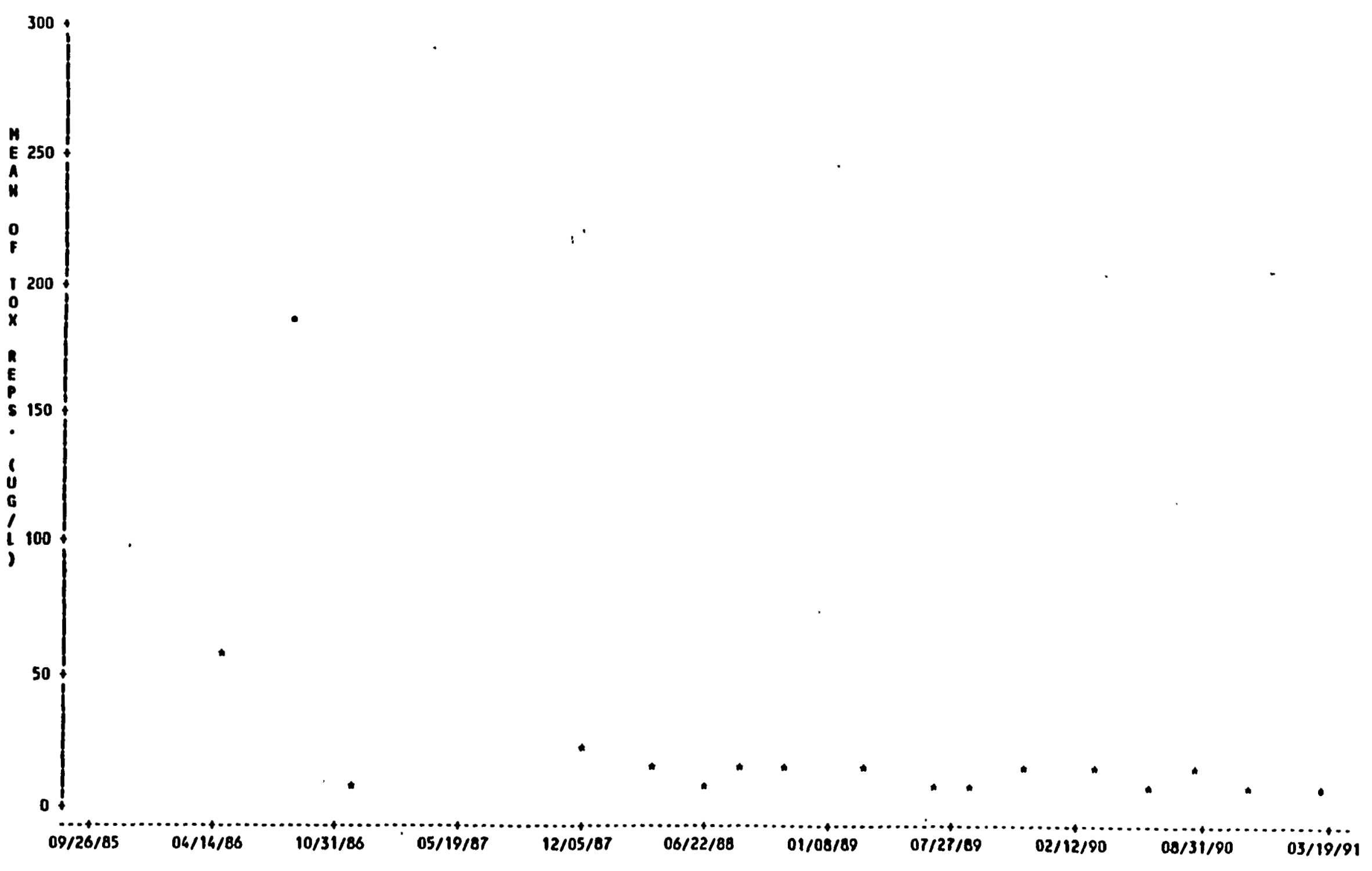

Figure 8a. Plot of TOX values for upgradient well GW-159 at the Chestnut Ridge Sediment Disposal Basin. Each data point is the mean of four measurements. 


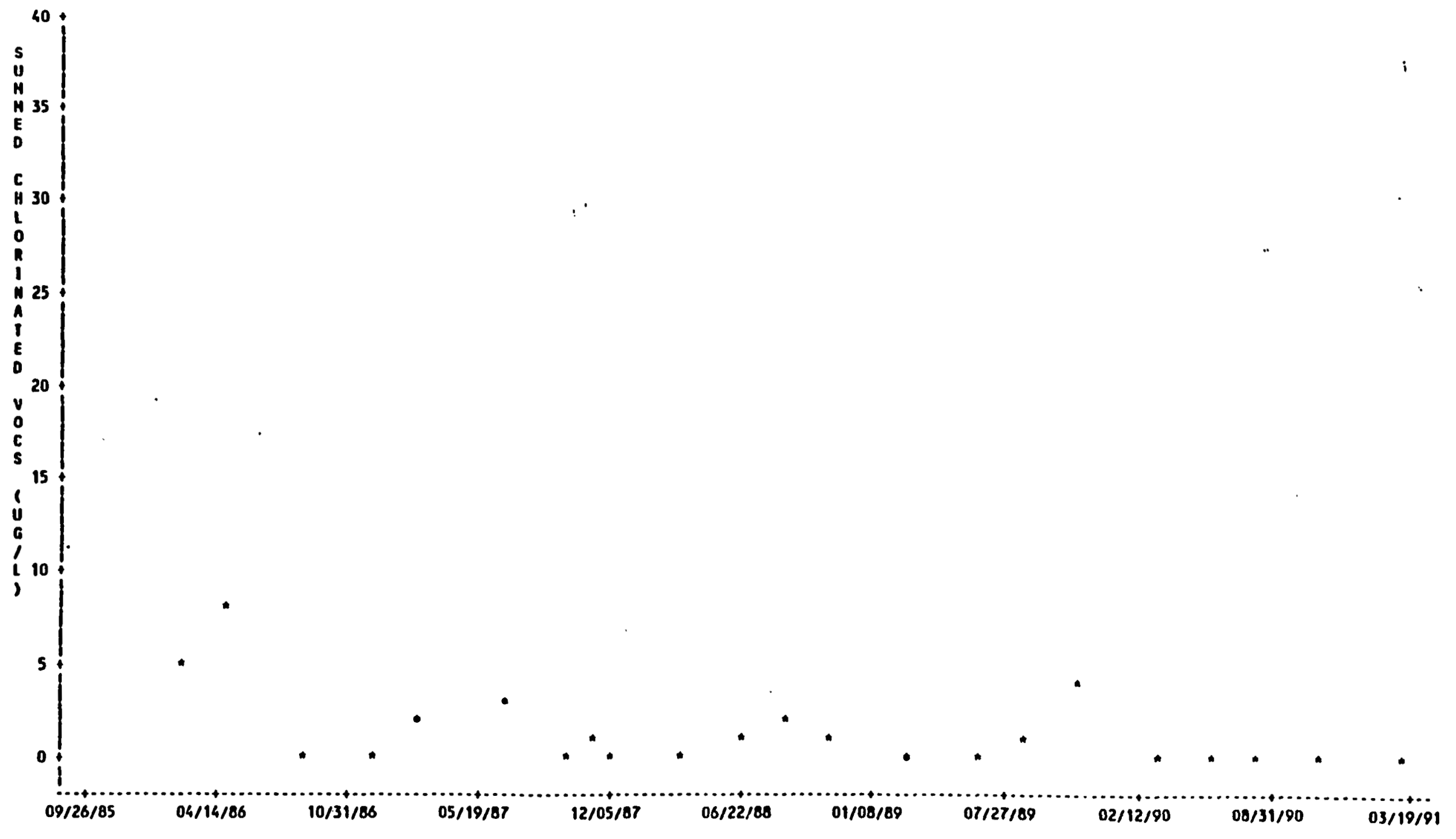

Figure Bb. Plot of summed chlorinated VOCs for upgradient well GW-159 at the Chestnut Ridge Sediment Disposal Basin. Each data point is the mean of four measurements.

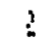




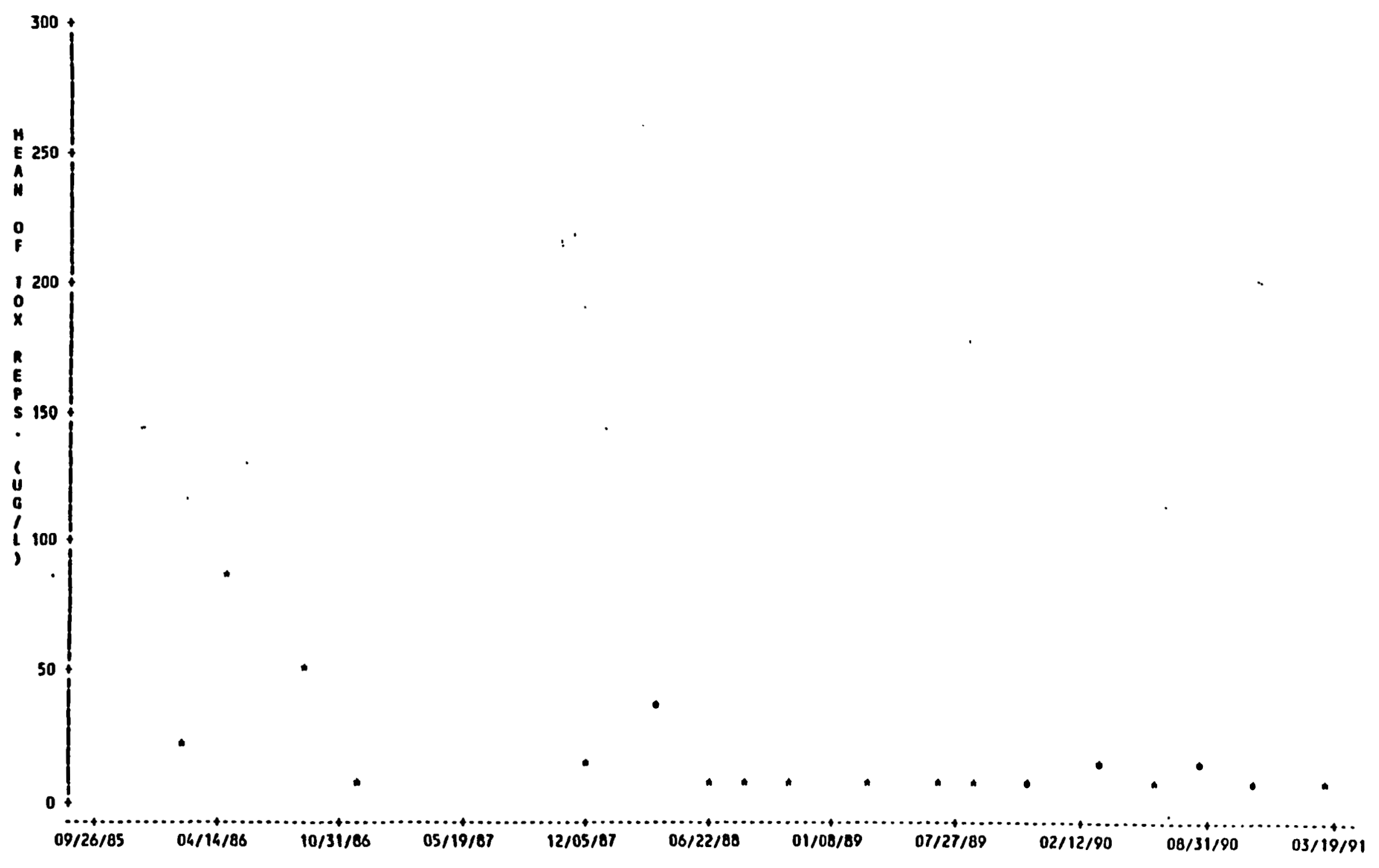

Figure 9a. Plot of TOX values for downgradient well GW-155 at the Chestnut Ridge Sediment Disposal Basin. Each data point is the mean of four measurements. 


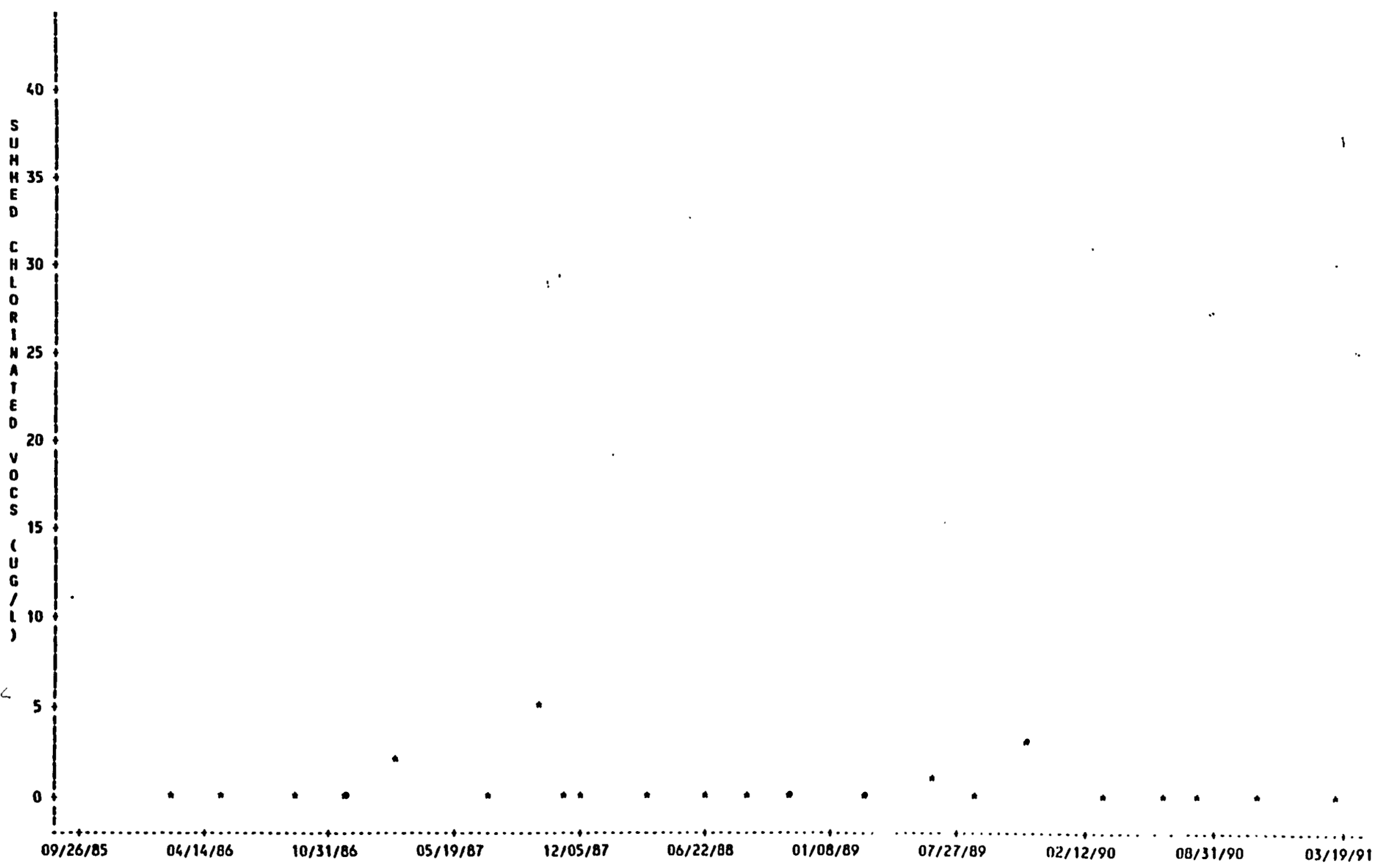

Figure 9b. Plot of summed chlorinated VOCs for downgradient well GW-155 at the Chestnut Ridge Sediment Disposal Basin. Each data point is the mean of four measurements. 


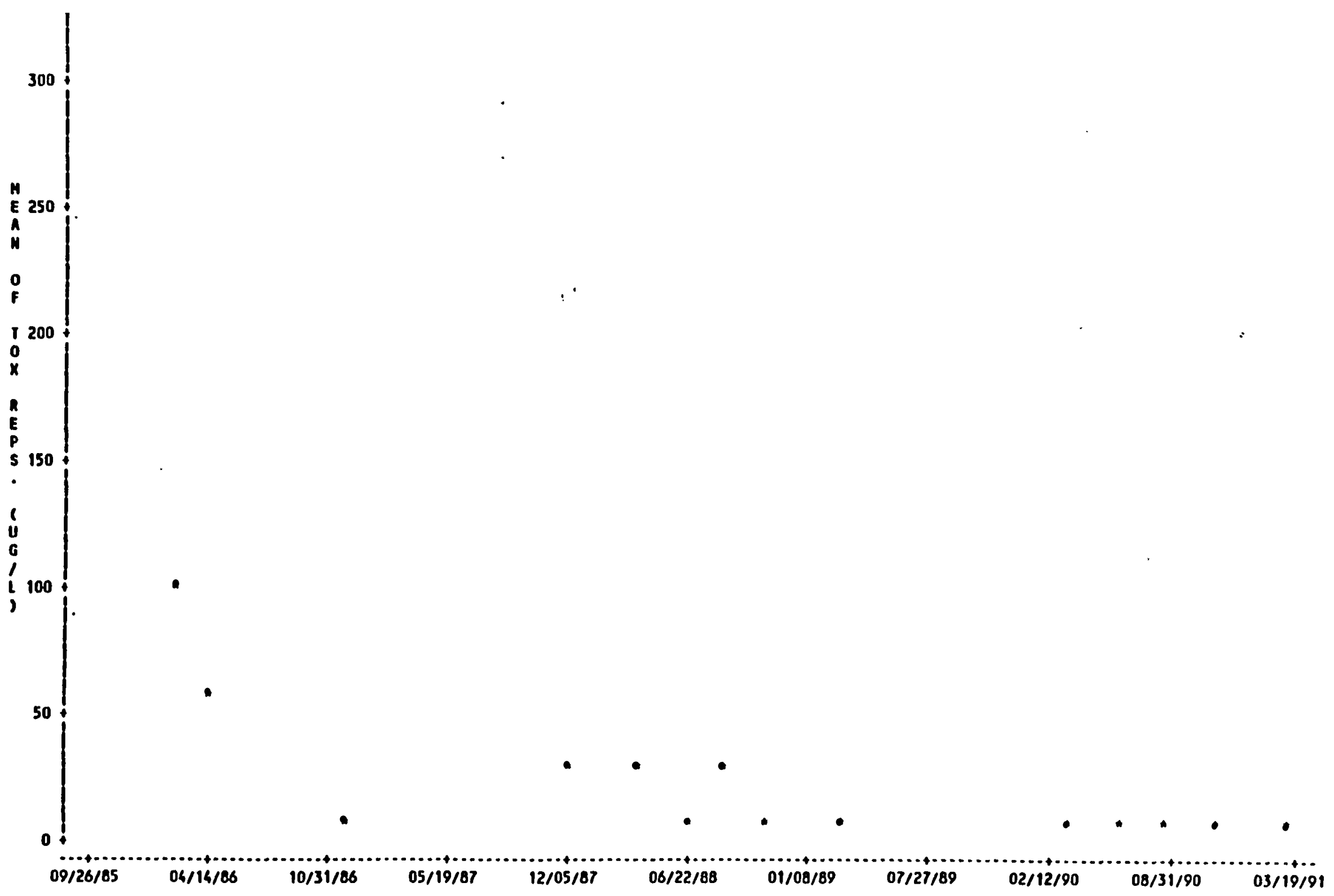

Figure 10a. Plot of TOX values for downgradient well GW-156 at the Chestnut Ridge Sediment Disposal Basin. Each data point is the mean of four measurements. 


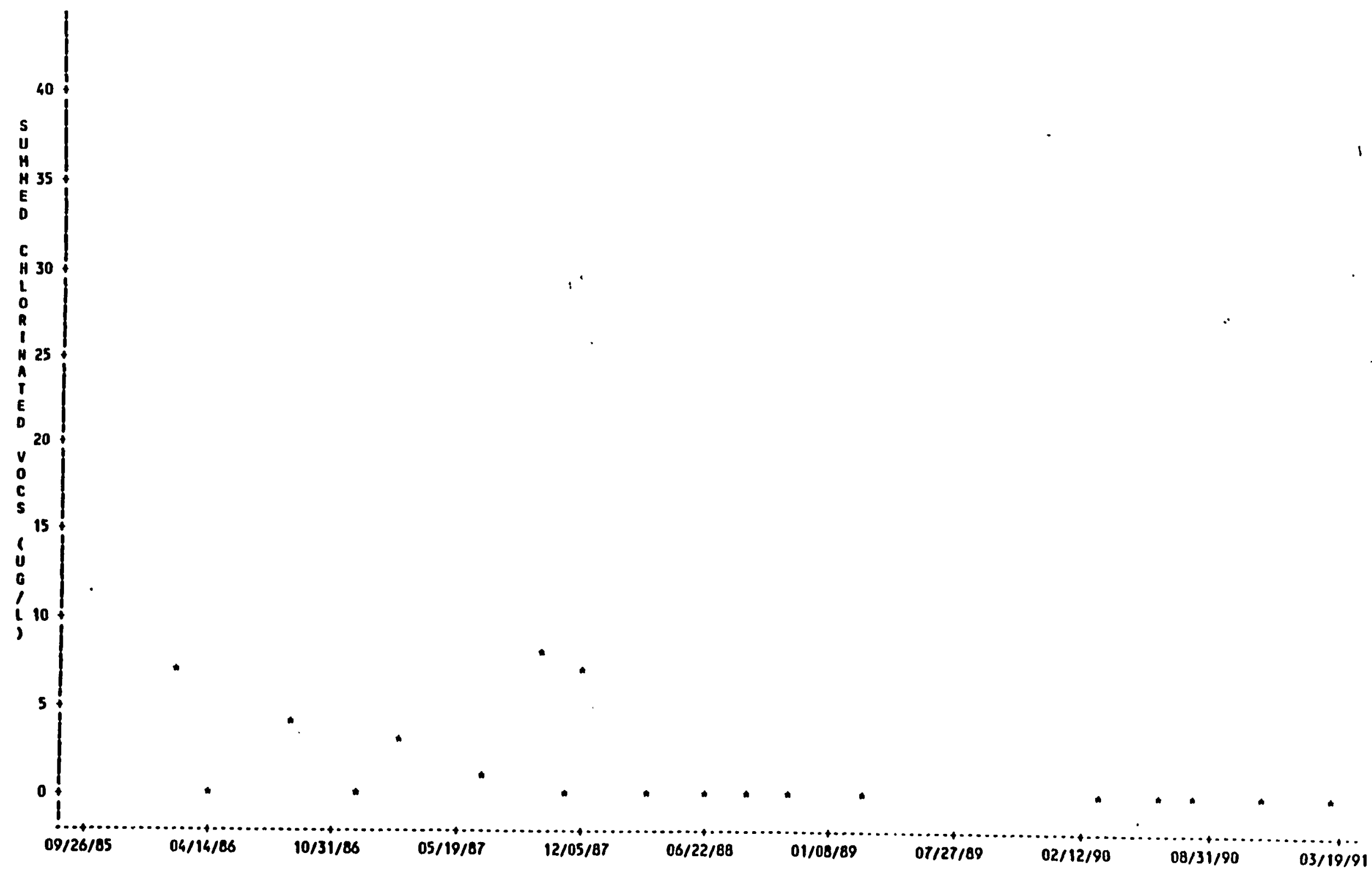

Figure 10b. Plot of summed chlorinated VOCs for downgradient well GW-156 at the Chestnut Ridge Sediment Disposal Basin. Each data point is the mean of four measurements. 


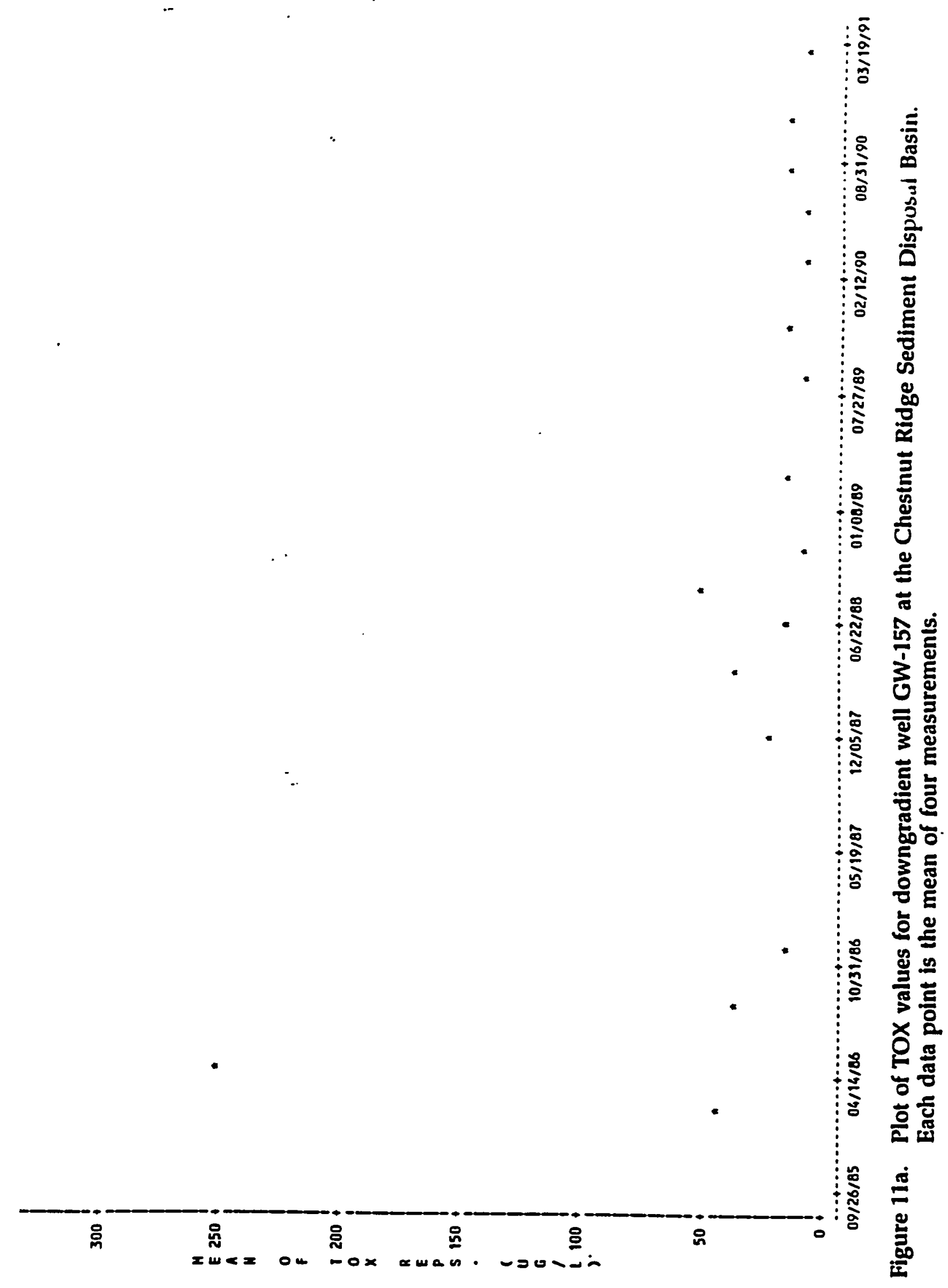




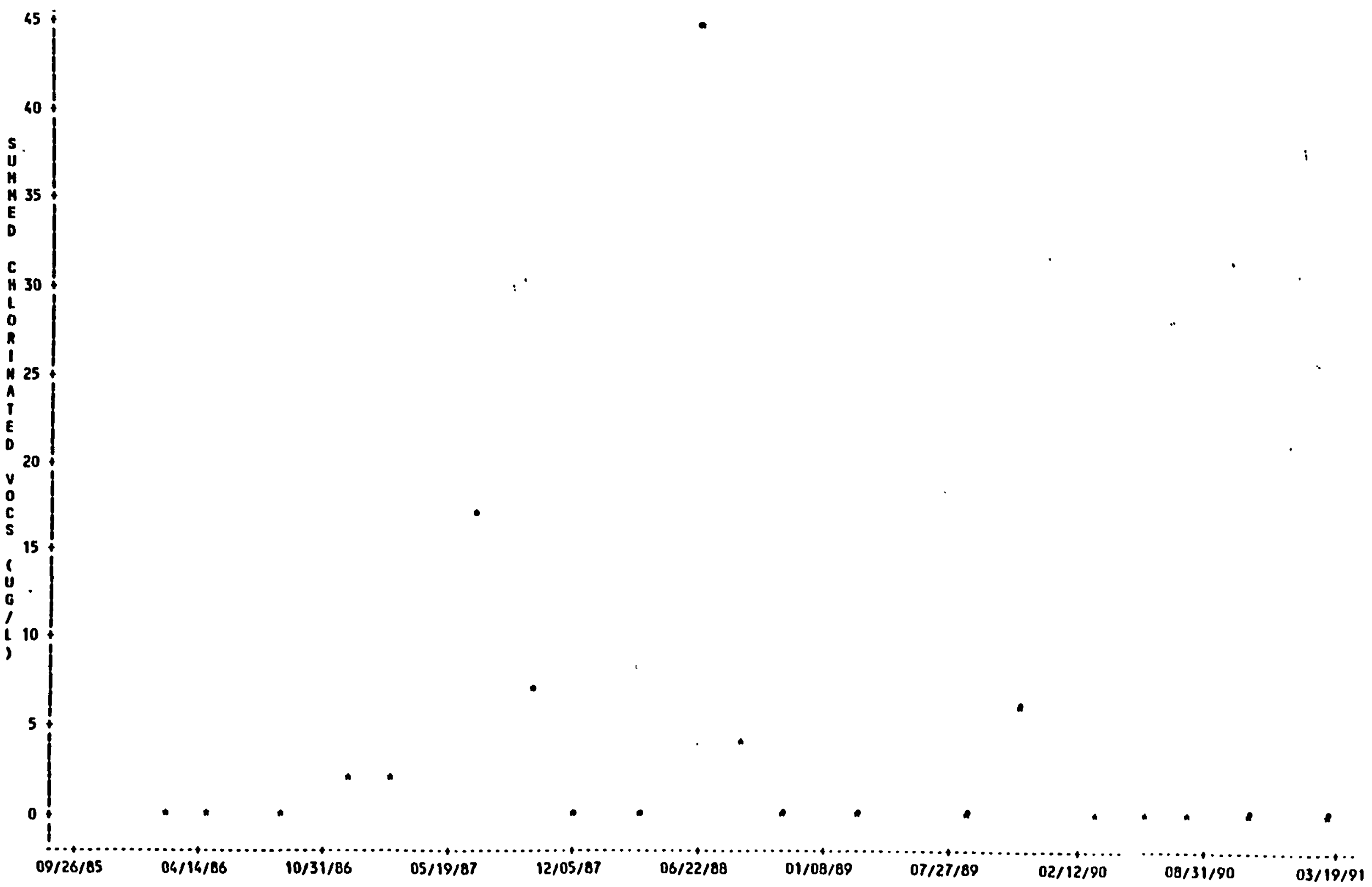

Figure 11b. Plot of summed chlorinated VOCs for downgradient well GW-157 at the Chestnut Ridge Sediment Disposal Basin. Each data point is the mean of four measurements. 
OARLDWO 91M-10500

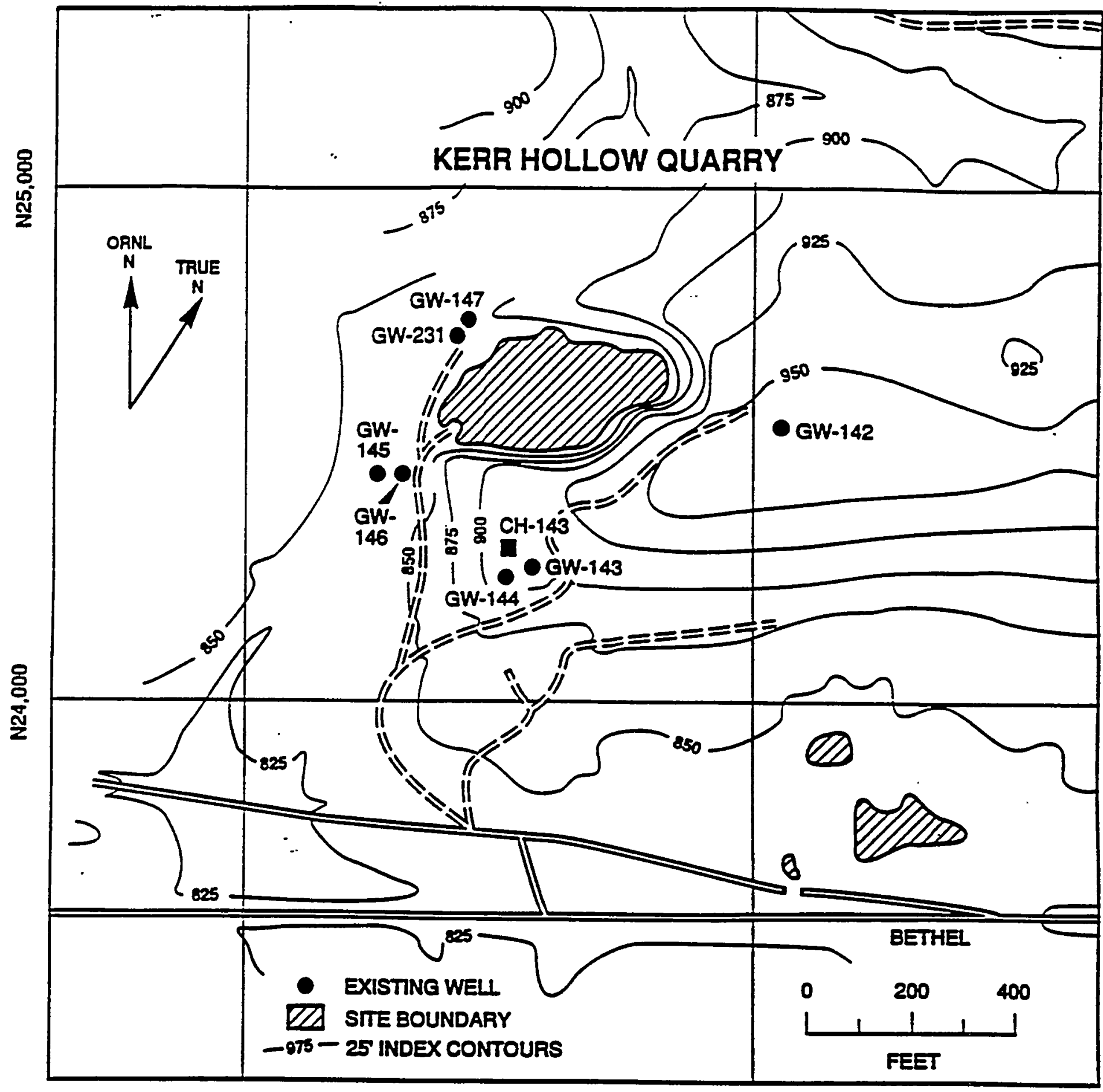

$E 63,000$

$E 64,000$

Figure 12. Location map for the Kerr Hollow Quarry. Upgradient monitoring well is GW-145. Downgradient monitoring wells are GW-142, GW-144, and GW-231. 


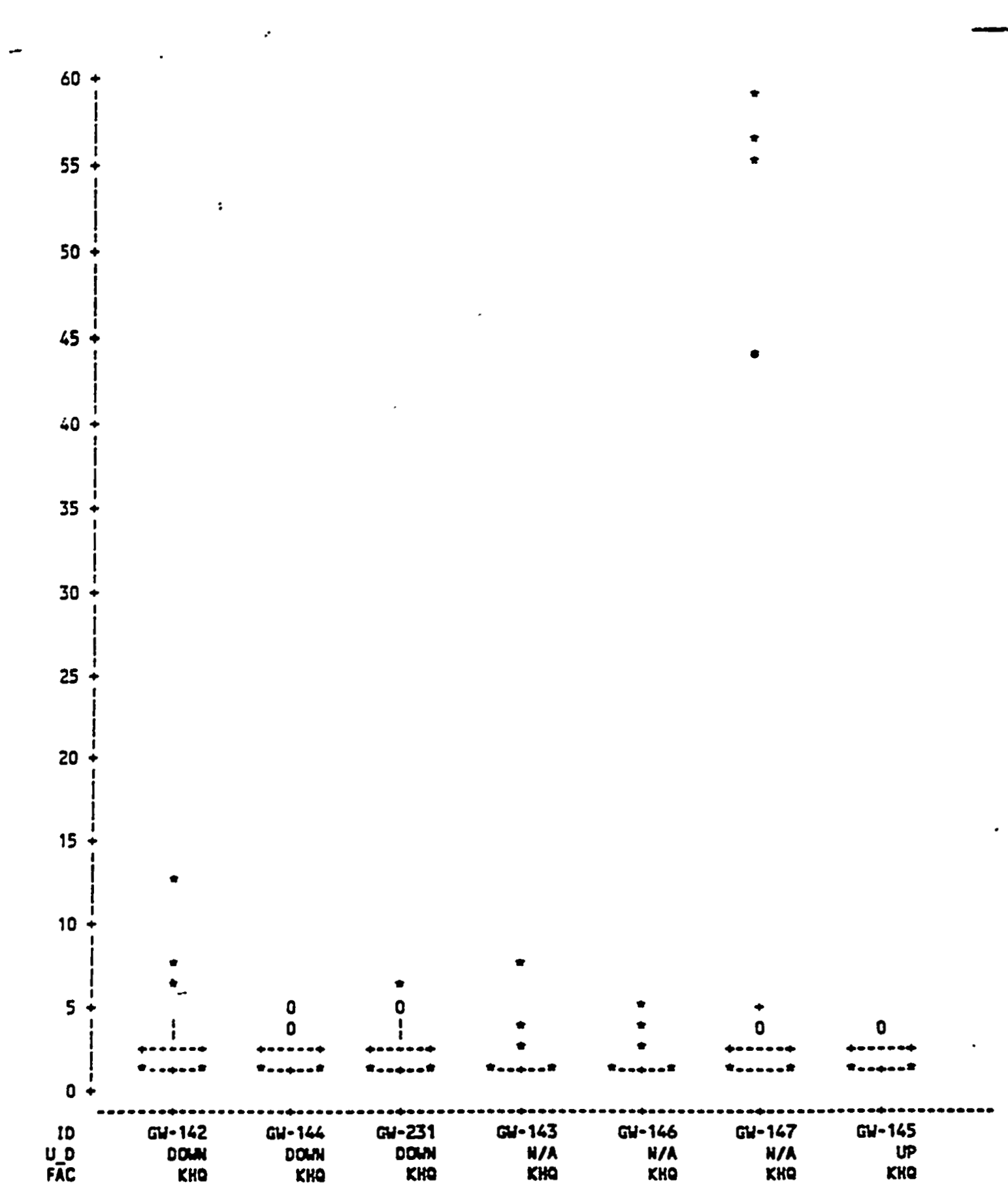

Figure 13. Statistical summary of TOC data for groundwater monitoring wells at the Kerr Hollow Quarry. Box outlines represent the 25th percentile (bottom of box), median (middle line terminated with asterisks), and 75th percentile (top of box) of the data set for a individual well. Mean is indicated by central plus sign ( + ) within the box. Central lines or tails extending above and below the box indicate the range of outliers within 1.5 interquartile ranges below the 25th percentile and above the 75th percentile. Outliers beyond this range are illustrated by zero $(0)$ if they are within 3 interquartile ranges. More extreme outliers are illustrated by an asterisk (*). Because of scale, some boxes appear incomplete. 


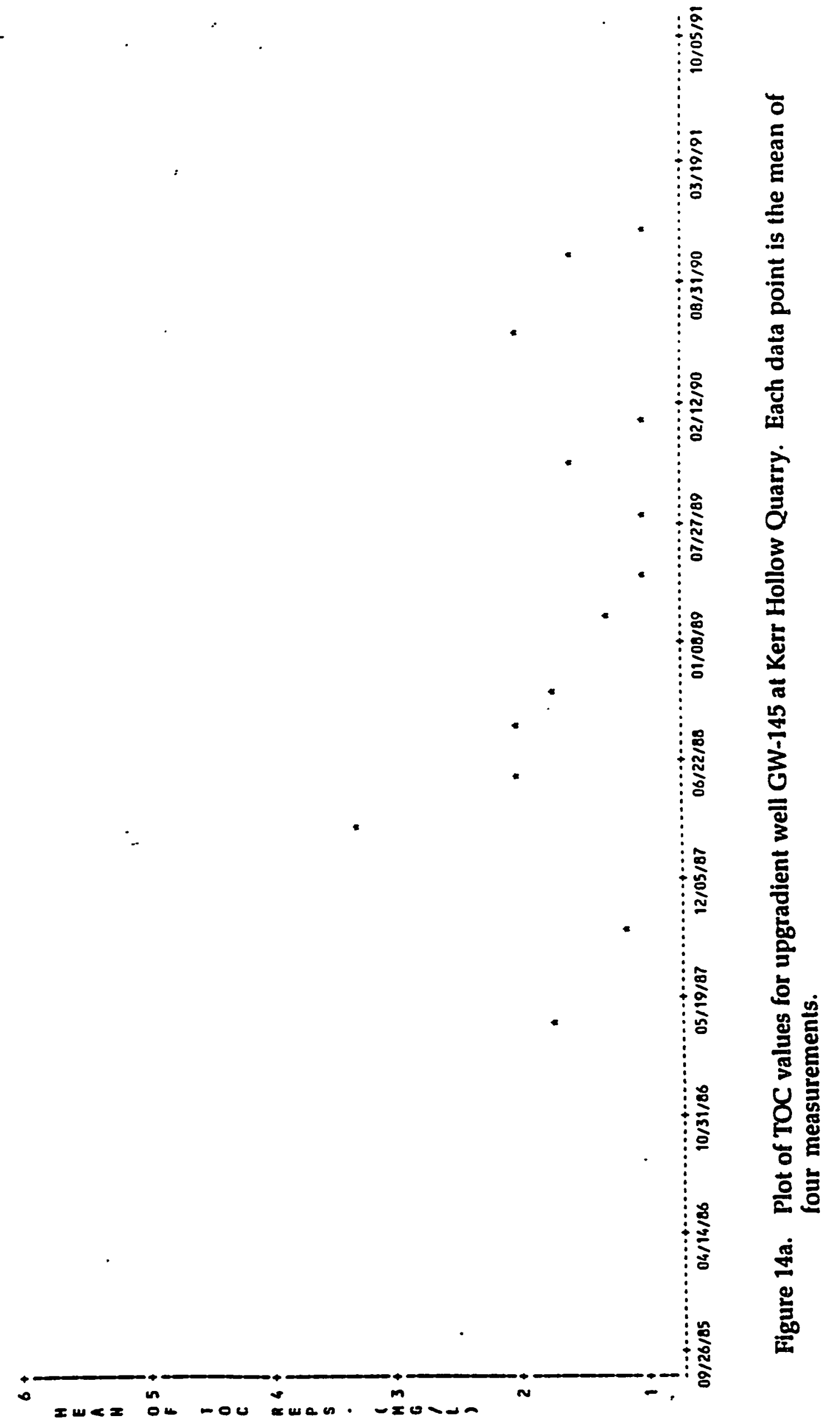




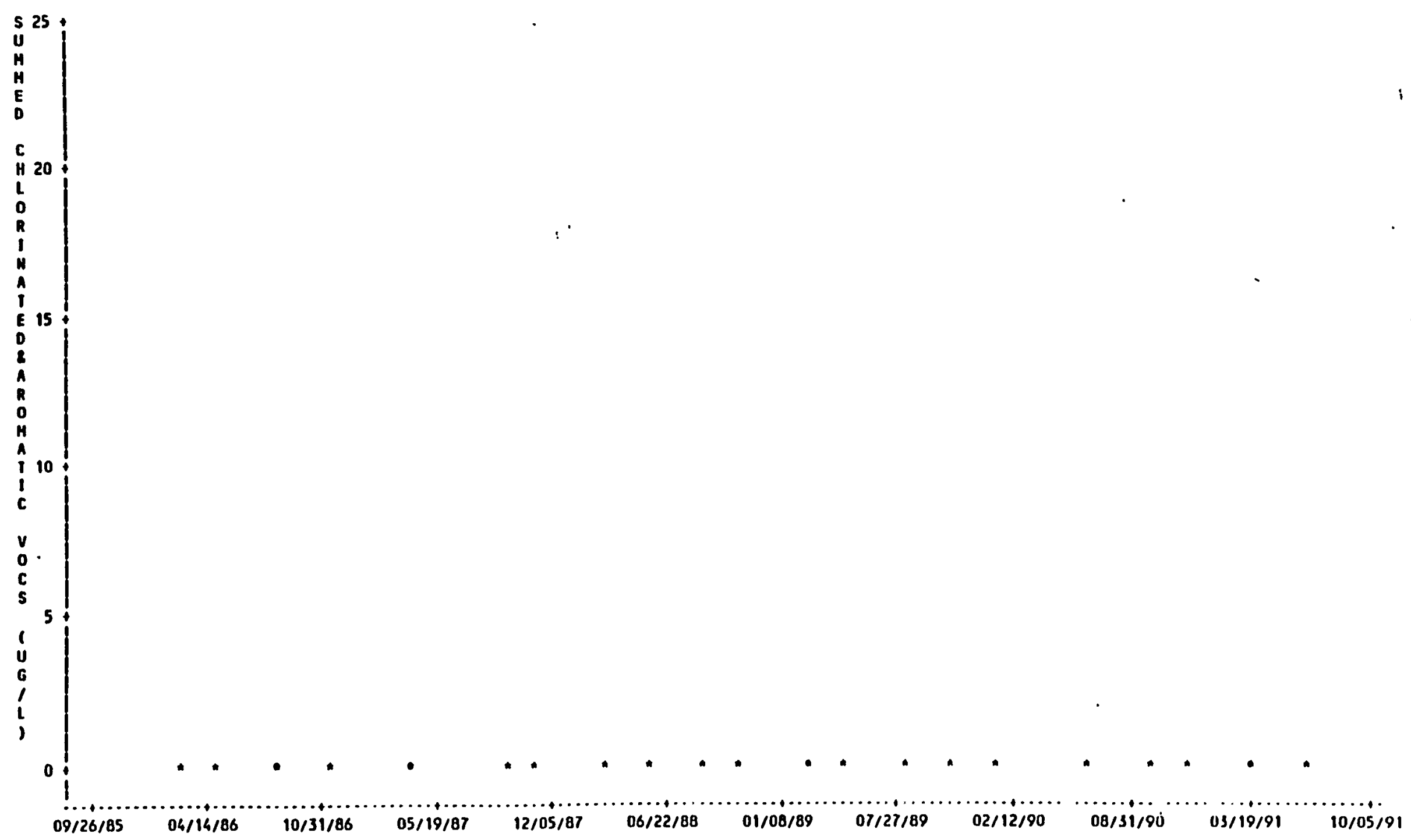

Figure 14b. Plot of summed chlorinated and aromatic VOCs for upgradient well GW-145 at Kerr Hollow Quarry. Each data point is the mean of four measurements. 


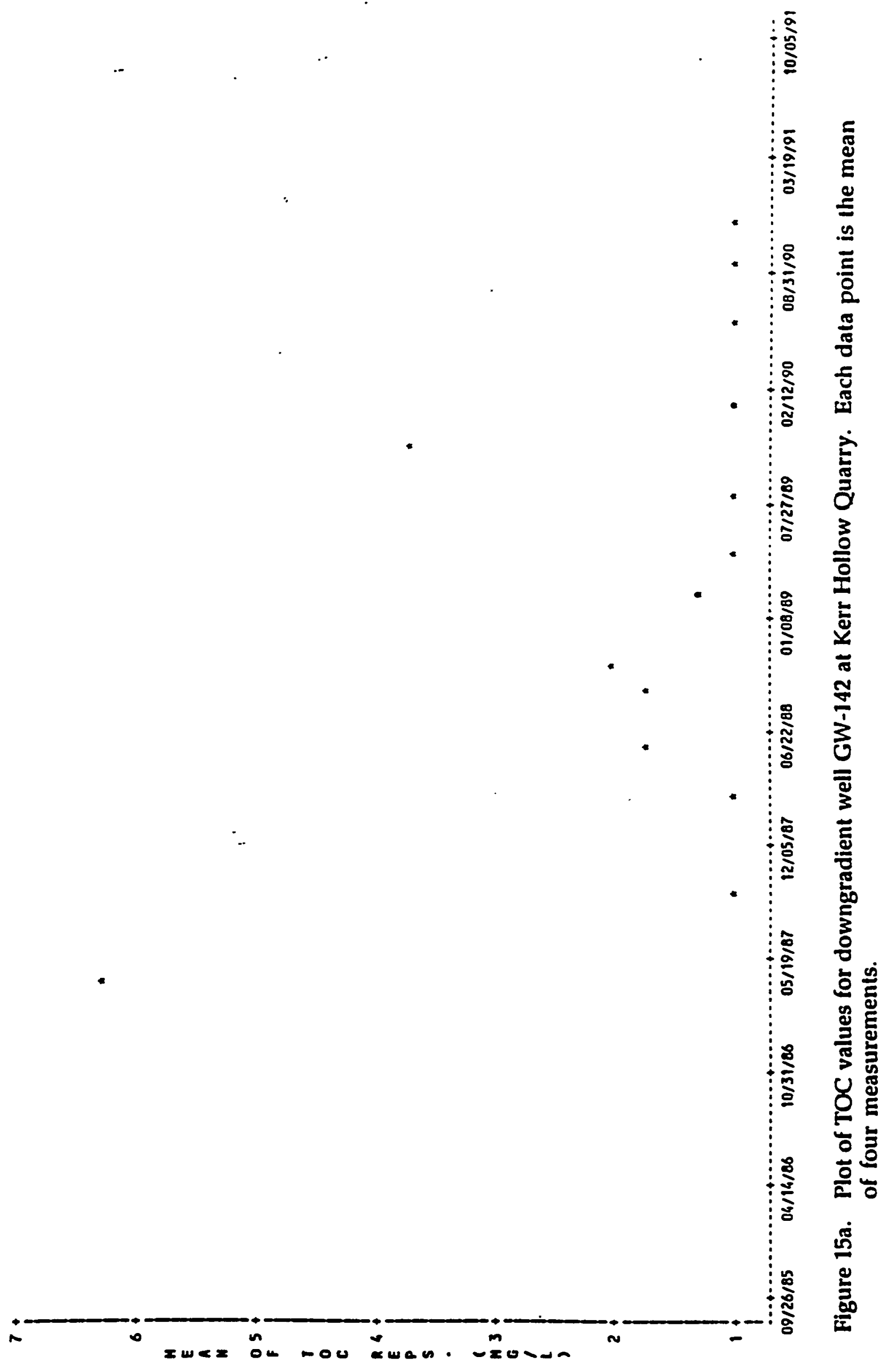




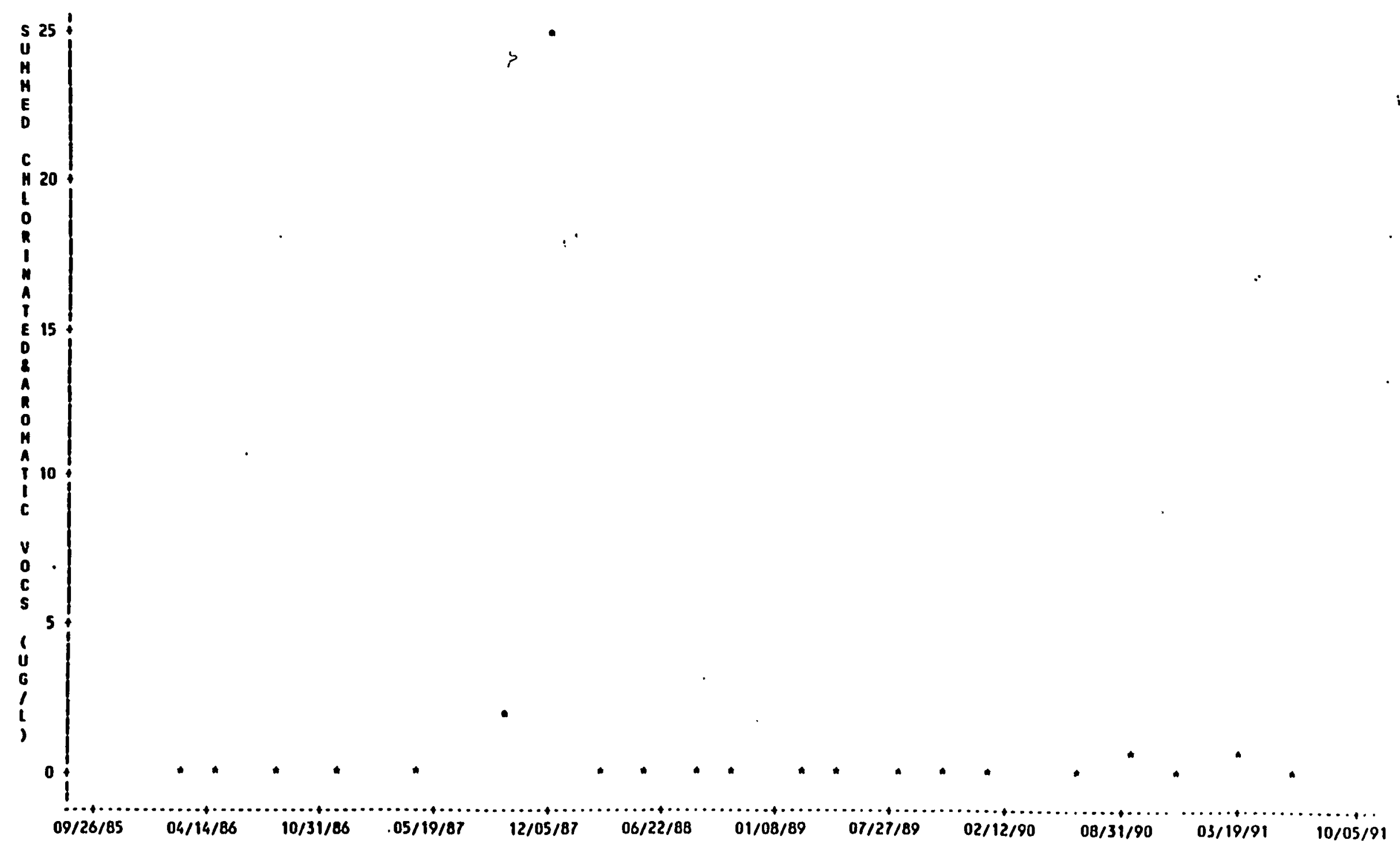

Figure 15b. Plot of summed chlorinated and aromatic VOCs for downgradient well GW-142 at Kerr Hollow Quarry. Each data point is the mean of four measurements. 


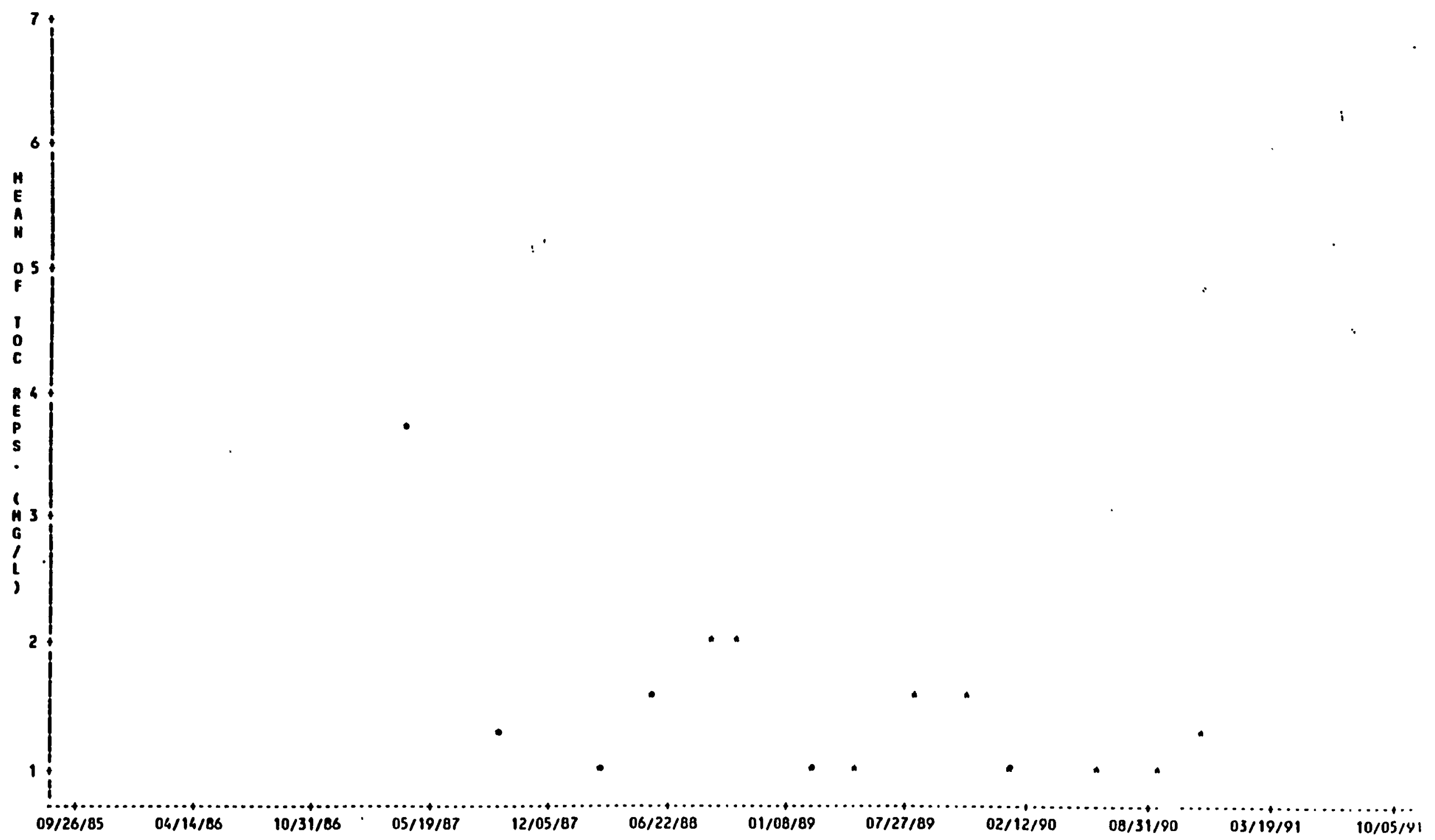

Figure 16a. Plot of TOC values for downgradient well GW-144 at Kerr Hollow Quarry. Each duta puil.. is the i..ean of four measurements. 


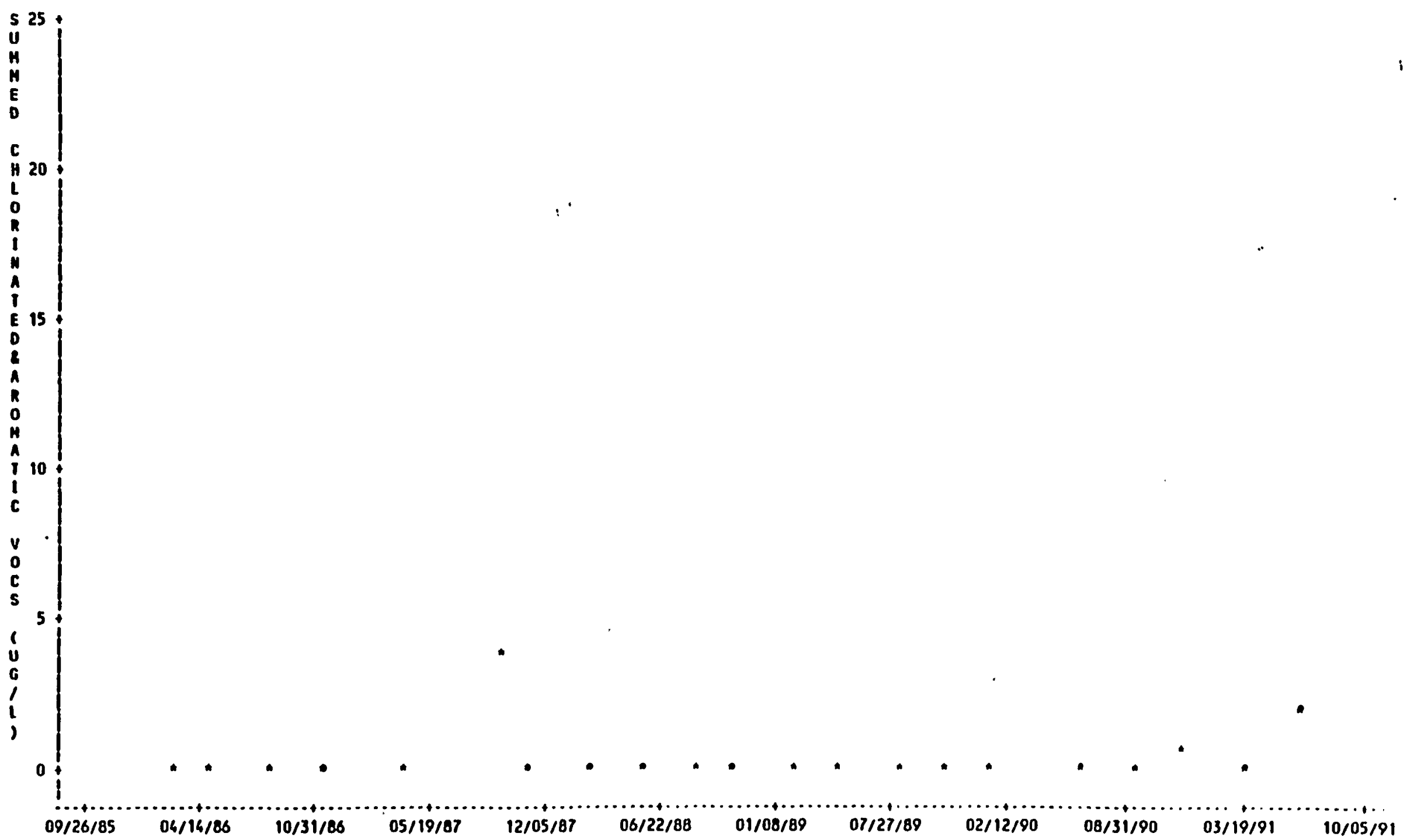

Figure 16b. Plot of summed chlorinated and aromatic VOCs for downgradient well GW-144 at Kerr Ilollow Quarry. Each data point is the mean of four measurements. 


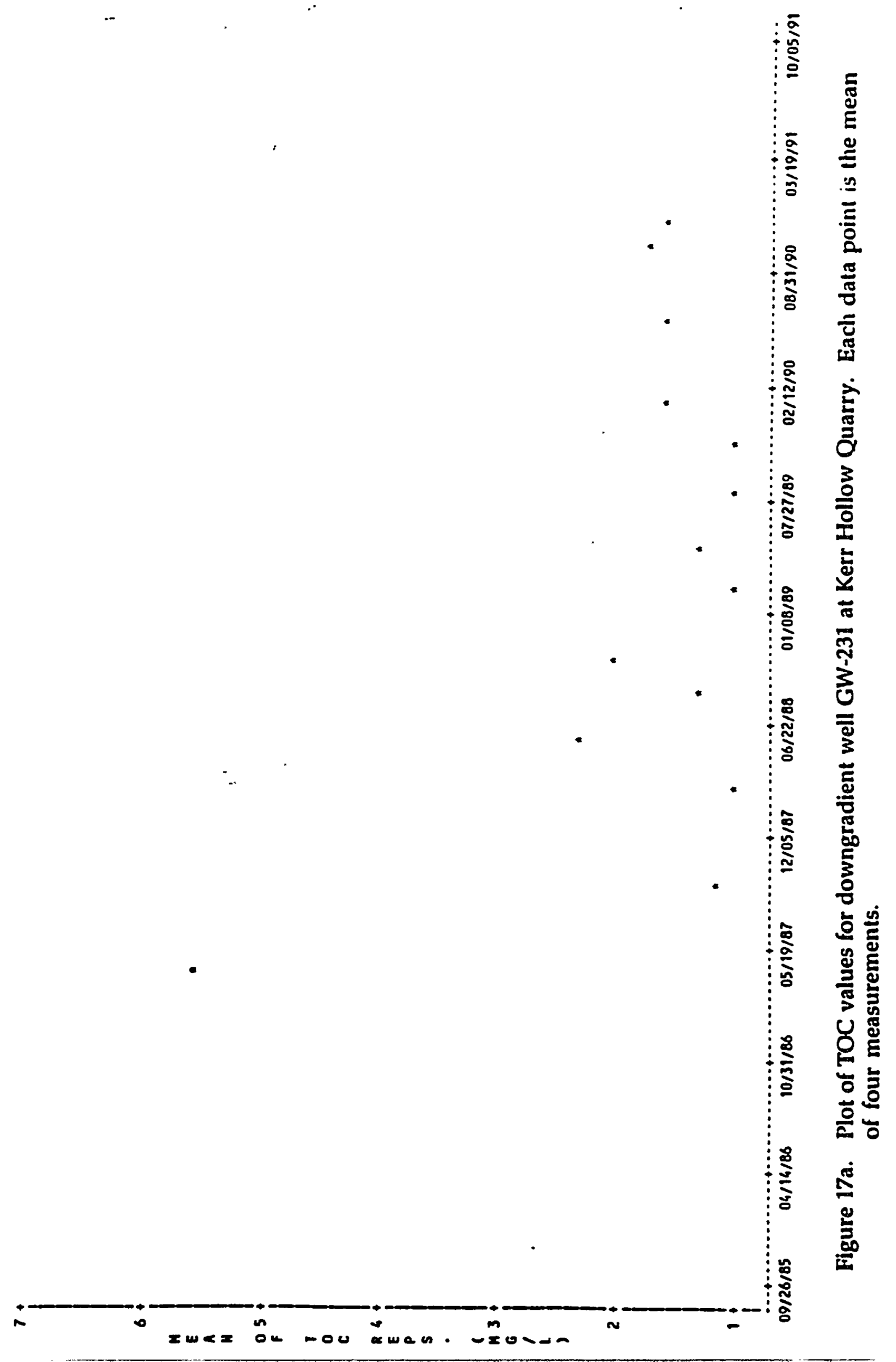




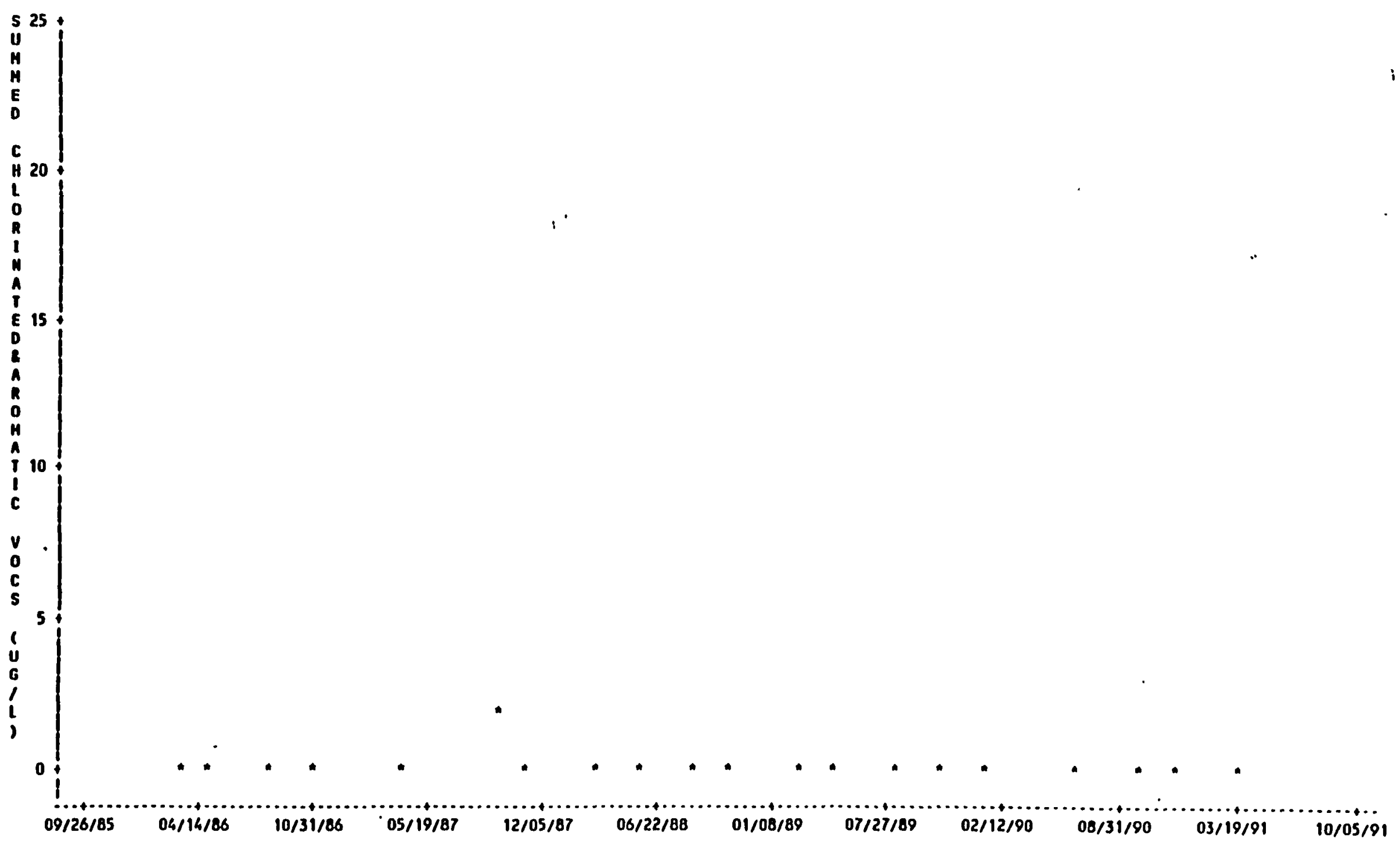

Figure 17b. Plot of summed chlorinated and aromatic VOCs for downgradient well GW-231 at Kerr Hollow Quarry. Each data point is the mean of four measurements. 


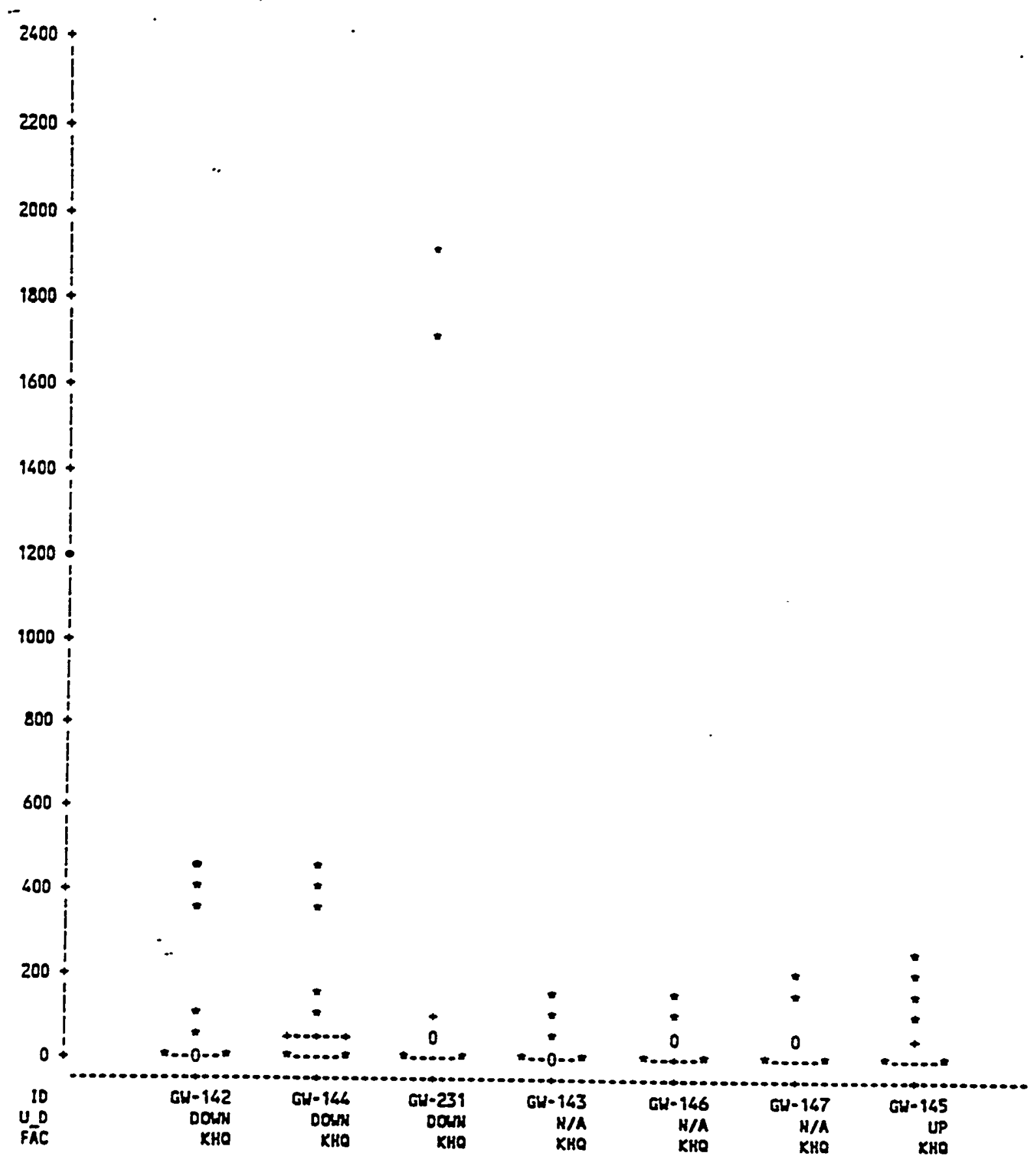

Figure 18. Statistical summary of TOX data for groundwater monitoring wells at the Kerr Hollow Quarry. Box outlines represent the 25th percentile (bottom of box), median (middle line terminated with asterisks), and 75th percentile (top of box) of the data set for a individual well. Mean is indicated by central plus sign ( + ) within the box. Central lines or tails extending above and below the box indicate the range of outliers within 1.5 interquartile ranges below the 25 th percentile and above the 75th percentile. Outliers beyond this range are illustrated by zero $(0)$ if they are within 3 interquartile ranges. More extreme outliers are illustrated by an asterisk (*). Because of scale, some boxes appear incomplete. 



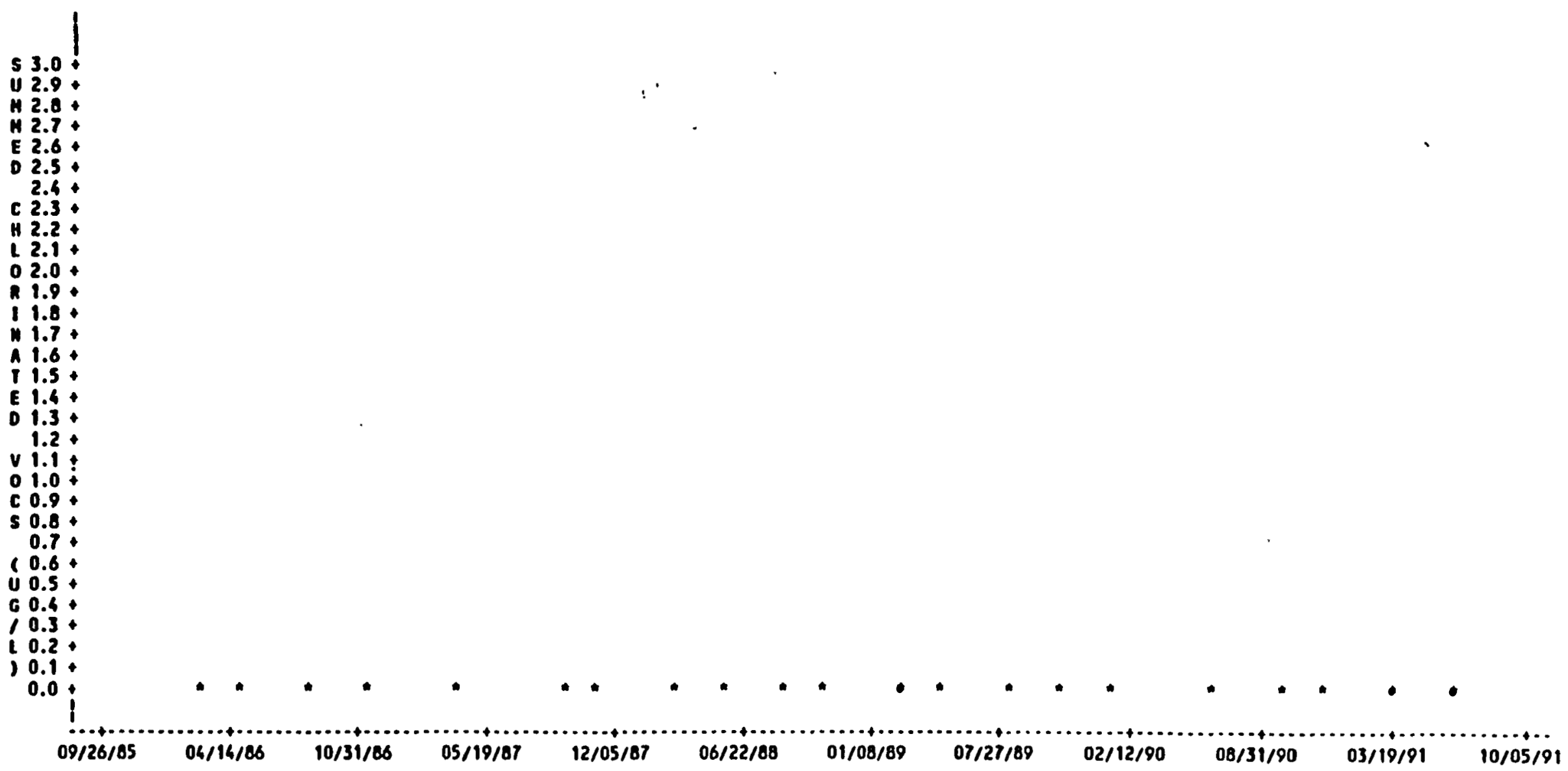

Figure 19b. Plot of summed chlorinated VOCs for upgradient well GW-145 at Kerr Hollow Quarry. Each data point is the mean of four measurements. 


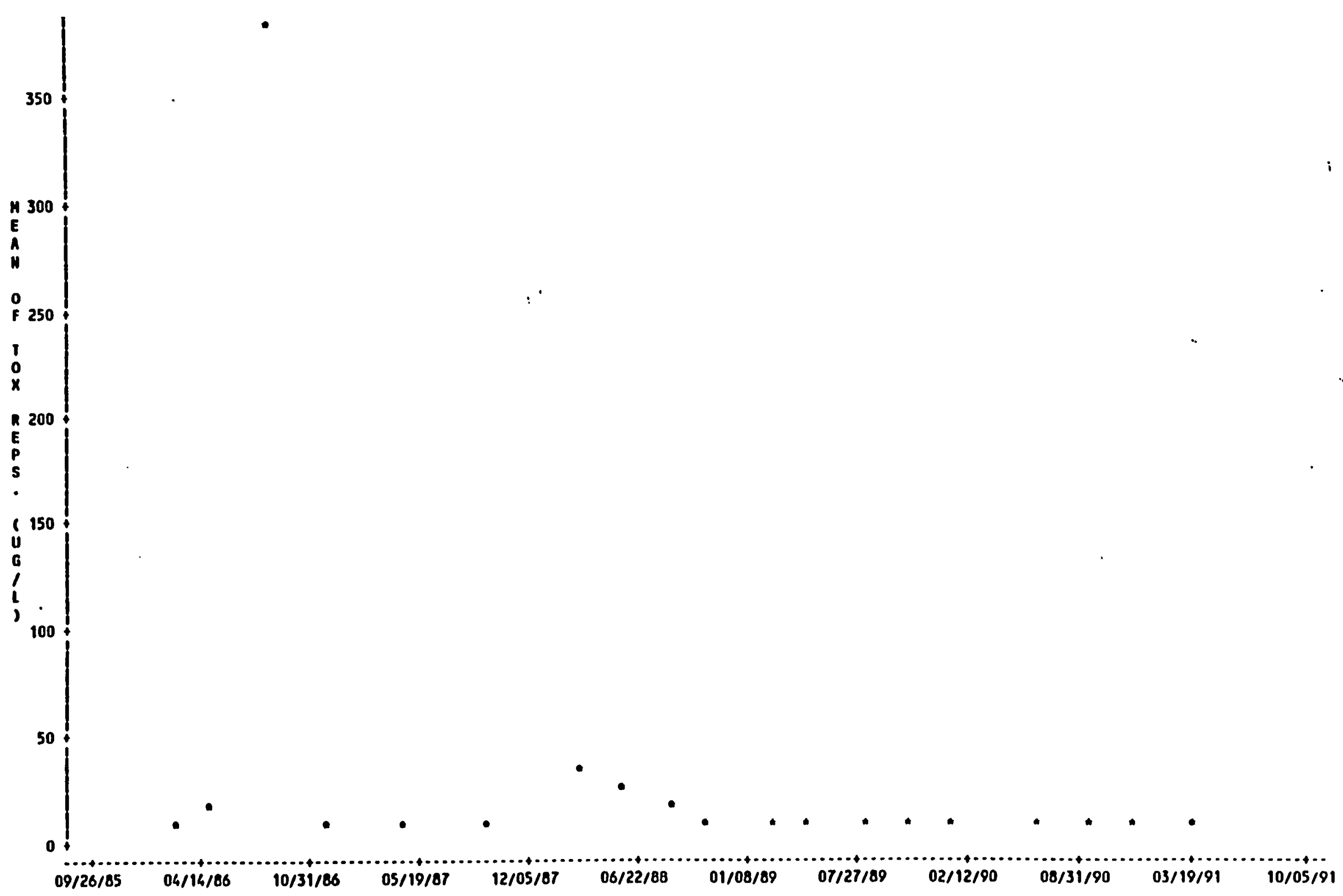

Figure 20a. Plot of TOX values for downgradient well GW-142 at Kerr Hollow Quarry. Each data point is the mean of four measurements. 


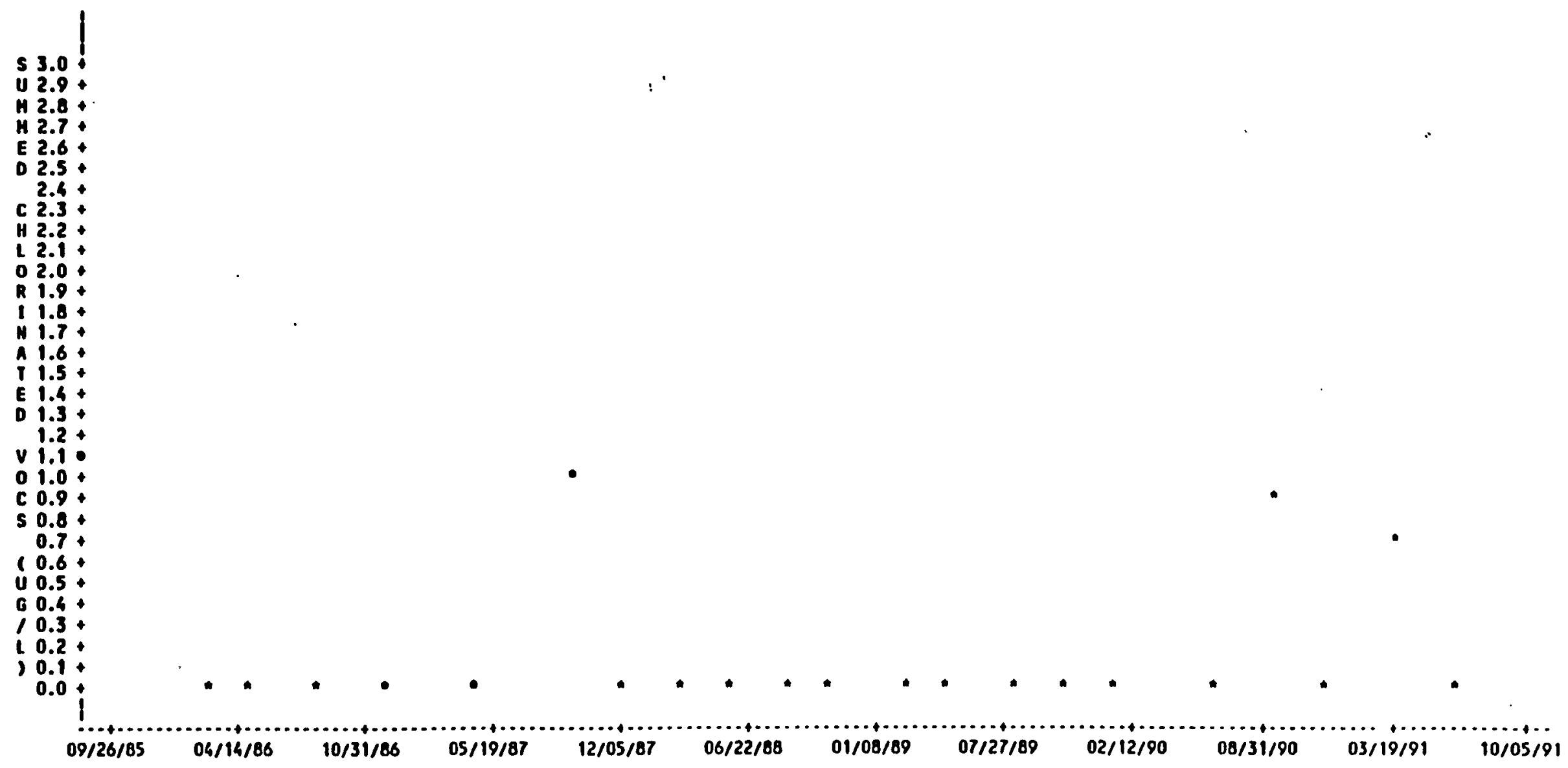

Figure 20b. Plot of summed chlorinated VOCs for downgradient well GW-142 at Kerr Hollow Quarry. Lach data point is the mean of four measurements.

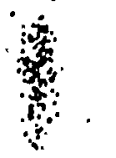




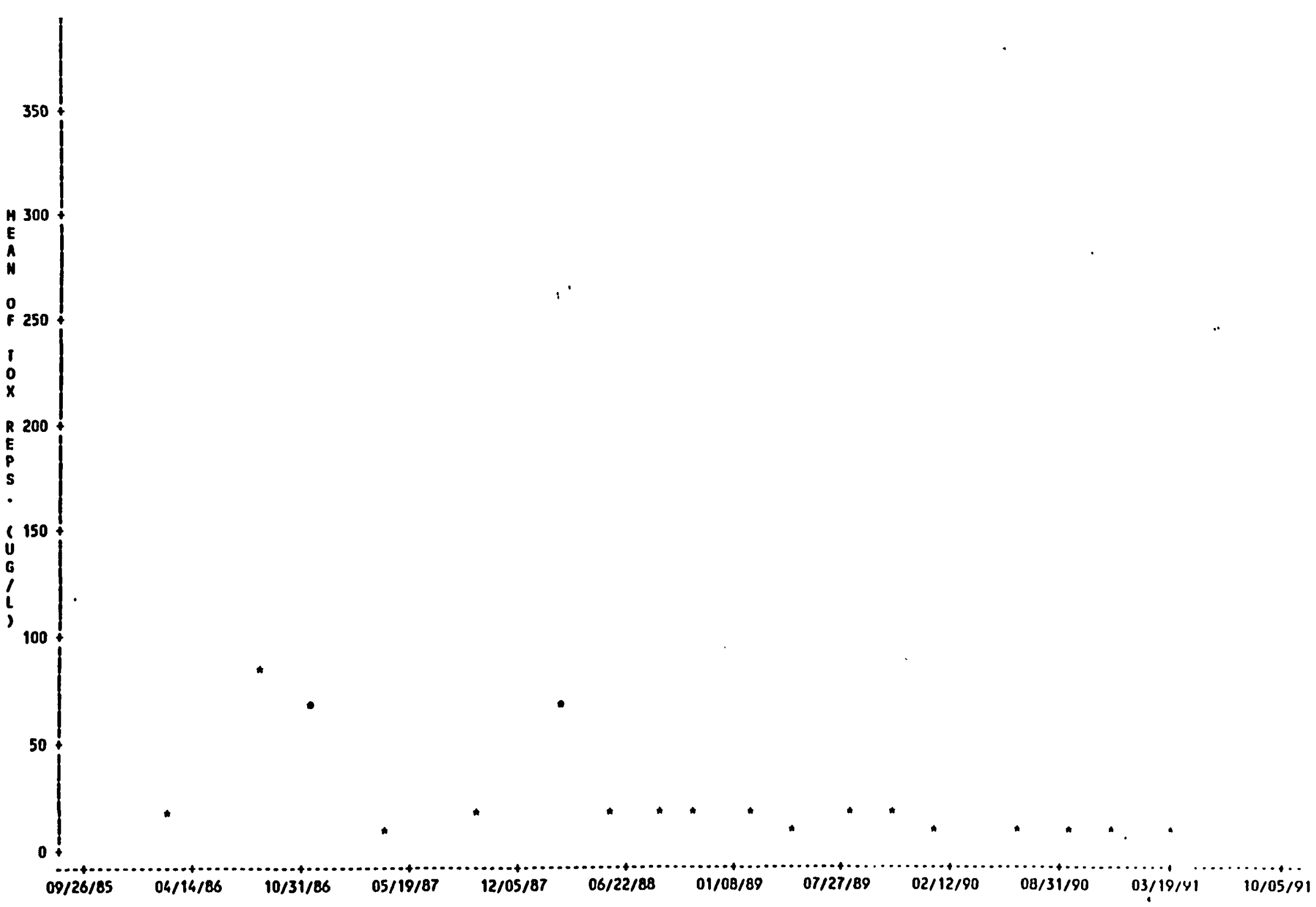

Figure 21a. Plot of TOX values for downgradient well GW-144 at Kerr Hollow Quarry. Each data point is the mean of four measurements. 
Słuauasnseau inoj jo ueaur ayı s! ju!̣od

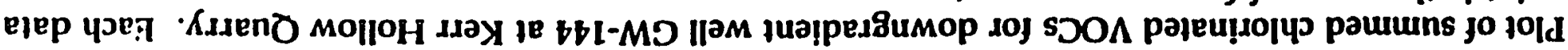

qIz aing!t

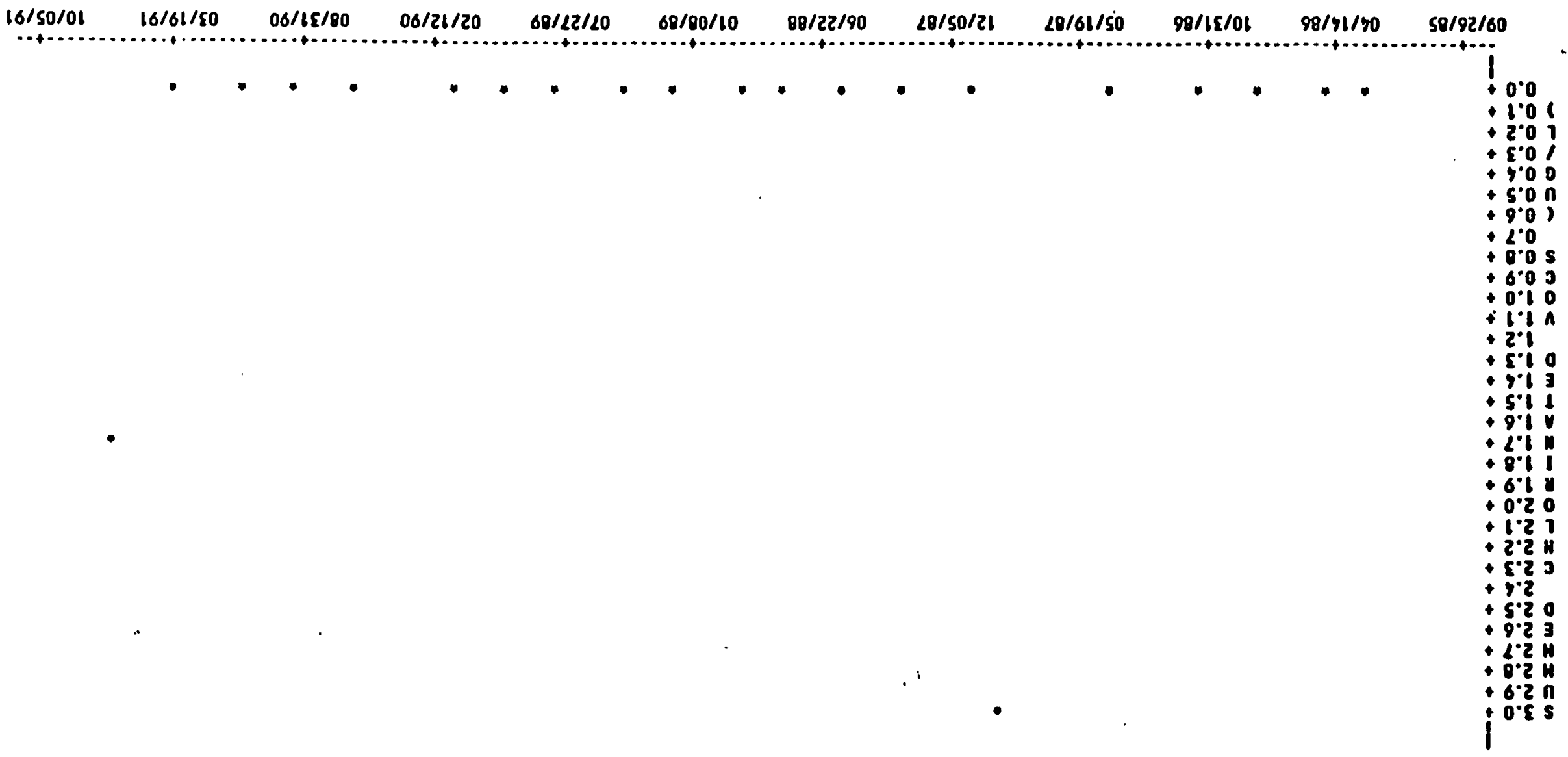




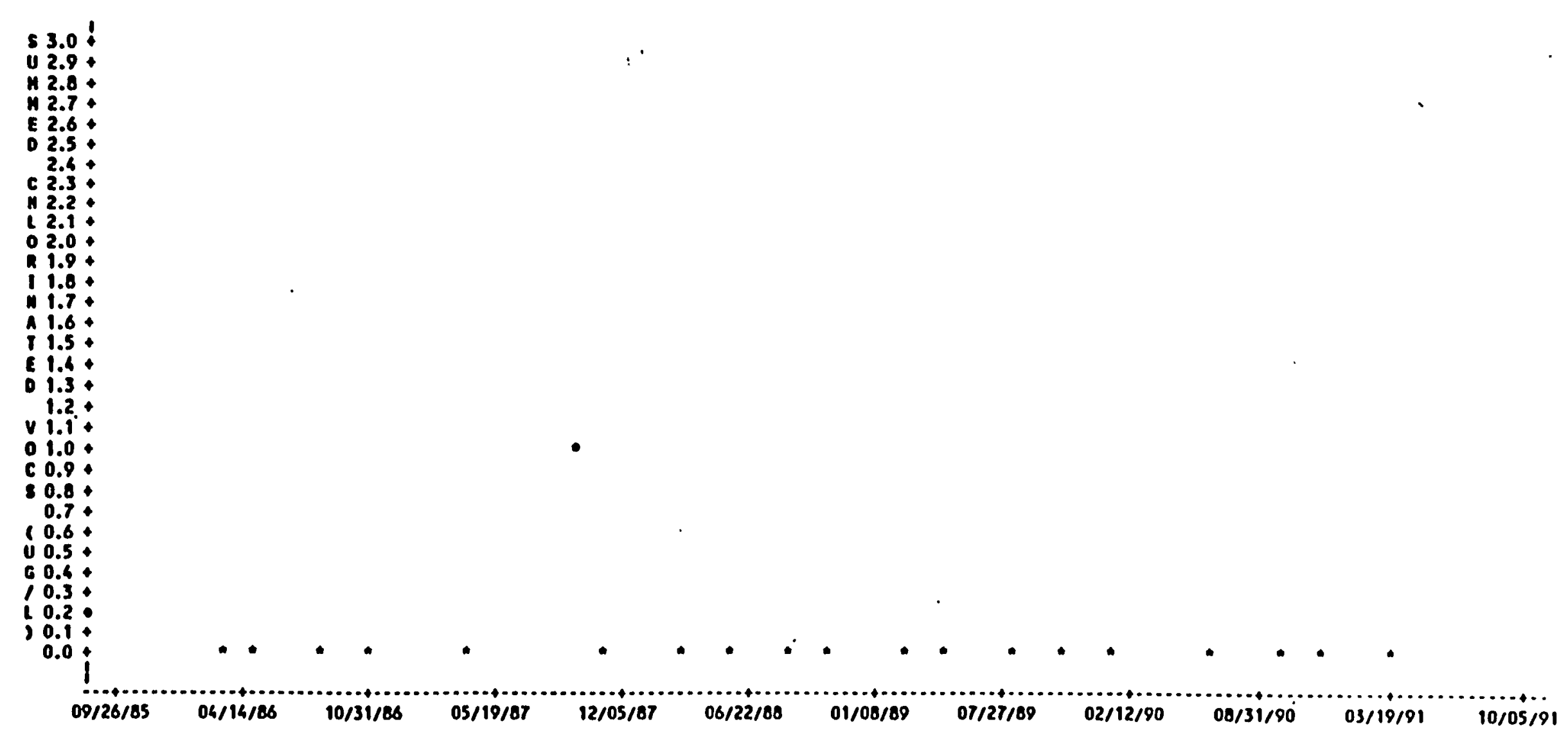

Figure 22b. Plot of summed chlorinated VOCs for downgradient well GW-231 at Kerr Hollow Quarry. Lach data point is the minn of fodit it aurionents.

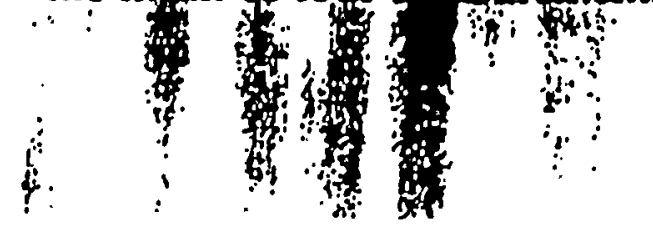




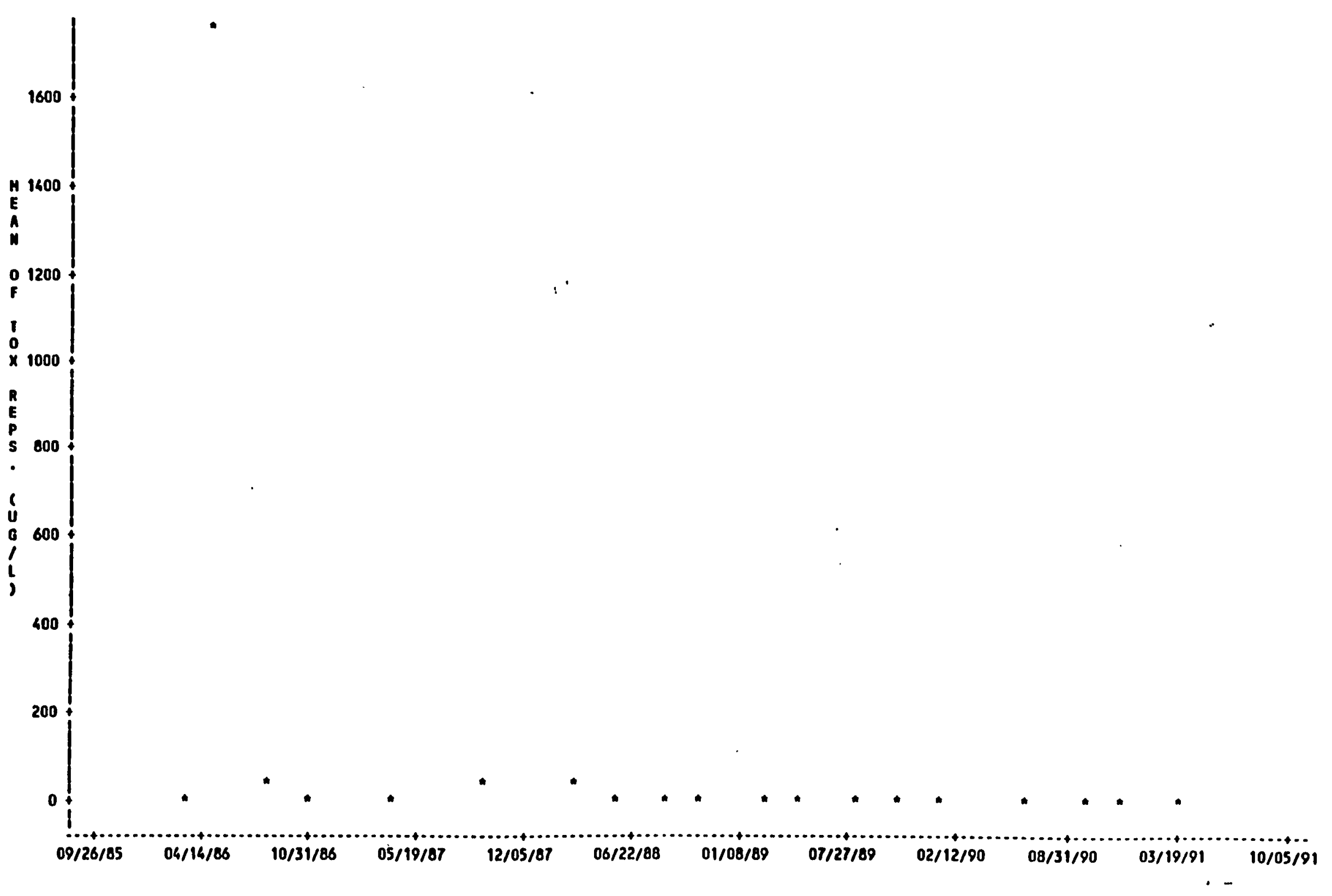

Figure 22a. Plot of TOX values for downgradient well GW-231 at Kerr Hollow Quarry. Each data point is the mean of four measurements. 


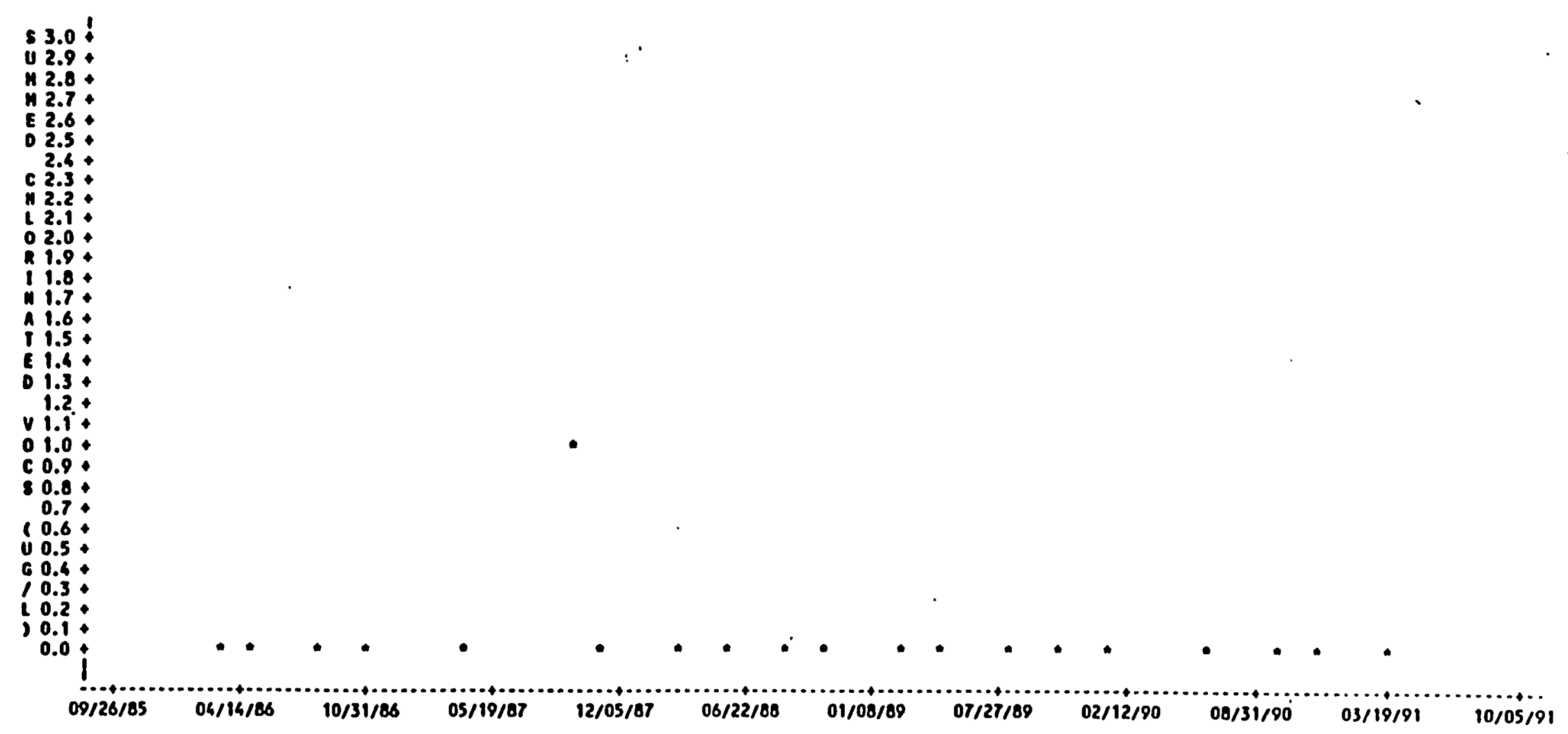

Figure 22b. Plot of summed chlorinated VOCs for downgradient well GW-231 at Kerr Hollow Quarry. Each data point is the minn of fotit is ariments.

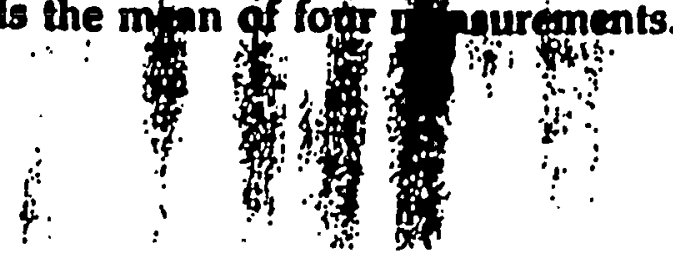


APPENDIX F

TRACE METAL EVALUATIONS AND TOLERANCE LIMIT DETERMINATIONS FOR GROUNDWATER AT KERR HOLLOW QUARRY 


\section{Trace Metal Evaluations and Tolerance Limit Determinations for Groundwater at Kerr Hollow Quarry}

Metal concentrations in groundwater from seven wells near Kerr Hollow Quarry have been measured approximately quarterly since January 1986. Three of these wells (GW-142, GW-147, and GW-231) are considered upgradient of the quarry and four are downgradient (GW-143, GW-144, GW-145, and GW-146). Statistical analyses were performed to determine whether trace metal concentrations in the upgradient wells differed statistically from concentrations observed in the downgradient wells and to determine a tolerance limit concentration that could be used as an indicator of contamination in samples taken in the future. Based on the known wastes disposed in the quarry, nine metals were chosen for analysis: barium, boron, cadmium, chromium, lead, mercury, nickel, strontium, and uranium. Total metals (unfiltered samples) were analyzed by atomic adsorption or inductively coupled plasma methods except for total uranium which was determined by a fluorometric technique. Statistical methods followed U.S. Environmental Protection Agency (EPA) Resource Conservation and Recovery Act (RCRA) guidance documents (EPA 1989; EPA 1992).

\section{Approach}

Concentrations of each analyte were plotted versus time (Figures F-1 through F-9). These plots were evaluated to determine whether the variation in concentration was random over time or displayed a trend. The plots were also examined for data outliers and obvious differences between wells. Based on this evaluation, some data were excluded from subsequent statistical tests. Barium and strontium concentrations in upgradient well GW-142 in 1986 were excluded from the analysis. The concentrations of barium and strontium in GW-142 approximately doubled between 1986 and 1988 (Figures F-1 and F-8, respectively). The concentrations from 1988 through 1994, however, do not appear to have a trend over time. Excluding the 1986 data for barium and strontium for GW-142 removes the temporal trend and is more representative of the most recent conditions in the well. Uranium concentrations from the last quarter of 1994 were excluded for all wells because the detection limit for this sampling round was an order of magnitude higher than for the previous data. Lead results for all wells were excluded for the 1988 and 1989 sampling rounds again because the detection limit for these results was much higher than for the other data. 
For cadmium, chromium, mercury, and nickel, which were detected in $<10 \%$ of the samples, concentrations greater than the detection limits did not appear to occur randomly over the sampling period but were concentrated in the period between 1990 and 1992 . This pattern of detected values suggests an unusual occurrence during that period of time. Trace metal contamination may have occurred in the groundwater during this period because of disturbances related to closure activities at the quarry. Because closure activities have ended and these trace metals were not detected during the most recent year of sampling, tolerance limits were not calculated. Further comparison between upgradient and downgradient wells was not conducted for these five analytes.

The upgradient and downgradient wells were statistically compared to determine if the concentrations of each of the remaining five analytes (barium, boron, lead, strontium, and uranium) were significantly different. A Shapiro-Wilk test was performed on the data from the upgradient and downgradient wells for each analyte to determine whether the distributions of the concentrations were normal or log-normal. In each case the distribution was significantly different (at the 0.05 probability level) from both normal and log-normal distributions indicating that comparisons should be made with nonparametric statistics (Table F-1). For analytes with $>50 \%$ of the concentrations above the detection limit (barium, boron, strontium, and uranium) the Wilcoxon Rank Sum test was used to determine if the upgradient concentrations were significantly different from the downgradient concentrations (at the $\mathbf{0 . 0 5}$ probability level). For lead, which had $<50 \%$ but $>10 \%$ of the results above the detection limit, a test of proportions was used to compare the upgradient and downgradient concentrations. The data were also plotted as cumulative frequency plots to allow a visual comparison of the upgradient and downgradient distributions (Figures F-10 to F-14). For barium the concentrations in the upgradient wells were significantly higher than the concentrations in the downgradient wells. Therefore, only the upgradient wells were used for determining the tolerance limit. For strontium and boron, the concentrations in the upgradient wells were significantly lower than the concentrations in the downgradient wells. Therefore, only the upgradient wells were used for determining the tolerance limits. For lead and uranium, the concentrations in the upgradient wells were not significantly different from the downgradient wells and therefore all wells were used to determine the tolerance limit. 
Because the distribution of the data was neither normal nor log-normal, a nonparametric 95\% upper tolerance limit (UTL) was used for all the analytes. One is $95 \%$ confident that $95 \%$ of the concentrations should fall below the 95\% UTL. For analytes with between 59 and 89 results, the $95 \%$ UTL is the maximum observed result. For cadmium, chromium, mercury, and nickel, which had a low frequency of non-random detected values, the maximum contaminant level (MCL) specified in the Safe Drinking Water Act was used as the tolerance limit. The tolerance limits for each analyte are provided in Table F-2 and discussed individually below.

Barium. The concentrations of barium in well GW-142 during 1986 were excluded from the statistical analysis because the concentrations in 1986 appeared to be significantly lower than the remaining period of record (Figure F-1). Using only the later data better represents current conditions. The mean concentration of the upgradient wells was significantly greater than the downgradient wells at the $\mathbf{0 . 0 5}$ probability level using the Wilcoxon Rank Sum test. Therefore only the upgradient wells were used to determine the tolerance limit. The nonparametric 95\% UTL was $0.49 \mathrm{mg} / \mathrm{L}$.

Boron. Other than the 1986 data, the boron concentrations appear fairly stable over time (Figure F-2). One result from GW-142 in the second quarter of 1986 was much higher than all subsequent results and was excluded from consideration. The concentration in the upgradient wells was significantly lower than the downgradient wells at the 0.05 probability level using the Wilcoxon Rank Sum test. However, pairwise tests indicated significant differences in concentration between all wells except GW-142 and GW-147. Therefore, no UTL was calculated. Boron is not an Appendix VIII constituent and does not have an established MCL. Therefore, annual trending will be conducted rather than using a comparative concentration.

Cadmium. Less than $10 \%$ of all cadmium results were above the detection limit (Table F-1). All of the detected values occurred between the second quarter of 1990 and the second quarter of 1992 (Figure F-3) although the detection limit was lowered in 1990 from $0.003 \mathrm{mg} / \mathrm{L}$ to $0.002 \mathrm{mg} / \mathrm{L}$. Values presented in Figure F-3 for data prior to 1990 are detection limits rather than actual detections. Cadmium was not detected in any wells prior to 1990 . These elevated levels detected in groundwater were coincident with closure activities at the site which involved the removal of materials from the sediments in the quarry. Therefore, those detected values were 
not used to calculate a tolerance limit for future sampling. The MCL of $0.005 \mathrm{mg} / \mathrm{L}$ was chosen as the tolerance limit.

Chromium. Less than $10 \%$ of all chromium results were above the detection limit (Table F-1). All of the detected values occurred between the second quarter of 1990 and the second quarter of 1992 (Figure F-4). As noted for cadmium, the frequency of detected values does not appear to be random over time; therefore, the observed detected values were not used to calculate a tolerance limit for future sampling. The MCL of $0.1 \mathrm{mg} / \mathrm{L}$ was chosen as the tolerance limit.

Lead. Lead was detected in $\sim 25 \%$ of the samples (Table F-1). Most of the detected values appeared in the 1986 samples but it has been detected sporadically since then (Figure F-5). Data from 1988 and 1989 were rejected because the detection limit was $0.05 \mathrm{mg} / \mathrm{L}$, an order of magnitude above the detection limit for the other periods. A test of proportions was used to determine if the proportion of detected values was significantly larger for the downgradient than the upgradient samples. The test indicated that the proportion of detected values was not significantly different at the $\mathbf{0 . 0 5}$ probability level. The shape of the cumulative frequency plots of the sample groups was similar (Figure F-12). Results from both the upgradient and downgradient wells were therefore used to determine the tolerance limit. The non-parametric 95\% UTL was $0.035 \mathrm{mg} / \mathrm{L}$.

Mercury. Only three mercury results were above the detection limit and all of these were before 1992 (Table F-1 and Figure F-6). As noted for cadmium, the frequency of detected values does not appear to be random over time; therefore, the observed detected values were not be used to calculate a tolerance limit for future sampling. The MCL of $0.002 \mathrm{mg} / \mathrm{L}$ was chosen as the tolerance limit.

Nickel. About $10 \%$ of all nickel results were above the detection limit (Table F-1). Most of the detected values occurred between the second quarter of 1990 and the second quarter of 1992 (Figure F-7). As noted for cadmium, the frequency of detected values does not appear to be random over time; therefore, the observed detected values were not used to calculate a tolerance limit for future sampling. The MCL of $0.1 \mathrm{mg} / \mathrm{L}$ was chosen as the tolerance limit. 
Strontium. The concentrations of strontium in well GW-142 during 1986 were excluded from the statistical analysis because the concentrations in 1986 appeared to be lower than the remaining period of record (Figure F-8). Using only the later data better represent current conditions. The concentration in the upgradient wells was significantly lower than the downgradient wells at the 0.05 probability level using the Wilcoxon Rank Sum test. However, pairwise tests (Kruskal Wallis) indicated significant differences in concentration between all wells. Therefore, no UTL was calculated. Due to statistical variance between wells and the fact that strontium is not an Appendix VIII constituent and does not have an established MCL, annual trending of the data will be conducted.

Uranium. Data from the fourth quarter of 1994 were rejected for the statistical analyses because the detection limit was an order of magnitude higher than during the other sampling periods (Figure F-9). The concentration of the remaining upgradient wells was not significantly different from the downgradient wells at the $\mathbf{0 . 0 5}$ probability level using the Wilcoxon Rank Sum test. Therefore, data from both the upgradient and downgradient wells were used to calculate the tolerance limit. The non-parametric $95 \%$ UTL was $0.019 \mathrm{mg} / \mathrm{L}$.

\section{References:}

U.S. EPA, 1989. Statistical Analysis of Ground-water Monitoring Data at RCRA Facilities, Interim Final Guidance. Office of Solid Waste, Waste Management Division, U.S.EPA, Washington, D.C., February 1989. PB89-151047.

U.S. EPA, 1992. Addendum to the Interim Final Guidance on the Statistical Analysis of Groundwater Monitoring Data at RCRA Facilities. Office of Solid Waste, Waste Management Division, U.S.EPA, Washington, D.C., February. 
Table F-1. Summary statistics for indicator parameters

\begin{tabular}{|c|c|c|c|c|c|c|c|c|}
\hline Analyte & Group & $\begin{array}{l}\text { Detects/ } \\
\text { Samples }\end{array}$ & \%Detects & Mean & $\begin{array}{l}\text { Minimum } \\
\text { Detect }\end{array}$ & $\begin{array}{c}\text { Maximum } \\
\text { Detect }\end{array}$ & MCL & Distribution \\
\hline Barium & downgradient & $104 / 104$ & 100 & 0.0716 & 0.0024 & 0.54 & 2. & $\mathrm{X}$ \\
\hline Barium & upgradient & $73 / 73$ & 100 & 0.195 & 0.031 & 0.49 & 2. & $\mathrm{x}$ \\
\hline Boron & downgradient & $104 / 104$ & 100 & 0.396 & 0.016 & 1.10 & NA & $\mathrm{x}$ \\
\hline Boron & upgradient & $73 / 76$ & 96 & 0.0478 & 0.0062 & 0.17 & NA & $\mathrm{x}$ \\
\hline Cadmium & downgradient & $1 / 104$ & 0.962 & 0.00115 & 0.0023 & 0.0023 & 0.005 & $\mathrm{X}$ \\
\hline \multirow{12}{*}{ a } & upgradient & $3 / 78$ & 3.85 & 0.0012 & 0.0023 & 0.003 & 0.005 & $\mathrm{x}$ \\
\hline & downgradient & $5 / 104$ & 4.81 & 0.00566 & 0.011 & 0.025 & 0.1 & $\mathrm{x}$ \\
\hline & upgradient & $3 / 78$ & 3.85 & 0.00565 & 0.011 & 0.011 & 0.1 & D \\
\hline & downgradient & $23 / 92$ & 25 & 0.0065 & 0.004 & 0.128 & NA & $\mathrm{x}$ \\
\hline & upgradient & $16 / 68$ & 23.5 & 0.00514 & 0.004 & 0.11 & NA & $\mathrm{x}$ \\
\hline & downgradient & $2 / 92$ & 2.17 & 0.000103 & 0.00024 & 0.00025 & 0.002 & D \\
\hline & upgradient & $1 / 68$ & 1.47 & 0.000102 & 0.0002 & 0.0002 & 0.002 & D \\
\hline & downgradient & $11 / 104$ & 10.6 & 0.00657 & 0.01 & 0.051 & 0.1 & $\mathrm{x}$ \\
\hline & upgradient & $5 / 77$ & 6.49 & 0.00838 & 0.01 & 0.19 & 0.1 & $\mathrm{x}$ \\
\hline & downgradient & $104 / 104$ & 100 & 4.12 & 0.078 & 18.0 & NA & $\mathrm{x}$ \\
\hline & upgradient & $73 / 73$ & 100 & 0.576 & 0.018 & 3.6 & NA & $\mathrm{x}$ \\
\hline & downgradient & $88 / 103$ & 85.4 & 0.00474 & 0.001 & 0.02 & 0.02 & $\mathrm{x}$ \\
\hline Uranium & upgradient & $54 / 77$ & 70.1 & 0.00397 & 0.001 & 0.032 & 0.02 & $\mathrm{x}$ \\
\hline
\end{tabular}

Concentrations in $\mathrm{mg} / \mathrm{L}$.

NA - Not Applicable.

Results less than the detection limit were set to $1 / 2$ the reported detection limit.

Dist. Codes: $\mathrm{L}$ - distribution most similar to lognormal.

$\mathrm{N}$ - distribution most similar to normal.

$\mathrm{X}$ - distribution significantly different from normal and lognormal.

D - distribution not determined because fewer than 5 detected values. 
Table F-2. Tolerance limits for selected indicator parameters

\begin{tabular}{|c|c|c|c|c|}
\hline \multirow[t]{2}{*}{ Analyte } & \multicolumn{2}{|c|}{ Up- vs. Downgradient Comparison } & \multirow{2}{*}{$\begin{array}{l}\text { Tolerance Limit } \\
\text { (mg/L) }\end{array}$} & \multirow[t]{2}{*}{ Tolerance Limit Source } \\
\hline & Test & Result & & \\
\hline Barium & Wilcoxon & Up $>$ Down & 0.49 & Non-Parametric 95\% UTL \\
\hline Cadmium & NA & NA & 0.005 & MCL \\
\hline Chromium & NA & NA & 0.1 & MCL \\
\hline Lead & Proportions & Not Different & 0.035 & Non-parametric 95\% UTL \\
\hline Mercury & NA & NA & 0.002 & MCL \\
\hline Nickel & NA & NA & 0.1 & MCL \\
\hline Uranium & Wilcoxon & Down $>$ Up & 0.019 & Non-Parametric 95\% UTL \\
\hline
\end{tabular}

NA - Not Applicable 
Kerr Hollow Groundwater Data 1986-1994

Concentrations Over Time

Analyte (units) $=$ BARIUM $(M G / L)$

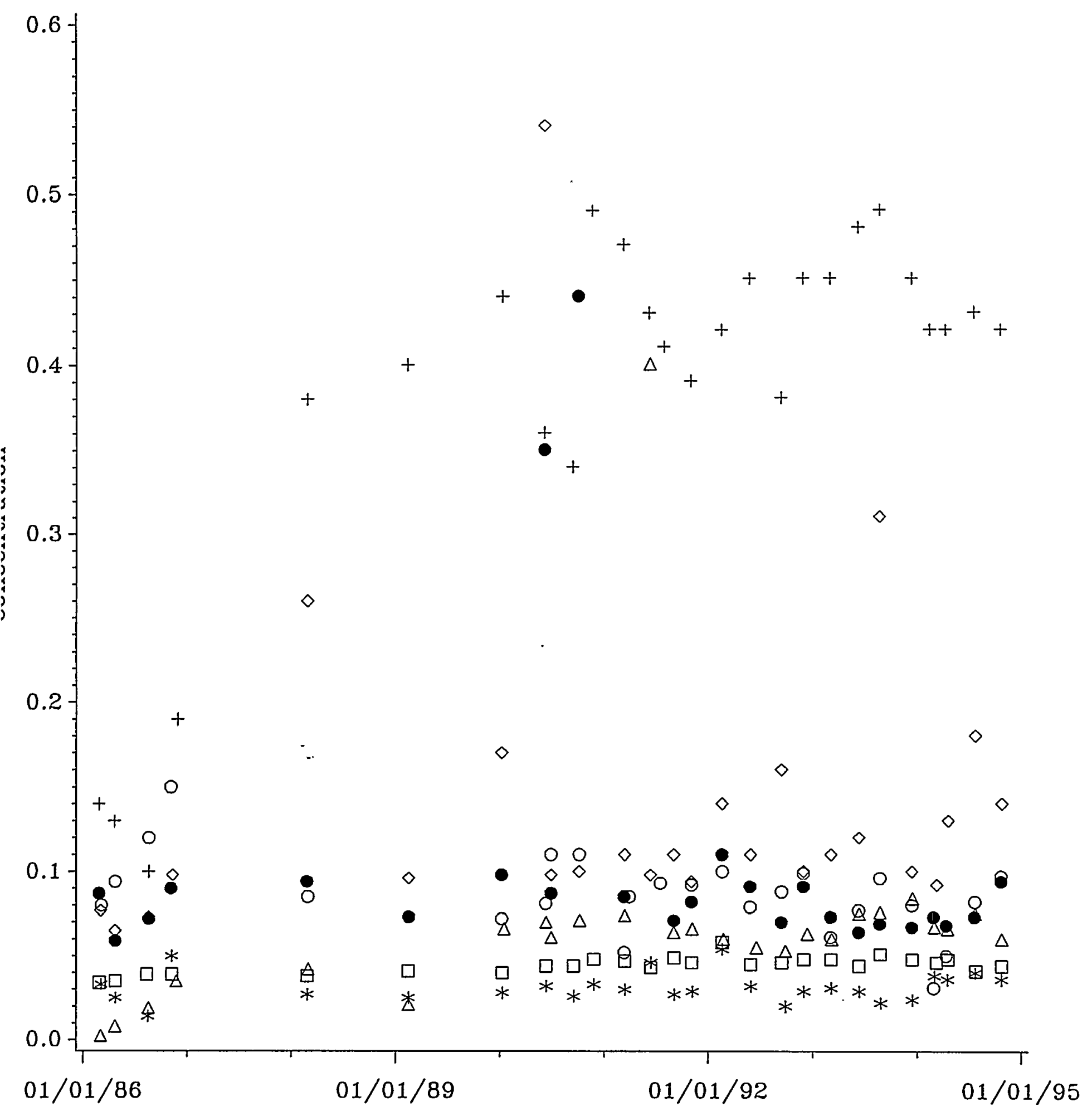

Date Collected

Well $+++\mathrm{GW}-142 * * *$ GW-143 $\square \square \square \mathrm{GW}-144 \quad \diamond \diamond \diamond \mathrm{GW}-145$

$\Delta \Delta \Delta \mathrm{GW}-146 \quad \bullet \bullet \bullet \mathrm{GW}-147 \quad \circ \circ \circ \mathrm{GW}-231$

Figure F-1. Barium concentrations over time.

nerated by program plot2 on $14 \mathrm{JUN} 95$ at $15: 46$ using datasets $\mathrm{y} 12 \mathrm{gw} \_\mathrm{mf}$ soilbg01 mndnew02 
Kerr Hollow Groundwater Data 1986-1994

Concentrations Over Time

Analyte (units) $=$ BORON $(\mathrm{MG} / \mathrm{L})$

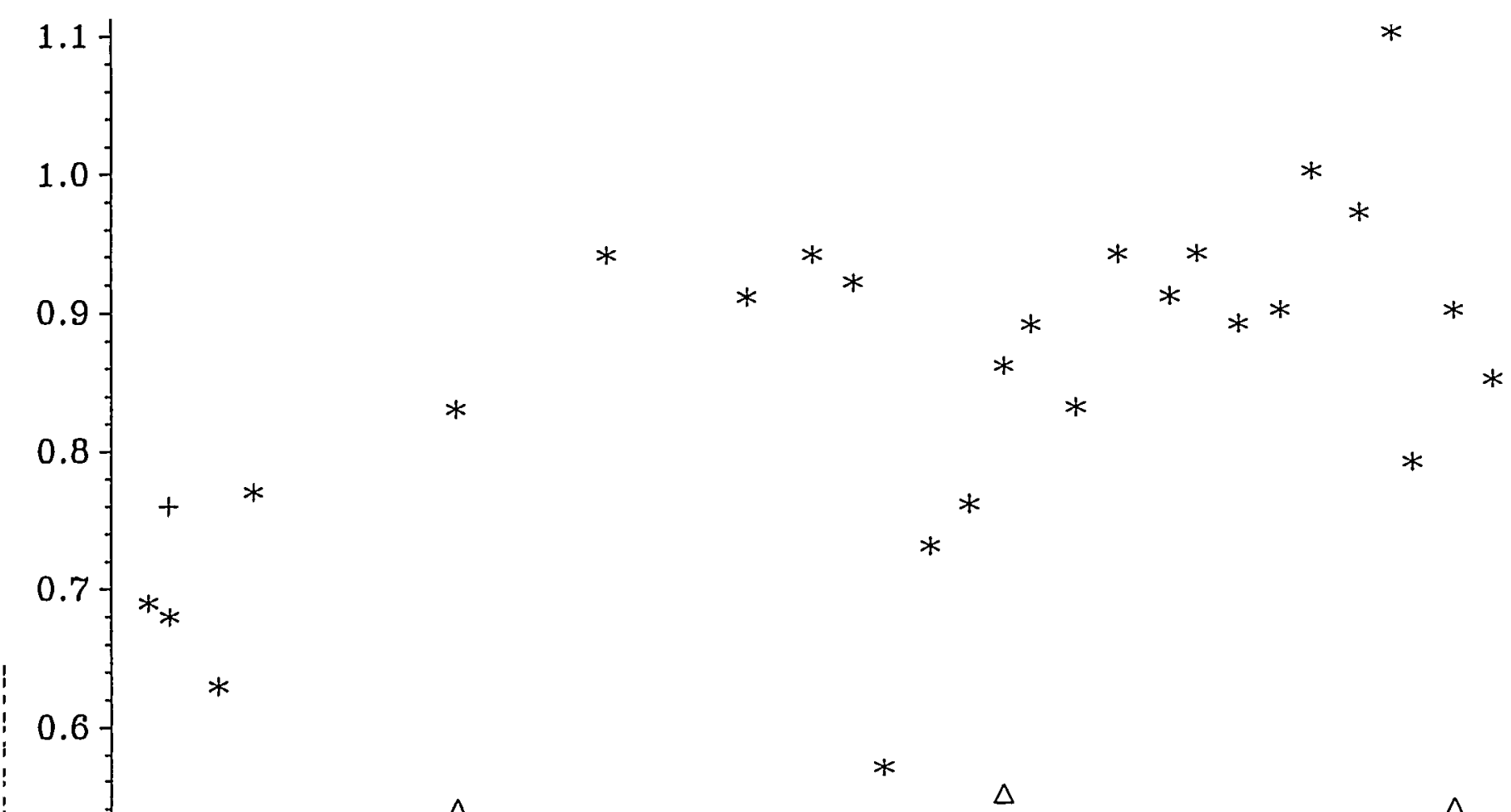

$\Delta$

$\Delta \Delta_{\Delta}^{\Delta} \Delta{ }_{\Delta}^{\Delta}{ }_{\Delta}^{\Delta} \Delta \Delta^{\Delta}{ }^{\Delta}{ }_{\Delta}^{\Delta} \Delta$

$\Delta \Delta_{\Delta} \Delta{ }_{\Delta}^{\Delta}{ }_{\Delta}^{\Delta} \Delta \Delta^{\Delta}{ }_{\Delta}^{\Delta} \Delta \Delta$

$0.4 \Delta \Delta$

$\Delta \quad \Delta$

$0.3 . \Delta \Delta$

$\diamond \quad \diamond \diamond \diamond \diamond \diamond \diamond \diamond \diamond \diamond \diamond \diamond \diamond \diamond \diamond \diamond$

$0.0-$

$\diamond \diamond$

$$
01 / 01 / 86
$$

01/01/89

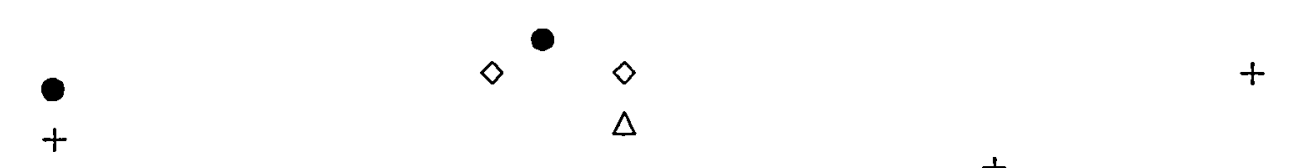

Well

$$
\begin{aligned}
& +++\mathrm{GW}-142 \\
& \Delta \Delta \Delta \mathrm{GW}-146
\end{aligned}
$$$$
\text { *** GW-143 }
$$$$
\text { - } \bullet \mathrm{GW}-147
$$

$\square \square \square \mathrm{GW}-144$

$\circ \circ \bigcirc \mathrm{GW}-231$

Figure F-2. Boron concentrations over time.

nerated by program plot2 on 14JUN95 at 15:46 using datasets y12gw_mf soilbg01 mndnew02 
Kerr Hollow Groundwater Data 1986-1994

Concentrations Over Time

Analyte (units) $=$ CADMIUM $(M G / L)$

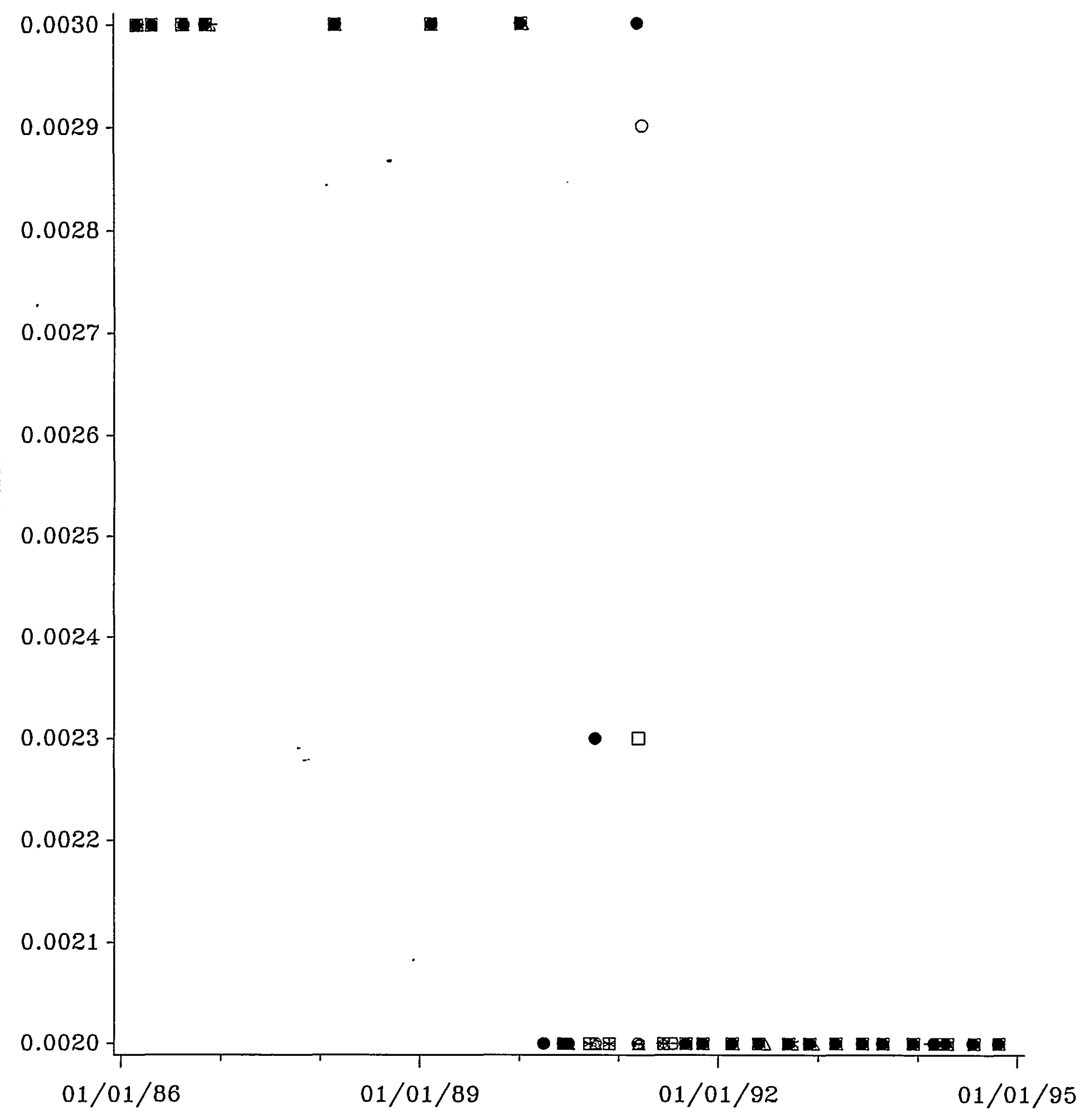

Date Collected

Well $+++\mathrm{GW}-142 * * *$ GW-143 ㅁ $\mathrm{GW}-144 \quad \diamond \diamond \diamond \mathrm{GW}-145$

$\Delta \Delta \Delta \mathrm{GW}-146 \bullet \bullet \bullet \mathrm{GW}-147 \quad \circ \circ \circ \mathrm{GW}-231$

Figure F-3. Cadmium concentrations over time.

nerated by program plot2 on $14 \mathrm{JUN} 95$ at 15:46 using datasets y12gw_mf soilbg01 mndnew02

F-10 
Kerr Hollow Groundwater Data 1986-1994

Concentrations Over Time

Analyte (units) $=$ CHROMIUM $(\mathrm{MG} / \mathrm{L})$

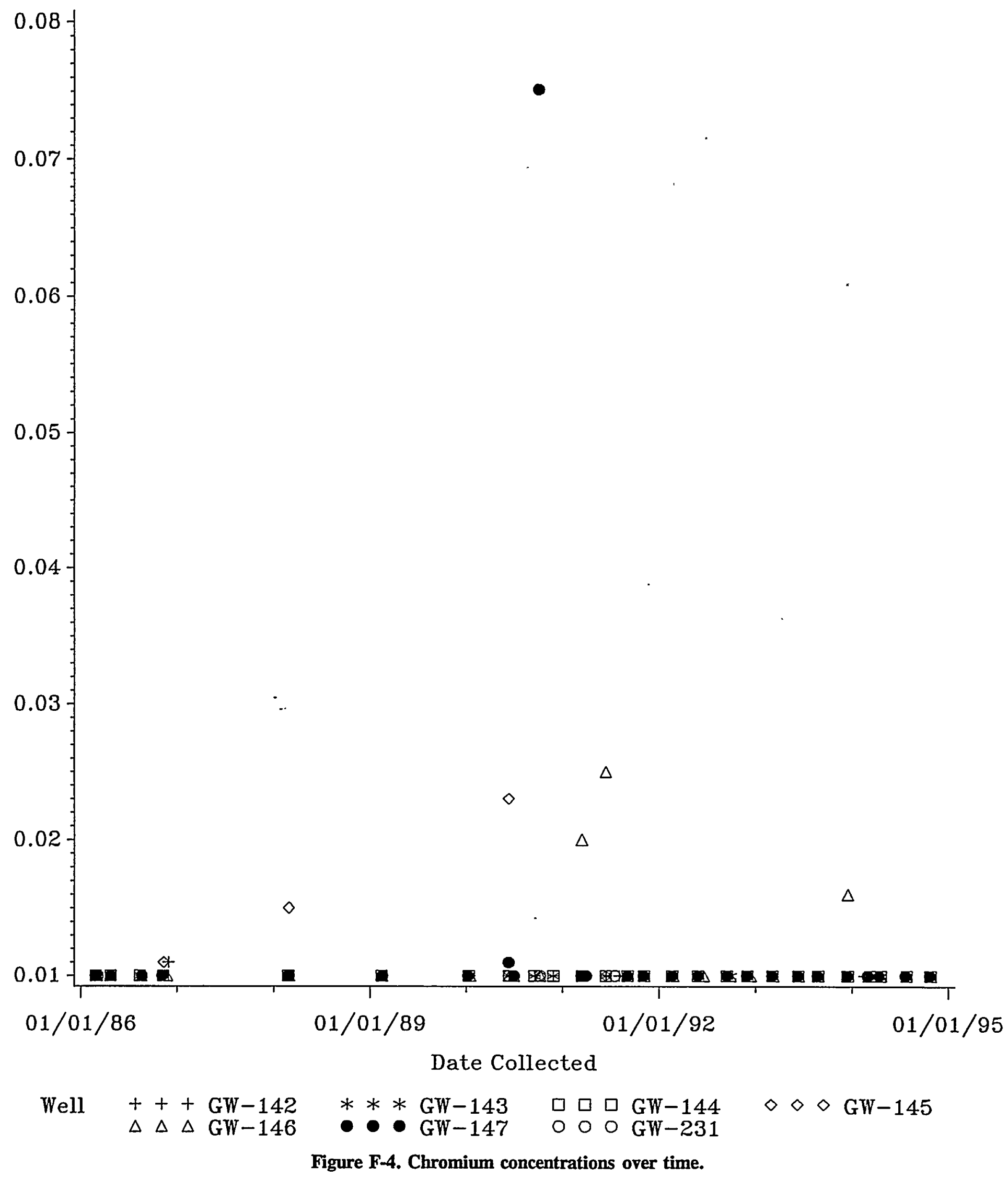

nerated by program plot2 on 14JUN95 at 15:46 using datasets y12gw_mf soilbg01 mndnew02 
Kerr Hollow Groundwater Data 1986-1994

Concentrations Over Time

Analyte (units) $=\mathrm{LEAD}(\mathrm{MG} / \mathrm{L})$

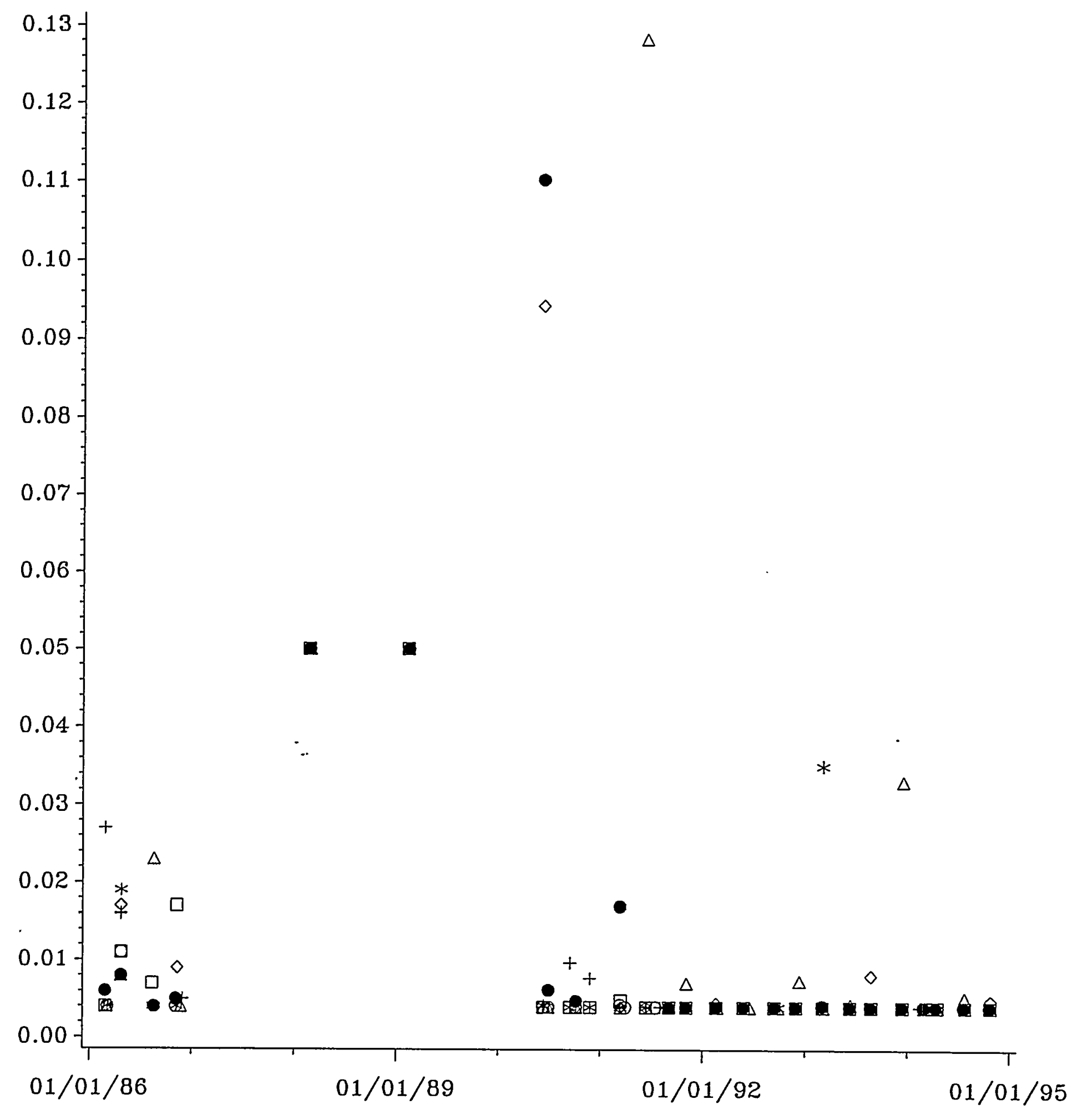

Date Collected

Well +++ GW-142 *** GW-143 ㅁㅁ GW-144 $\diamond \diamond \diamond \mathrm{GW}-145$

$\Delta \Delta \Delta \mathrm{GW}-146 \quad \bullet \bullet \mathrm{GW}-147 \quad 0$ ○ $0 \mathrm{GW}-231$

Figure F-5. Lead concentrations over time.

nerated by program plot2 on $14 \mathrm{JUN} 95$ at 15:46 using datasets y12gw_mf soilbg01 mndnew02 F-12 
Kerr Hollow Groundwater Data 1986-1994

Concentrations Over Time

Analyte (units) $=$ MERCURY $(\mathrm{MG} / \mathrm{L})$

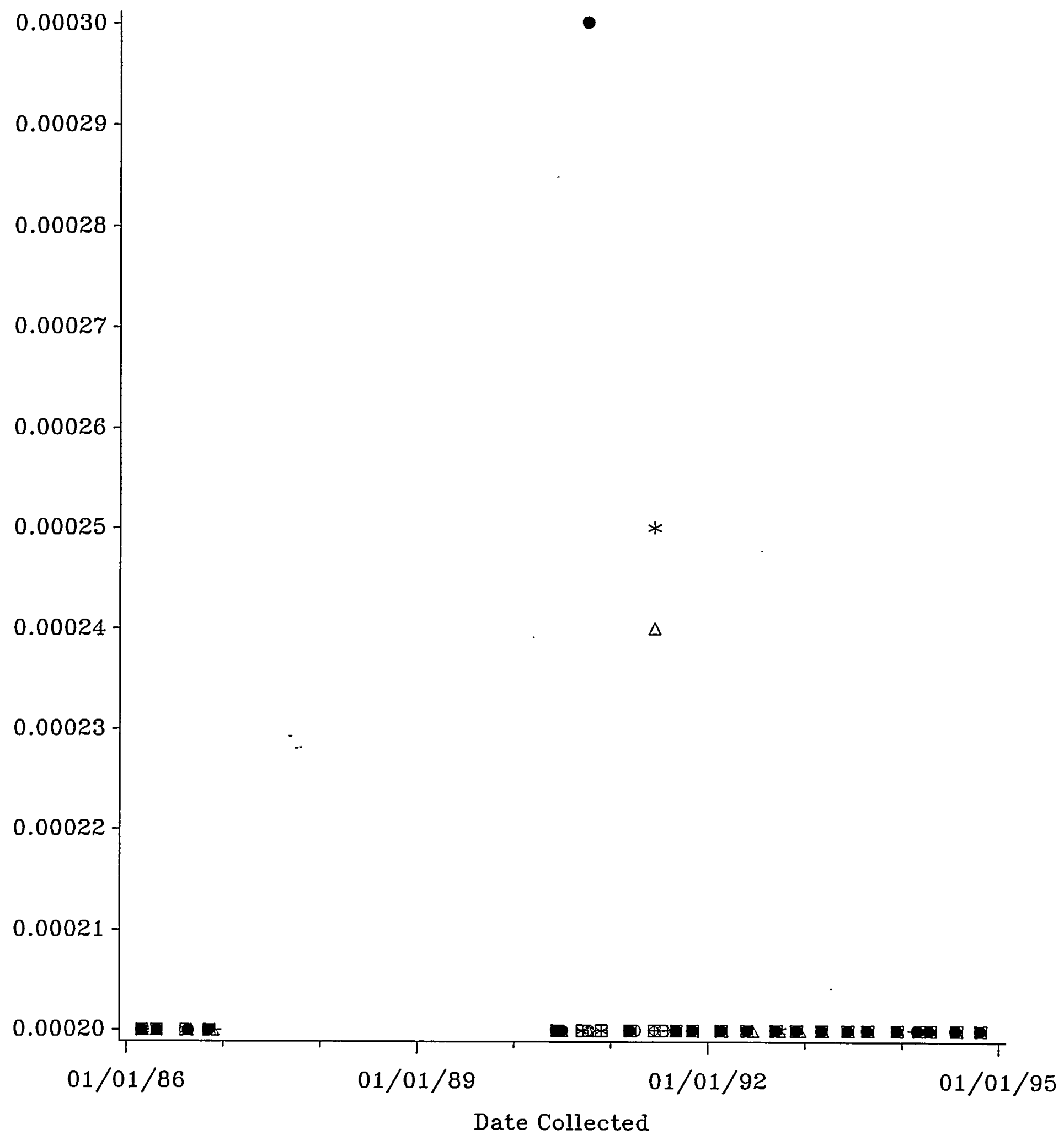

Well $+++\mathrm{GW}-142 * * * * \mathrm{GW}-143 \quad \square \square \square \mathrm{GW}-144 \quad \diamond \diamond \diamond \mathrm{GW}-145$ $\Delta \Delta \Delta \mathrm{GW}-146 \bullet \bullet \bullet \mathrm{GW}-147 \quad \circ \circ \circ \mathrm{GW}-231$

Figure F-6. Mercury concentrations over time.

nerated by program plot2 on $14 \mathrm{JUN} 95$ at 15:46 using datasets $\mathrm{y} 12 \mathrm{gw} \_\mathrm{mf}$ soilbg01 mndnew02 
Kerr Hollow Groundwater Data 1986-1994

Concentrations Over Time

Analyte (units) $=$ NICKEL $(M G / L)$

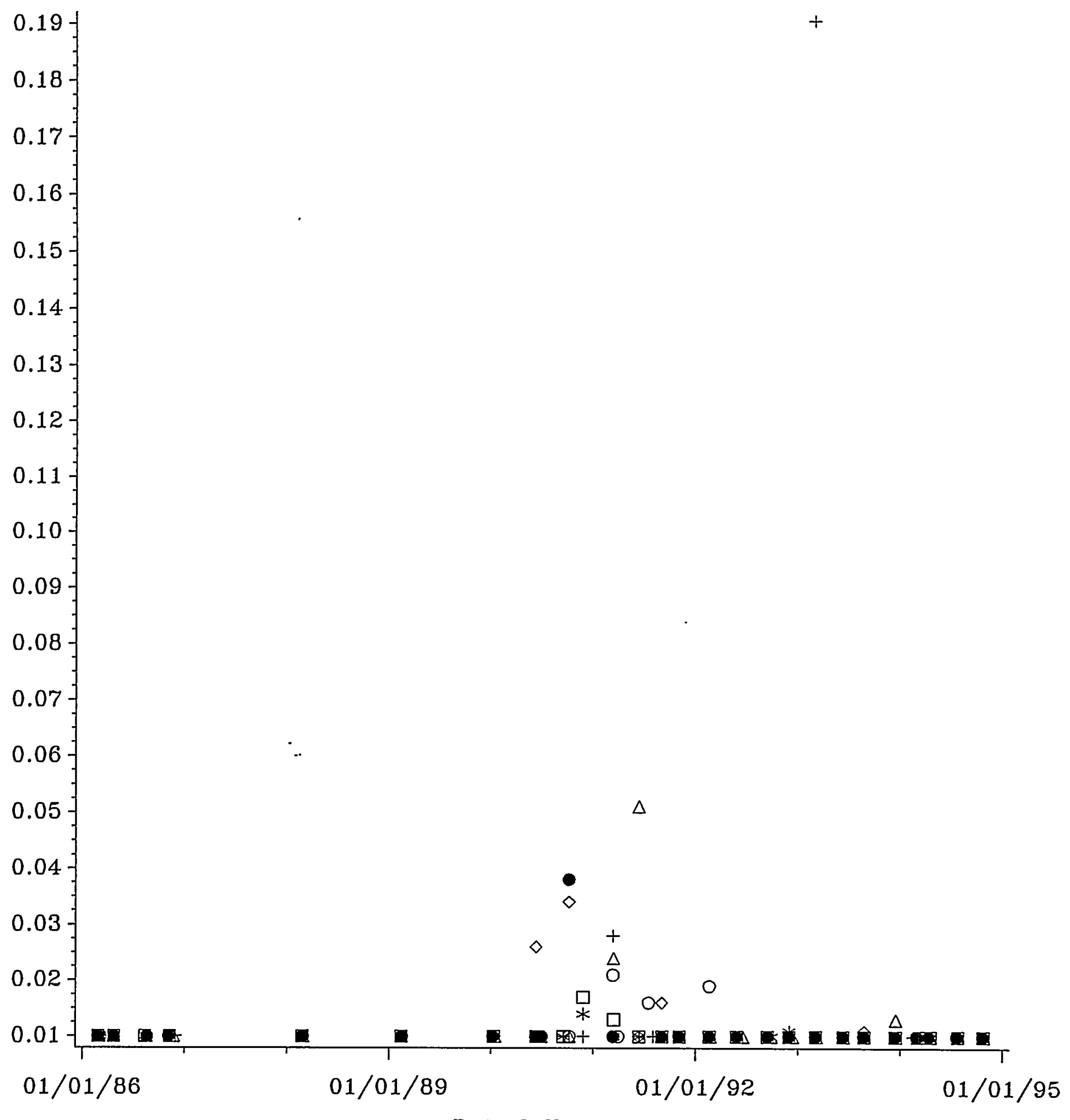

Date Collected

Well +++ GW-142 *** GW-143 ㅁ GW-144 $\diamond \diamond \diamond \mathrm{GW}-145$

$\Delta \Delta \Delta \mathrm{GW}-146 \quad \bullet \bullet \bullet \mathrm{GW}-147 \quad \circ \circ \circ \mathrm{GW}-231$

Figure F-7. Nickel concentrations over time.

, werated by program plot2 on 14JUN95 at 15:46 using datasets y12gw_mf soilbg01 mndnew02 
Kerr Hollow Groundwater Data 1986-1994

Concentrations Over Time

Analyte (units) $=$ STRONTIUM $(M G / L)$

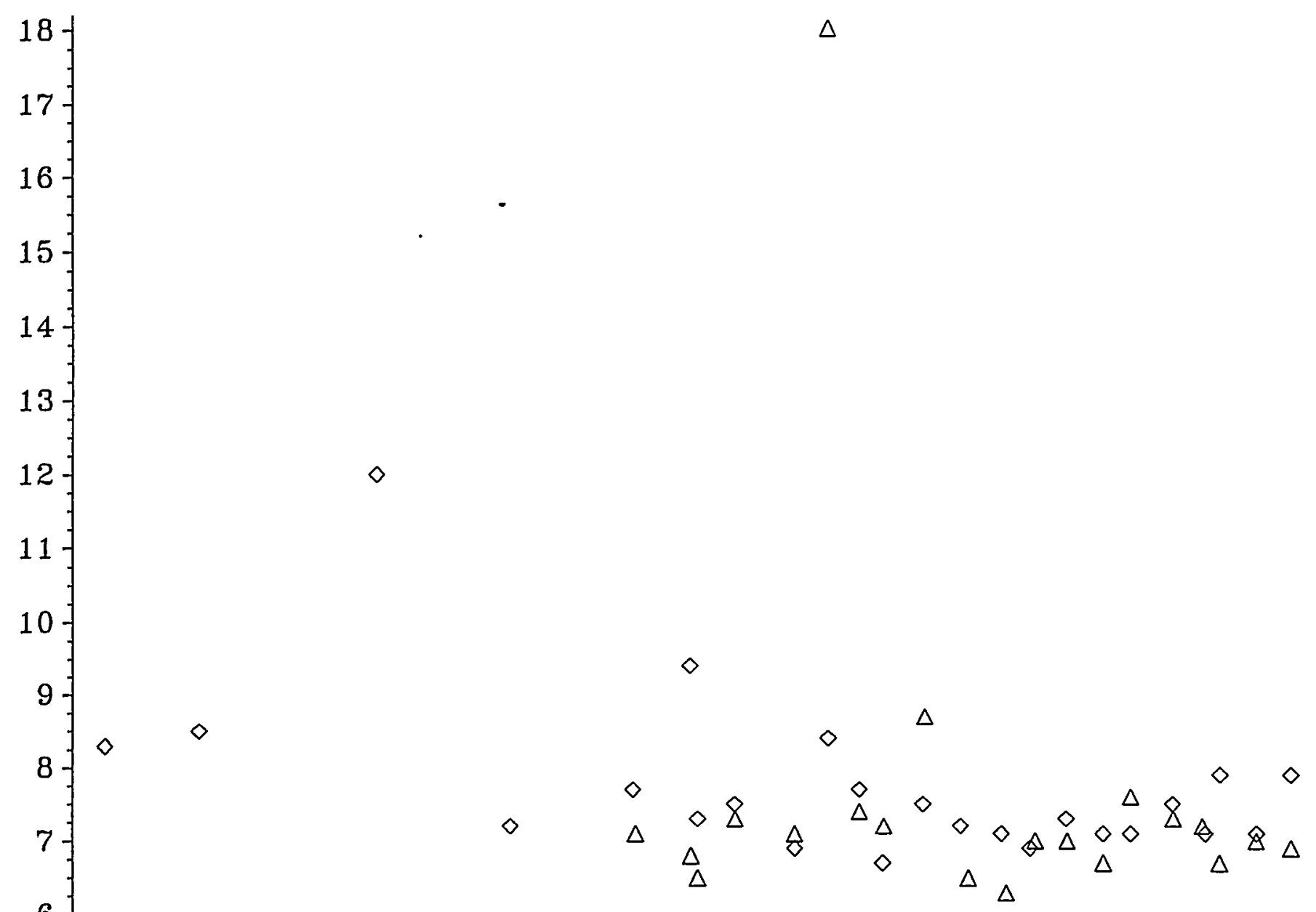

$\Delta$

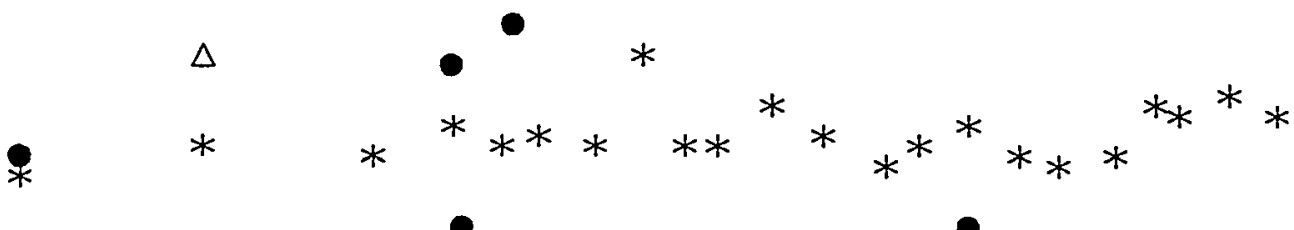

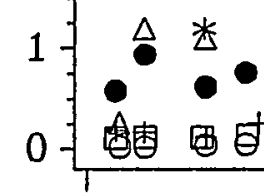

$01 / 01 / 86$

$01 / 01 / 89$

Date Collected

Well $+++\mathrm{GW}-142 * * * \mathrm{GW}-143 \quad \square \square \square \mathrm{GW}-144 \quad \diamond \diamond \diamond \mathrm{GW}-145$

$\Delta \Delta \Delta \mathrm{GW}-146 \quad \bullet \bullet \mathrm{GW}-147 \quad \circ \circ \circ \mathrm{GW}-231$

Figure F-8 Strontium concentrations over time.

nerated by program plot2 on $14 \mathrm{JUN} 95$ at $15: 46$ using datasets y12gw_mf soilbg01 mndnew02 
Kerr Hollow Groundwater Data 1986-1994

Concentrations Over Time

Analyte $($ units $)=$ TOTAL URANIUM (FLUOROMETRIC) $(\mathrm{MG} / \mathrm{L})$

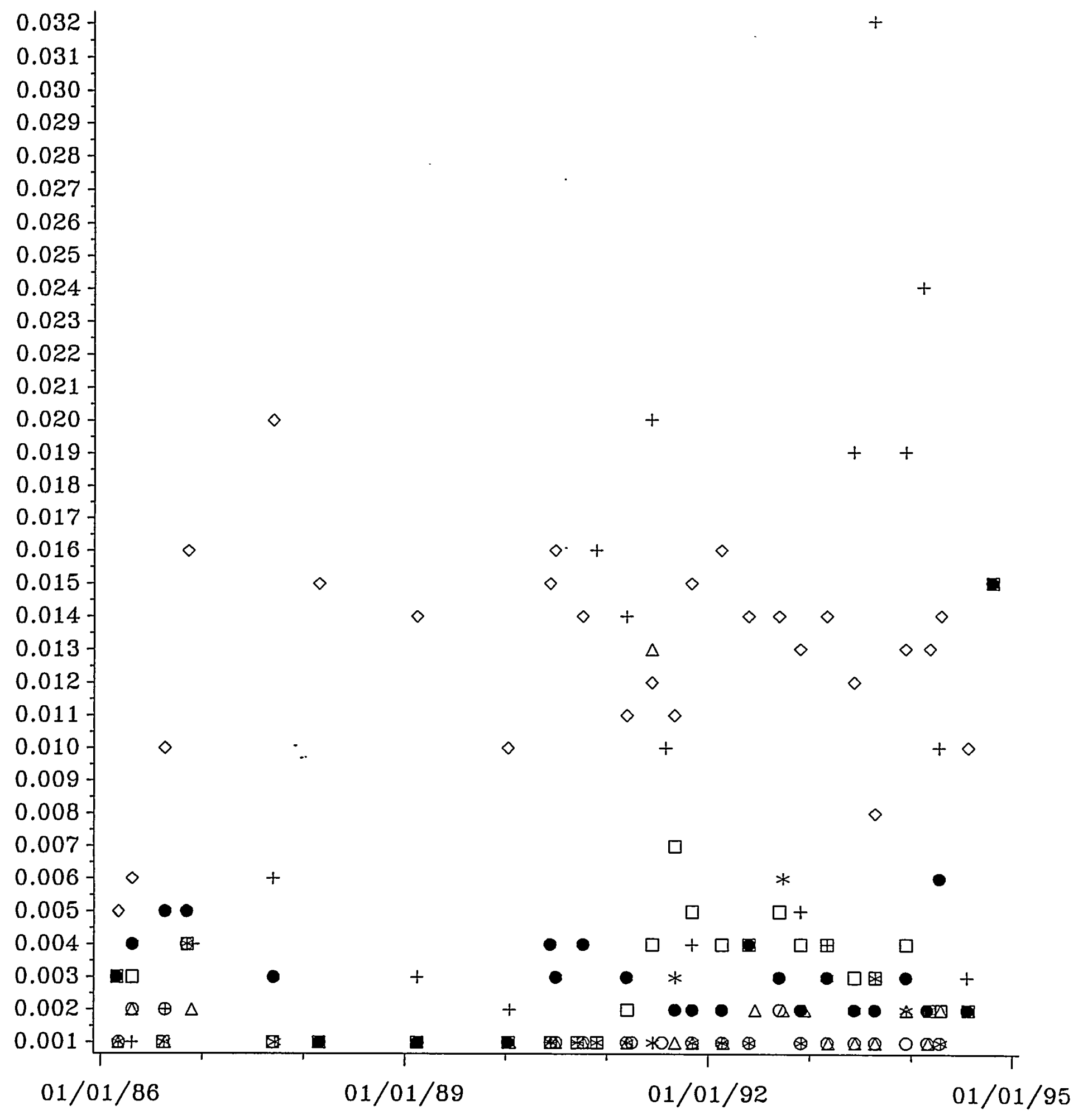

Date Collected

Well $+++\mathrm{GW}-142 * * * * \mathrm{GW}-143 \quad \square \quad \square \mathrm{GW}-144 \diamond \diamond \diamond \diamond \mathrm{GW}-145$

$\Delta \Delta \Delta \mathrm{GW}-146 \bullet \bullet-\mathrm{GW}-147 \quad \circ \circ \circ \mathrm{GW}-231$

Figure F-9. Total Uranium concentrations over time.

nerated by program plot2 on 14JUN95 at 15:46 using datasets y12gw_mf soilbg01 mndnew02 
Kerr Hollow Groundwater Data 1986-1994

Cumulative Frequency Plots

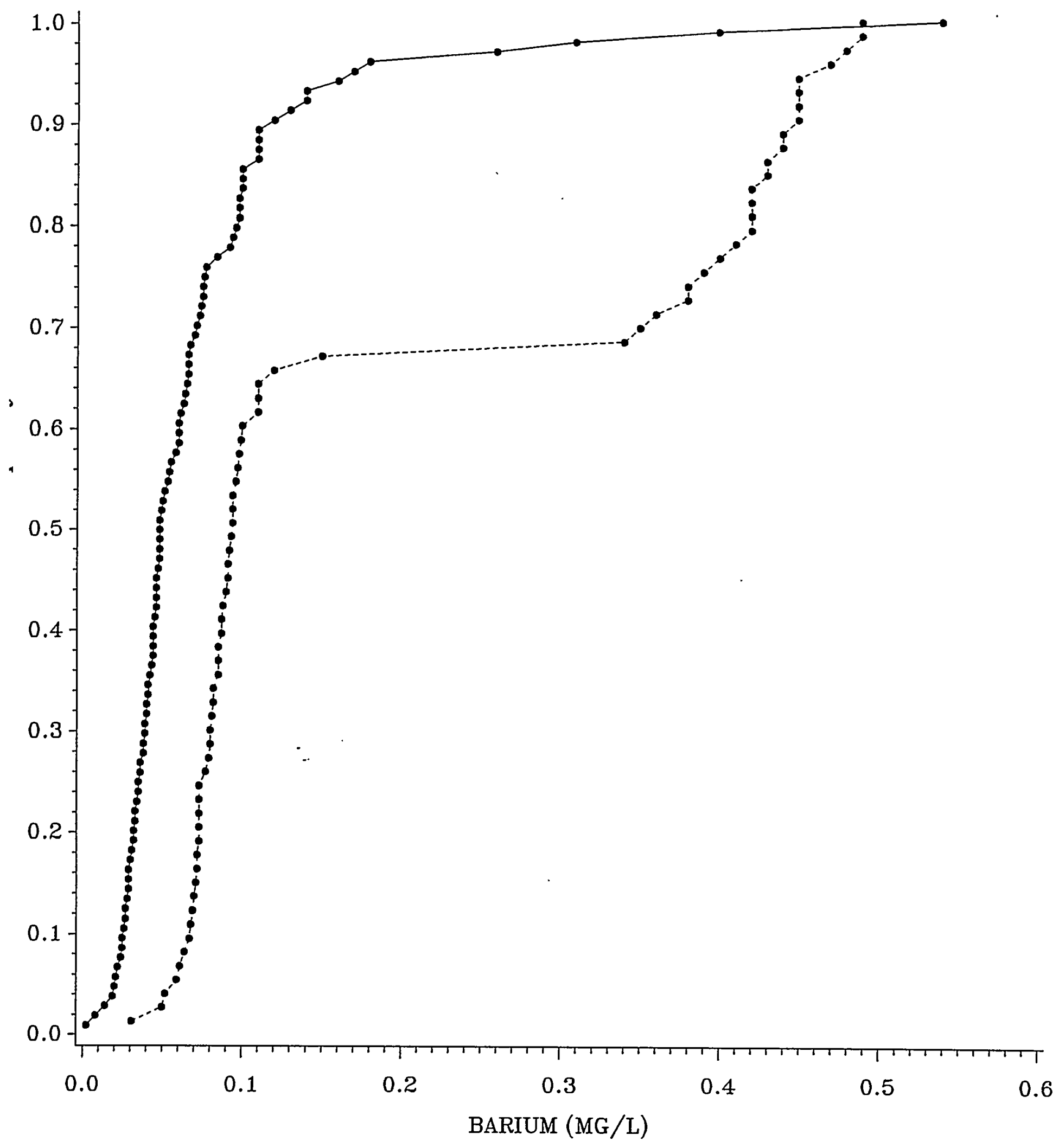

Area Down-Gradient --.---- Up-Gradient

Figure F-10. Barium cumulative frequency plots.

id symbols indicate detects. Open symbols represent nondetects. 
Kerr Hollow Groundwater Data 1986-1994

Cumulative Frequency Plots

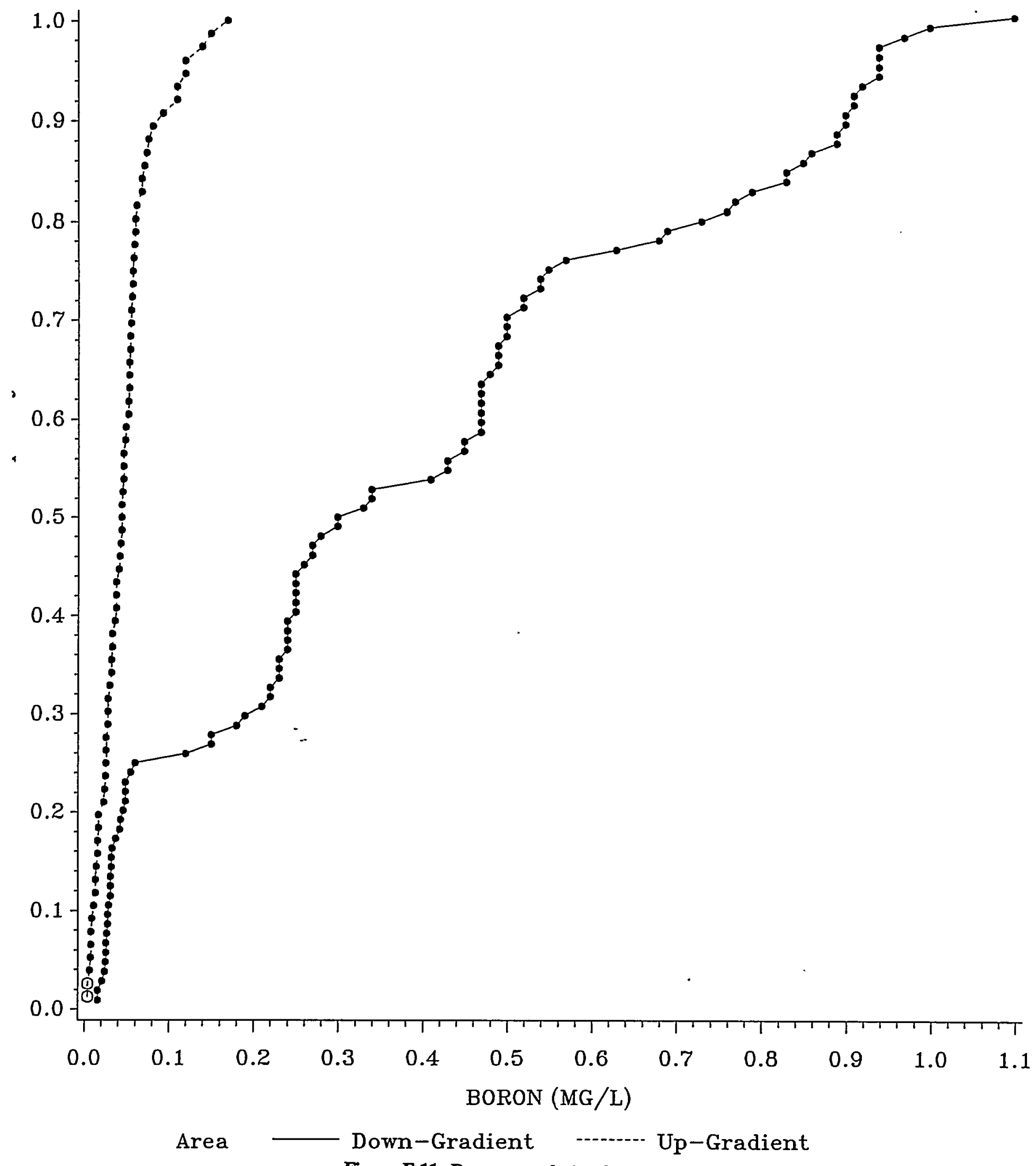

Figure F-11. Boron cumulative frequency plots.

id symbols indicate detects. Open symbols represent nondetects. 
Kerr Hollow Groundwater Data 1986-1994

Cumulative Frequency Plots

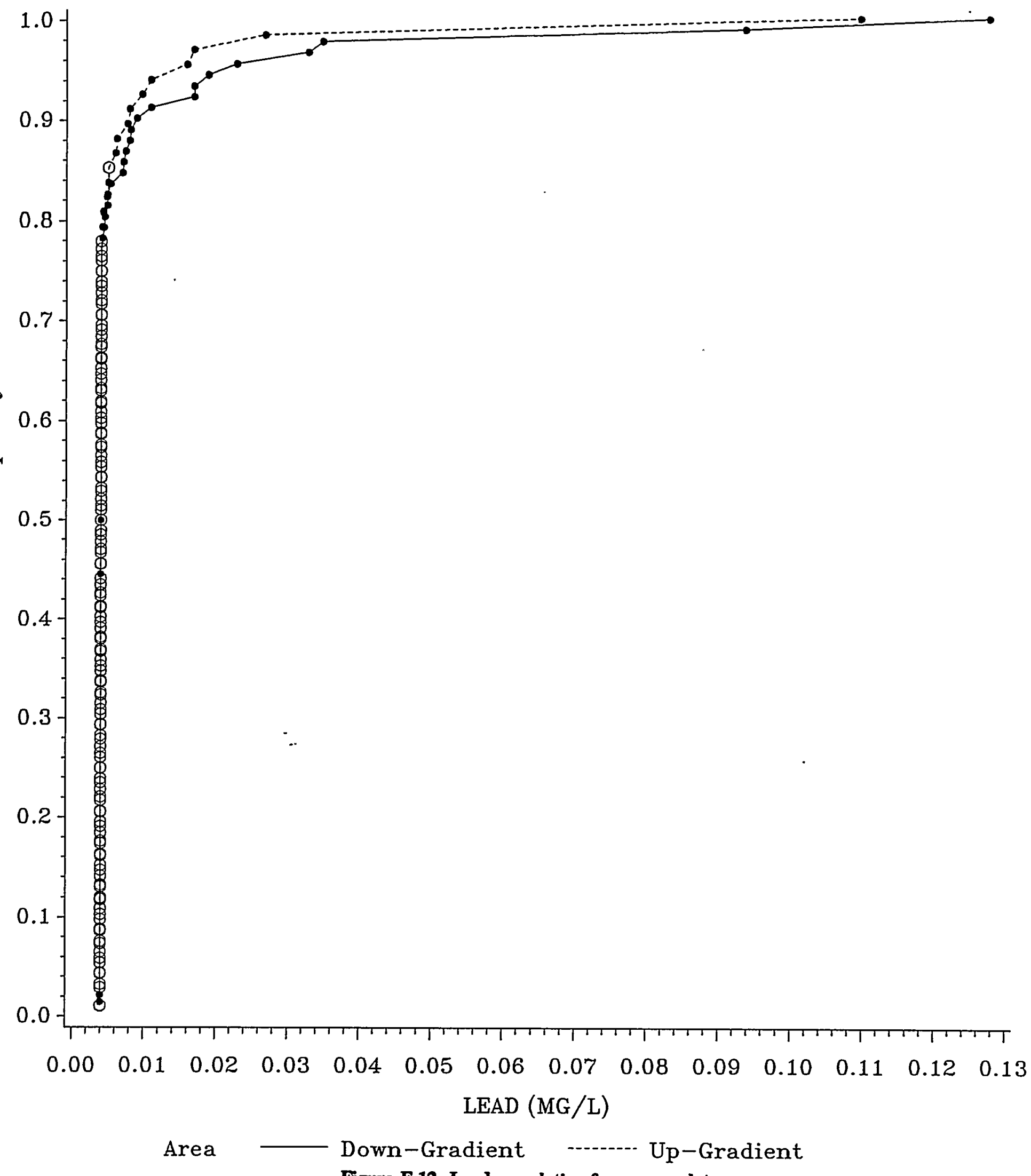

Figure F-12. Lead cumulative frequency plots.

lid symbols indicate detects. Open symbols represent nondetects. 
Kerr Hollow Groundwater Data 1986-1994

Cumulative Frequency Plots

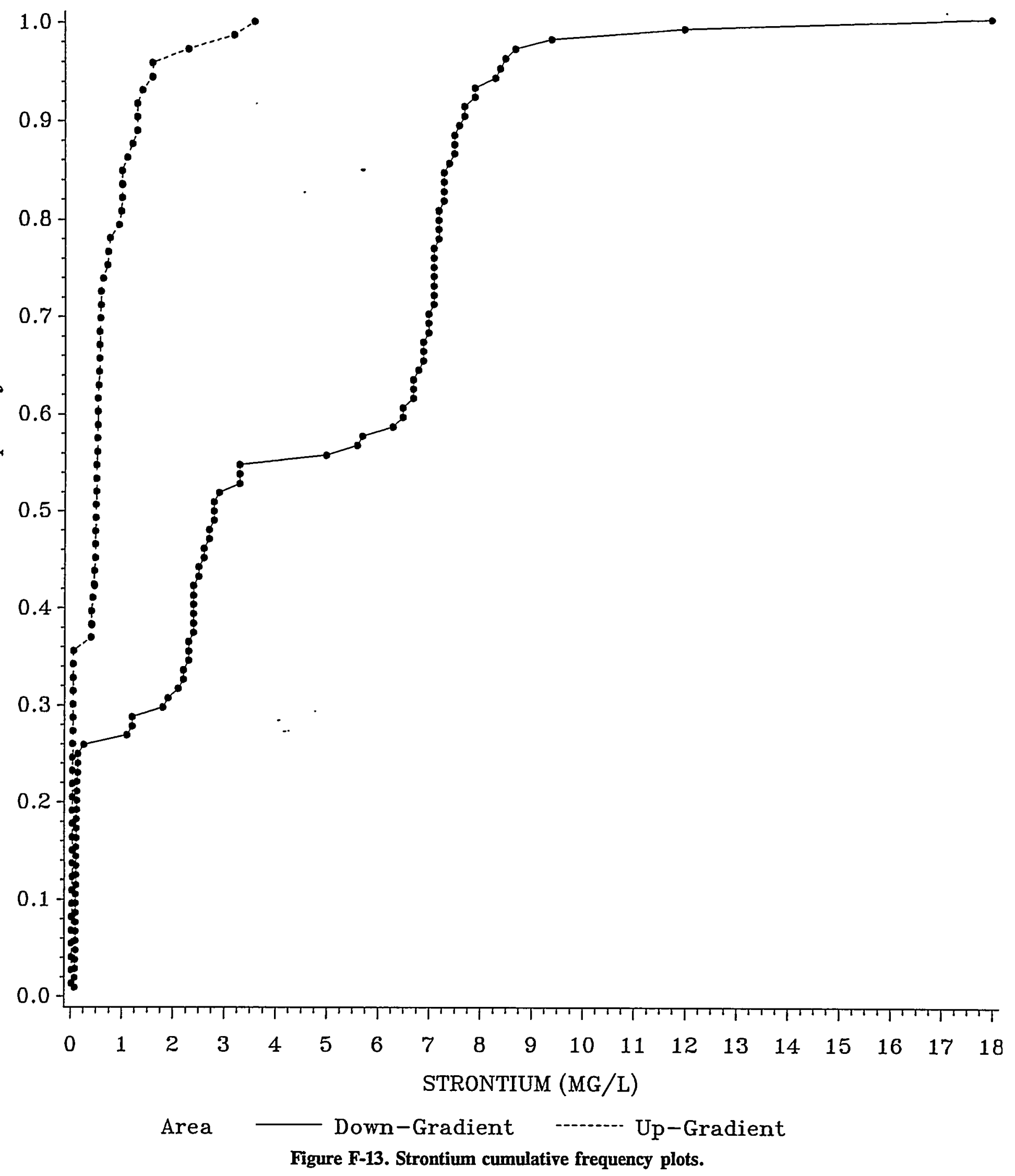

lid symbols indicate detects. Open symbols represent nondetects. 
Kerr Hollow Groundwater Data 1986-1994

Cumulative Frequency Plots

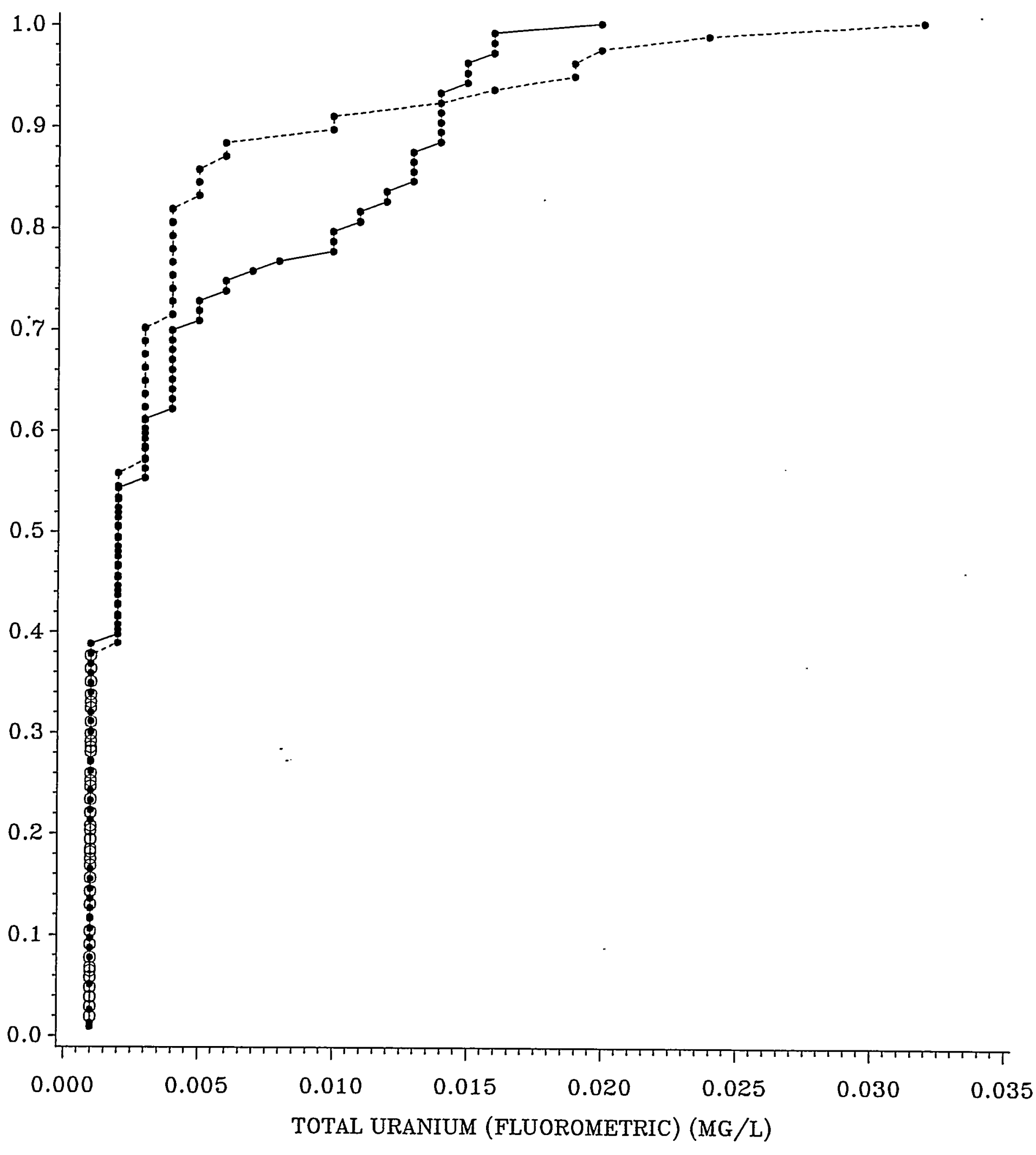

Area Down-Gradient ---.--- Up-Gradient

Figure F-14. Total Uranium cumulative frequency plots.

id symbols indicate detects. Open symbols represent nondetects. 